DANIELA VIEIRA CUNHA

\title{
ANÁLISE LÓGICA DE PROTOCOLOS, PROPOSTA E AVALIAÇÃO DE DESEMPENHO DE UM ALGORITMO DE ATRIBUIÇÃO DE RÓTULOS BASEADO EM SRLG EM UM AMBIENTE GMPLS-WDM
}

Tese apresentada a Escola Politécnica da Universidade de São Paulo para obtenção do título de Doutor em Engenharia. 
DANIELA VIEIRA CUNHA

\section{ANÁLISE LÓGICA DE PROTOCOLOS, PROPOSTA E AVALIAÇÃO DE DESEMPENHO DE UM ALGORITMO DE ATRIBUIÇÃO DE RÓTULOS BASEADO EM SRLG EM UM AMBIENTE GMPLS-WDM}

Tese apresentada a Escola Politécnica da Universidade de São Paulo para obtenção do título de Doutor em Engenharia.

Área de Concentração: Sistemas Digitais Orientadora: Prof. Dra Graça Bressan 


\section{FICHA CATALOGRÁFICA}

\section{Cunha, Daniela Vieira}

Análise lógica de protocolos, proposta e avaliação de desem-

penho de um algoritmo de atribuição de rótulos baseado em SRLG em um ambiente GMPLS-WDM / D.V. Cunha. -- São Paulo, 2006.

p.

Tese (Doutorado) - Escola Politécnica da Universidade de São Paulo. Departamento de Engenharia de Computação e Sistemas Digitais.

1.Protocolos de comunicação (Análise) 2.Redes ópticas I.Universidade de São Paulo. Escola Politécnica. Departamento de Engenharia de Computação e Sistemas Digitais Il.t. 


\section{FOLHA DE APROVAÇÃO}

Daniela Vieira Cunha

Análise Lógica de Protocolos, Proposta e Avaliação de Desempenho de um Algoritmo de Atribuição de Rótulos baseado em SRLG em um Ambiente GMPLS-WDM

Tese apresentada a Escola Politécnica da Universidade de São Paulo para obtenção do título de Doutor.

Área de Concentração: Sistemas Digitais

Aprovado em:

\section{Banca Examinadora}

Prof. Dr.

Instituição: Assinatura:

Prof. Dr.

Instituição: Assinatura:

Prof. Dr.

Instituição: Assinatura:

Prof. Dr.

Instituição: Assinatura:

Prof. Dr.

Instituição: Assinatura: 


\section{DEDICATÓRIA}

Aos meus queridos pais por todo amor e incentivo que sempre demonstraram.

Ao Rodrigo, meu marido, com amor, admiração e gratidão por sua compreensão, carinho, presença e incansável apoio ao longo do período de elaboração deste trabalho. 


\section{AGRADECIMENTOS}

A conclusão desta tese só foi possível devido a ajuda de várias pessoas e instituições. Então, eu gostaria de agradecer:

À Deus pela vida e por mais esta oportunidade.

À Fundação de Amparo à Pesquisa do Estado de São Paulo pela ajuda financeira recebida e pelas correções dos relatórios no decorrer do meu trabalho.

Ao Departamento de Engenharia da Computação e Sistemas Digitais (PCS) e Laboratório de Arquitetura e Redes de Computadores - LARC, na Escola Politécnica da Universidade de São Paulo, pelos recursos oferecidos para a execução deste trabalho.

À minha orientadora Prof. Dra. Graça Bressan, por sua orientação e dedicação na realização do curso de doutorado. Ela, que sempre me deu atenção, conselho e um melhor direcionamento nos momentos que precisei.

Aos funcionários e mais do que isso colegas do Laboratório, Ana Maria e Edivaldo, pela boa vontade e ajuda que sempre me ofereceram. Aos demais colegas do Laboratório que sempre me ensinaram e ajudaram a desenvolver meus conhecimentos.

Estes são presentes pra vida inteira. Mamãe e Papai contribuíram significativamente moral e espiritualmente para o desenvolvimento desta tese.

Ao meu marido Rodrigo que sempre esteve ao meu lado, e sempre me deu força, ânimo e exemplo de garra para continuar em frente mesmo nas horas difíceis.

Aos meus irmãos Leo e Vivinha, pelo carinho e apoio.

Aos meus avós, vovó Eliza, vovô Mauro, vovó Betinha e vovô Milton, que sempre se orgulharam de mim.

À todos os amigos e todas as pessoas que indiretamente me deram força e apoio. 


\section{RESUMO}

\section{Análise Lógica de Protocolos, Proposta e Avaliação de Desempenho de um Algoritmo de Atribuição de Rótulos baseado em SRLG em um Ambiente GMPLS-WDM}

Para satisfazer o explosivo aumento na demanda de tráfego de voz e dados, as redes ópticas baseadas em WDM e GMPLS estão sendo desenvolvidas. A suíte de protocolos GMPLS é atualmente considerada como um plano de controle para as redes ópticas e é composta por protocolos de sinalização e de roteamento, como também do protocolo de gerenciamento de enlace (LMP). O LMP é um importante protocolo que interfere na atribuição de rótulos (comprimentos de onda) e é necessário fazer sua análise lógica para verificar se o mesmo está livre de erros de progresso. Para esta finalidade, o método denominado alcançabilidade justa foi utilizado. Verificada a corretude do LMP, o estudo foca o subproblema de atribuição de comprimento de onda do RWA nas redes GMPLS-WDM por ser um dos principais problemas que causam o baixo desempenho destas redes. O cenário estudado é das redes GMPLS-WDM que operam em um ambiente RWA dinâmico com restrição de continuidade de comprimento de onda. O problema RWA é examinado bem como as várias heurísticas de atribuição de comprimento de onda apresentadas na literatura. Com o objetivo de melhorar o desempenho das redes GMPLS-WDM com restrição de continuidade de comprimento de onda, propõe-se um algoritmo de atribuição de rótulos que utiliza os conceitos conjunto de rótulos e SRLG já implementados pelo GMPLS. O algoritmo proposto melhora a eficiência no uso de recursos nas redes em questão. O desempenho é verificado através da métricas de probabilidade de bloqueio de conexão, desempenho este próximo do ótimo e demonstrado através de simulações.

Palavras-Chave - GMPLS, redes ópticas, análise lógica, alcançabilidade justa, RWA, algoritmo de atribuição de rótulos, conjunto de rótulos, SRLG. 


\begin{abstract}
Protocol Logical Analysis, Proposal and Performance Evaluation of a Label Assignment Algorithm based on SRLG in a GMPLS-WDM Environment
\end{abstract}

To satisfy the explosive increasing demands of voice and data traffic, optical networks based on WDM and GMPLS are being developed. The GMPLS' suite of protocols is currently being considered as the control plane for optical networks and it is compounded of signaling and routing protocols, and also the link management protocol (LMP). The LMP is an important protocol that interferes with label (wavelength) assignment and it is necessary to logically analyse this protocol in order to verify if it is free from progress errors. For this purpose, the method called fair reachability has been used. Verified the LMP is correctable, the study focuses on the RWA wavelength assignment problem in GMPLS-WDM networks because it is one of the main problems which causes the low performance of these networks. The studied scene is GMPLS-WDM networks operating under a dynamic RWA environment with wavelength continuity constraint. The RWA problem is examined and also the various wavelength-assignment heuristics proposed in the literature. With the goal to improve the performance of the GMPLS-WDM networks with wavelength continuity constraint, it is proposed a label assignment algorithm, which uses the concepts of label set and SRLG, already implemented by GMPLS. The proposed algorithm provides an improvement in efficiency of resource use. The performance is verified by using the blocking probability metric, and it is very close to the optimum and demonstrated through simulations.

Key-words - GMPLS, optical networks, logical analysis, fair reachability, RWA, label assignment protocol, label set, SRLG. 


\section{SUMÁRIO}

\section{CAPÍTULO I - INTRODUÇÃO}

1.1 GMPLS

1.2 Redes GMPLS Ópticas Baseadas em WDM 021

1.3 SRLG

1.4 Objetivo e Justificativa

1.5 Metodologia

1.6 Organização do Texto

\section{CAPÍTULO II - GMPLS - MPLS GENERALIZADO}

2.1 Introdução

2.2 Evolução em Direção ao GMPLS

2.2.1 MPLS e o Paradigma Label Switching

2.2.2 MPLS-TE

2.2.3 MP $\lambda \mathrm{S}$

2.2.4 Conceitos Fundamentais do GMPLS

2.3 Rótulos Generalizados

2.3.1 Pedido de Rótulo Generalizado

2.3.2 Escolha de Rótulos com Restrição

2.4 Interfaces Suportadas

2.5 Conjunto de Protocolos

2.5.1 Protocolos de Roteamento

2.5.2 Protocolos de Sinalização

2.5.3 Protocolo de Gerenciamento de Enlace

2.6.1 Descobrimento de Vizinhos

2.6.2 Cálculo de Caminhos

2.6.3 Sinalização de Serviço

2.7 Grupos de Enlaces de Risco Compartilhado - SRLG 


\section{CAPÍTULO III - REDES GMPLS BASEADAS EM WDM - VISÃO GERAL DO}

RWA

3.1 Introdução

3.2 Redes Ópticas WDM

3.3 Roteador de Comprimento de Onda

3.4 Encaminhamento de Tráfego

3.4.1 Sem Conversão de Comprimento de Onda

3.4.2 Com Conversão de Comprimento de Onda

3.4.3 Com Conversão x Sem Conversão

3.5 Roteamento e Atribuição de Comprimento de Onda

3.5.1 Subproblema de Roteamento

3.5.2 Subproblema de Atribuição de Comprimento de Onda

3.5.3 Formulação Matemática - atribuição de comprimento de onda

\section{CAPÍTULO IV - ANÁLISE LÓGICA DE PROTOCOLOS DE COMUNICAÇÃO 101}

4.1 Introdução

4.2 Estratégias de Reparação Existentes

4.3 Redes de Máquinas de Estado Finitas de Comunicação

\section{CAPÍTULO V - ANÁLISE LÓGICA, PROPOSTA DE CORREÇÕES E MELHORIAS DO PROTOCOLO DE GERENCIAMENTO DE ENLACE}

5.1 Introdução

5.2 LMP - Visão Geral

5.3 Gerenciamento de Canal de Controle

5.3.1 Negociação de Parâmetros

5.3.2 Protocolo Hello

5.3.3 Canal de Controle Desativado

5.3.4 Estado Degradado 
5.5 Verificação de Conectividade de Enlace

5.6 Gerenciamento de Falhas

5.7 Procedimentos de Back-off Exponencial

5.8 Máquinas de Estado Finitas

5.8.1 MEF de Canal de Controle

5.8.2 MEF de Enlace TE

5.8.3 MEF de Enlace de Dados

5.8.4 MEF de Gerenciamento de Falhas

\section{CAPÍTULO VI - ATRIBUIÇÃO DE RÖTULOS COM BASE NA INFORMAÇÃO} SRLG EM UM AMBIENTE GMPLS-WDM 164

$\begin{array}{ll}6.1 \text { Introdução } & 164\end{array}$

$\begin{array}{ll}6.2 \text { Proposta } & 166\end{array}$

6.2.1 Algoritmo Proposto 167

$\begin{array}{ll}\text { 6.2.2 Análise Lógica } & 170\end{array}$

$\begin{array}{ll}\text { 6.3 Simulação } & 177\end{array}$

6.3.1 Descrição dos Simuladores 178

6.3.2 Topologias Simuladas, Avaliação de Desempenho,

Apresentação e Análise dos Resultados 179

6.3.2.1 Topologia de 6 Nós 181

6.3.2.2 Topologia NSFNET 189

6.3.2.3 Topologia Mesh 195

6.3.2.4 Topologia EON 201

6.3.3 Validação do Simulador 206

$\begin{array}{ll}6.4 \text { Escalabilidade } & 207\end{array}$

$\begin{array}{ll}6.5 \text { Conclusão } & 211\end{array}$

CAPÍTULO VII - CONCLUSÃO $\quad 214$

REFERÊNCIAS $\quad 222$ 


\section{LISTA DE FIGURAS}

Figura 2.1 - Comparação entre o roteamento IP convencional (topo) e o Paradigma Label Switching (base)

Figura 2.2 - Hierarquia de Envio

Figura 2.3 - Alocação de Rótulos para LSPs Bidirecionais com Estabelecimento Simultâneo

Figura 2.4 - Rótulos Generalizados

Figura 2.5 - Conflito na Escolha de Rótulos com Restrição em Comutadores Ópticos

Figura 2.6 - Como estabelecer um caminho óptico com sinalização RSVP-TE

Figura 2.7 - Interfaces suportadas pelo GMPLS

Figura 2.8 - Fluxo de Informação e Controle GMPLS

Figura 2.9 - Rede GMPLS

Figura 2.10 - SRLG - Exemplo de um modelo de rede

Figura 3.1 - Rede com roteamento de comprimento de onda

Figura 3.2 - Roteador de comprimento de onda

Figura 3.3 - Conversor de comprimento de onda

Figura 3.4 - Encaminhamento de tráfego

Figura 3.5 - Anel óptico unidirecional com 6 nós e 4 comprimentos de onda

Figura 4.1 - Representação de um Protocolo Simples através de uma Máquina de

Estado

Figura 4.2 - (a) e (b) especificação de dois processos que formam um protocolo através de máquinas de estado finitas; (c) análise de alcançabilidade exaustiva do protocolo; (d) análise de alcançabilidade justa do protocolo especificado 
Figura 5.2 - Rede de MEFCs de canal de controle formada a partir da MEF de canal de controle

Figura 5.3 - Grafo de Alcançabilidade Justa resultante da rede de MEFCs do LMP relacionado ao canal de controle

Figura 5.4 - MEF de enlace TE

Figura 5.5 - Rede de MEFCs de enlace TE formada a partir da MEF de enlace TE

Figura 5.6 - Grafo de Alcançabilidade Justa resultante da rede de MEFCs do LMP relacionado ao enlace TE

Figura 5.7 - MEF de Enlace de Dados Ativo

Figura 5.8 - MEF de Enlace de Dados Passivo

Figura 5.9 - Rede de MEFCs de enlace de dados (ativo e passivo) formada a partir das MEFs de enlaces de dados ativo e passivo

Figura 5.10 - Grafo de Alcançabilidade Justa resultante da rede de MEFCs do LMP relacionado ao enlace de dados passivo

Figura 5.11 - Rede de MEFCs de gerenciamento de falhas

Figura 5.12 - Grafo de Alcançabilidade Justa resultante da rede de MEFCs do LMP relacionado ao gerenciamento de falhas

Figura 6.1 - Definição do protocolo de sinalização para atribuição de comprimento de onda

Figura 6.2 - Máquina de estado finito de comunicação entre nó upstream e um nó downstream em um ponto intermediário da rede

Figura 6.3 - Análise justa da MEFC da sinalização de atribuição de rótulos em um ponto intermediário da rede

Figura 6.4 - Máquina de estado finito de comunicação entre nó upstream e um nó downstream considerado nó de egresso da rede 
Figura 6.5 - Análise justa da MEFC da sinalização de atribuição de rótulos no ponto de egresso da rede

Figura 6.6 - Topologia com 6 nós

Figura 6.7 - Topologia de 6 nós com M=1 e W=16 comprimentos de onda sem problema de Traps

Figura 6.8 - Topologia de 6 nós com $\mathrm{M}=2$ e $\mathrm{W}=8$ comprimentos de onda sem problema de Traps

Figura 6.9 - Topologia de 6 nós com $\mathrm{M}=4$ e $\mathrm{W}=4$ comprimentos de onda sem problema de Traps

Figura 6.10 - Topologia de 6 nós com M=1 e W=16 comprimentos de onda com problema de Traps

Figura 6.11 - Topologia de 6 nós com $\mathrm{M}=2$ e $\mathrm{W}=8$ comprimentos de onda com problema de Traps

Figura 6.12 - Topologia de 6 nós com M=4 e W=4 comprimentos de onda com problema de Traps

Figura 6.13 - Topologia NSFNET

Figura 6.14 - Topologia NSFNET com 32 comprimentos de onda sem problema de Traps

Figura 6.15 - Topologia NSFNET com 40 comprimentos de onda sem problema de Traps

Figura 6.16 - Topologia NSFNET com 32 comprimentos de onda com problema de Traps

Figura 6.17 - Topologia NSFNET com 40 comprimentos de onda com problema de Traps 
Figura 6.18 - Topologia Mesh com 25 nós

Figura 6.19 - Topologia Mesh com 32 comprimentos de onda sem problema de Traps

Figura 6.20 - Topologia Mesh com 40 comprimentos de onda sem problema de Traps

Figura 6.21 - Topologia Mesh com 32 comprimentos de onda com problema de Traps

Figura 6.22 - Topologia Mesh com 40 comprimentos de onda com problema de Traps

Figura 6.23 - Topologia EON 202

Figura 6.24 - Topologia EON com 32 comprimentos de onda sem problema de Traps

Figura 6.25 - Topologia EON com 40 comprimentos de onda sem problema de Traps

Figura 6.26 - Topologia EON com 32 comprimentos de onda com problema de Traps

Figura 6.27 - Topologia EON com 40 comprimentos de onda com problema de Traps

Figura 6.28 - Tempo de Atribuição de Comprimento de Onda na Topologia de 6 nós 210 Figura 6.29 - Tempo de Atribuição de Comprimento de Onda na Topologia NSFNET 210 


\section{LISTA DE TABELAS}

Tabela 3.1 - Possíveis conexões em uma rede em anel unidirecional com 6 nós

Tabela $3.2-\mathrm{Pb}_{\text {prox }}$ após o estabelecimento da conexão $(2,4)$

Tabela 3.3 - Perda relativa por comprimento de onda

096

Tabela 6.1 - Esboço da tabela SRLG_ $\lambda$ 


\section{LISTA DE SIGLAS}

\begin{tabular}{|c|c|}
\hline $\mathrm{ADM}$ & Add/Drop Multiplexer \\
\hline AP & Available Pool \\
\hline ASPR & Alternative Shortest Path Routing \\
\hline ATM & Asynchronous Transfer Mode \\
\hline BGP & Border Gateway Protocol \\
\hline BoD & Bandwidth on Demand \\
\hline BW & Bandwidth \\
\hline CAPEX & Capital Expenditures \\
\hline $\mathrm{CC}$ & Canal de Controle \\
\hline CCAMP & Common Control and Measurement Information \\
\hline CC-ID & Control Channel Identifier \\
\hline CFSMs & Communicating Finite State Machines \\
\hline $\mathrm{CoS}$ & Class of Service \\
\hline CR-LDP & Constraint-based Routing - Label Distribution Protocol \\
\hline CSPF & Constraint-based Shortest Path First \\
\hline DLE & Dynamic Lightpath Establishment \\
\hline DRCL & Distributed Relative Capacity Loss \\
\hline DWDM & Dense Wavelength Division Multiplexing \\
\hline $\mathrm{DXC}$ & Digital Crossconnect \\
\hline $\mathrm{ECN}$ & Explicit Congestion Notification \\
\hline EF & Explicited Forwarding \\
\hline EGP & Exterior Gateway Protocol \\
\hline
\end{tabular}


Enlaces Ópticos

EON

European Optical Network

ERO

Explicit Route Object

FA

Forwarding Adjacency

FEC

Forwarding Equivalence Class

FF

First Fit

FIB

Forwarding Information Base

FIFO

First In First Out

FR

Frame Relay

FRG

Fair Reachability Graph

FSC

Fiber Switch Capable

FSM

Finite State Machine

GAJ

Grafo de Alcançabilidade Justa

G-LSP

Generalized Label Switched Path

GMPLS

Generalized Multiprotocol Label Switching

G-PID

Generalized Payload Identifier

ID

Identifier

IETF

Internet Engineering Task Force

IGP

Interior Gateway Protocol

IP

Internet Protocol

IS-IS

Intermdiate System - Intermediate System

IS-IS TE

Intermediate System - Intermediate System Traffic Engineering

ISP

Internet Service Provider

LDP

Label Distribution Protocol

LER

Label Edge Router 


\begin{tabular}{|c|c|}
\hline LLR & Least loaded routing \\
\hline LMP & Link Management Protocol \\
\hline LoL & Loss of Light \\
\hline LR & Label Request \\
\hline LSA & Link State Advertisement \\
\hline LSC & Lambda Switch Capable \\
\hline LSP & Label Switched Path \\
\hline LSR & Label Switching Router \\
\hline LU & Least Used \\
\hline MEFC & Máquinas de Estado Finitas de Comunicação \\
\hline MEMS & Micro Electro Mechanical Systems \\
\hline $\mathrm{MP} \lambda \mathrm{S}$ & Multiprotocol Lambda Switching \\
\hline MPLS & Multiprotocol Label Switching \\
\hline MS & Max Sum \\
\hline MU & Most Used \\
\hline MUX & Multiplexing \\
\hline NAM & Network Animator \\
\hline NMS & Network Management System \\
\hline $\mathrm{NS} 2$ & Network Simulator - Versão 2 \\
\hline NSFNET & National Science Foundation Network \\
\hline OADM & Optical Add-Drop Multiplexors \\
\hline Och & Optical Channel \\
\hline OEO & Optical - Electrical - Optical \\
\hline OIF & Optical Internetworking Forum \\
\hline OO & Optical - Optical \\
\hline
\end{tabular}




\begin{tabular}{|c|c|}
\hline OPEX & Operating Expenditures \\
\hline OSC & Optical Switch Controller \\
\hline OSPF & Open Shortest Path First \\
\hline OSPF-TE & Open Shortest Path First - Traffic Engineering \\
\hline OVPN & Optical Virtual Private Network \\
\hline OWNS & Optical WDM Network Simulator \\
\hline $\mathrm{OXC}$ & Optical Cross-connect \\
\hline PSC & Packet Switch Capable \\
\hline QoS & Quality of Service \\
\hline RCL & Relative Capacity Loss \\
\hline $\mathrm{RD}$ & Random Wavelength Assignment \\
\hline RFC & Request for Comments \\
\hline ROI & Return on Investment \\
\hline RSVP & Resource Reservation Protocol \\
\hline RSVP-TE & Resource Reservation Protocol - Traffic Engineering \\
\hline RWA & Routing and Wavelength Assignment \\
\hline SDH & Synchronous Digital Hierarchy \\
\hline SLA & Service Level Agreement \\
\hline SLE & Static Lightpath Establishment \\
\hline SNMP & Simple Network Management Protocol \\
\hline SP & Service Provider \\
\hline SPF & Shortest Path First \\
\hline SONET & Synchronous Optical Network \\
\hline SPR & Shortest Path Routing \\
\hline SRLG & Share Risk Link Group \\
\hline
\end{tabular}


SRLG-ID

TCP

TDM

TE

ToS

TSC

UDP

UP

VPN

WDM

$\mathrm{XC}$
Identificador (número) de SRLG

Transmission Control Protocol

Time Division Multiplex

Traffic Engineering

Type of Service

TDM Switch Capable

User Datagram Protocol

Used Pool

Virtual Private Network

Wavelength Division Multiplexing

Cross-connect 


\section{CAPÍTULO I}

\section{INTRODUÇÃO}

Desde que a rede de comunicação de dados baseada na arquitetura TCP/IP (Transmission Control Protocol / Internet Protocol), a Internet, foi aberta ao tráfego comercial, esta cresceu rapidamente de uma rede de pesquisa experimental, restrita a um campus, para uma rede de comunicação de dados pública abrangente e de grande escala. A demanda faz com que a capacidade da Internet hoje cresça em várias dimensões mensuráveis de uma rede incluindo, mas não se limitando à largura de banda na transmissão, número de hosts, qualidade de serviço (QoS - Quality of Service), tamanho geográfico e volume de tráfego. Ao mesmo tempo, a Internet está evoluindo do serviço baseado no melhor esforço convencional (best effort), passando a oferecer serviços com níveis de garantia de QoS e incorporando novas tecnologias à medida que se desenvolve [CUNHA, 2001].

Existem aplicações que apresentam serviços de entrega críticos com relação ao tempo e qualidade. Juntamente com a crescente importância desses serviços de dados, há também uma mudança nos tipos de aplicações disponíveis. As tradicionais aplicações (e.g. e-mail, ftp) 
estão cada vez mais incorporando características de aplicações em tempo real, tais como serviços de voz e de vídeo. As características de tempo real destas novas aplicações demandam por QoS. Logo, é necessário que as redes de comunicação suportem contratos de nível de serviço (SLA - Service Level Agreement) ${ }^{1}$ que garantem um nível específico de QoS, independente do serviço utilizado como também de falhas na rede.

Há alguns anos, a quantidade de tráfego de dados sendo encaminhada em todas as redes no mundo já ultrapassou a quantidade de tráfego de voz e essa diferença tende a continuar aumentando nos próximos anos, tornando crescente os requisitos de largura de banda para dados IP e atingindo limites que demandam a reconstrução da arquitetura de rede e mudanças na infra-estrutura de transporte existente. Ao mesmo tempo, tem-se uma grande e crescente demanda dos usuários para que se mantenham baixos os custos dos serviços oferecidos pela rede.

A necessidade de transportar mais dados, combinada com a necessidade de minimizar o custo de transporte desses dados, resulta em uma situação onde Provedores de Serviço (SPs Service Providers) carecem de soluções que permitem o encaminhamento de um grande volume de tráfego de forma mais eficiente e a um custo menor; o que foi considerado um desafio caso fosse utilizada a arquitetura de rede de dados em uso atualmente.

Acredita-se, indústria e alguns pesquisadores, que a rede óptica é a melhor solução capaz de suportar todos os requisitos de serviço e de qualidade resultantes desse crescimento, em volume de tráfego, tamanho geográfico e diversificação de aplicações. Desta forma, substancial número de pesquisas tem como foco as redes ópticas cujo desenvolvimento provê

\footnotetext{
${ }^{1}$ SLA: é um contrato entre um provedor de serviço de rede e um cliente que especifica, geralmente em termos mensuráveis, quais os serviços que o provedor de serviço de rede deve fornecer e com qual qualidade.
} 
aumento da capacidade de transporte e da escalabilidade da rede e, o desenvolvimento de uma grande quantidade de novas e sofisticadas aplicações [PAPADIMITRIOU, 2003].

Para que isto aconteça, requer-se uma maior integração da camada IP com a camada óptica, como também uma maior padronização dos protocolos nos ambientes de comutação de telecomunicações de hoje. No entanto, para que potenciais benefícios da convergência IP e redes ópticas possam ser utilizados, serviços IP em particular precisam ser mais inteligentes, flexíveis e escaláveis, objetivo que pode ser atingido através do uso da tecnologia MPLS (Multiprotocol Label Switching), e possíveis extensões, nas redes IP.

Para se ter uma rede verdadeiramente dinâmica, é necessário um método para controle total da rede dentro do núcleo óptico. Surgiu então, o conceito de rede óptica inteligente. O MPLS poderia ser utilizado, mas como é específico para redes IP, seus protocolos são modificados para "conversar" com equipamentos de telecomunicação, propiciando desta forma o surgimento do MPLS Generalizado (GMPLS - Generalized MPLS).

\subsection{GMPLS}

O MPLS foi definido apenas para redes de comutação de pacotes e as pesquisas sobre esta tecnologia estão mais direcionadas para o plano de dados (tráfego de dados). Por outro lado, o GMPLS estende a arquitetura MPLS permitindo o interfaceamento com tecnologias que não são apenas baseadas na comutação de pacotes. O GMPLS está sendo desenvolvido com o objetivo de ser um conjunto de protocolos aplicáveis a todo tipo de tráfego de transporte e serviço. Ele suporta não somente comutação de pacotes (PSC - Packet Switching Capable),

mas também comutação no domínio do tempo (TSC - TDM, Time Division Multiplex, 
Switching Capable), comprimento de onda (LSC - Lambda Switching Capable) e fibra (FSC Fiber Switching Capable) [IOVANNA, 2003], [IEC], [DEO, 2003].

Com suporte do IETF (Internet Engineering Task Force) e do OIF (Optical Internetworking Forum), o GMPLS está rapidamente tornando-se um padrão. O IETF foca na estrutura GMPLS como um todo e, o OIF desenvolve especificações de interoperabilidade destes protocolos [ROUSSEAU, 2003].

O GMPLS, extensão do paradigma MPLS às redes ópticas, é um conjunto de protocolos do plano de controle que fornece uma semântica consistente e uniforme para sinalização (RSVPTE - Resource Reservation Protocol - Traffic Engineering), roteamento (OSPF-TE - Open Shortest Path First - Traffic Engineering) e gerenciamento de enlace (LMP - Link Management Protocol) [LANDOLSI, 2002], [PAPADIMITRIOU, 2003], [ROUSSEAU, 2003].

\subsection{REDES GMPLS ÓPTICAS BASEADAS EM WDM}

Com o objetivo de melhorar o aproveitamento da capacidade de transmissão das fibras ópticas, as redes ópticas, incluindo as redes GMPLS, passaram a usar o WDM (Wavelength Division Multiplexing), uma técnica de multiplexação que divide a fibra óptica em vários canais WDM de diferentes freqüências ópticas, chamados de comprimentos de onda [ASSI, 2001], [CHAUDHURI, 2000], [KIM, 2003].

Através de um caminho óptico (G-LSP - label switched path generalizado, ou seja, caminho generalizado baseado em comutação de rótulos) uma conexão em uma rede óptica é 
estabelecida utilizando um determinado comprimento de onda. Em geral, seria desejável poder alocar um comprimento de onda diferente para cada pedido de conexão, porém, nas redes ópticas WDM, o número de comprimentos de onda disponíveis nos enlaces limita o número possível de conexões entre cada par de nós [ZHU, 2000], [CHAUDHURI, 2000], [STRAND, 2001].

Dentro das redes ópticas, o tráfego em cada conexão estabelecida pode ser encaminhado pela rede de duas formas:

- Sem conversão de comprimento de onda

Um caminho óptico usa o mesmo comprimento de onda em todos os enlaces ópticos que o compõem, da origem até o destino. Essa propriedade é chamada de restrição de continuidade de comprimento de onda. Duas conexões que compartilham um enlace comum devem utilizar comprimentos de onda distintos [ZANG, 2000], [ROUSKAS, 2001].

- Com conversão de comprimento de onda

Diferentes comprimentos de onda podem ser usados pelos enlaces das rotas de conexões ao longo do caminho óptico, ou seja, pode haver conversão de um comprimento de onda para outro comprimento de onda em um mesmo caminho óptico. Neste tipo de encaminhamento, é necessária a utilização de conversores nos roteadores para converter o comprimento de onda nos nós intermediários [ZANG, 2000], [ROUSKAS, 2001].

Porém a tecnologia de conversores de comprimento de onda ainda não está amadurecida e competitiva e, os mesmos possuem um custo muito alto. Deste modo, o foco deste estudo é 
em redes GMPLS ópticas que não possuem a capacidade de conversão de comprimento de onda.

Um problema muito importante nas redes GMPLS ópticas baseadas em WDM é a configuração de caminhos ópticos para um dado conjunto de pedidos de conexões através de roteamento e atribuição de comprimentos de onda (rótulos ${ }^{2}$ ) para cada uma dessas conexões. Este problema é denominado de problema de roteamento e atribuição de comprimento de onda - RWA (Routing and Wavelength Assignment) [ZHU, 2000], [ASSI, 2001], [CHAUDHURI, 2000], [STRAND, 2001], [ZANG, 2000], [OKI, 2004].

O objetivo do RWA é selecionar a melhor combinação de rotas e comprimentos de onda para cada conexão de forma a otimizar o atendimento das demandas dentro da rede, maximizando o número de conexões estabelecidas e/ou minimizando o número de comprimentos de onda usados pela rede, assim melhorando a eficiência da mesma.

O subproblema de roteamento se restringe em encontrar um caminho para um par origemdestino de um pedido de conexão. O problema é atualmente tratado com as seguintes técnicas:

- Roteamento fixo [GIRARD, 1990]

- Roteamento fixo alternativo [GIRARD, 1990], [HARAI, 1997], [RAMAMURTHY, 1998]

- Roteamento adaptativo [JUE, 2000], [LI, 1999]

No subproblema de atribuição de comprimento de onda, deve-se alocar um comprimento de onda para cada caminho óptico de tal forma que dois caminhos ópticos não compartilhem um

\footnotetext{
${ }^{2}$ No caso das redes GMPLS ópticas, um rótulo específico é sinônimo de um comprimento de onda particular.
} 
mesmo comprimento de onda em uma mesma fibra. Os principais algoritmos de atribuição de comprimento de onda propostos na literatura são:

- $\quad$ First-Fit [CHLAMTAC, 1989]

- Random Wavelength Assignment [CHLAMTAC, 1989]

- Most-Used [SUBRAMANIAM, 1997]

- Least-Used [SUBRAMANIAM, 1997]

- Max-Sum [BARRY, 1997], [SUBRAMANIAM, 1997]

- Relative Capacity Loss [ZHANG, 1998]

- Distributed Relative Capacity Loss [ZANG, 2000]

Quando há demanda em uma rede GMPLS totalmente óptica (i.e., GMPLS-WDM) e é preciso realizar roteamento e seleção de comprimento de onda, um pedido de conexão pode falhar devido a um dos dois eventos de bloqueio denominados bloqueio de enlace forward e bloqueio de enlace backward. O bloqueio de enlace forward é devido a recursos de comprimento de onda insuficientes e a não utilização de algoritmos de roteamento que promovem o balanceamento no uso dos enlaces. Bloqueio de enlace backward, ou simplesmente bloqueio de conexão ${ }^{3}$, acontece devido ao conflito de reservas de rótulos [JERRAN, 2001].

Com o objetivo de diminuir o bloqueio de conexão em um ambiente GMPLS-WDM com restrição de continuidade de comprimento de onda, o GMPLS introduz o conceito de conjunto de rótulos (no inglês, Label Set) para restringir a escolha do rótulo pelo nó downstream ${ }^{4}$ para um pedido de caminho óptico [BERGER, 2003], [OKI, 2004].

\footnotetext{
${ }^{3} \mathrm{O}$ termo bloqueio de conexão será utilizado no restante deste trabalho.

${ }^{4}$ Nó downstream: próximo nó vizinho com relação ao fluxo de dados.
} 
O objeto conjunto de rótulos não é suficiente para eliminar o bloqueio de conexão que ocorre quando dois ou mais nós de egresso selecionam o mesmo rótulo para conexões que compartilham os mesmos enlaces.

\subsection{SRLG}

SRLG (Shared Risk Link Group, no português, grupo de enlaces de risco compartilhado), fornece parâmetros fundamentais para o gerenciamento de recursos no plano de controle GMPLS e necessários para planejar a confiabilidade nas redes de transporte. Um SRLG está associado com uma entidade em risco, tipicamente um segmento de fibra. Assim, um SRLG é definido como um conjunto de enlaces que compartilham um risco em comum [PAPADIMITRIOU - Draft, 2003].

O conceito SRLG foi concebido no desenvolvimento de mecanismos de recuperação de falhas. Ele (SRLG) é considerado como um dos critérios mais importantes com relação a comutação de caminhos baseados em restrições nas rotas de canal óptico. Aplicando o critério de disjunção SRLG na computação de caminhos baseados em restrições, pode-se selecionar uma rota levando em consideração a separação de estrutura lógica e de recursos. Isto implica em uma probabilidade mais baixa de falhas de canal óptico simultaneamente [SEBOS, 2002], [STRAND, 2001], [OKI, 2004], [MIYAMURA, 2004].

\subsection{OBJETIVO e JUSTIFICATIVA}

O GMPLS ainda não se tornou um padrão por completo e nem mesmo uma RFC (Request for Comments). Alguma documentação desta tecnologia se encontra apenas como Internet Drafts. Antes mesmo de padronizar o MPLS Generalizado, é de grande importância fazer uma análise lógica dos protocolos que compõem o conjunto de protocolos GMPLS. Neste trabalho, esta 
análise é feita utilizando a técnica de alcançabilidade com progresso justo podendo assim verificar o comportamento do protocolo LMP (protocolo de gerenciamento de enlace) do conjunto de protocolos GMPLS bem como verificar se o mesmo está ou não livre de erros de progresso. Os demais protocolos que compõem o GMPLS são os protocolos de roteamento e sinalização que são os mesmos utilizados anteriormente pelo MPLS e possuem apenas algumas extensões para suportar diferentes interfaces de comutação no MPLS Generalizado. Trabalhos anteriores já verificaram a corretude desses protocolos [IETF] e por está razão essas análises não fazem parte do escopo deste trabalho.

Após a análise lógica do protocolo LMP, é possível propor uma adequação à atribuição de rótulos em um ambiente GMPLS com o intuito atender ao problema RWA.

Com o objetivo de melhorar o desempenho e a utilização de recursos de uma rede GMPLSWDM com restrição de continuidade de comprimento de onda, diminuindo a probabilidade de bloqueio de conexão, propõe-se um novo algoritmo de atribuição de comprimento de onda (rótulos) que utiliza o conceito de conjunto de rótulos juntamente com o conceito SRLG que passa a ser utilizado no processo de atribuição de comprimento de onda e não somente no estabelecimento dos caminhos de proteção.

A idéia para alocação de comprimentos de onda é que para conexões cujos caminhos ópticos compartilham pelo menos um enlace na rede, deve-se atribuir diferentes comprimentos de onda para cada conexão, pois a propriedade de restrição de continuidade de comprimento de onda é considerada. Esta é a mesma idéia proposta pelo conceito SRLG para o estabelecimento de caminhos de proteção e que agora será usado para alocação de comprimento de onda, como proposta deste trabalho. Com base na informação SRLG do 
caminho a ser estabelecido e dos caminhos já existentes é possível saber se estes compartilham ou não alguma entidade de risco (recurso) e assim decidir qual comprimento de onda deve ser atribuído.

\subsection{METODOLOGIA}

Para que haja um ordenado desenvolvimento do estudo proposto, alguns procedimentos devem ser realizados tais como: levantamento bibliográfico relevante aos assuntos abordados, estudo aprofundado dos temas relacionados ao trabalho tais como ferramentas de avaliação lógica e experiência no manuseio de simulador.

$\mathrm{Na}$ etapa inicial é realizado um levantamento bibliográfico, análise e estudo do material selecionado sobre o ambiente GMPLS e os protocolos de roteamento e sinalização utilizados, bem como o protocolo de gerenciamento de enlace, LMP. Este levantamento bibliográfico é feito através de revistas especializadas, artigos já publicados e RFCs na forma de Internet Drafts (devido ao fato do GMPLS ser uma tecnologia nova e ainda estar em desenvolvimento e padronização). As principais referências utilizadas foram: [ROUSSEAU, 2003], [LANDOLSI, 2002], [PAPADIMITRIOU, 2003], [IOVANNA, 2003], [IEC], [DEO, 2003], [CHOI, 2003], [LANG, 2005].

Posteriormente, um estudo mais detalhado é efetuado sobre o problema de roteamento e atribuição de comprimento de onda (RWA) em um ambiente GMPLS como também os conceitos e uso do SRLG. O problema de roteamento e atribuição de comprimento de onda existe em redes com conversão de comprimento de onda e sem conversão de comprimento de onda, e vantagens e desvantagens são encontradas em cada um destes cenários. A forma de estabelecimento de caminhos ópticos em um ambiente GMPLS-WDM considera o uso do 
conjunto de rótulos na tentativa de diminuir a probabilidade de bloqueio de conexão e assim atender ao problema RWA. As mais significativas referências utilizadas são: [ASSI, 2001], [CHAUDHURI, 2000], [KIM, 2003], [ZHU, 2000], [CHAUDHURI, 2000], [STRAND, 2001], [ZANG, 2000], [ROUSKAS, 2001], [OKI, 2004].

Com base nos estudos realizados, verifica-se que o protocolo LMP ainda não foi analisado de acordo com sua corretude e necessário é antes de propor qualquer alteração no controle GMPLS para atribuição de rótulos atendendo assim o problema RWA. O protocolo de gerenciamento de enlace pode ser então modelado por uma rede formada por máquinas de estado finitas de comunicação (MEFC).

MEFCs são largamente utilizadas para especificar e validar protocolos de comunicação através da análise de alcançabilidade. Este método provou ser um dos métodos mais efetivos na verificação da corretude dos protocolos de comunicação, no entanto, sabe-se também que a explosão de estados é a mais severa limitação da aplicabilidade deste método [SCHOOT, 1998], [GOUDA, 1984], [MOURA, 1986], [LIN, 1998], [NEGULESCU, 1995].

$\mathrm{Na}$ busca por uma abordagem apropriada para lidar com tal problema, são pesquisadas diferentes estratégias de reparação desta limitação. As abordagens estudadas são:

- Estratégia de reparação proposta por West que restringe o uso de parâmetros multivalorados, limita o número de mensagens nos canais de mensagens e limita as classes de erros de transmissão [WEST, 1982]

- Estratégias de reparação propostas por Vuong e Cowan, Choi e Miller e, Chow, Gouda e Lam. Eles observaram que certas classes de protocolos podem ser particionadas em componentes que posteriormente podem ser separadamente verificados para garantir a corretude do protocolo original. [VUONG, 1982], [CHOI, 1983], [CHOW, 1985] 
- Estratégia proposta por Lam e Shankar. Uma abordagem de projeção que constrói um protocolo imagem do protocolo dado para cada uma das funções que se pretende verificar [LAM, 1984]

- Estratégia proposta por Blumer e Sidhu. Utiliza um mecanismo chamado regra de escolha de transição que é uma condição booleana cujo valor decide se a transição na MEFC é ou não para ser executada [BLUMER, 1986]

- Estratégia proposta por Ansart. Faz uso de uma ferramenta denominada LISE baseada no modelo de máquina de estado finita estendida [ANSART, 1985]

- Estratégias propostas por Ruben e West, Gouda e Han e, Zhao e Bochman. Tem como base a exploração de estado com progresso justo. A idéia é explorar somente os estados globais que são alcançáveis considerando o fato das duas entidades de protocolo avançarem em uma mesma velocidade [RUBIN, 1982], [GOUDA, 1985], [ZHAO, 1987]

- Estratégia proposta por Gouda e Yu. Proposta chamada de exploração de estado com progresso máximo [GOUDA, 1984]

- Estratégia proposta por Itoh e Ichikawa. Proposta aplicada a protocolos cuja MEFC não contém qualquer ciclo que não passe pelos estados iniciais [ITOH, 1983]

- Estratégia proposta por Brand e Zfiropulo e, Kakuda, Wakahara e Morigoe. Estratégia chamada de validação de protocolo de árvore ou forma acíclica [BRAND, 1983], [KAKUDA, 1986]

- Holzmann projetou uma ferramenta chamada Trace, que trabalha sob dois modos, como ferramenta de depuração rápida ou como um provador de corretude lento [HOLZMANN, 1985] [HOLZMANN, 1987] [HOLZMANN, 1988] 
- Estratégia proposta por West. Esta estratégia é chamada de exploração de estados com caminhada aleatória (random-walk) que é uma forma de parcialmente explorar o grafo de estados globais. [WEST, 1987]

- Estratégia proposta por Vuong, Hui, e Cowan. Os pesquisadores propõem uma nova representação de estado global de forma que o algoritmo de alcançabilidade desenvolvido gere grafos finitos para todos os protocolos não-FIFO e para uma certa classe de protocolos FIFO [VUONG, 1982].

Dentre as abordagens apresentadas a exploração de estados com progresso justo foi a abordagem escolhida para a análise do protocolo LMP devido às suas vantajosas características.

Alcançabilidade justa [GOUDA, 1986] é uma técnica muito útil de detecção de erros de progresso tais como deadlocks, recepções não-especificadas, interações não-executadas, livelocks e overflow em redes de MEFCs compostas por duas máquinas [PENG, 1997].

Finalizada a avaliação do protocolo LMP, apresenta-se a proposta de uma nova técnica de atribuição de comprimento de onda com o objetivo de diminuir a probabilidade de bloqueio de conexão em ambientes GMPLS-WDM com restrição de continuidade de comprimento de onda e assim melhorar a eficiência e desempenho dessas redes. A proposta se baseia no conceito de conjunto de rótulos já utilizado em ambientes GMPLS e no conceito SRLG usado em mecanismos de proteção. O objetivo é utilizar conceitos já implementados com sucesso que ao serem utilizados em abordagens diferentes, podem ajudar a atender o problema RWA. 
A proposta é também analisada de acordo com sua corretude, ou seja, se está livre ou não dos erros de progresso e, a técnica de alcançabilidade justa é usada para esta finalidade. A análise realizada é da porção do protocolo de sinalização relacionada com atribuição de rótulos.

A análise lógica não é suficiente para comprovar a eficiência ou não eficiência da proposta de atribuição de rótulos. É necessário realizar a avaliação de desempenho da mesma, o que é uma tarefa complexa e trabalhosa. Em geral, três soluções podem ser utilizadas para auxiliar na execução desta tarefa: a experimentação com redes reais, a utilização de métodos analíticos e a simulação computacional. Cada uma dessas soluções possui vantagens e limitações e, embora não sejam mutuamente excludentes, cada uma delas é melhor aplicável a uma determinada situação particular. Este trabalho utiliza a simulação computacional para realizar a análise da proposta de atribuição de rótulos. Assim, é necessário o uso de um simulador para que o mesmo comprove ou não a queda na taxa de probabilidade de bloqueio de conexão para diferentes cenários GMPLS-WDM.

Apresenta-se uma avaliação de desempenho dos algoritmos de atribuição de comprimento de onda citados anteriormente bem como do algoritmo proposto com relação a probabilidade de bloqueio de conexão. Assim, é possível verificar o desempenho oferecido pelo algoritmo de atribuição de comprimento onda baseado em SRLG.

O programa de simulação Network Simulator - NS versão 2.1b6, juntamente com o módulo OWNS (Optical WDM Network Simulator), bem como o Matlab foram utilizados para que a avaliação relacionada a cada heurística de atribuição de comprimento de onda mencionada e proposta fosse obtida. A base do simulador foi mantida e apenas algumas modificações e 
acréscimos foram feitos de forma a adaptar o simulador a cada um dos algoritmos de atribuição de comprimento de onda.

\subsection{ORGANIZAÇÃO DO TEXTO}

A organização deste trabalho foi realizada como descrito a seguir.

Capítulo II apresenta uma visão geral do GMPLS, suas principais características e funcionamento, a evolução desde o MPLS bem como os protocolos de sinalização, roteamento e gerenciamento que nessa tecnologia são utilizados. A forma de estabelecimento de caminhos ópticos em um ambiente GMPLS-WDM é descrita como também o uso do conjunto de rótulos na tentativa de diminuir a probabilidade de bloqueio de conexão. Para finalizar o Capítulo, algumas definições do conceito SRLG e sua importância são apresentadas.

Capítulo III aborda as redes ópticas WDM, o problema de roteamento e atribuição de comprimento de onda (RWA) em redes ópticas com conversão de comprimento de onda e sem conversão de comprimento de onda. Vantagens e desvantagens são discutidas para cada um dos casos mencionados. São apresentadas as técnicas de roteamento utilizadas no subproblema de roteamento bem como as heurísticas de atribuição de comprimento de onda propostas pela literatura e utilizadas no subproblema de atribuição de comprimento de onda.

Capítulo IV descreve a forma como protocolos de comunicação podem ser avaliados de acordo com sua corretude. São apresentados alguns tipos de erros de progresso e também as diferentes técnicas existentes para a finalidade de avaliação. Foco maior é dado à análise de alcançabilidade justa por ter sido a técnica escolhida para aplicação neste trabalho. 
Capítulo V relata a análise lógica do protocolo de gerenciamento de enlace (LMP) que faz parte do conjunto de protocolos GMPLS. A técnica de alcançabilidade justa é aplicada às máquinas de estado finitas de comunicação que representam cada função deste protocolo. Com base nessa análise verifica-se a corretude ou não corretude do LMP.

Capítulo VI apresenta a proposta de um novo algoritmo de atribuição de comprimento de onda utilizando o conceito de conjunto de rótulos e o conceito SRLG em um ambiente GMPLS-WDM com o objetivo de diminuir a probabilidade de bloqueio de conexão. É detalhada a análise lógica desta proposta através da técnica de alcançabilidade justa. Descreve-se os aspectos relevantes à simulação de uma rede GMPLS-WDM para os diversos algoritmos de atribuição de comprimento de onda, a apresentação dos resultados das simulações e uma breve análise destes, possibilitando assim a comparação entre as heurísticas de atribuição de comprimento de onda mencionadas e a proposta.

As conclusões gerais e algumas sugestões para continuidade deste trabalho são mencionadas no Capítulo VII. 


\section{CAPÍTULO II}

\section{GMPLS - MPLS GENERALIZADO}

\subsection{INTRODUÇÃO}

O desenvolvimento óptico iniciado no início dos anos 90 rapidamente conduziu a um impressionante interesse em inúmeros tópicos de pesquisa tais como engenharia de tráfego (traffic engineering - TE), recuperação (proteção e restauração), controle in-band e out-ofband e, técnicas de supervisão e monitoramento. No domínio de transmissão, as principais atividades foram direcionadas não somente para a comutação por comprimento de onda, mas também para o DWDM (Dense Wavelength Division Multiplexing), que é uma tecnologia que aumenta o número de comprimentos de ondas em uma única fibra.

O tráfego de Internet, incluindo serviços relacionados, tem crescido significativamente desde 1995, bem como sua importância tem aumentado de forma contínua. Hoje, é amplamente 
reconhecido que o tráfego IP (Internet Protocol) continuará crescendo ${ }^{5}$ e constituirá cada vez mais uma maior proporção do volume total de tráfego na rede ${ }^{6}$. Estas tendências criam uma necessidade para uma eficiente interoperação entre redes orientadas a circuito tradicionais e redes IP/MPLS (Internet Protocol / Multiprotocol Label Switching) baseadas no paradigma de encaminhamento/comutação de pacotes.

Atualmente, a mais promissora tecnologia capaz de atender os rígidos requisitos das operadoras (aumento no rendimento ou diminuição de despesas operacionais) é o conjunto de protocolos GMPLS (MPLS Generalizado, no inglês Generalized Multiprotocol Label Switching) como definido pelo IETF (Internet Engineering Task Force) e pelo OIF (Optical Internetworking Forum).

Os esforços de padronização começaram no final dos anos 90 com o desenvolvimento do MPגS (Multiprotocol Lambda Switching) que focou somente nas redes ópticas de próxima geração. Desde então, MP $\lambda S$ foi aprimorado gerando o GMPLS, definido como um conjunto de protocolos centrada no IP para planos de controle [ROUSSEAU, 2003].

GMPLS é uma generalização da arquitetura MPLS [RFC3031], e em alguns casos pode diferenciar um pouco desta arquitetura devido ao fato do GMPLS também considerar planos de dados não baseados em pacotes. O GMPLS suporta as interfaces e elementos de comutação: PSC (Packet Switching Capable), TSC (TDM - Time Division Multiplexing Switching Capable), LSC (Lambda Switching Capable) e FSC (Fiber Switching Capable).

\footnotetext{
${ }^{5}$ Em outubro, 2002, IDC estimou que o tráfego de Internet aproximadamente dobraria a cada ano nos próximos cinco anos.

${ }^{6}$ Em abril, 2003, RHK previu que o IP teria seu domínio aumentado no volume total de tráfego na rede de $50 \%$ (atual) para aproximadamente 80\% até 2006.
} 
Hoje, baseado nos rígidos requisitos das operadoras, a maior parte dos esforços GMPLS estão direcionados às extensões dos protocolos baseados em IP/MPLS para redes orientadas a circuito de controle, incluindo redes ópticas. GMPLS utiliza o mesmo conjunto de protocolos de sinalização e roteamento utilizada pelas redes IP/MPLS reduzindo assim a complexidade de projeto, desenvolvimento e manutenção. GMPLS também fornece capacidades e características, tais como descobrimento e controle de recursos distribuídos em tempo real (para o uso de um recurso on-line durante a comutação de pacotes), recuperação e estabelecimento de conexão de múltiplas camadas (evitando o uso de várias instâncias de plano de controle antes de estabelecer e recuperar tais conexões), provisionamento de circuito rápido, flexibilidade de serviço tal como serviços de largura de banda sob demanda, melhor interoperabilidade dos elementos de rede de diferentes fabricantes, e aumento da capacidade de sobrevivência fornecendo habilidade de reroteamento dinâmico quando uma falha ocorre, facilitando assim, as operações do dia-a-dia das operadoras [LANDOLSI, 2002], [PAPADIMITRIOU, 2003], [ROUSSEAU, 2003].

Algumas das importantes características do MPLS Generalizado são [PAPADIMITRIOU, 2003]:

- Descobrir os vizinhos de um nó

- Distribuir informações sobre os enlaces

- Fornecer topologia da rede

- Gerenciar caminhos

- Proteger/restaurar enlaces 
Além disso, o GMPLS permite um plano de controle comum, provisionamento automático, balanceamento de carga, largura de banda sob demanda ${ }^{7}$ e OVPN (Optical Virtual Private Network)

Um impacto econômico importante do GMPLS é fornecer a habilidade de automatizar o gerenciamento de recursos da rede e o provisionamento de serviço de caminhos TE fim-a-fim. O provisionamento de serviços é um processo manual que consome muito tempo e, além disso, é um processo de alto custo. O desenvolvimento de nós baseados no GMPLS permite que operadoras automatizem o provisionamento e gerenciamento da rede, aumentem o número de serviços oferecidos aos clientes e prometem reduzir os custos operacionais.

O efeito colateral mais interessante para uma rede GMPLS fim-a-fim é que todo caminho pode ser efetivamente automatizado. Teoricamente, baseado em um conjunto de requisitos, a rede pode estabelecer conexões fim-a-fim sem requerer ao operador o provisionamento em cada nó intermediário. Portanto, tem-se economia de tempo, eliminação de muitos erros, menor custo e potencialmente elevados rendimentos para o operador de rede [CHOI, 2003].

\subsection{EVOLUÇÃO EM DIREÇÃO AO GMPLS}

\subsubsection{MPLS e o Paradigma Label Switching}

Em redes IP, pacotes são encaminhados com base no endereço IP de destino contido no cabeçalho do pacote e também com base na informação armazenada na base de dados de envio do roteador (chamada de base de informação de envio - FIB, do inglês, Forwarding

\footnotetext{
${ }^{7}$ Largura de banda sob demanda é o fornecimento de conectividade entre dois pontos de acesso de uma maneira rápida, automática e de modo não planejado anteriormente, através de sinalização.
} 
Information Base). A FIB contém uma lista com os endereços de rede que são conhecidos pelo roteador e a interface sobre a qual um pacote deve ser encaminhado para com sucesso alcançar o seu destino. Quando um roteador IP recebe um pacote IP, ele realiza uma associação de prefixo longa com o endereço IP de destino do pacote e os endereços de rede contidos na FIB e, envia o pacote através da interface correspondente. Esta associação de prefixo longa é um processo relativamente lento, especialmente quando a FIB contém uma grande quantidade de entradas bem como, parâmetros de qualidade de serviço (QoS - Quality of Service). Um novo paradigma de envio foi desenvolvido com o objetivo de resolver este problema: label switching, no português, troca de rótulos [SAHA, 2002].

No label switching, um rótulo é atribuído a cada pacote e, o encaminhamento do pacote é baeado neste rótulo. Cada roteador - no label switching, conhecido como roteador label switching (LSR - Label Switching Router) - mantém uma base de dados de envio que mapeia um par interface/rótulo de entrada a um par interface/rótulo de saída. Este princípio é ilustrado na Figura 2.1 [ROUSSEAU, 2003].

O fato de que o princípio label switching poderia ser aplicado a diferentes tecnologias de comutação de pacotes/células/frames (IP, ATM - Asynchronous Transfer Protocol, FR Frame Relay) foi importante para o desenvolvimento do conjunto de protocolos MPLS (Multiprotocol Label Switching) [RFC3031].

O caminho utilizado pelos pacotes rotulados é chamado caminho label switching (LSP - Label Switching Path). Os LSRs ao longo de um LSP fazem com que as decisões de envio sejam baseadas nos rótulos transportados pelos pacotes ao invés de serem baseadas no endereço IP de destino, eliminando assim, a associação de prefixo longa. 


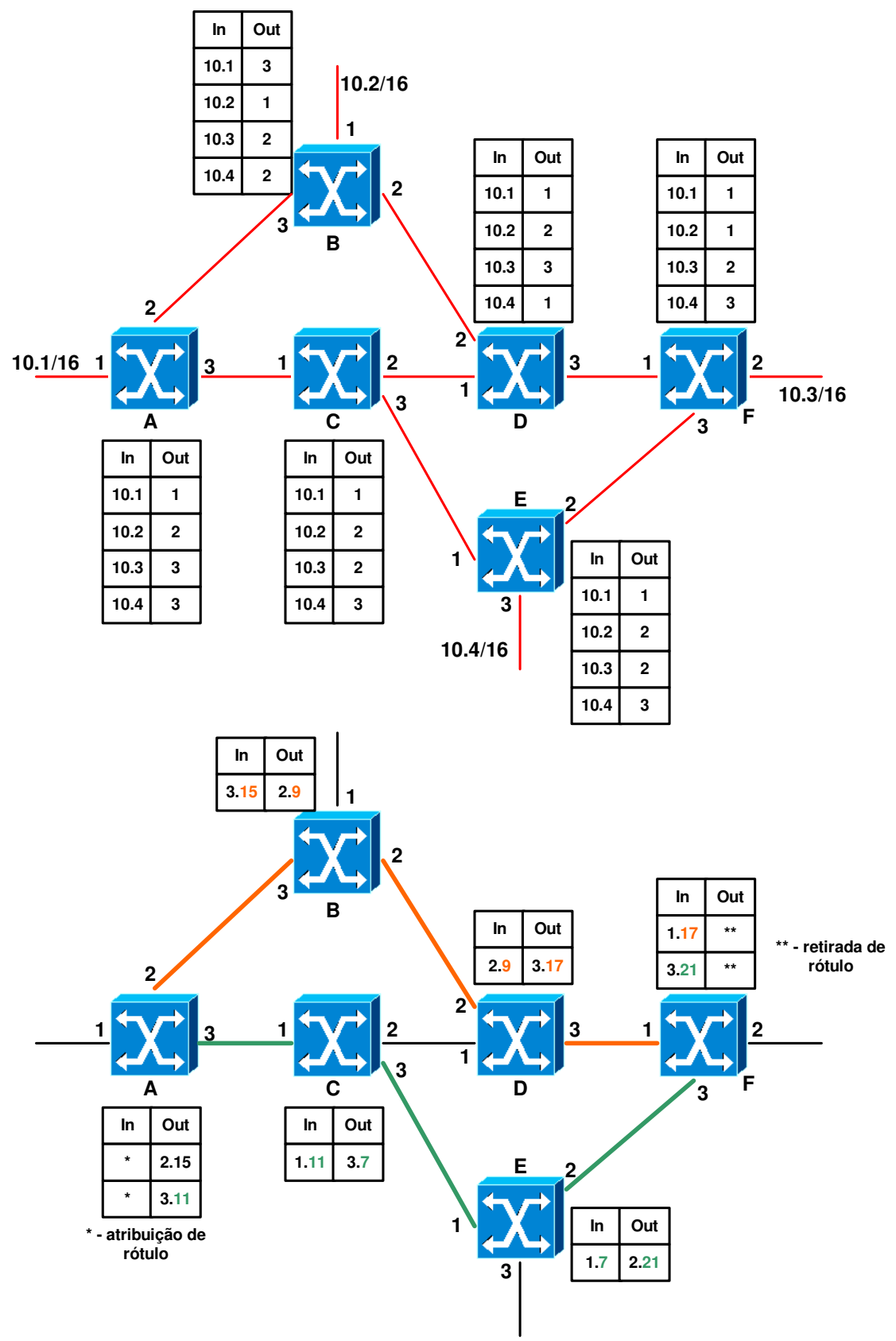

Figura 2.1 - Comparação entre o roteamento IP convencional (topo) e o Paradigma Label Switching (base).

A eliminação da associação de prefixo longa, juntamente com o fato de que existem poucos rótulos para serem buscados na FIB se comparados com o número de endereços IP, tornam o paradigma label switching mais rápido do que o encaminhamento IP tradicional. Note que o 
rótulo atribuído ao pacote pelo roteador de ingresso junto com a informação armazenada nas bases de dados de envio dos LSRs completamente estabelecem o LSP.

\subsubsection{MPLS-TE}

Engenharia de tráfego (TE - Traffic Engineering) é utilizada para otimização do desempenho da rede. $\mathrm{O}$ suporte às capacidades $\mathrm{TE}$ aprimoradas requerem não somente protocolos de sinalização estendidos tal como RSVP-TE (Resource Reservation Protocol) [RFC3209], mas também versões estendidas dos protocolos de roteamento tais como OSPF (Open Shortest Path First) [RFC2328] e ISIS (Intermediate System to Intermediate System) [RFC1195]. Isto gerou os protocolos OSPF-TE e ISIS-TE que fornecem informações TE mais detalhadas sobre o status e utilização dos recursos de rede. Estas extensões de engenharia de tráfego ao MPLS são referidas como MPLS-TE [CONTE, 2003], [IOVANNA, 2003].

Observa-se que embora o label switching tenha sido originalmente introduzido para melhorar a velocidade de encaminhamento (eliminado a demorada associação de prefixo longa), o foco foi direcionado para o aprimoramento da flexibilidade e capacidades de QoS e para mais flexíveis e eficientes mecanismos de controle (devido ao aprimoramento das capacidade TE).

\subsubsection{MP $\lambda S$}

Quando as tecnologias ópticas foram introduzidas nas redes de telecomunicação, elas foram desenvolvidas como enlaces ponto-a-ponto interconectando dispositivos como crossconnects. Com o surgimento do WDM/DWDM, foi possível multiplexar diversos comprimentos de onda em uma única fibra. Os sistemas DWDM foram inicialmente aplicados 
de forma a exigir operações manuais para interconexão de fibras, tornando impossível qualquer flexibilidade e eliminando a granularidade de todo o sinal multiplexado.

Devido a crescente importância do tráfego IP/MPLS associado com sua dinâmica de tráfego, a reconfiguração de conexão óptica tornou mais freqüente e a realização desta operação manualmente não era mais satisfatória. Portanto, para atender as mudanças das condições de tráfego de maneira conveniente, eliminando o enorme tempo despendido e as operações manuais possíveis causadoras de erros, cross-connects ópticos foram introduzidos para fornecer capacidades cross-connect automatizadas e controladas remotamente. O conceito de comutação por comprimento de onda, ou comutação lambda assim surgiu [ROUSSEAU, 2003].

Logo tornou-se claro que apenas o cross-connect automatizado e controlado remotamente é insuficiente para suportar aplicações de demanda tal como recuperação rápida de rede em caso de falhas: um plano de controle distribuído é necessário. O IETF iniciou a padronização deste plano de controle no final dos anos 90 [MP $\lambda \mathrm{S}]$. Baseado na idéia de que um comprimento de onda pode ser considerado equivalente a um rótulo, o conjunto de protocolos MPLS foi estendido para comutação lambda; e assim surgiu o MP $\lambda$ S. Enquanto no MPLS um par interface/rótulo de entrada é associado a um par interface/rótulo de saída, no MP $\lambda \mathrm{S}$ um par fibra/comprimento-de-onda de entrada é mapeado a um par fibra/comprimento-de-onda de saída [GALLAHER, 2001].

Depois que o trabalho de definição do MP $\lambda \mathrm{S}$ foi inicializado, percebeu-se que o paradigma label switching poderia não apenas ser aplicado a pacotes/células/frames e comprimentos de onda, mas este poderia ser generalizado para slots de tempo, faixa de ondas, fibras, etc. Como 
consequiência desta generalização, o MP $\lambda S$ foi aprimorado gerando assim o MPLS generalizado (GMPLS - Generalized MPLS) [SAHA, 2002].

\subsubsection{Conceitos Fundamentais do GMPLS}

Como mencionado anteriormente, o GMPLS evolui a partir do MPLS via MP $\lambda S$ e estendeu o paradigma label switching de tecnologias de comutação de pacotes/células/frames para tecnologias orientadas à conexão [IEC], [DEO, 2003].

Os seguintes objetivos de projeto foram mencionados quando GMPLS foi definido [DEO, 2003], [PAPADIMITRIOU, 2003], [ROUSSEAU, 2003]:

- Redução do número de camadas de comutação

- Re-uso do paradigma de comutação de rótulos

- Re-uso do conjunto de protocolos e mecanismo MPLS-TE

- Re-uso do método de endereçamento IP

Importante neste contexto é que o GMPLS extensivamente re-utiliza os conceitos e protocolos introduzidos pelo MPLS. O GMPLS se baseia nas seguintes inovações técnicas para atender os objetivos de projeto citados [PAPADIMITRIOU, 2003], [ROUSSEAU, 2003]:

- Hierarquia LSP

Este conceito consiste do fato de LSPs de diferentes interfaces de controle serem colocados dentro de outro LSP com o mesmo tipo de interface ou com um tipo diferente de interface, obtendo assim, uma hierarquia de envio. Esta hierarquia pode ser construída se uma interface for capaz de multiplexar diversos LSPs de uma mesma camada, mas 
também, pode ocorrer entre interfaces de diferentes tipos como mostra a Figura 2.2 [MANNIE, 2004], [DEO, 2003], [PAPADIMITRIOU, 2003].

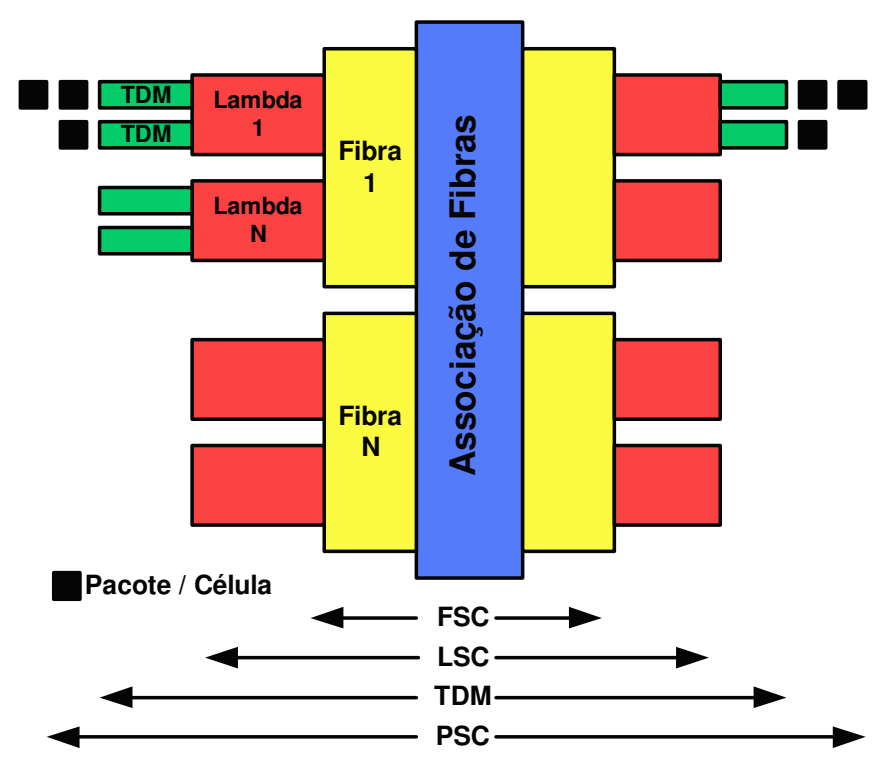

Figura 2.2 - Hierarquia de Envio.

Na hierarquia de envio, muitos LSPs de engenharia de tráfego (LSPs-TE) são agregados dentro de um LSP-TE maior. Nós intermediários veêm somente o LSP externo. Eles não precisam manter os estados de envio para cada LSP interno. Logo, uma quantidade menor de mensagens de sinalização é necessária e assim a escalabilidade de sinalização aumenta.

- LSPs bidirecionais

Um LSP bidirecional possui os mesmos requisitos TE incluindo compartilhamento, proteção e restauração, LSRs, e requisitos de recursos (e.g. latência e jitter), em cada direção. Quando o MPLS(-TE) é utilizado para estabelecer um LSP bidirecional, dois LSPs unidirecionais devem ser independentemente configurados. 
O GMPLS suporta LSPs bidirecionais com estabelecimento simultâneo do caminho downstream e do caminho upstream utilizando um único conjunto de mensagens de sinalização. Para LSPs bidirecionais, dois rótulos devem ser alocados, um para o caminho downstream (requerido pelo nó upstream e atribuído pelo nó downstream) e outro para o caminho upstream (como especificado pelo nó upstream), como mostra a Figura 2.3. O estabelecimento de um LSP bidirecional no GMPLS é indicado pela presença de um rótulo upstream em uma mensagem de sinalização apropriada [DEO, 2003], [PAPADIMITRIOU, 2003].

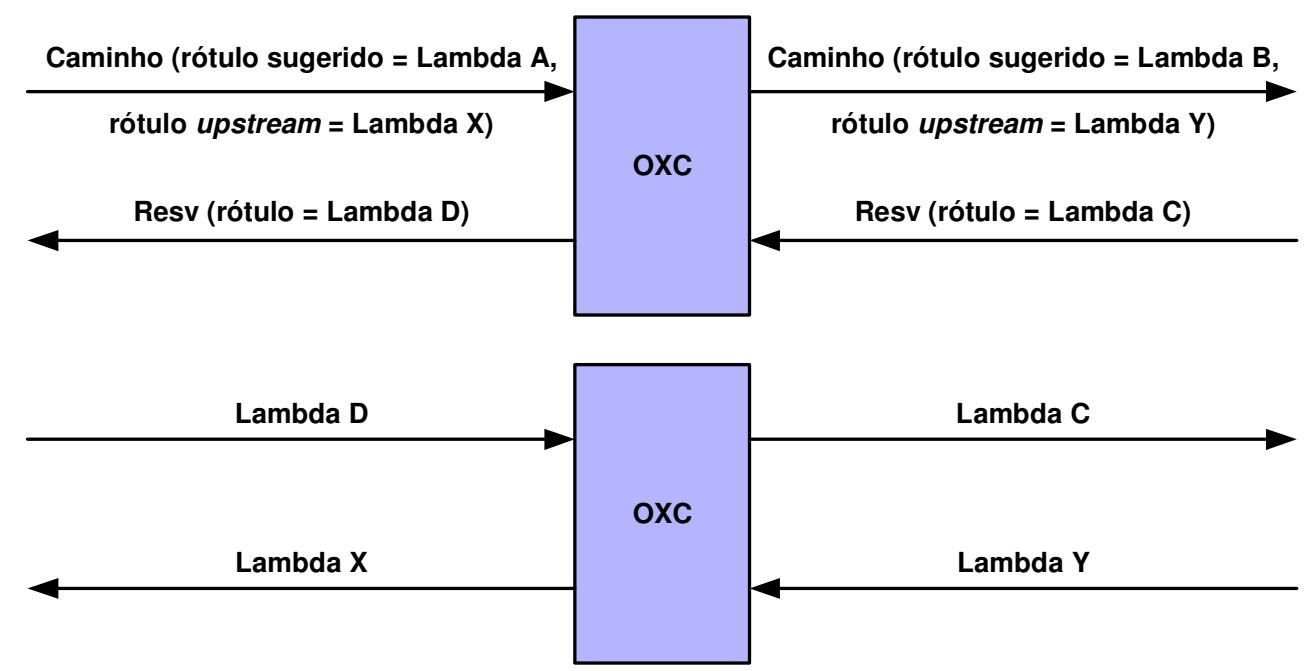

Figura 2.3 - Alocação de Rótulos para LSPs Bidirecionais com Estabelecimento Simultâneo.

- Aprimoramento das capacidades de roteamento IGP-TE (Interior Gateway Protocol - Traffic Engineering)

- Enlaces não-numerados

Enlaces não numerados são usados para aumentar a escalabilidade de endereçamento. Definidos como ponto-a-ponto, estes enlaces ou interfaces não são identificados por um endereço IP, ao contrário, eles utilizam a combinação de um identificador de roteador único e um número de enlace, que unicamente 
identifica o enlace em um escopo local da entidade de plano de controle. Esta combinação é de 32 bits e é chamada de identificador local de enlace, da perspectiva do LSR host, ou identificador remoto de enlace, da perspectiva dos demais LSRs. Protocolos de sinalização e protocolos de roteamento foram estendidos para transportarem e concordarem com tal informação no plano de sinalização [MANNIE, 2004], [IEC].

- Enlaces TE

Quando um par de LSRs é conectado por múltiplos enlaces, é possível anunciar vários ou todos estes enlaces como um único enlace no OSPF e/ou ISIS. Este processo é chamado associação de enlaces. O enlace lógico resultante é chamado enlace TE e seus enlaces físicos são chamados de enlaces componentes [DEO, 2003]. A finalidade da associação de enlace é melhorar a escalabilidade de roteamento através da redução da quantidade de informação que deve ser manipulada pelo IGP, pois o mesmo enxerga somente o enlace TE, e não cada enlace componente. Esta redução é atingida pela agregação/abstração da informação. [MANNIE, 2004], [PAPADIMITRIOU, 2003].

- Protocolo de gerenciamento de enlace

Mencionado na seção 2.5.3 e detalhado no Capítulo V.

\subsection{RÓTULOS GENERALIZADOS}

MPLS Generalizado difere do MPLS tradicional pois suporta múltiplos tipos de comutação. Para lidar com este amplo escopo de domínios de comutação e provisionar um caminho ótimo 
baseado nos requisitos dos diferentes tipos de tráfego do usuário, várias novas formas de rótulos são requeridas. Estas novas formas de rótulos são coletivamente referidas como “rótulo generalizado" [MANNIE, 2004], [IEC].

O rótulo generalizado é uma extensão do rótulo tradicional permitindo a representação de não somente rótulos que viajam com os pacotes de dados associados, mas também rótulos que identificam slots de tempo, comprimentos de onda ou fibra (espaço físico).

Os rótulos generalizados podem ser classificados como [CHOI, 2003], [PAPADIMITRIOU, 2003]:

- Rótulos de Slots de Tempo

A largura de banda de uma fibra óptica é subdividida em slots de tempo pela multiplexação por divisão de tempo (TDM - Time Division Multiplexing) e, um comutador óptico pode satisfazer um pedido de fluxo de dados particular alocando um ou mais slots de tempo para este fluxo.

- Rótulos de Comprimento de Onda

A largura de banda de uma fibra óptica é subdividida através de multiplexação por divisão de comprimento de onda (WDM - Wavelength Division Multiplexing). Um LSR óptico pode alocar um único comprimento de onda para um fluxo de dados pedido. Neste caso, o valor do rótulo é o comprimento de onda selecionado.

- Rótulos de Faixa de Ondas

Se comprimentos de onda consecutivos são agrupados em uma faixa de ondas logo, para que todos sejam comutados da mesma forma, o rótulo é composto por um identificador de faixa de ondas e um par de números inteiros e positivos (identificadores de canais) indicando o comprimento de onda inferior e superior da faixa de ondas selecionada. 
- Rótulos de Fibra

Roteadores Label Switching (LSRs) podem alocar toda uma fibra para um fluxo de dados e assim simplesmente precisam concordar em qual fibra usar. Neste caso, o valor do rótulo utilizado é o número da fibra selecionada. A interpretação dos números de fibra/porta é um problema local para os LSRs no enlace.

O tamanho dos rótulos generalizados é de 32 bits como mostra a Figura 2.4a. O rótulo de faixa de ondas é uma exceção; este é formado por um maior número de bits (96 bits) por ser composto por um maior número de informações: 32 bits do ID (identificador) de faixa de ondas, 32 bits do comprimento de onda inferior e 32 bits do comprimento de onda superior, como apresentado na Figura 2.4b.

Como os nós enviando e recebendo as novas formas de rótulos sabem que tipo de enlace eles estão usando, o rótulo generalizado não contém um campo “tipo”, ao contrário, espera-se que os nós saibam, com base no contexto, o tipo de rótulo esperado.

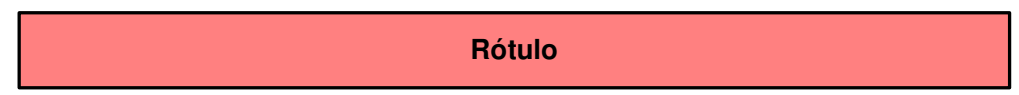

(a)

\begin{tabular}{|c|}
\hline ID de Waveband \\
\hline Comprimento de Onda Inferior \\
\hline Comprimento de Onda Superior \\
\hline
\end{tabular}

(b)

Figura 2.4 - Rótulos Generalizados

Para todos os tipos de domínios descritos, o valor do rótulo implica diretamente a largura de banda disponível para o fluxo de dados correspondente. Esta situação é um pouco diferente do 
caso dos rótulos não-generalizados e é uma reflexão fundamental da natureza das redes ópticas.

\subsubsection{Pedido de Rótulo Generalizado}

Os procedimentos do protocolo MPLS básico para estabelecimento do valor do rótulo através de um enlace não são diferentes para as redes ópticas:

- O LSR upstream envia um pedido ao LSR downstream (uma mensagem Path no RSVP, um pedido de rótulo no CR-LDP - Constraint-based Routing Label Distribution Protocol). O pedido contém informações suficientes sobre a largura de banda e qualidade de serviço requeridos para que o LSR downstream faça uma escolha de rótulo adequada.

- O LSR downstream recebe o pedido e aloca o valor de um rótulo que atende os requisitos específicos do pedido.

- O LSR downstream envia uma resposta ao LSR upstream (Resv no RSVP, Mapeamento de rótulo no CR-LDP) comunicando o valor de rótulo selecionado.

Algumas informações que o LSR downstream precisa, com o objetivo de realizar a melhor escolha para o valor do rótulo requerido, são indiretamente sugeridas pelo contexto. No nível mais básico, ambos LSRs envolvidos sabem que o rótulo deve ser um rótulo generalizado ao invés de um rótulo não-generalizado, pois o enlace ao qual o pedido se relaciona é um enlace óptico generalizado. Portanto, esta informação não está explícita na mensagem de pedido.

No entanto, como um enlace óptico pode ser formado por um conjunto de fibras, e os comutadores podem suportar mais de um tipo de multiplexação nestas fibras, é necessário que o LSR upstream especifique o tipo de codificação do LSP que ele quer para o fluxo de dados 
sendo estabelecido; este tipo de codificação então determina se o rótulo selecionado é baseado em slot de tempo ou comprimento de onda, por exemplo, e de que tipo.

O pedido de rótulo generalizado deve ser adicionado na mensagem Path RSVP-TE ou, em uma mensagem de pedido CR-LDP. Somente um pedido de rótulo generalizado pode ser usado em cada mensagem, logo um único LSP pode ser requerido por cada mensagem de sinalização.

O pedido de rótulo generalizado realiza a comunicação das características requeridas para suportar o LSP sendo pedido, como mencionado anteriormente. Outros parâmetros específicos de tecnologia não são transportados no pedido de rótulo, mas sim em parâmetros de tráfego específicos da tecnologia.

\subsubsection{Escolha de rótulos com restrições}

Como descrito anteriormente, a escolha do rótulo para cada enlace é normalmente feita pelo nó downstream daquele enlace específico. No MPLS não-generalizado, onde rótulos são simplesmente números arbitrários, este procedimento não resulta em nenhum tipo de problema ao ambiente de rede. No MPLS Generalizado, onde os rótulos são diretamente

relacionados com os recursos da rede, esta escolha pode levar a conflitos durante o estabelecimento do LSP. Por exemplo, um comutador óptico baseado em micro espelhos pode ser capaz de comutar um comprimento de onda de uma porta de entrada a uma porta de saída, mas não é capaz de modificar o comprimento de onda. 
A Figura 2.5 apresenta dois LSPs em uma rede óptica onde os comutadores (OXCs - Optical Cross Connects) são incapazes de fazer conversões de comprimento de onda. O LSP-L1 (comprimento de onda L1 é usado) passa pelos nós A, B, D, E e F. O LSP-L2 é estabelecido entre os nós C, D, E e G (cores representam os comprimentos de onda usados pelos LSPs).

Não há conflitos no enlace entre os nós D e E pois os dois LSPs utilizam comprimentos de onda diferentes. No entanto, quando o LSR C precisa estabelecer um novo LSP através dos nós D, E e G, este deve escolher um novo comprimento de onda. Se a escolha for realizada pelo LSR G (o que é o procedimento normal no GMPLS), o nó G poderia escolher o comprimento de onda L1. Como o comprimento de onda L1 já está sendo utilizado entre os nós D e E, o mesmo não pode ser utilizado. Se a escolha for feita pelo LSR C, um problema similar pode acontecer [JERRAN, 2001].

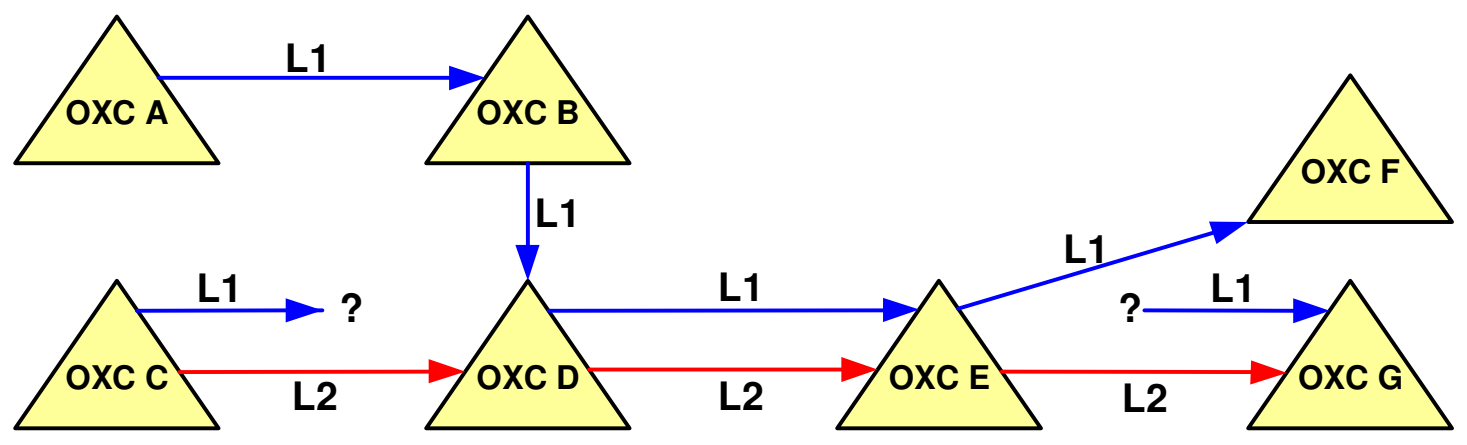

Figura 2.5 - Conflito na Escolha de Rótulos com Restrição em Comutadores Ópticos.

Existe, portanto, uma necessidade em permitir que todos os OXCs ao longo de um caminho a ser estabelecido influenciem e/ou restrinjam a escolha de rótulos para garantir que os rótulos apropriados sejam escolhidos.

GMPLS introduz o conceito de conjunto de rótulos para restringir a faixa dos rótulos que podem ser usados por um LSP particular entre dois peers. Um LSR upstream inclui um 
conjunto de rótulos no seu pedido de sinalização para restringir a escolha de um rótulo pelo LSR downstream para o enlace entre eles. O receptor de um conjunto de rótulos (LSR downstream) limita sua escolha de rótulos para um rótulo pertencente ao conjunto de rótulos caso contrário o estabelecimento do LSP falha (esta limitação se aplica a cada salto, pois semelhante a um rótulo, um conjunto de rótulos deve estar presente através de múltiplos saltos) [MANNIE, 2004].

Com o objetivo de suportar o objeto conjunto de rótulos, controladores de comutador óptico (OSCs - optical switch controllers) possuem dois reservatórios de comprimentos de onda denominados Reservatório dos Utilizados (UP - Used Pool) e Reservatório dos Disponíveis (AP - Available Pool). O AP é usado para sugerir comprimentos de onda para downstream. Se qualquer comprimento de onda no conjunto de rótulos recebido está no UP, o comprimento de onda específico é retirado do conjunto de rótulos antes de ser encaminhado ao nó downstream. Por exemplo, um LSR que é incapaz de realizar conversão de comprimentos de onda gera um conjunto de rótulos de saída para encaminhar o conjunto de rótulos de entrada menos qualquer rótulo que ele não pode gerar ou receber [BERGER, 2003].

Considere novamente o exemplo na Figura 2.5. Suponha que o LSR C pode somente gerar comprimentos de onda na faixa R. Assim, ele sinaliza um conjunto de rótulos de "qualquer comprimento de onda na faixa R exceto L2". LSR D modifica este conjunto e sinaliza "qualquer comprimento de onda na faixa R exceto L2 e L1". LSR E encaminha esta sinalização e LSR G pode selecionar um comprimento de onda que é aceitável a todos os LSRs no caminho, se um estiver disponível. 
O conjunto de rótulos é útil em domínios ópticos quando, por exemplo:

- Um LSR é incapaz de converter diferentes comprimentos de onda

- Um LSR pode somente gerar e receber um subconjunto de comprimentos de onda que podem ser comutados pelos LSRs vizinhos

- É desejável por um LSR que a quantidade de conversões de comprimentos de onda que devem ser realizadas seja limitada, com o objetivo de reduzir distorções nos sinais ópticos.

- Duas terminações de enlace suportam diferentes conjuntos de comprimentos de onda

Segue então a descrição do estabelecimento de um caminho óptico no GMPLS utilizando o conceito de conjunto de rótulos.

A sinalização GMPLS estabelece um caminho óptico da origem ao destino, assumindo que as extensões GMPLS para RSVP-TE são utilizadas. A abordagem de sinalização segue o modo downstream sob demanda onde um nó upstream faz um pedido de rótulo explicitamente e um nó downstream atribui um rótulo particular como resposta ao pedido. Além disso, um nó somente atribui um rótulo ao seu vizinho upstream se o mesmo for o nó de destino, ou se este já recebeu um rótulo do seu próximo salto. Assim, a fase de sinalização consiste de um pedido de rótulo que passa nó a nó da origem ao destino, seguido por uma atribuição de rótulo encaminhada ao longo da rede em direção oposta ao pedido, de volta a origem. No RSVP-TE, um pedido de rótulo está contido na mensagem Path enquanto que a atribuição de rótulo está contida na mensagem Resv [OKI, 2004].

Quando a origem precisa estabelecer um caminho óptico para um destino em particular, primeiro ela determina a rota/caminho em direção ao destino. Assim que a rota for 
determinada, a origem inicia uma mensagem Path (pedido de rótulo generalizado) do RSVP$\mathrm{TE}^{8}$ contendo:

- objeto de pedido de rótulo

- objeto de rota explícita (ERO - explicit route object) que armazena a rota que foi determinada

- objeto conjunto de rótulos que permite que o nó upstream restrinja o conjunto de rótulos que o nó downstream pode selecionar e,

- outros objetos relevantes.

À medida que o conjunto de rótulos se propaga na mensagem Path, cada nó downstream pode gerar um novo conjunto de rótulos de saída possivelmente baseado no conjunto de rótulos de entrada e na sua capacidade de hardware. Quando o nó de egresso (destino) recebe a mensagem Path, ele seleciona qualquer rótulo pertencente ao conjunto de rótulos de entrada, e envia sua escolha em um objeto reserva de rótulos usando a mensagem Resv. Cada nó ao longo da rota reserva o rótulo selecionado à medida que a mensagem Resv segue em direção ao nó de ingresso [KIM, 2003].

Se o nó é incapaz de escolher um rótulo do conjunto de rótulos ou se existe um problema em analisar o conjunto de rótulos, então o pedido é finalizado e uma mensagem PathErr com uma indicação "problema de roteamento/ conjunto de rótulos" deve ser gerada. Quando o conjunto de rótulos resultante é vazio, a mensagem Path deve ser finalizada, e uma mensagem PathErr e uma indicação "problema de roteamento/ conjunto de rótulos" deve ser gerada.

${ }^{8}$ Os termos do protocolo RSVP-TE são utilizados e os termos do protocolo CR-LDP são fáceis de serem deduzidos. 
A Figura 2.6 ilustra a sequiência de passos necessários para o estabelecimento de um caminho óptico. No passo (a), a origem inclui um conjunto de rótulo $\left\{\lambda_{1}, \lambda_{2}, \lambda_{3}, \lambda_{4}, \lambda_{5}, \lambda_{6}\right\}$ na sua mensagem Path indicando que o laser da origem é capaz de sintonizar seus comprimentos de onda para qualquer um dos seus comprimentos de onda possíveis. Após o recebimento da mensagem Path, o primeiro roteador de comprimento de onda faz a interseção do conjunto de rótulos de entrada e o conjunto de rótulos disponíveis no seu enlace de saída, resultando em um conjunto de rótulos de saída $\left\{\lambda_{1}, \lambda_{4}, \lambda_{5}, \lambda_{6}\right\}$, que será encaminhado na mensagem Path ao próximo salto. Cada roteador de comprimento de onda realiza a mesma operação até que o conjunto de rótulos seja propagado ao destino, que então seleciona um dos rótulos aceitáveis. Depois que o destino selecionou um rótulo indicado no passo (b), rótulo $\lambda_{1}$ é atribuído a cada salto em direção a origem. Quando todos os rótulos ao longo do caminho forem atribuídos, um caminho óptico com comprimento de onda $\lambda_{1}$ entre origem e destino é estabelecido (passo (c)) [WIDJAJA, 2002], [OKI, 2004], [KIM, 2003].

(a)
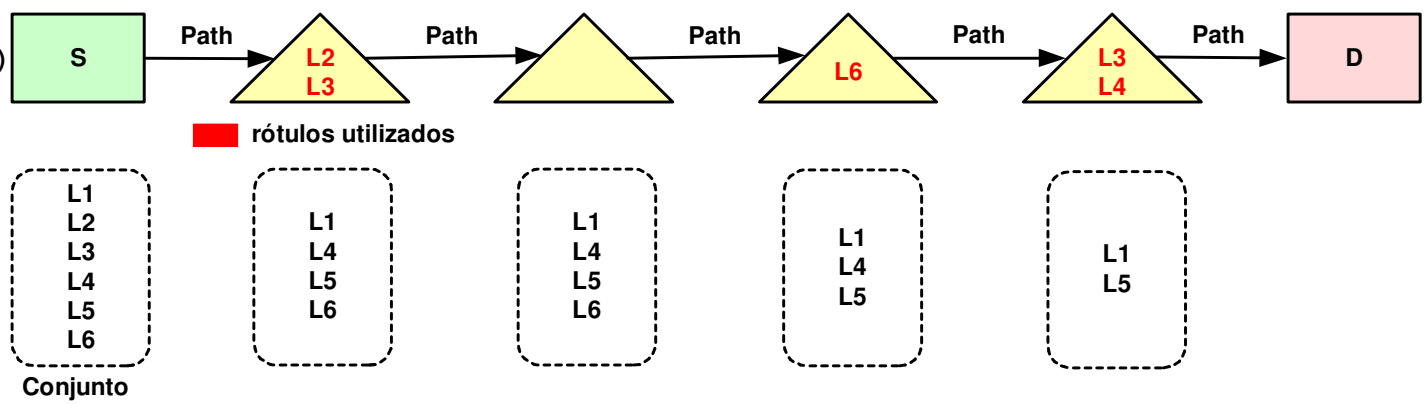

rotulos utilizados

de Rótulos
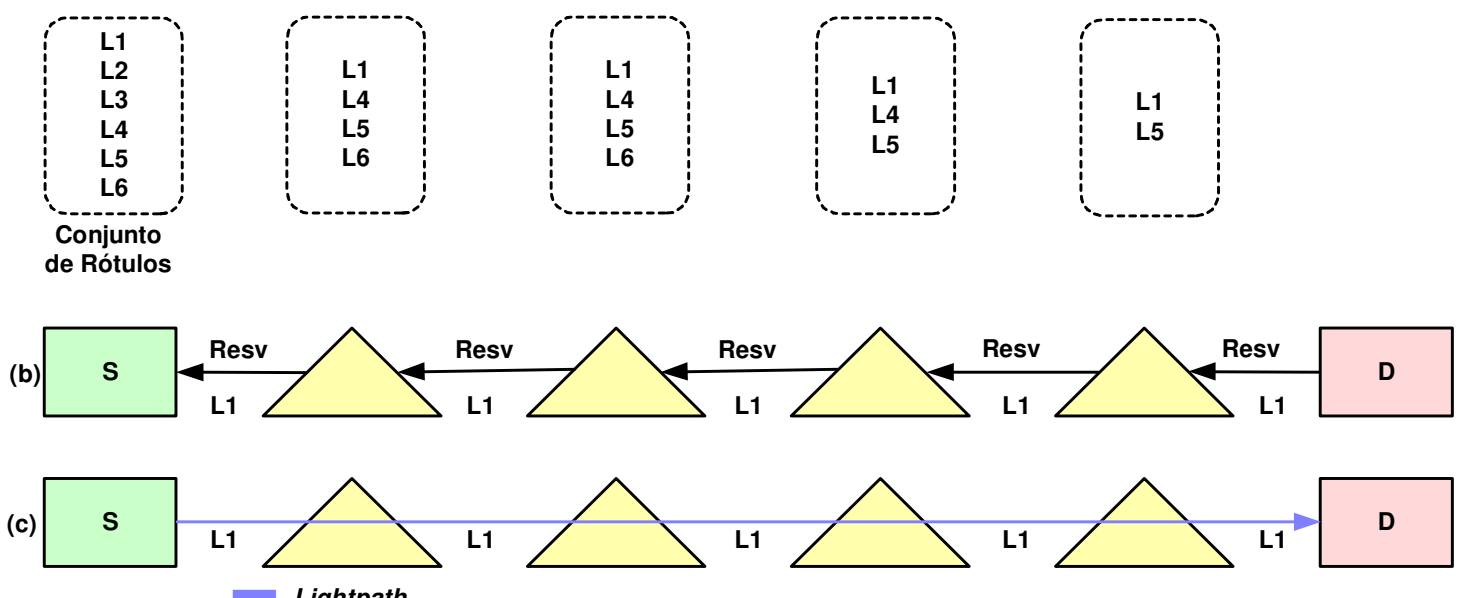

D

Figura 2.6 - Como estabelecer um caminho óptico com sinalização RSVP-TE. 
Note que um rótulo pode ser sugerido por múltiplas mensagens Path, pois o rótulo não é realmente reservado até que a mensagem Resv seja recebida. Assim, múltiplas mensagens Resv podem tentar reservar os mesmos recursos, resultando em falha para pelo menos um pedido de conexão e conseqüentemente, uma mensagem ResvErr deve ser enviada aos nós do caminho que já realizaram a reserva do comprimento de onda selecionado.

Quando há demanda em uma rede GMPLS total ou parcialmente óptica e é preciso realizar roteamento e seleção de comprimento de onda, um pedido de conexão pode falhar devido a um dos dois eventos de bloqueio denominados bloqueio de enlace forward e bloqueio de enlace backward. O bloqueio de enlace forward é devido a recursos de comprimento de onda insuficientes e a não utilização de algoritmos de roteamento que promovem o balanceamento no uso dos enlaces. Bloqueio de enlace backward acontece devido ao conflito de reservas de rótulos (no caso das redes GMPLS ópticas, um rótulo particular é sinônimo de um comprimento de onda particular). Assim, o objeto conjunto de rótulos não é suficiente para eliminar o bloqueio de enlace backward que ocorre quando dois ou mais nós de egresso selecionam o mesmo rótulo para conexões que compartilham os mesmos enlaces.

\subsection{INTERFACES SUPORTADAS}

A arquitetura MPLS foi estendida de forma que as decisões de comutação na rede possam ser tomadas com base não somente nos cabeçalhos dos pacotes e células mas também com base em slots de tempo, comprimentos de onda e espaços físicos. Assim, as interfaces LSRs suportadas no GMPLS, apresentadas na Figura 2.7, são [POLARIS], [MANNIE, 2004], [IEC], [DEO, 2003]: 
- $\quad$ PSC - Packet Switch Capable

Interfaces com capacidade de comutação de pacotes. O encaminhamento de dados é baseado no conteúdo do cabeçalho MPLS transportado por pacotes e células.

\section{- $\quad$ TSC - Time Division Multiplexing Switch Capable}

Interfaces com capacidade TDM. As decisões de envio são baseadas no slot de tempo dos dados em um ciclo repetitivo.

\section{- $\quad$ LSC - Lambda Switch Capable}

Interfaces com capacidade de comutação de lambdas (ou comprimento de ondas). O envio de dados se baseia no comprimento de onda ou faixa de comprimentos de onda (waveband) no qual os dados são recebidos.

\section{- $\quad$ FSC - Fiber Switch Capable}

Interfaces com capacidade de comutação de fibras. $\mathrm{O}$ encaminhamento de dados é baseado na posição dos dados no espaço físico do mundo real (e.g., porta de entrada ou fibra).

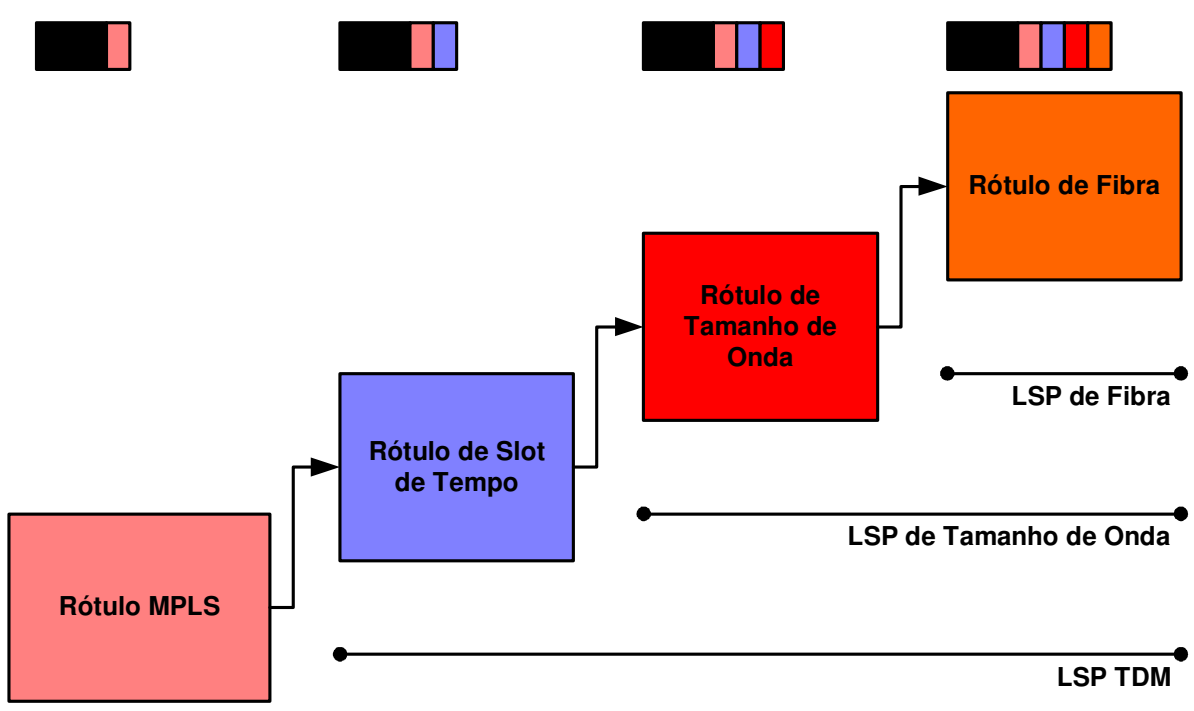

Figura 2.7. Interfaces suportadas pelo GMPLS. 


\subsection{CONJUNTO DE PROTOCOLOS}

A arquitetura do plano de controle GMPLS (Figura 2.8) é composta por diferentes blocos [LI, 2002]. Eles são baseados nos protocolos de sinalização e roteamento MPLS-TE que foram estendidos para suportar o GMPLS, bem como no proposto protocolo de gerenciamento de enlace [MANNIE, 2004], [ABOUL-MAGD, 2001], [IEC], [PAPADIMITRIOU, 2003].

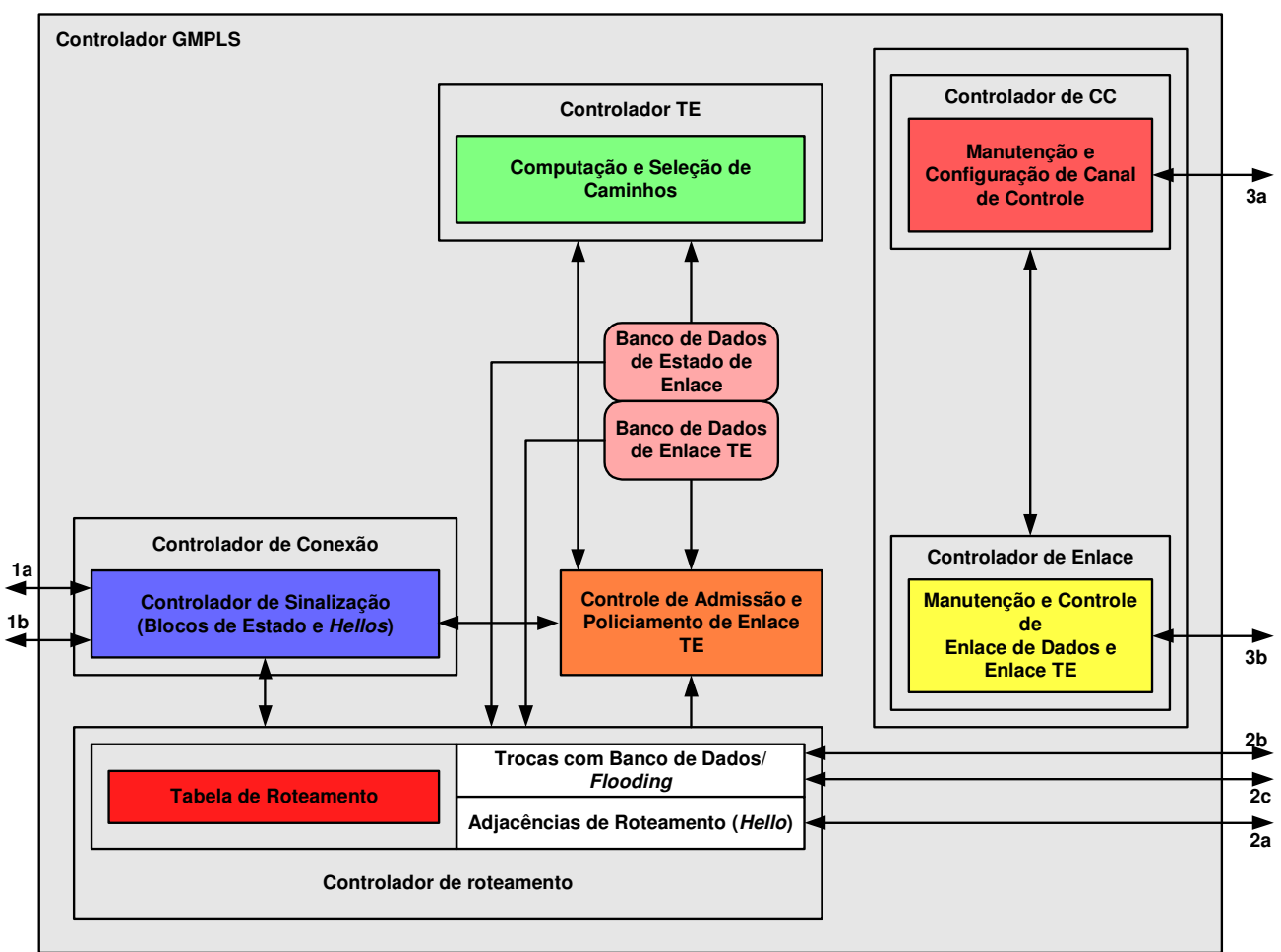

Figura 2.8 - Fluxo de Informação e Controle GMPLS.

A Figura 2.8 resume os diferentes fluxos externos de informação e as interações internas de plano de controle entre os distintos blocos da arquitetura de plano de controle GMPLS:

- Sinalização - sinalização RSVP-TE (1a) e Hellos (1b) para manutenção de adjacência de sinalização 
- Roteamento - OSPF-TE com Hellos para descobrimento e manutenção de adjacência de roteamento (2a), troca de descrição de banco de dados (2b) e, atualização/pedido/ reconhecimento de estado de enlace $(2 \mathrm{c})$

- Protocolo de gerenciamento de enlace - manutenção e configuração de canal de controle (Hellos - 3a) e, gerenciamento de enlace de dados e enlace TE (verificação, correlação e gerenciamento de falha - 3b)

\subsubsection{Protocolos de Roteamento}

O roteamento GMPLS [BANERJEE, 01/2001] refere-se à disseminação de informação de alcançabilidade, topologia e recursos/capacidade através da topologia de roteamento do plano de controle. GMPLS fornece extensões de roteamento TE adicionais às interfaces não-PSC, se comparado ao roteamento TE realizado no MPLS-TE através dos protocolos OSPF-TE (Open Shortest Path First - Traffic Engineering) e ISIS-TE (Intermediate System to Intermediate System - Traffic Engineering) [KOMPELLA, 2003].

Para o aprimoramento de escalabilidade, os seguintes mecanismos foram definidos: enlaces não numerados, tipo de proteção de enlace, informação de grupo de enlace de risco compartilhado (SRLG - Shared Risk Link Group) e associação de enlaces (note que estes mecanismos são igualmente aplicáveis às interfaces PSC).

Para utilizar os recursos de forma mais eficiente, o algoritmo de computação de caminhos considera várias restrições TE. Assim, o roteamento baseado em restrições ultrapassa o algoritmo SPF (Shortest Path First) tradicional. Estas restrições incluem restrições intrínsecas (ou de rede) tais como capacidades de enlace TE (framing, proteção, etc) e suas 
disponibilidades de recursos (largura de banda, etc) e, restrições extrínsecas tais como o nível de serviço requerido e tempo médio de recuperação. Em resumo, roteamento baseado em restrições deve considerar as restrições intrínsecas e extrínsecas para computar o(s) melhor(es) caminho(s) que as satisfazem.

Para tornar a computação de caminho baseada em restrições praticável, o LSR que inicia o pedido do LSP precisa de mais informação (i.e., atributos) sobre os enlaces TE do que os protocolos de roteamento intra-domínio fornecem para as redes IP/MPLS. Estes atributos TE são disseminados usando os mecanismos de distribuição dos protocolos de roteamento de estado de enlace já estendidos para o MPLS-TE tais como, OSPF-TE e ISIS-TE. Assim, os algoritmos de computação e seleção de caminhos são mantidos específicos por cada fabricante, desde que os atributos TE sejam semanticamente padronizados [DEO, 2003].

Extensões aos protocolos de roteamento do MPLS-TE são fornecidas para uniformemente codificarem e transportarem informações de enlace TE. Esta informação é então utilizada pelos algoritmos de computação de caminhos para estabelecerem rotas explícitas através do processo de sinalização. Rotas explícitas garantem que as restrições TE e o nível de serviços requeridos (como parte do pedido de conexão) sejam satisfeitos no estabelecimento do LSP ao longo da rede. Enquanto o roteamento explícito é normalmente utilizado, o roteamento saltoa-salto pode ser considerado, mas este tem um escopo de aplicabilidade restrito devido à geração permanente de loops em ambientes altamente dinâmicos [KOMPELLA, 2003]. 


\subsubsection{Protocolos de Sinalização}

A sinalização GMPLS [BANERJEE, 07/2001] estende dois protocolos definidos pelo MPLSTE, RSVP-TE [BERGER, 2003] e CR-LDP, e em alguns casos adiciona funcionalidades. Estas mudanças e adições provocam impacto em propriedades LSP básicas, por exemplo, na forma como os rótulos são requeridos e distribuídos, a natureza unidirecional dos LSPs, a maneira como os erros são propagados e a informação fornecida para sincronização do nó de ingresso e do nó de egresso.

Estes protocolos de sinalização devem ser capazes de transportar os parâmetros LSP requeridos tais como largura de banda, tipo de sinal, proteção de enlace desejada, posição em uma multiplexação específica, etc [BERGER, 2003].

A sinalização GMPLS comanda a alocação de rótulo do tipo downstream sob-demanda bem como a distribuição com controle ordenado e iniciado pelo nó de ingresso. Embora a retenção de rótulo liberal seja normalmente usada, a retenção de rótulos conservativa ${ }^{9}$ também deve ser considerada. Além disso, não há restrição quanto à estratégia de alocação de rótulos. A alocação pode ser orientada a pedido/sinalização (o que é obvio para as tecnologias de comutação de circuito), orientada a topologia ou até mesmo orientada a dados/tráfego (embora esta última opção seja muito comum, ela requer a definição de uma função de ativação) [DEO, 2003].

A sinalização GMPLS define novos blocos de construção no topo do MPLS-TE:

(i) Novo formato de pedido de rótulo genérico

\footnotetext{
${ }^{9}$ Se um LSR suporta o modo de retenção de rótulo liberal, ele mantém as associações entre um rótulo e uma FEC que é recebida de LSRs que não são considerados como próximo salto para a FEC. Se um LSR suporta o modo de retenção de rótulo conservativo, ele descarta tais associações. Mais detalhes em RFC3031.
} 
(ii) Rótulos para as interfaces TSC, LSC e FSC, conhecidos como rótulos generalizados

(iii) Suporte para comutação de faixa de ondas

(iv) Sugestão de rótulo pelo nó upstream para finalidade de otimização (e.g. latência)

(v) Restrição de rótulo pelo nó upstream para suportar algumas restrições ópticas

(vi) Estabelecimento de LSP bidirecional com resolução de contenção

(vii) Extensões de notificação de falha

(viii) Informação de proteção e foco na recuperação de enlace, mais indicação do LSP primário e secundário

(ix) Roteamento explícito com controle de rótulo explícito para um melhor grau de controle

(x) Parâmetros de tráfego específicos por tecnologia

(xi) Manipulação do status administrativo do LSP.

Note que o GMPLS é altamente genérico e possui muitas opções. Somente os blocos (i), (ii) e (x) são obrigatórios, e somente o formato específico é necessário. Tipicamente, os blocos (vi) e (ix) devem ser implementados. Os blocos (iii), (iv), (v), (vii), (viii) e (xi) são opcionais.

Uma rede de comutação SONET/SDH típica implementa os blocos (i) e (ii) (rótulo SONET/SDH), (vi), (ix), (x) e (xi). Os blocos (vii) e (viii) são opcionais, pois proteção/restauração pode ser executada usando bytes de overhead SONET/SDH.

Uma rede típica de comutação por comprimento de onda implementa os blocos (i), (ii) (formato genérico), (iv), (v), (vi), (vii), (viii), (ix) e (xi). O bloco (iii) é necessário somente em caso particular de comutação de faixa de ondas. Uma rede de comutação de fibra típica implementa os blocos (i), (ii) (formato genérico), (vi), (vii), (viii), (ix) e (xi). 
Uma rede MPLS-IP tradicional não implementa nenhum desses blocos pois a ausência do bloco (i) indica MPLS-IP regular. Note, no entanto, que os blocos (ii) e (viii) podem também ser usados para sinalizar MPLS-IP. Neste caso, a rede MPLS-IP pode beneficiar-se do tipo de proteção de enlace (não disponível no CR-LDP e uma forma muito básica disponível no RSVP-TE). Bloco (ii) é aqui um rótulo regular e nenhum formato de rótulo novo é requerido.

\subsubsection{Protocolo de Gerenciamento de Enlace}

O LMP, protocolo de gerenciamento de enlace (no inglês Link Management Protocol), está sendo desenvolvido como parte do conjunto de protocolos GMPLS para gerenciar enlaces TE e permitir que elementos de rede se comuniquem de forma adequada. Este protocolo permite que os nós descubram a identidade e informação detalhada de interfaces dos nós vizinhos e mantém esta informação atualizada assim que configurações ou propriedades dos enlaces TE são modificadas [DATA CONNECTION].

O LMP consiste de quatro funções suportadas pelos controladores de canal de controle e enlace TE; as duas primeiras funções são obrigatórias e as demais são opcionais [DEO, 2003]:

- Gerenciamento de canal de controle

É usado para estabelecer, configurar e manter a conectividade entre nós adjacentes ${ }^{10}$ (mais precisamente, entre controladores GMPLS) através da topologia de rede de controle. Esta operação é realizada utilizando a troca de mensagens de configuração de canal de controle, seguido por uma troca de mensagens do tipo keep-alive.

- Correlação de propriedade de enlace

\footnotetext{
10 Com a separação entre o plano de controle e o plano de dados, vizinhos no plano de controle não necessariamente são vizinhos no plano de dados.
} 
É usada para agregar múltiplos enlaces de dados em um único enlace TE e sincronizar as propriedades local e remota do enlace TE.

- Verificação de enlace

É usada para verificar a conectividade física dos enlaces de dados e o mapeamento entre os identificadores de interface local e remota dos enlaces de dados.

- Gerenciamento de falhas

É usado para suprimir alarmes e localizar falhas de enlace em redes opacas e transparentes.

\subsection{FUNCIONAMENTO}

Considere uma rede GMPLS como mostra a Figura 2.9, que está sob controle do plano de controle GMPLS. O estabelecimento de LSPs entre nós da rede é feito através de uma sucessão de ações, como descrito a seguir. Aqui, a função de cada protocolo no processo de estabelecimento de um LSP é descrita [LI, 2002].

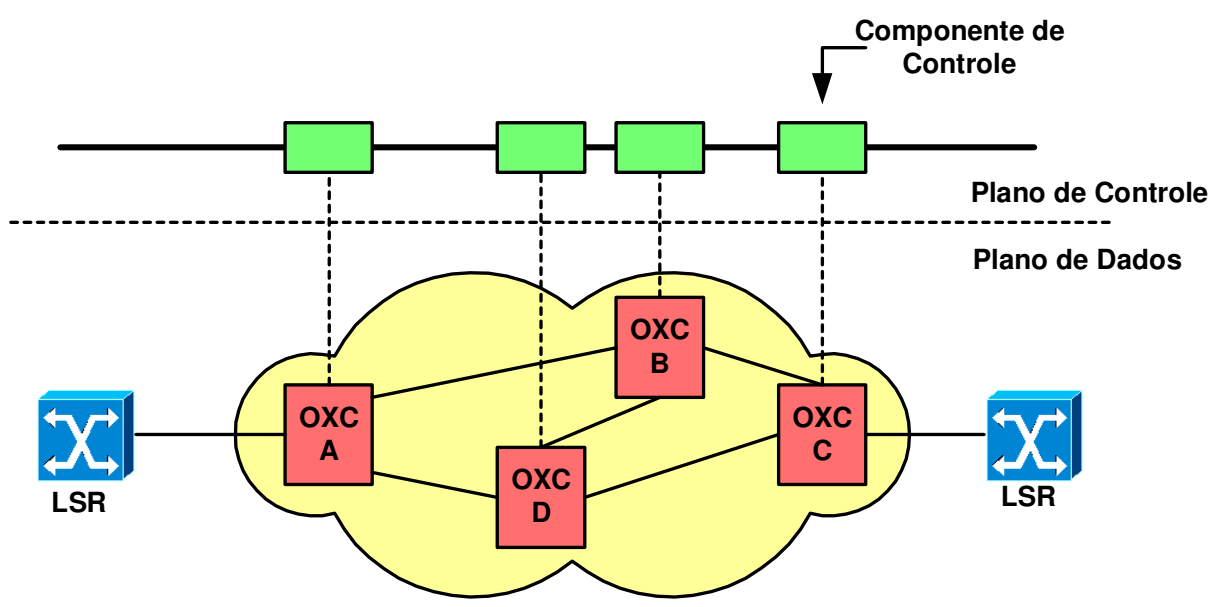

Figura 2.9 - Rede GMPLS. 


\subsubsection{Descobrimento de Vizinhos}

Para qualquer nó na rede requer-se que este conheça outros elementos de rede, as interfaces disponíveis, e topologia dos enlaces entre estas interfaces.

Função do LMP no estabelecimento de enlace

Depois da inicialização de um nó, ele negocia as propriedades de enlace (e.g. formato de dados, monitoramento de erros) com o elemento de rede na outra terminação de cada enlace disponível. Após finalizar tais negociações com sucesso, cada elemento de rede está apto a realizar plena comunicação com seus vizinhos.

Função do OSPF-TE no descobrimento de vizinhos

No próximo passo, o protocolo de descoberta de topologia é iniciado. Primeiro ele aprende quais elementos de rede estão diretamente conectados aos seus enlaces. Por exemplo, o nó A aprende que o nó B e o nó D são seus vizinhos. A seguir, eles trocam todas as informações que receberam com cada um. Assim, nó A sabe que o nó C está a dois hops de distância. OSPF-TE também distribui informação sobre o uso de recursos em cada nó. Esta informação preenche uma base de dados TE que mantém registro da utilização de recursos e desempenho de cada nó na rede. O protocolo de descobrimento é executado continuamente e atualiza a base de dados TE em tempo real. Neste ponto, todos os elementos de rede participantes do plano de controle possuem conhecimento completo da rede. A rede está pronta para aceitar pedidos de serviço. 


\subsubsection{Cálculo de Caminhos}

Para um serviço particular, um caminho apropriado é encontrado através da rede tornando eficiente o uso dos elementos de rede e enlaces.

Função do algoritmo CSPF (Constrained Shortest Path First)

Um pedido de serviço iniciado pelo software de gerenciamento (como SNMP - Simple Network Management Protocol) chega do nó de ingresso. Este nó realiza uma busca em sua base de dados TE para encontrar o melhor caminho entre os pontos finais do serviço. $\mathrm{O}$ melhor caminho é definido como um caminho que minimiza o número de enlaces ou atraso de rede enquanto também satisfaz as restrições e políticas especificadas pelo usuário. As restrições são o aspecto mais interessante do plano de controle inteligente.

Restrições típicas incluem:

- Evitar nós e enlace específicos. Isto é usado com freqüência para obter caminhos independentes de falhas. Nós e enlaces que compartilham os mesmos riscos são coletados em grupos. Os riscos podem ser fibras no mesmo conduto ou, escritórios centrais na mesma zona de terremoto. Logo, pode-se requerer que os caminhos passem por grupos diferentes.

- Incluir nós específicos. Um caso especial é especificar completamente cada nó no caminho. Isto pode ser usado em casos onde o cálculo de caminhos manualmente é desejado.

- Respeitar certas propriedades de atraso e jitter. 


\subsubsection{Sinalização de Serviço}

A configuração de cada elemento de rede do caminho, de acordo com todos os parâmetros necessários, deve ser realizada para que o serviço seja iniciado.

Função do RSVP-TE ou CR-LDP na sinalização de serviço

Assim que um caminho é selecionado, protocolos de sinalização são utilizados para associar cada elemento de rede ao caminho. Em cada nó, o gerenciamento de recurso é realizado para garantir que o atendimento do serviço permita que o novo serviço e todos os serviços já existentes tenham suas QoS garantidas.

Cada nó ao longo do caminho faz uma verificação final e reserva recursos para o serviço. Se qualquer nó não pode cumprir os requisitos do serviço, um erro é gerado e todos os recursos reservados em outros nós no caminho são liberados. Neste ponto, o cálculo do caminho é repetido com base na informação atualizada. Assim que a sinalização de serviço estiver completa, o serviço pode ser liberado e tornar disponível ao usuário final.

O trabalho do plano de controle não pára apenas porque o serviço foi criado. Ele continuamente atualiza a base de dados TE para lidar com falhas e troca de carga na rede.

\subsection{GRUPO DE ENLACES DE RISCO COMPARTILHADO - SRLG}

SRLG, no inglês shared risk link group, fornece parâmetros fundamentais para o gerenciamento de recursos no plano de controle GMPLS e necessários para planejar a confiabilidade nas redes de transporte. Um SRLG está associado com uma entidade em risco, 
tipicamente um segmento de fibra, ou seja, um SRLG define um conjunto de enlaces que compartilham um risco em comum (por exemplo, um recurso físico comum tal como enlace de fibra ou um cabo de fibra) [PAPADIMITRIOU - Draft, 2003]. Um conjunto de enlaces pode constituir um grupo de enlaces de risco compartilhado (SRLG) se estes compartilham um recurso cuja falha pode afetar todos os enlaces no conjunto e, um enlace pode pertencer a múltiplos SRLGs. Assim, a informação SRLG (SRLG-Info) de cada enlace descreve a lista de SRLGs, ou seja, os grupos de risco, aos quais este enlace pertence. Por exemplo, um conjunto de enlaces $L$ pertence ao mesmo SRLG $s$, se eles existirem sobre o mesmo enlace de fibra $f$ [SEBOS, 2002], [STRAND, 2001], [OKI, 2004], [MIYAMURA, 2004].

Uma vez que os nós recebem a informação de enlace TE, as relações SRLG correspondentes podem ser computadas em uma base regular. O conjunto SRLG de cada enlace é propagado uma vez na configuração inicial entre os vários nós utilizando extensões TE do GMPLS para IGPs (OSPF-TE, ISIS-TE). Depois desta troca inicial de identificadores SRLG, valores correspondentes não são alterados com o tempo. No entanto, a propagação de informação SRLG é necessária sempre que um novo enlace é adicionado ou um enlace já existente é removido. A informação SRLG é opcional e se a Propagação de Estado de Enlace (Link State Advertisement - LSA) não transportar a informação SRLG isto significa que o SRLG daquele caminho não é conhecido [PAPADIMITRIOU - Draft, 2003].

Um SRLG é identificado por um número de 32 bits que é único no domínio IGP. A informação SRLG é uma lista não ordenada de SRLGs aos quais o enlace pertence. O SRLG de um LSP é a união dos SRLGs dos enlaces no LSP. O SRLG de um enlace associado é a união dos SRLGs de todos os enlaces componentes. 
O conceito SRLG foi concebido no desenvolvimento de mecanismos de recuperação de falhas. Ele (SRLG) é considerado como um dos critérios mais importantes com relação a comutação de caminhos baseados em restrições nas rotas de canal óptico. Aplicando o critério de disjunção SRLG na computação de caminhos baseados em restrições, pode-se selecionar uma rota levando em consideração a separação de estrutura lógica e de recursos. Isto implica em uma probabilidade mais baixa de falhas de canal óptico simultaneamente. Se são atribuídos diferentes comprimentos de onda, em uma única fibra para um LSP primário e o seu LSP de backup, um simples corte na fibra resultaria na falha de ambos caminhos, LSPs primário e de backup. Para evitar este problema, o conceito SRLG foi introduzido para garantir a disjunção destes dois LSPs.

A Figura 2.10 apresenta uma rede com SRLG, onde o número associado com cada enlace identifica o SRLG [STRAND, 2001]. Na Figura 2.10(a), os enlaces 1-4 e 3-2 possuem o mesmo número SRLG (SRLG \#4), o que significa que estes enlaces ópticos compartilham a mesma fibra como apresentado na Figura 2.10(b). Isto é conhecido como problema de traps (emboscada) nas redes SRLG, ou seja, caminhos/enlaces que aparentemente são distintos, mas fisicamente são o mesmo enlace (um problema importante que diminui o desempenho das redes com relação a probabilidade de bloqueio mas muitos protocolos de roteamento e atribuição de comprimento de onda não consideram) [STRAND, 2001], [SEBOS, 2002], [MIYAMURA, 2004], [PAPADIMITRIOU - Draft, 2003].

No domínio óptico, o conceito SRLG é usado para derivar um caminho, que é disjunto em relação aos recursos lógicos (ou ativos) e físicos (ou passivos). Os requisitos correspondentes quando considera-se uma rede física, topologia e riscos associados podem ser resumidos como segue [MIYAMURA, 2004]: 
- O mecanismo de codificação do SRLG deve reduzir a complexidade de computação de caminho

- A quantidade de informação SRLG deve ser delimitada para reduzir a quantidade de informação que é trocada entre domínios e inter domínio

- A codificação SRLG deve acomodar as restrições lógicas e físicas impostas na diversidade de requisitos

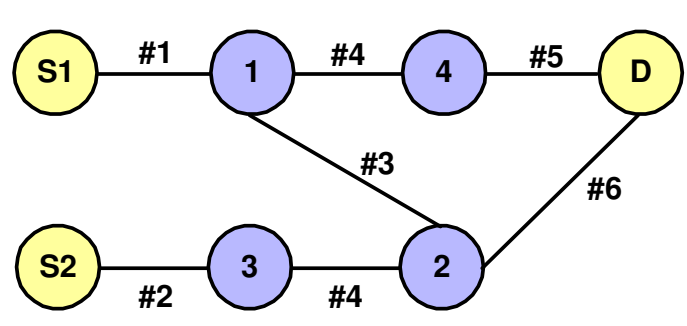

(a) topologia da camada óptica

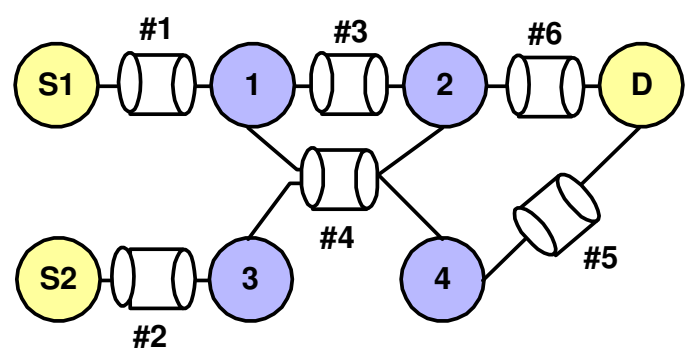

(b) topologia física

Figura 2.10 - SRLG - Exemplo de um modelo de rede.

\subsubsection{Propriedades SRLG}

As propriedades SRLG podem ser resumidas como segue:

- Um enlace pertence a mais de um SRLG se e somente se ele cruza (pelo menos) um dos recursos usados por cada um destes SRLGs.

- Dois enlaces pertencentes ao mesmo SRLG podem pertencer individualmente a (um ou mais) outros SRLGs.

- O conjunto de SRLGs $S$ de um LSP é definido como a união dos SRLG $s$ individuais dos enlaces individuais compondo este LSP. 


\subsubsection{Disjunção SRLG}

Para definir a noção de disjunção SRLG de LSP, primeiro introduz-se a seguinte definição: um LSP (i.e. sequência de enlaces) cobre um SRLG se e somente se ele cruza um dos enlaces pertencentes ao SRLG específico.

A disjunção SRLG de LSP pode ser definida como:

- Dois LSPs são disjuntos com relação a um SRLG $s 1$ se e somente se um deles não cobre este SRLG (eles não cobrem simultaneamente este SRLG $s 1$ ).

- Dois LSPs são disjuntos com relação a um conjunto de SRLG $S$ se e somente se o conjunto de SRLGs que eles individualmente cobrem são completamente disjuntos (os SRLGs comuns entre os conjuntos de SRLGs que eles individualmente cobrem é disjunto de $S$ ).

O impacto na recuperação é notável. A disjunção SRLG é uma condição necessária (mas não suficiente) para garantir a capacidade de sobrevivência da rede. Com relação aos recursos de rede físicos, um par LSP de recuperação deve ter disjunção SRLG no caso de tipo de recuperação dedicada.

\subsection{CONCLUSÕES}

GMPLS evolui a partir do MPLS, via MP $\lambda S$, através da extensão do paradigma label switching de tecnologias de comutação de pacotes/células/frames para tecnologias orientadas a conexão. Embora o paradigma label switching tenha sido originalmente introduzido para melhorar a velocidade de encaminhamento em redes IP (eliminando o tempo gasto na 
associação de prefixo longa), o foco foi direcionado para melhorar a flexibilidade, aprimorar capacidades de QoS e, mais flexíveis e eficientes mecanismos de controle (possível através de um aprimoramento nas capacidades TE). Excluindo completamente a necessidade de reinventar uma nova classe de protocolos de controle para redes de transporte e de definir um espaço de endereçamento óptico dedicado, o GMPLS permite que a maior parte dos softwares originalmente desenvolvidos para aplicações MPLS-TE sejam re-utilizados.

O GMPLS suporta todas as granularidades de comutação: da capacidade de comutação de pacotes até a capacidade de comutação de fibras. Ele não somente re-utiliza os conceitos MPLS (e.g. engenharia de tráfego do MPLS e recuperação), mas também constrói seus próprios protocolos, i.e., extensões foram definidas para protocolos de roteamento (e.g., OSPF-TE) e protocolos de sinalização (e.g., RSVP-TE). LMP faz parte do conjunto de protocolos GMPLS. Este lida com negociações para sinalização, roteamento, gerenciamento de enlace, e engenharia de tráfego. As principais funções suportadas pelo LMP são: gerenciamento de canal de controle, correlação de propriedades de enlace, gerenciamento de falhas, e verificação de conectividade de enlace.

GMPLS faz a ligação entre as redes IP e as redes ópticas fornecendo interoperabilidade e escalabilidade. Ele aprimora o roteamento e escalabilidade fornecendo hierarquia de envio, enlaces não numerados e associação de enlaces.

O aspecto chave da arquitetura GMPLS é a separação do plano de controle e plano de dados, que permite um mais alto desempenho e funcionamento inteligente. Ele também simplifica as redes apresentando um plano de controle comum. Assim, os vários elementos de rede e 
sistemas de software de múltiplos fabricantes podem trabalhar juntos de forma garantida como uma única rede.

O aprimoramento das capacidades de QoS permite um eficiente transporte sobre uma única rede de diferentes classes de serviço $(\mathrm{CoS})$ tais como voz, vídeo e dados com seus requisitos associados em termos de atraso, jitter e disponibilidade. O controle de rede eficiente e flexível, possível via plano de controle GMPLS unificado, permite um mais rápido e simplificado provisionamento de serviços, resultando no aumento de ganhos e diminuição do OPEX (gerenciamento de rede simplificado). Em resumo, GMPLS atende os requisitos das operadoras para qualquer nova tecnologia para aumentar gastos, diminuir capital e/ou despesas operacionais.

GMPLS promete fornecer um mais rápido provisionamento de serviço com custos operacionais mais baixos. Isto é porque ele pode automaticamente descobrir os recursos da rede, oferece ao operador serviços baseados em restrições específicas com certa QoS e pode estabelecer caminhos peer-to-peer com um único protocolo de sinalização. Novos serviços, como largura de banda sob demanda (BoD - Bandwidth on Demand) são possíveis devido a flexibilidade da arquitetura. Devido às várias vantagens e importantes características, o GMPLS será parte integral nas redes de próxima geração (next generation networks). 


\section{CAPÍTULO III}

\section{REDES GMPLS BASEADAS EM WDM - VISÃO GERAL DO RWA}

\subsection{INTRODUÇÃO}

O avanço explosivo da Internet nos últimos anos, comprovado pelo crescimento no número de usuários, e o surgimento de novas aplicações envolvendo voz e vídeo, além das aplicações de dados, têm provocado um aumento considerável na demanda de largura de banda nas redes de transporte que constituem os backbones dos provedores de serviços de telecomunicações. Dados estatísticos justificam este fato: uma chamada telefônica de voz dura em média 3 minutos e os usuários que se conectam a Internet permanecem conectados em média 20 minutos. As conexões à Internet introduzem cerca de seis vezes mais tráfego na rede que as chamadas telefônicas, e o tráfego na Internet duplica-se a cada seis meses (aparentemente esta tendência continuará neste ritmo por mais algum tempo). Com o objetivo de atender, de forma satisfatória, tal demanda de tráfego, tem sido desenvolvida uma nova geração de redes de transporte baseada em uma infra-estrutura óptica inteligente e de alta capacidade. 
As redes ópticas começaram a surgir com o uso intensivo das fibras ópticas a partir dos anos 80. Este tipo de rede é formado por nós interconectados por enlaces de fibra óptica ponto-aponto criando assim, uma determinada topologia.

As redes ópticas, tal como GMPLS (Generalized Multiprotocol Label Switching), podem ser classificadas em opacas ou transparentes. As redes ópticas opacas realizam a comutação de comprimentos de onda no domínio eletrônico. Nesse tipo de rede são necessários conversores OEO (óptico-elétrico-óptico) responsáveis por converter o sinal óptico em sinal elétrico e vice-versa. Conversores OEO têm o inconveniente de inserir atrasos de processamento além de aumentar significativamente o custo dos equipamentos. Nas redes ópticas transparentes, ou redes totalmente ópticas, não existe nenhum armazenamento (buffering) nos nós intermediários da rede e as funções de roteamento, tal como a comutação de comprimentos de onda, são executadas no domínio óptico eliminando os conversores OEO e suas limitações. Desta forma, os dados são levados da origem até o destino no domínio óptico, sem a necessidade de sofrerem qualquer conversão óptico-elétrico-óptico em algum ponto intermediário da rede.

As redes ópticas GMPLS transparentes com multiplexação por divisão de comprimento de onda (Wavelength Division Multiplexing - WDM) são uma das maiores candidatas para as futuras redes de comunicação de grande distância, como detalhado a seguir. As redes GMPLS WDM parcialmente transparentes serão consideradas no restante deste estudo e citadas apenas como redes GMPLS WDM ou GMPLS. Parcialmente transparentes, pois o roteamento é totalmente realizado no domínio óptico no entanto, a sinalização (algoritmo de atribuição de comprimento de onda) utiliza canais opacos para que informações sejam armazenadas nos nós intermediários da rede. 


\subsection{REDES ÓPTICAS WDM}

Inicialmente, as redes ópticas utilizavam o TDM (Time Division Multiplexing) como técnica de multiplexação sub-utilizando assim, a enorme capacidade de transmissão das fibras ópticas. A técnica TDM utiliza apenas um comprimento de onda na fibra e realiza o compartilhamento da fibra dividindo o tempo de sua utilização para cada conexão requerida.

Com o objetivo de melhorar o aproveitamento da capacidade de transmissão das fibras ópticas, as redes ópticas passaram a usar o WDM, uma técnica de multiplexação que divide a fibra óptica em vários canais WDM de diferentes freqüências ópticas, chamados de comprimentos de onda. Múltiplos sinais ópticos de diferentes comprimentos de onda são multiplexados, assim os sinais ópticos são combinados e transmitidos sobre a mesma fibra. Com o WDM é possível o estabelecimento de mais de uma conexão, simultaneamente, através de uma única fibra óptica, mostrando, portanto ser uma técnica promissora para satisfazer as altas demandas de largura de banda sem o enorme investimento necessário para se instalar fibras ópticas adicionais [ASSI, 2001], [CHAUDHURI, 2000], [KIM, 2003].

As redes ópticas WDM, conhecidas por suas altas taxas de transmissão, são formadas por pares de nós origem-destino que se comunicam através de enlaces ópticos (EO), também chamados de segmentos de fibras ou apenas enlaces. Cada enlace é considerado bidirecional e atualmente consiste de um par de enlaces unidirecionais. Um caminho óptico (lightpath) entre duas estações é um sinal óptico que vai de um nó origem a um nó destino, envolvendo um ou mais nós na rede ao longo de uma rota estabelecida, utilizando um comprimento de onda nos enlaces que ligam esses nós (Figura 3.1). Cada comprimento de onda é sempre representado 
por uma cor, logo diferentes comprimentos de onda são representados por cores distintas [KIM, 2003], [RAMASWAMI, 1995].

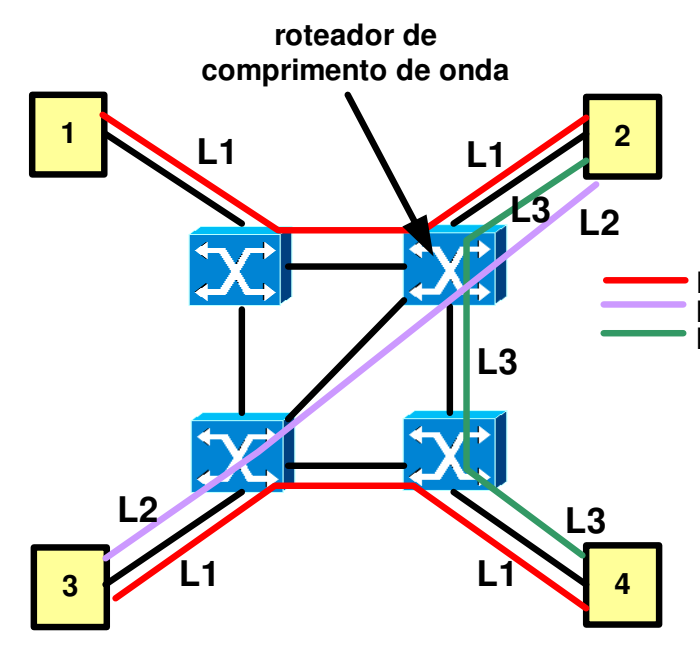

(a) topologia física e caminhos ópticos

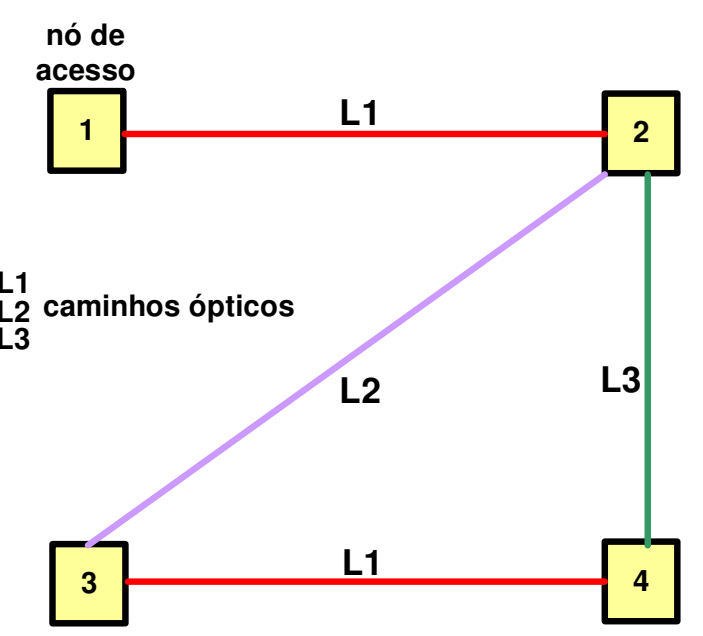

(b) topologia lógica

Figura 3.1 - Rede com roteamento de comprimento de onda.

Através de um caminho óptico uma conexão em uma rede óptica é estabelecida, ou seja, a cada conexão (demanda requerida) na rede é estabelecido um caminho óptico entre o nó origem e o nó destino, utilizando um determinado comprimento de onda. Em geral, seria desejável poder alocar um comprimento de onda diferente para cada pedido de conexão, porém, nas redes ópticas WDM, o número de comprimentos de onda disponíveis nos enlaces limita o número possível de conexões entre cada par de nós. Múltiplos caminhos podem usar o mesmo comprimento de onda, desde que eles não se sobreponham em uma mesma fibra. Esta capacidade espacial de reutilização permite à rede ter um número maior de caminhos utilizando um número limitado de comprimentos de onda. Como apresentado na Figura 3.1, caminhos ópticos entre os nós 1 e 2, 3 e 4 utilizam o mesmo comprimento de onda pois nenhum enlace é compartilhado. Por outro lado, os caminhos ópticos entre 1 e 2,2 e 4, 2 e 3 
devem utilizar comprimentos de onda diferentes pois todas as conexões compartilham o mesmo enlace de fibra.

\subsection{ROTEADOR DE COMPRIMENTO DE ONDA}

A estrutura de um roteador de comprimento de onda é apresentada na Figura 3.2a [WIDJAJA, 2002]. O roteador de comprimento de onda finaliza múltiplas fibras bidirecionais que são usadas para interconectar roteadores de comprimento de onda. Cada fibra de entrada ou de saída tipicamente transporta múltiplos comprimentos de onda.

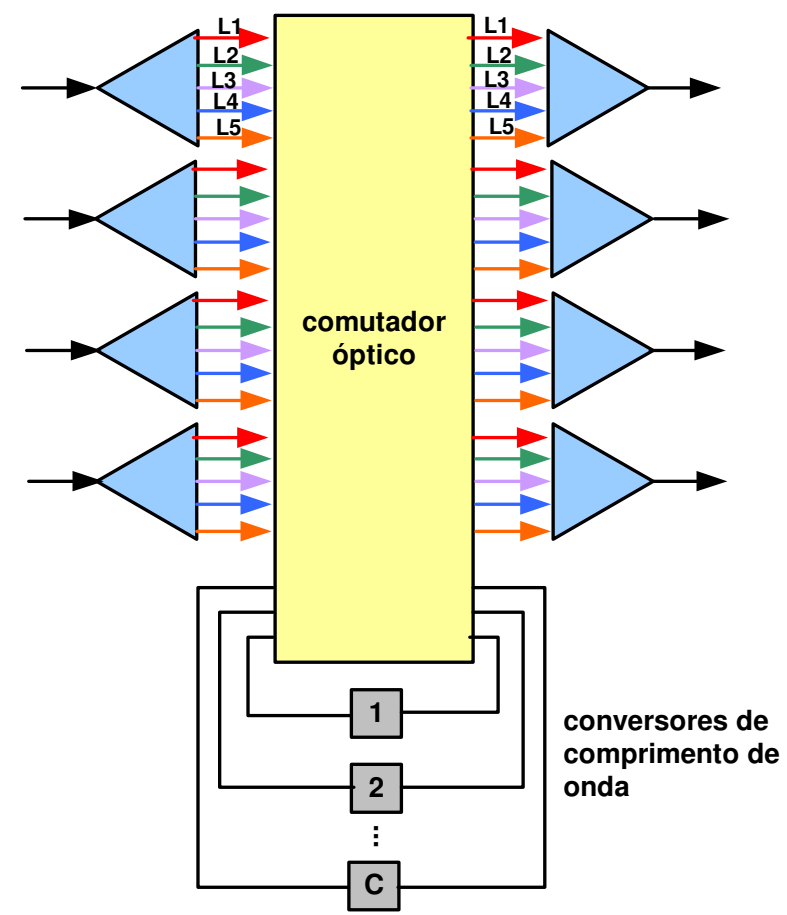

(a) estrutura de um roteador de comprimento de onda

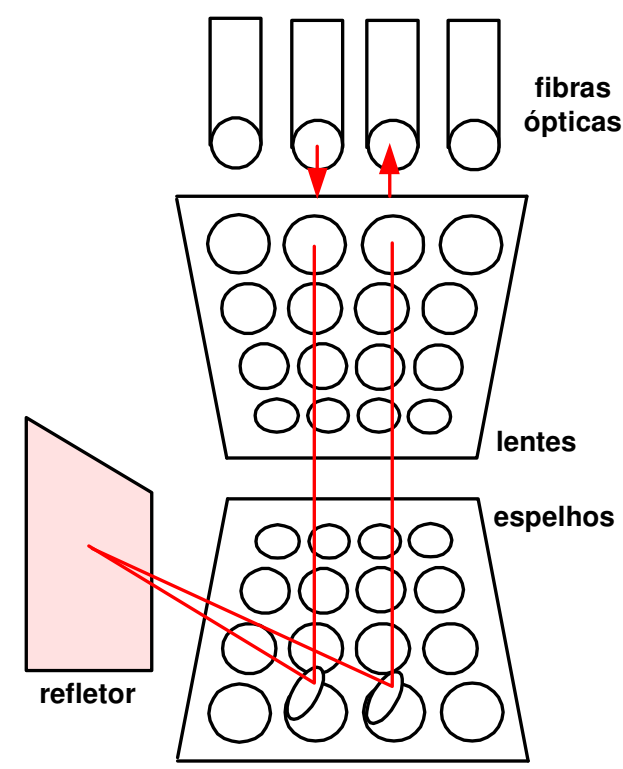

(b) estrutura de um comutador óptico

Figura 3.2 - Roteador de comprimento de onda.

Sinais de entrada de uma fibra são, primeiramente, demultiplexados em comprimentos de onda separados antes de entrar em um comutador óptico (Figura 3.2-a) cuja função é rotear cada comprimento de onda de qualquer entrada para qualquer saída (Figura 3.2-b) através de 
componentes de comutação óptica (MEMS - Micro Electro Mechanical Systems) [KIM, 2003].

Cada conjunto de saídas de um comutador óptico é então multiplexado por comprimento de onda antes de finalmente o sinal ser enviado a uma fibra de saída (Figura 3.2-a). Note que o mesmo comprimento de onda em dois enlaces de entrada não pode ser roteado à mesma fibra de saída simultaneamente. Para evitar colisão de comprimento de onda, o roteador de comprimento de onda pode fornecer um reservatório compartilhado de conversores de comprimento de onda. Quando um comprimento de onda de entrada é para ser roteado para uma fibra de saída que já esteja transportando o mesmo comprimento de onda, o comprimento de onda de entrada precisa primeiro ser roteado para um conversor de comprimento de onda antes de ser re-roteado à fibra de saída (parte inferior da Figura 3.2-a). O conversor de comprimento de onda traduz o comprimento de onda de entrada para um novo comprimento de onda que não esteja em uso na fibra de saída (Figura 3.3).

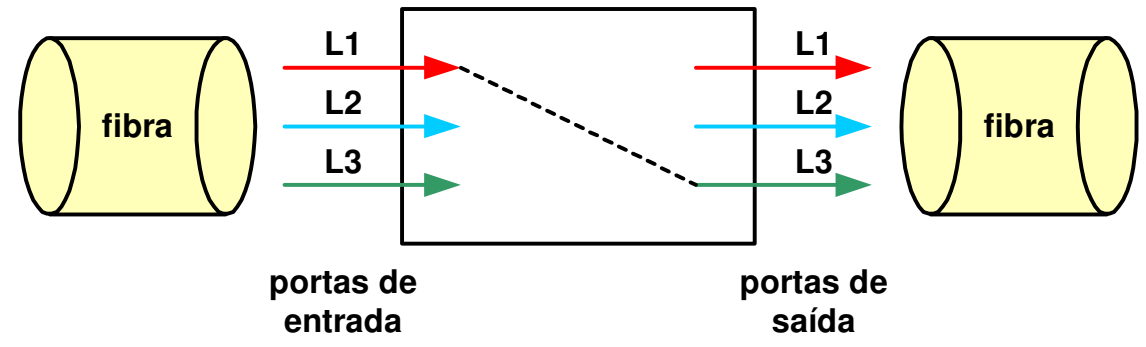

Figura 3.3 - Conversor de comprimento de onda.

Quando um roteador de comprimento de onda não contém conversores de comprimento de onda, este é denominado roteador de comprimento de onda transparente. Um roteador de comprimento de onda que possui um número ilimitado de conversores de comprimento de onda é denominado roteador de comprimento de onda opaco que é similar a um comutador de 
circuito no sentido de que um pedido de entrada pode ser sempre roteado a um enlace de saída se este enlace tiver capacidade disponível suficiente. Um roteador de comprimento de onda que contém um número limitado de conversores de comprimento de onda é denominado roteador de comprimento de onda parcialmente transparente.

\subsection{ENCAMINHAMENTO DE TRÁFEGO}

Dentro das redes ópticas, o tráfego em cada conexão estabelecida pode ser encaminhado pela rede de duas formas: (i) sem conversão de comprimento de onda; (ii) com conversão de comprimento de onda [ZHU, 2000], [CHAUDHURI, 2000], [STRAND, 2001].

\subsubsection{Encaminhamento de tráfego - Sem conversão de comprimentos de onda}

Um caminho óptico usa o mesmo comprimento de onda em todos os enlaces ópticos que o compõem, da origem até o destino. Essa propriedade é chamada de restrição de continuidade de comprimento de onda ou continuidade obrigatória de comprimento de onda. Cada enlace óptico tem um número fixo de comprimentos de onda, disponibilizando vários canais, conforme a tecnologia WDM. Duas conexões que compartilham um enlace comum devem utilizar comprimentos de onda distintos, como mencionado na seção 3.2 [ZANG, 2000], [ROUSKAS, 2001].

A restrição de continuidade de comprimento de onda implica em uma utilização ineficiente dos comprimentos de onda e tem como resultado uma probabilidade de bloqueio de conexões mais alta se comparada com a probabilidade de bloqueio de conexões em redes ópticas com 
conversores. Um pedido de conexão pode ser bloqueado, mesmo que haja comprimentos de onda disponíveis ao longo do caminho que liga os nós origem e destino da conexão solicitada.

A condição de continuidade de comprimento de onda torna a tarefa de atribuição de rota e comprimento de onda mais desafiadora quando comparada aos problemas similares executados nas redes convencionais. A atribuição de rota e comprimento de onda é um problema de controle fundamental.

\subsubsection{Encaminhamento de tráfego - Com conversão de comprimentos de onda}

Diferentes comprimentos de onda podem ser usados pelos enlaces das rotas de conexões ao longo do caminho óptico, ou seja, pode haver conversão de um comprimento de onda para outro comprimento de onda em um mesmo caminho óptico sem, contudo, interferir no sinal de informação que está sendo transmitido. Neste tipo de encaminhamento, é necessária a utilização de conversores nos roteadores para converter o comprimento de onda nos nós intermediários [KARASAN, 1998].

Esses dispositivos permitem uma melhor utilização dos comprimentos de onda, minimizando a probabilidade de bloqueio de conexões, pois pode melhorar a eficiência da rede ao resolver os conflitos de alocação de comprimentos de onda nos caminhos. Porém a tecnologia de conversores de comprimento de onda ainda não está amadurecida e competitiva e, os mesmos possuem um custo muito alto. Deste modo, o foco deste estudo é em redes ópticas que não possuem a capacidade de conversão de comprimento de onda [ZANG, 2000], [ROUSKAS, 2001]. 


\subsubsection{Com conversão x Sem conversão}

Considere $w$ o tamanho do reservatório de comprimentos de onda disponíveis para alocação em cada nó $\left(\lambda_{1}, \lambda_{2}, \ldots, \lambda_{w}\right)$. Caso não haja conversão de comprimento de onda, a rede pode ser analisada como a união de $w$ sub-redes disjuntas, cada qual com um único comprimento de onda (Figura 3.4-a). Quando a rede dispõe de conversão de comprimento de onda, o caminho óptico ao passar por um nó poderá ser transladado para uma outra sub-rede (Figura 3.4-b), desde que: i) o padrão de conversão deste nó permita; ii) a nova sub-rede esteja com seu enlace desocupado.

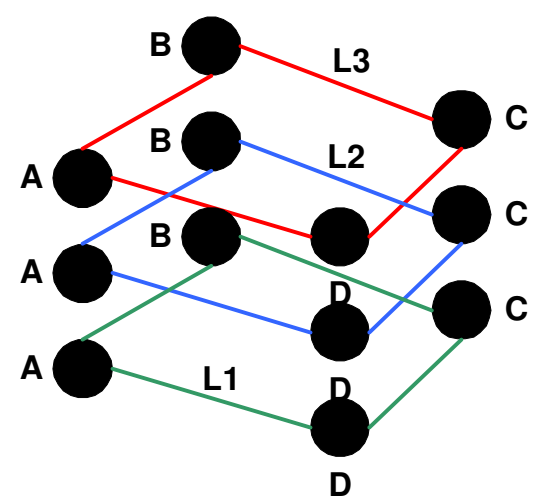

(a) sem conversão

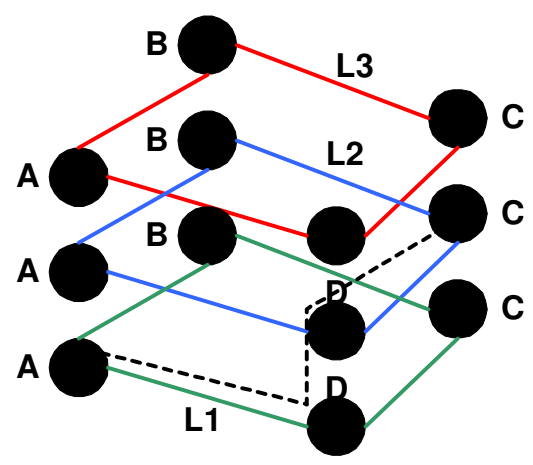

(b) com conversão

Figura 3.4 - Encaminhamento de tráfego.

Devido à limitação na carga das fibras, cada enlace fica fisicamente bloqueado se estiver sendo utilizado em $w$ sub-redes. Dessa maneira, uma requisição por um caminho ou rota é considerada ilegal se um ou mais dos enlaces requisitados estiverem bloqueados. Analisando o comentário anterior e a Figura 3.4b, percebe-se que, se houver convertibilidade plena de comprimento de onda em todos os nós da rede, a condição de continuidade de comprimento de onda desaparece naturalmente e, portanto, apenas requisições ilegais são bloqueadas. Fato este que não ocorre quando não se dispõe de conversão nos nós, pois mesmo uma requisição sendo legal, ela pode ser bloqueada caso não encontre um comprimento de onda que esteja 
disponível simultaneamente em todos os enlaces requisitados, mesmo existindo, em todos eles, comprimentos de onda disponíveis. Para este último caso, entretanto, a escolha de um bom algoritmo de atribuição de rota e comprimento de onda pode reduzir a freqüência dessas ocorrências, ocasionando um melhor desempenho da rede [ROUSKAS, 2001].

\subsection{ROTEAMENTO E ATRIBUIÇÃO DE COMPRIMENTOS DE ONDA}

Um problema muito importante nas redes ópticas WDM é a configuração de caminhos ópticos para um dado conjunto de pedidos de conexões através de roteamento e atribuição de comprimentos de onda para cada uma dessas conexões, dada uma restrição no número de comprimentos de onda disponíveis. Este problema é denominado de problema de roteamento e atribuição de comprimento de onda - RWA (Routing and Wavelength Assignment). O objetivo do RWA é selecionar a melhor combinação de rotas e comprimentos de onda para cada conexão de forma a otimizar o atendimento das demandas dentro da rede, maximizando o número de conexões estabelecidas, minimizando a probabilidade de bloqueio de conexão na rede $^{11}$ e/ou minimizando o número de comprimentos de onda usados pela rede [ZHU, 2000], [ASSI, 2001], [CHAUDHURI, 2000], [STRAND, 2001], [ZANG, 2000], [OKI, 2004].

Os algoritmos RWA da literatura diferem nas considerações de tráfego e nas métricas de desempenho utilizadas. As considerações do tipo de demanda de tráfego, ou seja, o tipo de pedido de conexão, pertencem a uma das categorias mencionadas:

- Demanda estática (ou determinística), na qual um conjunto de pedidos de conexão para pares origem-destino é definida e conhecida a priori.

\footnotetext{
${ }^{11}$ Probabilidade de bloqueio de conexão é definida como a taxa entre o número de conexões bloqueadas e o número total de pedidos de conexão
} 
- Demanda dinâmica (ou aleatória), na qual os pedidos de conexão chegam à rede um a um de forma não conhecida. Em geral, dispõe-se de um conhecimento probabilístico dessa demanda.

Assim, o problema RWA pode ser classificado em:

- RWA estático

No problema RWA estático, todo o conjunto de caminhos ópticos (conjunto de pedidos de conexão) que precisam ser atendidos é conhecido com antecedência e as operações de roteamento e atribuição de comprimentos de onda são feitas offline. $\mathrm{O}$ problema RWA estático se restringe a estabelecer os caminhos ópticos para as conexões de uma maneira global enquanto se minimiza o uso de recursos da rede, como o número de comprimentos de onda ou o número de fibras na rede para uma dada topologia física, de forma a acomodar um determinado número de conexões, ou maximizar o número de conexões acomodadas se o número de comprimentos de onda for limitado. Algoritmos RWA que trabalham de forma estática têm como objetivo planejar a distribuição de demandas dentro da rede. O RWA para a demanda estática é conhecido como problema do Estabelecimento de Caminhos Ópticos Estáticos (Static Lightpath Establishment - SLE) [ZANG, 2000], [ROUSKAS, 2001].

- RWA dinâmico

No problema RWA dinâmico, ou problema de Estabelecimento de Caminhos Ópticos Dinâmicos (Dynamic Lightpath Establishment - DLE), as conexões não são conhecidas previamente, os pedidos de conexão chegam à rede um por vez, de forma aleatória. Um caminho óptico é estabelecido à medida que as requisições de conexões chegam no nó de acesso, devendo cada qual ser atendida no instante da solicitação, 
sem esperar, portanto, que futuras requisições se tornem conhecidas. Os caminhos existentes não podem ser re-roteados para acomodar uma nova requisição, pois isto implicaria uma interrupção momentânea das conexões ativas (o que seria inaceitável principalmente por tratarem-se de taxas de transmissão acima de Gbps), agravado ainda mais pela necessidade de se resolver um problema extremamente complexo de encontrar nova rota e novo comprimento de onda para cada uma das conexões. Qualquer solução do problema de encaminhamento dinâmico deve ser computacionalmente simples, dado que os pedidos de conexão devem ser processados em tempo real. O objetivo do RWA dinâmico é alocar comprimentos de onda tentando minimizar a probabilidade de bloqueio de futuras conexões, dado um conjunto de comprimentos de onda disponíveis, ou seja, maximizar o número de pedidos de conexão atendidos. No caso dos algoritmos RWA que trabalham de forma dinâmica (online) não ocorre uma distribuição planejada de demanda, pois a demanda que chega no nó de origem é tratada individualmente. Os algoritmos dinâmicos têm desempenho pior que os algoritmos RWA estáticos, já que um algoritmo RWA dinâmico não tem conhecimento sobre os pedidos de conexão futuros, enquanto que no caso estático todos os pedidos de conexão são conhecidos com antecedência [MANDAL, 2003], [ZANG, 2001], [TACHIBANA, 2002], [ZANG, 2000], [ROUSKAS, 2001].

Na prática, o problema RWA pode ser encarado de duas formas distintas: alocação conjunta de rota e comprimento de onda ou decomposição do problema em dois subproblemas distintos, subproblema de roteamento e subproblema de atribuição de comprimento de onda:

- Na alocação conjunta de rota e comprimento de onda, uma requisição por um par [rota, comprimento de onda] é gerada. Nessa hipótese, todos os pares disponíveis 
devem ser comparados segundo algum critério a fim de se gerar uma escolha ou alocação.

- Na alocação disjunta de rota e comprimento de onda, cada requisição gera, sucessivamente: (i) uma requisição de rota; (ii) uma requisição de comprimento de onda para a rota requisitada ou bloqueio da mesma.

Do ponto de vista do desempenho da rede, espera-se que a alocação conjunta, caso trate devidamente todas as implicações de se disponibilizar o par [rota, comprimento de onda], apresente melhores resultados, embora seja processualmente de maior complexidade. Ao mesmo tempo, alocações disjuntas de rota e comprimento de onda são mais gerenciáveis, além de provavelmente mais compatíveis com esquemas de proteção. Daí o grande interesse em se abordar a alocação separada de rota e comprimento de onda, comparando-a com a alocação conjunta.

\subsubsection{Subproblema de Roteamento}

O subproblema de roteamento se restringe em encontrar uma rota ou caminho para um par origem-destino de um pedido de conexão. O problema é atualmente tratado com as seguintes técnicas: roteamento fixo, roteamento fixo alternativo e roteamento adaptativo [ZANG, 2001], [MAIER, 2004], [TACHIBANA, 2002], [ZHU, 2000], [ASSI, 2001], [STRAND, 2001], [ZANG, 2000], [ROUSKAS, 2001].

\section{Roteamento Fixo}

No roteamento fixo [GIRARD, 1990] todos os pares de nós origem e destino sempre utilizam uma mesma rota (fixa) estabelecida previamente. Na existência de mais de uma requisição por 
par de nós origem-destino, todas essas requisições disputam o mesmo recurso (conjunto de comprimentos de onda). Isso implica em uma maior probabilidade de bloqueio. Esse tipo de roteamento é muito simples de ser implementado, mas possui desvantagens como:

- Intolerância a falhas nas quais um ou mais enlaces da rede sejam danificados. Para se contornar essas e outras situações, diferentes esquemas de roteamento devem considerar caminhos alternativos ou precisam encontrar uma rota alternativa de forma dinâmica

- Maior probabilidade de bloqueio em decorrência de bloquear todas as conexões que surjam quando não há comprimento de onda disponível na única rota estabelecida, mesmo havendo rotas alternativas que podem atendê-las.

Um exemplo de algoritmo de roteamento fixo é o roteamento de caminho mínimo (Shortest Path Routing - SPR). Nesse algoritmo a rota mais curta para cada par origem-destino é calculada usando algoritmos de caminho mínimo e torna-se a escolhida para que sejam estabelecidas as conexões entre os nós da rede. Três principais algoritmos de caminho mínimo são conhecidos na literatura: Floyd, Bellman-Ford e Dijkstra, com várias aplicações, principalmente em problemas de otimização de rotas de transporte.

\section{Roteamento Fixo Alternativo}

O roteamento fixo alternativo [GIRARD, 1990], [HARAI, 1997], [RAMAMURTHY, 1998] é caracterizado pela existência de mais de uma rota fixa estabelecida previamente. Ao contrário do roteamento fixo, no roteamento fixo alternativo todas as combinações de pares de nós origem-destino possuem mais de uma rota. Para isso, são necessárias tabelas de encaminhamento, ou tabelas de rotas, para armazenar as rotas fixas disponíveis, ordenadas por custo (por exemplo, número de enlaces entre os nós a serem conectados), para um 
determinado nó de destino. Esta tabela pode incluir o caminho mínimo, o segundo caminho mínimo, e assim por diante. Neste caso, o caminho mais curto (menos custoso) é o caminho primário e os demais são considerados alternativos. Esses caminhos podem ser calculados através de algoritmos tais como:

- Algoritmo de Yen - é capaz de determinar os $k$ caminhos mais curtos e que não contém loops, embora esses caminhos não sejam necessariamente disjuntos [YEN, 1972]

- 2-shortest path routing (2-SPR) - esse algoritmo considera apenas as duas rotas mais curtas entre os pares de nós, desprezando assim todas as demais

- Alternative shortest path routing (ASPR) - todas as rotas possíveis entre um nó origem e um nó destino serão disponibilizadas e ordenadas pelo número de enlaces

Quando chega um pedido de conexão nas redes que utilizam roteamento fixo alternativo, o nó origem tenta estabelecer a conexão com o nó destino para cada um dos caminhos da tabela de encaminhamento, de forma seqüencial, começando pelo caminho primário, até que um caminho com comprimento de onda válido (disponível) seja encontrado. Caso não se encontre um caminho disponível na lista de caminhos alternativos, o pedido de conexão é bloqueado e, conseqüentemente, perdido. O roteamento fixo alternativo possui um certo nível de tolerância a falhas e menor probabilidade de bloqueio quando comparado ao roteamento fixo.

Pode-se observar que os algoritmos que empregam o roteamento fixo alternativo, além de não apresentarem uma complexidade de implementação muito elevada, podem ser utilizados para que se disponha de tolerância a falhas nos enlaces dando preferência as rotas que estejam completamente desassociadas com outras rotas, ou seja, não possuam enlaces em comum. 


\section{Roteamento Adaptativo}

No roteamento adaptativo [JUE, 2000], [LI, 1999], o caminho entre um nó origem e um nó destino é escolhido dinamicamente em função do estado corrente da rede. O estado da rede é determinado pelo conhecimento dos caminhos utilizados por todas as conexões atendidas em um instante de tempo. Os algoritmos de roteamento adaptativo são definidos atribuindo-se pesos aos enlaces da rede, os quais, para cada rota, serão somados e comparados a fim de se escolher, mediante métricas específicas, a melhor rota dentre todas as admissíveis. Um exemplo de roteamento adaptativo é o caminho adaptativo de menor custo. Nessa técnica, todos os enlaces ocupados recebem custo $\infty$ (infinito) e os enlaces livres recebem custo 1 . Quando surge uma requisição de caminho óptico de um nó A para um nó B, calcula-se o custo de todas as rotas do nó A para o nó B e a rota utilizada será a de menor custo (soma dos custos dos enlaces que compõem a rota). Se existem múltiplos caminhos com o mesmo menor custo, um deles pode ser escolhido aleatoriamente. Como exemplo de um algoritmo de roteamento adaptativo, existe o Least loaded routing (LLR). Para esse algoritmo são consideradas todas as possíveis rotas entre os pares de nós e a ordenação destas rotas obedece a condição de menor carga na rota. Essa é medida pelas cargas de seu enlace mais congestionado, que por sua vez é dado pelo número de comprimentos de onda que estão sendo utilizados por este enlace. Quando a aplicação do LLR apresenta um conjunto de rotas que fornecem um mesmo valor para a métrica mencionada, este empate pode ser resolvido, por exemplo, empregando-se sobre estas rotas o ASPR.

As vantagens do roteamento adaptativo são: uma maior tolerância às falhas e uma baixa probabilidade de bloqueio quando comparado com o roteamento fixo e com o roteamento fixo alternativo. Por outro lado, o roteamento adaptativo é o mais complexo computacionalmente. 


\subsubsection{Subproblema de Atribuição de Comprimento de Onda}

No subproblema de atribuição de comprimento de onda, deve-se alocar um comprimento de onda para cada caminho óptico de tal forma que dois caminhos ópticos não compartilhem um mesmo comprimento de onda em uma mesma fibra. Dado um conjunto de pedidos de caminhos ópticos e suas rotas e, para otimizar os recursos da rede óptica, a atribuição de um comprimento de onda deve ser feita de forma a maximizar o número de conexões possíveis de serem estabelecidas futuramente minimizando assim a probabilidade de bloqueio de novas conexões.

Duas correntes se mostraram bastantes claras nos estudos heurísticos para atribuição de comprimento de onda: as que provocam uma distribuição igualitária de carga entre todos os comprimentos de onda e as que, contrariamente, sobrecarregam determinados comprimentos de onda em detrimento de outros. Em geral, este último caso utiliza algoritmos que estabelecem algum tipo de prioridade entre os comprimentos de onda, sendo por isso chamados de ajuste prioritário.

É natural se pensar que o fato de se buscar um balanceamento da carga entre todos os comprimentos de onda conduz os algoritmos a apresentarem uma maior capacidade de atendimento. Entretanto, simulações para diversas topologias têm mostrado que, para uma probabilidade de bloqueio fixa, algoritmos que possuem como característica o não balanceamento de carga vêm constantemente apresentando melhores desempenhos, em termos do tráfego escoado, que os de filosofia contrária [ZANG, 2001], [ZHU, 2000], [ASSI, 2001], [STRAND, 2001], [ZANG, 2000], [ROUSKAS, 2001]. 
Os principais algoritmos de atribuição de comprimento de onda propostos na literatura são: First-Fit [CHLAMTAC, 1989], Random Wavelength Assignment [CHLAMTAC, 1989], Most-Used [SUBRAMANIAM, 1987], Least-Used [SUBRAMANIAM, 1987], Max-Sum [BARRY, 1987], Relative Capacity Loss [ZHANG, 1998] e Distributed Relative Capacity Loss [ZANG, 2000]. Como exemplo, os algoritmos serão aplicados no cenário de uma rede óptica transparente em anel unidirecional composta por 6 nós sem conversores de comprimento de onda, e 4 comprimentos de onda $\left(\lambda_{1}, \lambda_{2}, \lambda_{3}, \lambda_{4}\right)$ por enlace, como ilustra a Figura 3.5.

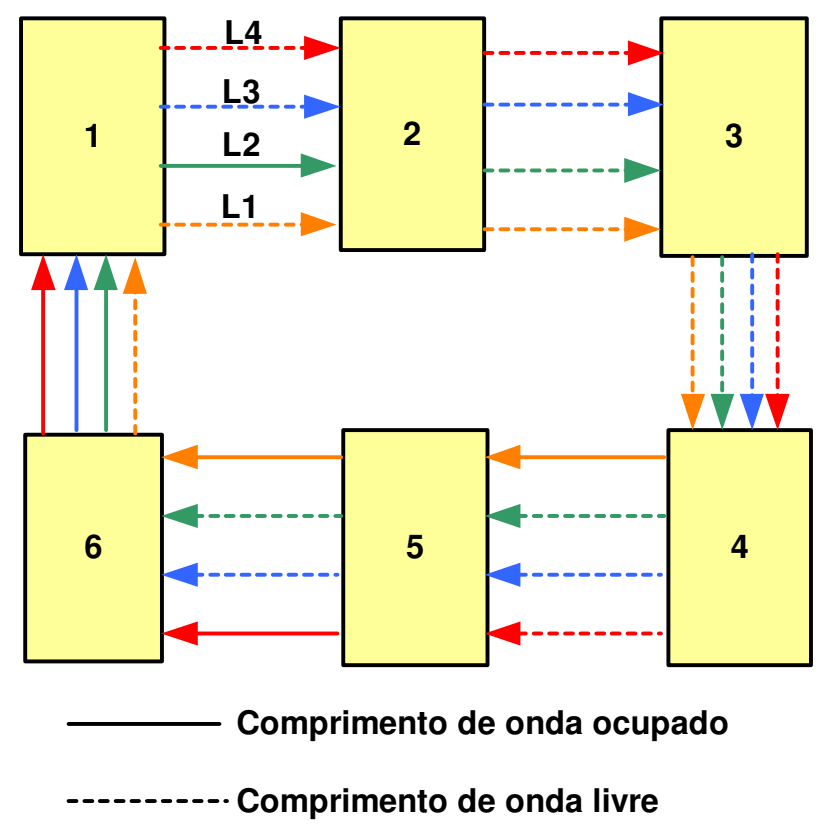

Figura 3.5 - Anel óptico unidirecional com 6 nós e 4 comprimentos de onda.

A notação $(x, y)$ é usada para representar uma conexão ou pedido de conexão com origem no nó $x$ e destino no nó y. O anel óptico possui, no estado inicial, as seguintes conexões estabelecidas: conexão $(4,6)$ utilizando $\lambda_{1}$, conexão $(6,2)$ utilizando $\lambda_{2}$, conexão $(6,1)$ utilizando $\lambda_{3}$ e a conexão $(5,1)$ utilizando $\lambda_{4}$. 
Conhecendo os estados dos enlaces do anel é possível calcular a probabilidade de bloqueio para uma próxima conexão que é dada por:

$\mathrm{Pb}_{\text {prox }}=\mathrm{NCb} / \mathrm{NC}$

Onde:

NCb é o número de conexões bloqueadas dado o estado inicial

NC é o número total de conexões.

$\mathrm{A}^{\mathrm{P}} \mathrm{p}_{\text {prox }}$, no exemplo da Figura 3.5, é igual a 10/30 (as conexões com o mesmo nó de origem e destino são desconsideradas). NC é igual a 30, pois representa o número total de conexões possíveis de serem estabelecidas, ou seja, é a combinação de conexões $(x, y)$ entre todos os nós sendo que $x$ pode variar de 1 a 6 bem como $y$ e, as conexões devem ser consideradas de forma que $x$ e $y$ sejam diferentes (Tabela 3.1).

Tabela 3.1 - Possíveis conexões em uma rede em anel unidirecional com 6 nós.

\begin{tabular}{|l|l|l|l|l|l|}
\hline$(1,2)$ & $(2,1)$ & $(3,1)$ & $(4,1)$ & $(5,1)$ & $(6,1)$ \\
\hline$(1,3)$ & $(2,3)$ & $(3,2)$ & $(4,2)$ & $(5,2)$ & $(6,2)$ \\
\hline$(1,4)$ & $(2,4)$ & $(3,4)$ & $(4,3)$ & $(5,3)$ & $(6,3)$ \\
\hline$(1,5)$ & $(2,5)$ & $(3,5)$ & $(4,5)$ & $(5,4)$ & $(6,4)$ \\
\hline$(1,6)$ & $(2,6)$ & $(3,6)$ & $(4,6)$ & $(5,6)$ & $(6,5)$ \\
\hline
\end{tabular}

NCb é igual a 10 pois dentre todas as possíveis conexões, 10 conexões são bloqueadas. Essas conexões bloqueadas são: $(2,1),(3,1),(3,2),(4,1),(4,2),(4,3),(5,2),(5,3),(5,4),(6,5)$. As conexões $(1,2),(1,3),(1,4),(1,5)$ e $(1,6)$ são possíveis de serem estabelecidas, independente do comprimento de onda a ser utilizado e o mesmo acontece com as demais conexões não bloqueadas. A conexão $(2,1)$ é bloqueada, pois: (i) o comprimento de onda $\lambda_{1}$ não pode ser usado para estabelecer a conexão pois o mesmo está sendo usado nos enlaces $(4,5)$ e $(5,6)$; (ii) 
os comprimentos de onda $\lambda_{2}, \lambda_{3}$ ou $\lambda_{4}$ não podem ser usados para estabelecer a conexão pois os mesmos estão sendo usados no enlace $(6,1)$. Como todos os comprimentos de onda são utilizados, a conexão $(2,1)$ é bloqueada. O mesmo acontece com as demais conexões bloqueadas citadas anteriormente.

Suponha agora que o próximo pedido de conexão é do nó 2 para o nó 4 . O pedido $(2,4)$ pode ser estabelecido com qualquer um dos 4 comprimentos de onda $\left(\lambda_{1}, \lambda_{2}, \lambda_{3}, \lambda_{4}\right)$. A escolha do comprimento de onda é feita pelo algoritmo de alocação de comprimento de onda com o objetivo de minimizar a probabilidade de bloqueio. A Tabela 3.2 mostra a $\mathrm{Pb}_{\text {prox }}$ após o estabelecimento da conexão $(2,4)$ em função do comprimento de onda alocado.

Tabela $3.2-\mathrm{Pb}_{\text {prox }}$ após o estabelecimento da conexão $(2,4)$

\begin{tabular}{|c|c|}
\hline Comprimento de onda & $\mathbf{P b}_{\text {prox }}$ \\
\hline$\lambda_{1}$ & $12 / 30$ \\
\hline$\lambda_{2}$ & $10 / 30$ \\
\hline$\lambda_{3}$ & $11 / 30$ \\
\hline$\lambda_{4}$ & $10 / 30$ \\
\hline
\end{tabular}

O algoritmo Random Wavelength Assignment (RD) [CHLAMTAC, 1989] cria uma lista com todos os comprimentos de onda disponíveis, ao longo da rota determinada, para atender o pedido de caminho óptico. Em seguida o algoritmo RD escolhe um dos comprimentos de onda de maneira aleatória, normalmente usando uma distribuição de probabilidade uniforme. Para atender o pedido $(2,4)$ (Figura 3.5), o RD pode alocar qualquer um dos 4 comprimentos de onda disponíveis, podendo resultar em diferentes probabilidades de bloqueio para a futura conexão. O algoritmo RD é computacionalmente simples, trabalha realizando balanceamento de carga e não necessita de informação global sobre os estados dos enlaces da rede. O balanceamento de carga é alcançado pela descorrelação entre sucessivas escolhas. 
O algoritmo First-Fit (FF) [CHLAMTAC, 1989] enumera todos os comprimentos de onda (1, $2, \ldots, n=4)$. Quando surge um pedido de conexão, o FF aloca o comprimento de onda disponível (livre) com menor índice e para isso precisa apenas conhecer o estado (livre ou ocupado) dos comprimentos de onda dos enlaces que compõem o caminho da conexão a ser estabelecida e não necessita de informação global dos enlaces da rede. O algoritmo FF possui baixo custo computacional, pois não precisa pesquisar o estado de todos os enlaces da rede e pode ser utilizado em qualquer topologia de rede. $\mathrm{O}$ algoritmo FF provoca um desbalanceamento de carga, sobrecarregando os comprimentos de onda de menor índice. Este algoritmo possui um bom desempenho em termos de probabilidade de bloqueio e justiça (fairness) e, na prática, é um dos mais utilizados devido ao seu pequeno overhead (nenhuma informação global é requerida) computacional e baixa complexidade. Na alocação de um comprimento de onda para o estabelecimento do pedido $(2,4)$ no exemplo da Figura 3.5, o FF aloca o $\lambda_{1}$, que é o comprimento de onda disponível de menor índice.

O algoritmo Most-Used (MU) [SUBRAMANIAM, 1997] sempre aloca o comprimento de onda disponível mais utilizado, baseado nas informações do estado atual da rede. É feita uma contagem de quantos enlaces estão sendo utilizados para cada comprimento de onda. Em seguida, o algoritmo MU aloca o comprimento de onda disponível com o maior número de enlaces ocupados. Para estabelecer a conexão $(2,4)$ (Figura 3.5), o MU pode alocar o $\lambda_{1}, \lambda_{2}$ ou $\lambda_{4}$, pois são os comprimentos de onda mais utilizados (2/6). O seu desempenho é um pouco melhor que o desempenho do algoritmo First-Fit e, significativamente melhor que o desempenho do algoritmo Least-Used (descrito a seguir) embora o overhead de comunicação, armazenamento e custo de computação sejam similares ao do algoritmo LU. 
O algoritmo Least-Used (LU) [SUBRAMANIAM, 1997] é o oposto do MU, pois sempre aloca o comprimento de onda disponível menos utilizado, baseado nas informações do estado atual da rede. Procura-se desta forma, balancear a carga entre todos os comprimentos de onda existentes a cada novo pedido que surge. Para estabelecer a conexão $(2,4)$ (Figura 3.5), por exemplo, o LU alocará $\lambda_{3}$ pois este é o comprimento de onda menos utilizado (1/6). O desempenho do LU é menor se comparado ao algoritmo RD devido ao overhead adicional de comunicação requerido (LU utiliza informação global de rede). LU também requer armazenamento adicional e custo computacional e conseqüentemente não é utilizado na prática.

O algoritmo Max-Sum (MS) [BARRY, 1997] tem como objetivo alocar um comprimento de onda de forma a minimizar a perda de capacidade da rede óptica. Isso significa alocar um comprimento de onda $\lambda_{z}$ que minimiza a probabilidade de bloqueio para futuras conexões, $\mathrm{Pb}_{\text {prox }}$, com esse mesmo $\lambda_{\mathrm{z}}$. Ao receber um pedido de caminho óptico, o MS considera todos os caminhos possíveis com exceção do próprio pedido. Em seguida o MS marca para cada comprimento de onda disponível os caminhos que serão bloqueados se o comprimento de onda em questão for alocado. A perda total de capacidade, na verdade, é a soma de todos os caminhos futuramente bloqueados pela alocação do $\lambda_{\mathrm{z}}$. A matriz de tráfego (conjunto de possíveis pedidos de conexão) deve ser conhecida a priori e a rota para cada conexão é préselecionada. Estes requisitos podem ser conseguidos assumindo que a matriz de tráfego seja estável por um período de tempo. Este é um algoritmo difícil e caro de ser implementado em ambientes distribuídos além de requerer roteamento fixo o que diminui drasticamente o desempenho da rede (o mesmo ocorre com o algoritmo RCL, detalhado a seguir). O algoritmo MS aloca o comprimento de onda com menor número de caminhos bloqueados, isto é, o comprimento de onda com menor perda da capacidade total. Para estabelecer a conexão $(2,4)$ 
(Figura 3.5), por exemplo, o MS pode alocar o $\lambda_{2}$ ou o $\lambda_{4}$, pois ambos apresentam menor perda de capacidade de acordo com a Tabela 3.2.

O algoritmo Relative Capacity Loss (RCL) [ZHANG, 1998] é similar ao MS, mas analisa a perda de capacidade relativa. O RCL analisa qual será a perda relativa na alocação de cada um dos comprimentos de onda disponíveis. Na maioria dos casos (cenários), RCL possui melhor desempenho que o MS. No atendimento do pedido (2,4) (Figura 3.5), por exemplo, a perda relativa para a conexão $(1,3)$ será:

- $1 / 3$ para o $\lambda_{1}$, pois o comprimento de onda $\lambda_{1}$ é um dos 3 possíveis comprimentos de onda $\left(\lambda_{1}, \lambda_{3}, \lambda_{4}\right)$ que podem ser usados para estabelecer a conexão $(1,3)$

- 0 para o $\lambda_{2}$, pois o comprimento de onda $\lambda_{2}$ já está sendo usado no enlace $(1,2)$ e fica impossibilitado de ser usado para a conexão $(1,3)$

- $1 / 3$ para o $\lambda_{3}$, pois o comprimento de onda $\lambda_{3}$ é um dos 3 possíveis comprimentos de onda $\left(\lambda_{1}, \lambda_{3}, \lambda_{4}\right)$ que podem ser usados para estabelecer a conexão $(1,3)$

- $1 / 3$ para o $\lambda_{4}$, pois o comprimento de onda $\lambda_{4}$ é um dos 3 possíveis comprimentos de onda $\left(\lambda_{1}, \lambda_{3}, \lambda_{4}\right)$ que podem ser usados para estabelecer a conexão $(1,3)$

Esse cálculo é feito para todas as possíveis conexões com exceção do pedido. Em seguida é feito um somatório das perdas relativas por comprimento de onda. Por fim, é alocado o comprimento de onda de menor perda relativa. Para o pedido $(2,4)$, por exemplo, o RCL alocará o $\lambda_{2}$ ou $\lambda_{4}$, de acordo com a tabela a seguir. 
Tabela 3.3 - Perda relativa por comprimento de onda.

\begin{tabular}{|l|l|l|l|l|}
\hline & $\lambda_{1}$ & $\lambda_{2}$ & $\lambda_{3}$ & $\lambda_{4}$ \\
\hline$(1,2)$ & $1 / 3$ & 0 & $1 / 3$ & $1 / 3$ \\
\hline$(1,3)$ & $1 / 3$ & 0 & $1 / 3$ & $1 / 3$ \\
\hline$(1,4)$ & $1 / 3$ & 0 & $1 / 3$ & $1 / 3$ \\
\hline$(1,5)$ & 0 & 0 & $1 / 2$ & $1 / 2$ \\
\hline$(1,6)$ & 0 & 0 & 1 & 0 \\
\hline$(2,1)$ & 0 & 0 & 0 & 0 \\
\hline$(2,3)$ & $1 / 4$ & $1 / 4$ & $1 / 4$ & $1 / 4$ \\
\hline$(2,5)$ & 0 & $1 / 3$ & $1 / 3$ & $1 / 3$ \\
\hline$(2,6)$ & 0 & $1 / 2$ & $1 / 2$ & 0 \\
\hline$(3,1)$ & 0 & 0 & 0 & 0 \\
\hline$(3,2)$ & 0 & 0 & 0 & 0 \\
\hline$(3,4)$ & $1 / 4$ & $1 / 4$ & $1 / 4$ & $1 / 4$ \\
\hline
\end{tabular}

\begin{tabular}{|l|l|l|l|l|}
\hline & $\lambda_{1}$ & $\lambda_{2}$ & $\lambda_{3}$ & $\lambda_{4}$ \\
\hline$(3,5)$ & 0 & $1 / 3$ & $1 / 3$ & $1 / 3$ \\
\hline$(3,6)$ & 0 & $1 / 2$ & $1 / 2$ & 0 \\
\hline$(4,1)$ & 0 & 0 & 0 & 0 \\
\hline$(4,2)$ & 0 & 0 & 0 & 0 \\
\hline$(4,3)$ & 0 & 0 & 0 & 0 \\
\hline$(4,5)$ & 0 & $1 / 3$ & $1 / 3$ & $1 / 3$ \\
\hline$(4,6)$ & 1 & 0 & 0 & 0 \\
\hline$(5,1)$ & 0 & 0 & 0 & 1 \\
\hline$(5,2)$ & 0 & 0 & 0 & 0 \\
\hline$(5,3)$ & 0 & 0 & 0 & 0 \\
\hline$(5,4)$ & 0 & 0 & 0 & 0 \\
\hline$(5,6)$ & 0 & $1 / 2$ & $1 / 2$ & 0 \\
\hline
\end{tabular}

\begin{tabular}{|l|l|l|l|l|}
\hline & $\lambda_{1}$ & $\lambda_{2}$ & $\lambda_{3}$ & $\lambda_{4}$ \\
\hline$(6,1)$ & 0 & 0 & 1 & 0 \\
\hline$(6,2)$ & 0 & 1 & 0 & 0 \\
\hline$(6,3)$ & 1 & 0 & 0 & 0 \\
\hline$(6,4)$ & 1 & 0 & 0 & 0 \\
\hline$(6,5)$ & 0 & 0 & 0 & 0 \\
\hline & & & & \\
\hline Total & $51 /$ & $48 /$ & $78 /$ & $48 /$ \\
& 12 & 12 & 12 & 12 \\
\hline
\end{tabular}

O algoritmo Distributed Relative Capacity Loss (DRCL) [ZANG, 2000] tem a mesma heurística do RCL, a diferença é que o DRCL realiza os cálculos de maneira distribuída diminuindo o custo de processamento. Cada nó mantém uma tabela RCL e essas informações são trocadas entre os nós. Assim, cada nó pode saber a perda relativa para qualquer destino em qualquer comprimento de onda. O custo computacional é menor do que o do RCL, pois é realizado de forma distribuída e o mesmo pode ser utilizado em ambientes com roteamento adaptativo, ao contrário do MS e RCL.

\subsubsection{Formulação Matemática - atribuição de comprimento de onda}

A formulação que segue considera que já se resolveu o subproblema de roteamento e se empregou a estratégia de roteamento fixo alternativo com uso do algoritmo de Yen [YEN, 1972]. O problema pode ser descrito numa formulação que é mostrada a seguir [ZANG, 2000]:

Entrada do problema:

- Topologia física de rede, descrita como grafo bi-direcional $G=(V, E)$, onde $V$ é o conjunto formado por estações e cross-connects e $E$ é o conjunto de enlaces da rede 
- Conjunto de estações $N$ da rede $(N \subset V)$

- Matriz da demanda $T_{|N| x|N|}$, o qual especifica as requisições de conexão entre as estações. $T_{s, d}$ é o número de requisições de conexão da estação de origem $s$ à estação de destino $d$

- Conjunto de comprimentos de onda $W$ disponíveis em um enlace

- Conjunto de rotas $P_{s, d}$ para cada par origem-destino $(s, d)$. É o resultado obtido pelo algoritmo de Yen

- Matrizes de constantes de pertinência rota-enlace $V_{l}^{s, d, r}$. Note que $l$ é um enlace e $r$ é uma rota. Tais matrizes derivam das rotas obtidas pelo algoritmo de Yen

$$
V_{l}^{s, d, r}=\left\{\begin{array}{c}
1: l \in r, r \in P_{s, d} \\
0: \text { caso contrário }
\end{array}\right\}
$$

Variáveis

- Variável inteira $\sigma_{s, d}^{r}$ indicando o número de caminhos ópticos para o par $(s, d)$ roteado na r-ésima rota

- Variável binária $\Lambda_{c, l}$ indicando o uso do comprimento de onda $c$ no enlace $l$

$$
\Lambda_{c, l}=\left\{\begin{array}{l}
1: \text { se alguma conexão usa o comprimento de onda } c \text { no enlace } l \\
0: \text { caso contrário }
\end{array}\right\}
$$

- Variável binária $\lambda_{c, r}^{s, d}$ indicando o uso de um comprimento de onda em uma dada rota. Note que esta variável é independente do enlace $l$.

$$
\lambda_{c, r}^{s, d}=\left\{\begin{array}{c}
1: \text { se o comprimento de onda } c \text { é usado pela } \mathrm{r} \text { - ésima rota } \\
\text { entre o par origem - destino }(s, d), \text { onde } 1 \leq r \leq\left|P_{s, d}\right| \\
0: \text { caso contrário }
\end{array}\right\}
$$




\section{Restrições}

As seguintes restrições se aplicam ao problema do RWA.

- O número de comprimentos de onda em um enlace não deve exceder sua capacidade:

$$
\sum_{c \in W} \Lambda_{c, l} \leq|W|, \quad l \in E
$$

- O número de comprimentos de onda usados em um enlace é igual ao número de conexões estabelecidas que usam esse mesmo enlace:

$$
\sum_{s \in N} \sum_{d \in N} \sum_{r \in P_{s, d}} V_{l}^{s, d, r} \times \sigma_{s, d}^{r}=\sum_{c \in W} \Lambda_{c, l}, \quad l \in E
$$

- O número de conexões estabelecidas para cada par origem-destino $(s, d)$ não pode exceder a demanda:

$$
\sum_{r \in P_{s, d}} \sigma_{s, d}^{r} \leq T_{s, d}, \quad s, d \in N
$$

- Os comprimentos de onda escolhidos devem corresponder ao tráfego alocado a cada par origem-destino

$$
\sum_{c \in W} \lambda_{c, r}^{s, d}=\sigma_{s, d}^{r}, \quad s, d \in N, \quad r \in P_{s, d}
$$

- Cada comprimento de onda em um enlace é usado por, no máximo, uma conexão:

$$
\sum_{s \in N} \sum_{d \in N} \sum_{r \in P_{s, d}} V_{l}^{s, d, r} \times \lambda_{c, r}^{s, d}=\Lambda_{c, l}, \quad c \in W, \quad l \in E
$$

- Não há necessidade da restrição de continuidade de comprimento de onda, pois isto já está implicitamente implementado pois se fez $\lambda_{c, r}^{s, d}$ independente de $l$.

Função Objetivo - o objetivo é maximizar o número de conexões que podem ser estabelecidas na rede:

$$
Z=\sum_{s \in N} \sum_{d \in N} \sum_{r \in P_{s, d}} \sigma_{s, d}^{r}
$$




\subsection{CONCLUSÃO}

O desenvolvimento de redes ópticas transparentes ou parcialmente transparentes para compor a futura infra-estrutura de transporte dos backbones dos provedores de serviço de telecomunicações é uma tendência mundial. Os recursos básicos dessas redes são caminhos ópticos compostos de comprimentos de onda multiplexados em WDM nas fibras ópticas. Esses recursos devem suportar a oferta de uma gama variada de serviços de telecomunicações com um provisionamento dinâmico sob demanda e otimizado para um volume de tráfego crescente.

Este capítulo apresentou uma visão geral de diversas abordagens que podem ser usadas para rotear e atribuir comprimentos de onda para conexões em redes GMPLS baseadas em WDM. Pode-se perceber que é mais prático dividir o problema RWA em subproblema de roteamento e em subproblema de atribuição de comprimento de onda por assim ser mais gerenciável além de mais compatível com os esquemas de proteção.

O subproblema de roteamento possui as seguintes abordagens: roteamento fixo, roteamento fixo alternativo e roteamento adaptativo. A técnica de roteamento fixo é a mais simples de ser implementada, porém é a técnica que apresenta maior probabilidade de bloqueio de conexão. Por outro lado, a técnica de roteamento adaptativo apresenta a menor taxa de bloqueio, porém é a técnica mais complexa de ser implementada.

O subproblema de atribuição de comprimento de onda possui as seguintes heurísticas: Random Wavelength Assignment, First-fit, Most-used, Least-used, Max-sum, Relative Capacity Loss e Distributed Relative Capacity Loss. Heurísticas mais complexas como Max- 
sum e RCL fornecem uma menor probabilidade de bloqueio de conexão se comparadas com as heurísticas mais simples no entanto, são utilizadas apenas em cenários com RWA estático. A heurística com melhor desempenho, dentre as heurísticas apresentadas, é a DRCL que pode também ser utilizada em ambientes com RWA dinâmico.

Os algoritmos de alocação de comprimento de onda aqui estudados constituem-se em instrumentos valiosos na otimização dos recursos físicos (comprimento de onda), principalmente quando a tecnologia de conversores de comprimento de onda ainda não está amadurecida e competitiva. 


\section{CAPÍTULO IV}

\section{ANÁLISE LÓGICA DE PROTOCOLOS DE COMUNICAÇÃO}

\subsection{INTRODUÇÃO}

Muitos protocolos de comunicação podem ser modelados como uma rede de duas máquinas de estado finitas de comunicação (MEFCs, no inglês, CFSMs - Communicating Finite State Machines) que consiste em um conjunto de máquinas de estado finitas que trocam mensagens sobre canais FIFO (first-in first-out) e unidirecionais. A Figura 4.1 apresenta um modelo simples de protocolo, a comunicação entre as máquinas de estado M e N e o conteúdo de cada canal de comunicação.

É útil e necessário validar os modelos de protocolos de comunicação mostrando assim que a comunicação dos mesmos satisfaz certas propriedades desejadas, verificando, portanto, se a rede está livre de erros de progresso [SCHOOT, 1998], [GOUDA, 1984], [MOURA, 1986]. 


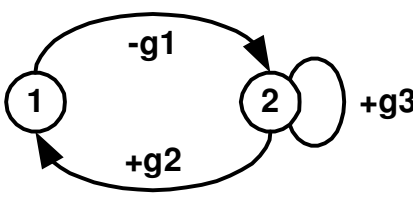

M

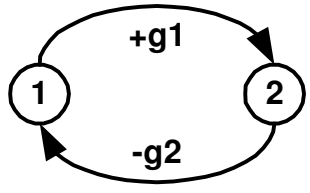

$\mathbf{N}$

Símbolos

- : mensagem enviada

+: mensagem recebida

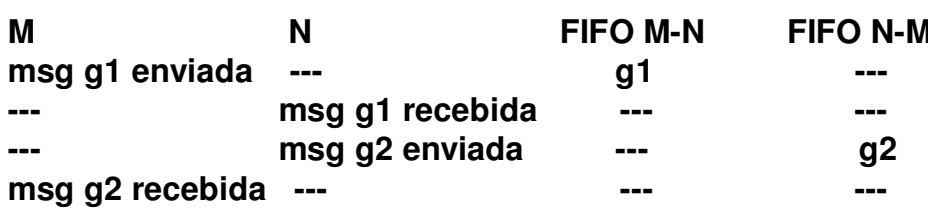

Figura 4.1 - Representação de um Protocolo Simples através de uma Máquina de Estado.

Existem cinco tipos de erros de progresso que um protocolo pode apresentar [YUANG, 1988]. Eles são definidos como:

- Deadlock

Um deadlock é um estado global alcançável a partir do estado global inicial onde todos processos permanecem indefinidamente no mesmo estado, todos os canais estão vazios e nenhuma transição é possível.

- Recepção não-especificada

Uma recepção não-especificada é uma recepção que pode ser executada, mas a mesma não é especificada no projeto do protocolo, portanto o comportamento subseqüente da interação do sistema não é previsível.

- Interação não-executada

Uma interação não-executada é uma transmissão ou recepção que é especificada no projeto do protocolo, mas nunca é executada.

\section{- Livelock}

Um livelock é uma situação onde os processos mantêm a troca de mensagens, mas sem nenhum progresso efetivo sendo realizado. Este erro deve ser evitado, pois assim que o 
mesmo ocorre, o sistema é travado infinitamente em um pequeno conjunto de estados globais.

- Overflow

Um overflow é um estado de canal tal que o número de mensagens no canal não é limitado pelo número inteiro positivo pré-definido. Qualquer canal de comunicação possui uma capacidade de armazenamento que limita a quantidade de informação que o mesmo pode transportar. Uma tentativa em exceder esta capacidade pode resultar em perda de dados sendo transferidos entre os processos envolvidos na comunicação.

A técnica mais direta para validar uma dada rede de duas máquinas de estado finitas de comunicação é chamada exploração de estados e, o método mais comum de análise utilizado por esta técnica é chamado de análise de alcançabilidade exaustiva.

Nesta técnica, constrói-se o grafo de alcançabilidade de uma dada rede; este é um grafo cujos vértices correspondem aos estados alcançáveis da rede e cujos arcos correspondem às transições de estado. Um estado alcançável, também denominado estado global, é uma tupla contendo o estado corrente de cada máquina e o conteúdo de todos os canais utilizados no sistema. Todos os possíveis estados globais são gerados a partir do estado global inicial via transições de saída admissíveis de cada estado em todas as máquinas envolvidas na comunicação [LIN, 1998], [GOUDA, 1984], [NEGULESCU, 1995].

Se o grafo de alcançabilidade for finito, então se determina que a comunicação na rede é limitada e, é possível determinar se a mesma está livre dos erros de progresso, analisando cada estado global novo que é gerado. 
Este modelo tem a vantagem da simplicidade e é um método de análise que pode ser facilmente automatizado. No entanto, este método possui uma grande desvantagem denominada explosão de espaço, apresentada pela maioria das técnicas de verificação existentes; isso é devido ao fato do número total de estados globais que devem ser explorados crescer exponencialmente em função do tamanho da rede [MOURA, 1986].

A maioria dos grafos de alcançabilidade é grande em termos de número de estados globais e a construção dos mesmos requer muito tempo de execução e muita memória podendo tornar impraticável a análise mesmo se o canal for limitado e a análise automatizada. O problema existe, pois o grafo de alcançabilidade exibe o progresso de suas máquinas permitindo todas as velocidades de progresso relativas além de apresentar redundância de exploração de estados na análise de alcançabilidade devido a seqüências equivalentes de transações.

Estas observações fizeram com que Rubin e West sugerissem uma técnica de validação de protocolos melhorada que utiliza um tipo especial de grafo de alcançabilidade chamado grafo de alcançabilidade justa. Nesta técnica, o grafo de alcançabilidade justa de uma rede específica é construído forçando as duas máquinas a avançarem na mesma velocidade. Realmente, o grafo de alcançabilidade justa resultante é normalmente muito menor do que o grafo de alcançabilidade exaustiva convencional correspondente. Rubin e West provaram que se o grafo de alcançabilidade justa é finito, então ele pode ser usado para decidir se a comunicação é livre de deadlocks, recepções não-especificadas, interações não-executadas, liverlocks e overflows.

Para redes de MEFCs com comunicação limitada, que é o caso de praticamente todos os protocolos de comunicação, o método de alcançabilidade justa pode reduzir mais que a 
metade o número total de estados globais que devem ser buscados para verificar erros de progresso. Esta é uma melhora significativa e tem sido utilizada para reparar o problema de explosão de estado. No entanto, o método de alcançabilidade justa pode somente ser aplicado em uma rede de MEFCs com duas máquinas. Esta restrição severamente limita sua aplicabilidade [MOURA, 1986].

\subsection{ESTRATÉGIAS DE REPARAÇÃO EXISTENTES}

As estratégias de reparação apresentadas nesta seção podem ser classificadas em três categorias de acordo com o objetivo de aplicação de cada uma delas. As estratégias na primeira categoria são aquelas de modelagem de protocolo, i.e., no estágio de especificação formal dos protocolos. A segunda categoria de estratégias de reparação é aplicada depois que a modelagem do protocolo é realizada, mas antes que a verificação seja feita. A terceira categoria de estratégias é aquela incorporada na verificação (e assim análise de alcançabilidade) de algoritmos. Uma ênfase maior é dada à terceira categoria de estratégias.

\section{Estratégia de Reparação Proposta por West em 1982 [WEST, 1982]}

A estratégia de reparação proposta por West em 1982 pertence à primeira categoria. As principais técnicas propostas por ele são:

- Restringir o uso de parâmetros multi-valorados tal como número de seqüência na especificação

- Limitar o número de mensagens nos canais de mensagens

- Limitar as classes de erros de transmissão consideradas 


\section{Estratégias de Reparação Propostas por Vuong e Cowan [VUONG, 1982], Choi e Miller} [CHOI, 1983], e Chow, Gouda, e Lam [CHOW, 1985]

Embora diferentes termos tais como decomposição, particionamento e fases múltiplas sejam utilizados entre os grupos de pesquisadores citados, as estratégias de reparação que todos propõem seguem a mesma direção. Eles observaram que certas classes de protocolos podem ser decompostas/particionadas em componentes (ou múltiplas fases) que posteriormente podem ser separadamente verificados para garantir a corretude do protocolo original. Esta técnica soluciona a complexidade do problema de verificação, pois os componentes de protocolos são sempre menores em número de estados e transições do que o protocolo original. Estas propostas são estratégias de reparação da segunda categoria como classificado no início da seção.

\section{Estratégia de Reparação Proposta por Lam e Shankar [LAM, 1984]}

A estratégia proposta aqui também pertence à segunda categoria. Diferentemente das estratégias de decomposição/particionamento, os pesquisadores propõem uma abordagem de projeção, que ao invés de particionar um protocolo em fases múltiplas, constrói um protocolo imagem do protocolo dado para cada uma das funções que se pretende verificar. Os estados, mensagens e eventos das entidades no protocolo imagem são obtidos agregando grupos de estados, mensagens e eventos das entidades correspondentes no protocolo original. $\mathrm{O}$ protocolo resultante, no entanto, é menor do que o protocolo original e, a complexidade do problema é reduzida.

As estratégias de reparação seguintes pertencem todas à terceira categoria, que é a mais importante para este trabalho. 


\section{Estratégia de Reparação Proposta por Blumer e Sidhu [BLUMER, 1986]}

O analisador de máquinas de estado finitas (MEFs), construído como uma das ferramentas no sistema de desenvolvimento de protocolo por Blumer e Sidhu, é baseado no modelo de máquina de estado finita estendida. Um mecanismo chamado de regra de escolha de transição é fornecido, e o mesmo é associado com cada uma das transições. A regra de escolha é uma condição booleana cujo valor decide se a transição na MEFC é ou não para ser executada durante a análise de alcançabilidade. Como exemplo, uma regra pode especificar que nenhuma transição pode ser executada duas vezes no mesmo caminho, ou que nenhuma transição pode ser executada se o resultado é o seu próprio estado, ou loops infinitos que possam ocorrer na análise podem ser eliminados com regras de escolha apropriadas, etc. Como resultado, o escopo da exploração de estados é controlado pelas regras de escolha.

\section{Estratégia de Reparação Proposta por Ansart [ANSART, 1985]}

LISE é também uma ferramenta baseada no modelo de máquina de estado finita estendida. Ela pode ser operada em dois modos: modo de validação e modo de simulação. Quando o sistema é operado no modo de validação, ele pode executar todas as transições possíveis em qualquer estado global. Por outro lado, se o sistema estiver no modo simulação, somente uma transição de saída de um estado global é selecionada para ser executada. O modo simulação é adotado sempre que uma validação completa é inviável devido à explosão de estados. A seleção de transição no modo de simulação é realizada de duas formas. Na primeira, a seleção é simplesmente feita de forma aleatória; na segunda forma, uma prioridade é atribuída para cada uma das transições e a transição com a mais alta prioridade é a escolhida. 
Estratégias de Reparação Propostas por Rubin e West [RUBIN, 1982], Gouda e Han [GOUDA, 1985], Zhao e Bochmann [ZHAO, 1987]

Este grupo de estratégias tem suas bases na exploração de estado com progresso justo. Primeiramente proposta por Rubin e West, e posteriormente estendida por outros pesquisadores, a idéia é explorar somente os estados globais que são alcançáveis, considerando o fato das duas entidades de protocolo avançarem em uma mesma velocidade. Erros de projeto de protocolos tais como deadlock, recepções não-especificadas, interações não-executadas, livelocks e overflow podem ainda ser completamente detectados embora a exploração não seja exaustiva. A limitação desta estratégia é que a mesma é somente aplicada em protocolos de duas entidades.

\section{Estratégia de Reparação Proposta por Gouda e Yu [GOUDA, 1984]}

Esta estratégia é chamada de exploração de estado com progresso máximo. A idéia é similar à exploração de estado com progresso justo e sua aplicabilidade é também limitada a protocolos com duas entidades. Basicamente, a proposta diz que os estados globais de um protocolo com duas entidades podem ser gerados em duas explorações distintas, sendo que durante a execução cada entidade diferente é forçada a progredir em sua velocidade máxima sempre que possível. Todavia, erros de projeto de protocolo tais como deadlocks, recepções nãoespecificadas e sobrecarga de canais, ainda podem ser detectados. Além disso, este método possui outra vantagem sobre os demais que é o fato de que pode ser estruturado para rodar como dois processos de forma a acelerar o processo de validação.

\section{Estratégia de Reparação Proposta por Itoh e Ichikawa [ITOH, 1983]}

A estratégia de reparação proposta por estes pesquisadores é aplicada a protocolos cuja entidade MEFCs não contém qualquer ciclo que não passe pelos estados iniciais. Em cada 
estado global, os eventos admissíveis de diferentes entidades são executados simultaneamente para derivarem o próximo estado global. Além disso, se existe algum evento admissível no estado corrente de uma entidade $E$, derivações adicionais do estado global, através da inibição da execução de todos os eventos admissíveis de $E$, devem também ser realizados. O objetivo é forçar que a entidade $E$ espere com a finalidade de que qualquer um dos potenciais eventos admissíveis possam tornar-se executáveis posteriormente. Seguindo o algoritmo, somente parte do grafo de estados globais é explorada. As seqüências de interação assim exploradas são chamadas de seqüências de exploração reduzidas e são usadas para verificar o protocolo de acordo com a especificação requerida.

Estratégias de Reparação Propostas por Brand e Zfiropulo [BRAND, 1983], Kakuda, Wakahara, e Norigoe [KAKUDA, 1986]

Este grupo de estratégias é chamado de validação de protocolo de árvore (ou forma acíclica). Ao invés de explorar os estados globais de um protocolo, esta estratégia aumenta cada entidade do protocolo em uma árvore ou forma acíclica. Durante o processo de crescimento, erros de projeto de protocolo tais como recepções não-especificadas, deadlocks e sobrecarga de canal podem ser detectados. O algoritmo desta estratégia é muito mais complicado do que a análise de alcançabilidade de estados globais tradicional. Todavia, a velocidade de validação é melhorada.

\section{Estratégia de Reparação Proposta por Holzmann [HOLZMANN, 1985] [HOLZMANN, 1987] [HOLZMANN, 1988]}

Holzmann projetou uma ferramenta chamada Trace, que também trabalha sob dois modos, como ferramenta de depuração rápida ou como um provador de corretude lento. A ênfase principal é que o usuário possa controlar o escopo de cada busca. Quando usado como uma 
ferramenta de depuração, Trace usa uma estratégia de busca chamada busca de espalhamento para explorar o grafo de estados globais, que basicamente é uma busca depth first guiada por alguns heurísticos simples e restrita por um limite de profundidade. Exemplos de heurísticos propostos por Holzmann são:

- Restringir a quantidade de não-determinismo: existem dois tipos de nãodeterminismos em protocolos, aqueles devido à concorrência e outros devido ao comportamento local. É o não-determinismo de concorrência que pode ser removido

- Atribuir prioridades entre os eventos concorrentes: por exemplo, ações internas > ações de recepção > ações de envio > timeout de canal, onde " $>$ " significa "ter prioridade sobre"

- Limitar o tamanho de fila

- Descartar todos os estados depois da exploração depth first exceto aqueles que são estados de loop

- Manter um tamanho limitado de cache para armazenamento de estados globais: descartar estados através de uma seleção simples de round robin quando o cache estiver completo

- Minimizar os modelos MEFCs das entidades de protocolo antes da verificação

\section{Estratégia de Reparação Proposta por West [WEST, 1987] em 1986}

Esta estratégia é chamada de exploração de estados com caminhada aleatória (random-walk).

Da sua experiência em validar o protocolo da camada de sessão do modelo OSI, West observou que a maioria dos erros detectados é encontrada na maior parte das vezes em estados globais diferentes para um protocolo complexo. Isto sugere que a análise de uma subrede de estados globais alcançáveis com caminhada aleatória seja suficiente para identificar uma 
significante fração de erros. A estratégia de caminhada aleatória é então proposta como uma forma de parcialmente explorar o grafo de estados globais. A estratégia diz que:

- Se existe algum evento que pode causar colisão de mensagens quando executado tal evento é executado primeiro; caso contrário, uma escolha arbitrária de qualquer evento a ser escolhido é executada

- A exploração de estados é expandida ao longo de um único caminho sem backtracking. Como resultado, nenhum dos estados anteriores precisa ser lembrado e os estados que já foram explorados podem ser novamente explorados

\section{Estratégia de Reparação Proposta por Vuong, Hui, e Cowan [VUONG, 1982]}

Os pesquisadores propõem uma nova representação de estado global de forma que o algoritmo de alcançabilidade desenvolvido gere grafos finitos para todos os protocolos nãoFIFO e para uma certa classe de protocolos FIFO mesmo que estes protocolos produzam um número limitado de mensagens no meio de transmissão. Esta abordagem então soluciona uma classe de problemas, onde a análise de alcançabilidade convencional falha ao lidar com os mesmos devido a infinidade de estados globais alcançáveis induzidos por acumulação não limitada de mensagens no meio.

Nenhuma das estratégias de reparação pesquisadas anteriormente pode totalmente substituir as outras ou até mesmo a análise de alcançabilidade exaustiva. Por exemplo, embora a validação de protocolo de árvore seja mais rápida do que a análise de alcançabilidade convencional, ela perde a habilidade de detectar propriedades como livelocks e ainda possui um comportamento exponencial no pior caso. Por outro lado, explorações de estado parciais tais como caminhada aleatória, busca de espalhamento, regra de escolha de transição e simulação podem ser capazes de identificar erros de projeto rapidamente, mas não podem 
garantir que o protocolo seja livre de qualquer erro mesmo que nenhum seja encontrado durante a exploração. Finalmente, embora as verificações baseadas no progresso justo ou no progresso máximo e, a especial representação do estado global reduzem a seqüência de implementação e podem completamente validar um protocolo mais rapidamente, elas são aplicáveis somente a algumas classes restritas de protocolos.

A técnica de alcançabilidade justa, ou também denominada de validação com progresso justo, foi a técnica selecionada para verificação dos protocolos GMPLS. A escolha foi realizada com base nas suas principais características (mencionadas anteriormente) tais como: (i) exploração somente dos estados globais alcançáveis, portanto exploração não exaustiva; (ii) validação completa do protocolo mais rapidamente; (iii) diminuição no número de estados no grafo à metade ou menos se comparado com o número de estados pesquisados na análise exaustiva. A desvantagem da técnica de validação com progresso justo que limita o uso da mesma somente em protocolos de duas entidades, não interfere na análise, pois os protocolos GMPLS pertencem a essa classe de protocolos.

\subsection{REDES DE MÁQUINAS DE ESTADO FINITAS DE COMUNICAÇÃ̃O}

Considere $I=\{1,2, \ldots, n\}$, onde $n \geq 2$, é uma constante denotando o número de processos na rede de MEFCs. Uma rede de máquinas de estado finitas de comunicação é uma tupla $\left\langle P_{1}, P_{2}\right.$, $\left.\ldots, P_{n}\right\rangle$, onde cada $P_{i}$ é uma MEFC [GOUDA, 1986], [PARK, 1998], [OZDEMIR, 1994].

Uma máquina de estado finita de comunicação $P_{i}$ é uma tupla $\left(s_{i}, \sum_{i}^{ \pm}, \delta_{i}, p_{0 i}\right)$, onde [GOUDA, 1984] [BRAND, 1983]: 
- $S_{i} \quad$ é o conjunto de estados locais da máquina $P_{i}$.

- $\quad p_{0 i} \in S_{i}$ é o estado inicial da máquina $P_{i}$.

- $\quad \sum_{i}^{ \pm}=\sum_{1 \leq j \leq n} \Sigma_{i, j} \cup \sum_{1 \leq j \leq n} \Sigma_{j, i}$, onde $\Sigma_{i, j}, 1 \leq j, i \leq n$, é o alfabeto de mensagens que $P_{i}$ pode enviar para $P_{j}$, e $\Sigma_{j, i}, 1 \leq j, i \leq n$, é o alfabeto de mensagens que $P_{i}$ pode receber de $P_{j}$.

- $\delta_{i}: S_{i} \times \pm \sum_{i} \times I \rightarrow 2^{S_{i}}$ é a função de transação. $\delta_{i}(p,-m, j)$ é o conjunto de estados que o processo $P_{i}$ pode se mover a partir do estado $p$ depois de enviar uma mensagem $m$ para o processo $P_{j}, \delta_{i}(p,+m, j)$ é o conjunto de estados que o processo $P_{i}$ pode se mover a partir do estado $p$ depois de receber uma mensagem $m$ enviada pelo processo $P_{j}$.

Usa-se $c_{i, j}(i \neq j$, pois se assume que uma máquina não pode enviar mensagens ou receber mensagens dela mesma) para denotar o canal FIFO da máquina $P_{i}$ para $P_{j}$.

Para cada $m \in \Sigma_{i, j},-(m, j)$ denota o evento do processo $P_{i}$ de enviar uma mensagem $m$ para $P_{j}$ (i.e., anexando a mensagem $m$ a $c_{i, j}, \operatorname{logo} c_{i, j} . m$ onde "." é o operador de concatenação), enquanto $+(m, i)$ denota o evento do processo $P_{j}$ de receber uma mensagem $m$ enviada pelo processo $P_{i}$ (i.e., removendo a mensagem $m$ de $c_{i, j}$ ) [LUNDY, 1991].

Uma transmissão $p^{\prime^{-(m, j)}} \rightarrow\left(p^{\prime} \stackrel{+(m, j)}{\rightarrow} p\right.$, resp. $)$ em $P_{i}$ é chamada arco ativo - representa a ação de enviar uma mensagem (arco passivo - representa a ação de receber uma mensagem, respectivamente). Um estado $p$ em $P_{i}$ é denominado estado ativo (estado passivo, respectivamente) se todos os seus arcos de saída são arcos ativos (passivos, respectivamente). $p$ é chamado de estado misto se o mesmo possui arcos ativos e passivos como arcos de saída. 
Um estado global é uma tupla $[\vec{v}, \vec{c}]$, onde $\vec{v}=\left\langle p_{i}\right\rangle_{i \in I}$ são os nós dos processos (MEFCs) envolvidos na comunicação e $\vec{c}=\left\langle c_{i, j}\right\rangle_{i, j \in I}$ são os canais FIFO para troca de mensagens entre os processos. O estado global inicial é $\left[\left\langle p_{0 i}\right\rangle_{i \in I},\left\langle c_{i, j}\right\rangle_{i, j \in I}\right]$, onde $c_{i, j}=\mathcal{E}(i \neq j)$. Um estado global $\left[\left\langle p^{\prime}\right\rangle_{k \in I},\left\langle c_{k, l}^{\prime}\right\rangle_{k, l \in I}\right]$ é subseqüente de um estado global $\left[\left\langle p_{k}\right\rangle_{k \in I},\left\langle c_{k, l}\right\rangle_{k, l \in I}\right]$, se $\exists k, l \in I$ tal que:

- ou $p_{k} \stackrel{-(m, l)}{\rightarrow} p_{k}^{\prime}$ está em $P_{k}, \forall k \in I \quad$ e $c_{k, l}^{\prime}=c_{k, l} \cdot m \quad \forall k, l \in I$

- ou $p_{k} \stackrel{+(m, l)}{\rightarrow} p_{k}^{\prime}$ está em $P_{k}, \forall k \in I$ e $m \cdot c_{l, k}^{\prime}=c_{l, k} \forall k, l \in I$

Embora a noção acima de alcançabilidade por um passo pareça ser complicada, na verdade ela define duas operações simples. No primeiro caso, a máquina $P_{k}$, no estado local $p_{k}$, envia uma mensagem $m$ para a máquina $P_{l}$ e então move para o estado $p^{\prime}{ }_{k}$. Logo somente o canal $c_{k l}$ é alterado. No segundo caso, a máquina $P_{k}$, no estado local $p_{k}$, recebe a mensagem $m$ do canal $c_{l k}$, e então move para outro estado $p^{\prime}{ }_{k}$.

Um estado global $\left[\vec{v}^{\prime}, \vec{c}^{\prime}\right]$ é subseqüente de outro estado global $[\vec{v}, \vec{c}]$, cuja notação é $[\vec{v}, \vec{c}] \stackrel{*}{\Rightarrow}\left[\vec{v}^{\prime}, \vec{c}^{\prime}\right]$, se $\left[\vec{v}^{\prime}, \vec{c}^{\prime}\right]$ é alcançável a partir de $[\vec{v}, \vec{c}]$ em zero ou mais passos. Seja $p$ e $p$ ' dois estados de uma rede $[M, N], p^{\prime}$ é alcançável a partir de $p$ se e somente se $p=p$ ' ou se existem estados $p_{1}, \ldots, p_{r}$ tal que $p=p_{1}, p^{\prime}=p_{r}$ e $p_{i}+1$ é subseqüente de $p_{i}$ para $I=1, \ldots, r-1$.

Um estado de uma rede $[M, N]$ é alcançável se e somente se é alcançável a partir do estado inicial de $[M, N]$.

A tarefa de verificar uma rede de MEFCs que modela um protocolo de comunicação consiste em determinar se a rede de MEFCs possui algumas propriedades não desejáveis que refletem 
em alguns erros lógicos no protocolo original [NEGULESCU, 1995]. Entre as propriedades mais interessantes e investigadas estão deadlocks, recepções não-especificadas, interações não-executadas, livelocks e overflows.

Informalmente, os erros de progresso podem ser detectados da seguinte forma:

- Uma rede de MEFCs está em estado deadlock se cada uma das máquinas estiver em um estado passivo e todos os canais estiverem vazios;

- Uma recepção não-especificada ocorre quando existe um estado global $[\vec{v}, \vec{c}]=\left[p_{1}, p_{2}, c_{12}, c_{21}\right]$ tal que:

(i) $c_{21}=m_{1} \cdot m_{2} \ldots m_{k}(K \geq 1)$ e $p_{1}$ é um no passivo e nenhuma de suas transações de saída estão rotuladas como $+\left(m_{1}, 2\right)$;

(ii) $c_{12}=m_{1} \cdot m_{2} \ldots m_{k}(K \geq 1)$ e $p_{2}$ é um no passivo e nenhuma de suas transações de saída estão rotuladas como $+\left(m_{1}, 1\right)$;

ou seja, quando uma ou mais máquinas na rede estão em um estado local passivo na rede, mas nenhuma das mensagens na primeira posição nos canais FIFO de entrada é compatível com o tipo de mensagem esperada pelos estados passivos locais correntes;

- Interações não-executadas são detectadas quando uma transição de estado está presente na MEFC mas esta está ausente no grafo de alcançabilidade;

- Uma rede de MEFCs apresenta overflow se um dos canais definidos excede o valor limite de mensagens possíveis de serem transmitidas por canal;

- Quando loops infinitos sobre um pequeno conjunto de estados globais é detectado, determina-se então que a rede apresenta livelock, pois transições são realizadas, mas nenhum progresso na rede é constatado. 
Um estado global $[\vec{v}, \vec{c}]$ é estável se $\vec{c}=\vec{c}_{0}$, i.e., todos os canais estão vazios. Aparentemente, um estado deadlock é também um estado estável, mas o contrário não é verdadeiro.

\subsection{ANÁLISE DE ALCANÇABILIDADE JUSTA}

A análise de alcançabilidade justa [GOUDA, 1986] é uma técnica de alcançabilidade melhorada se comparada com a alcançabilidade exaustiva convencional. Esta técnica é utilizada em redes de comunicação com somente dois processos (MEFCs) e, parte do princípio de que os dois processos avançam em igual velocidade. Em outras palavras, ao invés de permitir que somente uma única transição em um processo seja executada por vez, a análise de alcançabilidade justa combina duas transições que se associam entre si de ambos os processos, gerando assim um novo estado global.

Os pesquisadores Rubin e West definiram uma sequiência como uma séria de passos de execução onde cada processo, ou envia, ou recebe uma mensagem. A técnica é baseada na observação de que se cada processo possui uma transição habilitada, então considerar somente um interleaving destas transições é suficiente. Baseado nesta observação, um par de transições, um de cada processo, pode ser executado no mesmo estado global para obter o estado sucessor alcançável para um dos interleavings equivalentes. Como resultado, um par de transições é executado em um vértice do grafo de alcançabilidade. Assim, este método explora todas as seqüências canônicas ao invés de todas as seqüências possíveis.

Um estado $[\vec{v}, \vec{c}]=\left[t, w, c_{t w}, c_{w t}\right]$ da rede $[M, N]$ é justo se e somente se $\left|c_{t w}\right|=\left|c_{w t}\right|$, onde $\left|c_{t w}\right|$ é o número de mensagens no canal de mensagens $c_{t w}$, onde $t$ e $w$ são estados pertencentes as 
MEFCs $M$ e $N$, respectivamente Conseqüentemente, o estado inicial de $[M, N]$ é justo. Portanto, qualquer estado deadlock de $[M, N]$ é também justo.

Um estado global $\left[\vec{v}^{\prime}, \vec{c}^{\prime}\right]$ é, de forma justa, subsequiente de $[\vec{v}, \vec{c}]$ se [PENG, 1997]:

- $\quad\left[\vec{v}^{\prime}, \vec{c}^{\prime}\right]$ é resultado de $[\vec{v}, \vec{c}]$ via duas ações de duas máquinas diferentes, $P_{i}$ e $P_{j}$, tal que a ação de $P_{i}$ seja uma ação ativa (envio) para $P_{j}$, e a ação de $P_{j}$ seja uma ação passiva (recebimento) de $P_{i}$; ou

- $\quad\left[\vec{v}^{\prime}, \vec{c}^{\prime}\right]$ é resultado de $[\vec{v}, \vec{c}]$ via duas ações de duas máquinas diferentes, $P_{i}$ e $P_{j}$, tal que a ação de $P_{j}$ seja uma ação ativa (envio), e a ação de $P_{i}$ seja uma ação passiva (recebimento); ou

- $\left[\vec{v}^{\prime}, \vec{c}^{\prime}\right]$ é resultado de $[\vec{v}, \vec{c}]$ via duas ações de duas máquinas diferentes, $P_{i}$ e $P_{j}$, tal que as ações de $P_{i}$ e de $P_{j}$ sejam ativas; ou

- $\left[\vec{v}^{\prime}, \vec{c}^{\prime}\right]$ é resultado de $[\vec{v}, \vec{c}]$ via duas ações de duas máquinas diferentes, $P_{i}$ e $P_{j}$, tal que as ações de $P_{i}$ e de $P_{j}$ sejam passivas.

Para que as ações passivas mencionadas aconteçam sem a ocorrência de um erro de progresso (recepção não-especificada), os canais envolvidos na comunicação não devem estar vazios e devem conter as mensagens esperadas pelos estados.

Teorema $1^{12}$. Seja $N=\left\langle P_{1}, P_{2}\right\rangle$ uma rede de MEFC. Se um estado global $\left[\vec{v}, \vec{c}_{0}\right]$ é estável, então ele é alcançável de forma justa.

Denomina-se um estado global estável que é alcançável de forma justa como um estado alcançável de forma justa estável. Sabe-se que cada estado alcançável de forma justa é um

\footnotetext{
${ }^{12}$ Não faz parte do escopo desta tese a apresentação da prova do teorema 1 mas a mesma pode ser encontrada em [PENG, 1997].
} 
estado global alcançável válido. Logo, dois corolários imediatos do teorema mencionado são citados, os quais são a base da aplicação do conceito de alcançabilidade justa na detecção dos erros de progresso.

Corolário 1. Uma rede de MEFCs $N$ é livre de deadlocks se e somente se todos os estados alcançáveis de forma justa estáveis em $N$ forem livres de deadlocks.

Corolário 2. Uma rede de MEFCs $N$ é livre de recepções não-especificadas se e somente se todos os estados alcançáveis de forma justa estáveis em $N$ forem livres de recepções nãoespecificadas.

De acordo com o teorema e corolários, para verificar deadlock e recepção não-especificada, precisa-se somente buscar por estados alcançáveis de forma justa estáveis.

Interações não-especificadas é detectada somente com a verificação das transições na rede de MEFCs e os estados globais e canais no grafo de alcançabilidade justa. Por outro lado, livelocks e overflows são detectados a partir da observação do comportamento do grafo de alcançabilidade, verificando a existência de loops infinitos e número de mensagens nos canais, respectivamente.

\section{Grafo de Alcançabilidade Justa}

O resultado final da análise de alcançabilidade justa é a construção do grafo de alcançabilidade justa. Para qualquer rede de MEFCs com comunicação limitada, o número total de estados alcançáveis de forma justa é menor ou igual a metade do número total de estados globais alcançáveis, ou seja, o grafo de alcançabilidade justa é muito menor do que o 
grafo de alcançabilidade exaustiva correspondente. Além disso, o grafo de alcançabilidade justa finito pode ser usado para verificar se um protocolo apresenta ou não erros de progresso.

Os estados alcançáveis de forma justa de uma rede $[M, N]$, e a relação de ser "subseqüente de forma justa" podem ser representados por um grafo rotulado $G$, chamado de grafo de alcançabilidade justa de $[M, N]$, como segue:

- O estado inicial do grafo de alcançabilidade justa é $\left[\vec{v}_{0}, \vec{c}_{0}\right]=\left[t_{0}, w_{0}, \mathcal{\varepsilon}, \varepsilon\right]$, onde $t_{0}$ e $w_{0}$ são os estados iniciais das MEFCs $M$ e $N$, respectivamente e, $\varepsilon$ representa uma string vazia indicando assim que ambos canais unidirecionais não transportam mensagens

- Para cada estado $[\vec{v}, \vec{c}]$ de $[M, N]$ alcançável de forma justa a partir do estado inicial ou de outros estados já presentes em $G$, adiciona-se um vértice rotulado com o estado $[\vec{v}, \vec{c}]$, para $G$ (um vértice rotulado com um estado $[\vec{v}, \vec{c}]$ em $G$ é referido como vértice $[\vec{v}, \vec{c}])$

- Se dois estados alcançáveis de forma justa $\left[\vec{v}^{\prime}, \vec{c}^{\prime}\right]$ e $\left[\vec{v}^{\prime \prime}, \vec{c}^{\prime \prime}\right]$ de $[M, N]$ são de tal forma que $\left[\vec{v}^{\prime \prime}, \vec{c}^{\prime \prime}\right]$ é, de forma justa, subseqüente de $\left[\vec{v}^{\prime}, \vec{c}^{\prime}\right]$ sobre um arco $e$ representando duas ações de transição de duas máquinas diferentes $M$ e $N$, então adicione este arco, rotulado $e$, do vértice $\left[\vec{v}^{\prime}, \vec{c}^{\prime}\right]$ para o vértice $\left[\vec{v}^{\prime \prime}, \vec{c}^{\prime \prime}\right]$ em $\mathrm{G}$

Rubin e West observaram que se o grafo de alcançabilidade justa $G$ (GAJ, no inglês Fair Reachability Graph -FRG) de uma rede $[M, N]$ é finito (i.e. tem um número finito de vértices e arcos), então ele pode ser usado para decidir se a comunicação de $[M, N]$ é livre de deadlocks, recepções não-especificadas, interações não-executadas, livelocks e overflows. Seu algoritmo pode ser como segue:

- A comunicação de $[M, N]$ é livre de deadlock se e somente se nenhum vértice em $G$ for rotulado como um estado de deadlock 
- A comunicação de $[M, N]$ é livre de recepções não-especificadas se e somente se (i) nenhum vértice em $G$ for rotulado como um estado de recepção não-especificada, e (ii) nenhum vértice em $G$ for rotulado como um estado na forma $[t, w, \varepsilon, \varepsilon]$ que é precedente de um estado de recepção não-especificado

- A comunicação de $[M, N]$ é livre de interações não-executadas se e somente se todas as transições definidas nas MEFCs $M$ e $N$ estiverem presente em $G$

- A comunicação de $[M, N]$ é livre de livelocks se e somente se $G$ não possuir nenhum loop infinito em um pequeno conjunto de estados globais

- A comunicação de $[M, N]$ é livre de overflow se e somente se nenhum canal em $G$ exceder o tamanho pré-definido para os mesmos

A terminação deste algoritmo se baseia no fato de $G$ ser finito.

\section{Exemplo}

Considere a rede $[M, N]$ cujas MEFCs $M$ e $N$ são mostradas nas Figuras $4.2 \mathrm{a}$ e $4.2 \mathrm{~b}$ respectivamente e, assuma que cada canal entre as duas máquinas possui capacidade para 3 mensagens. A análise exaustiva da rede $[M, N]$ apresenta 48 estados globais como mostra a Figura $4.2 \mathrm{c}^{13}$. Com o objetivo de diminuir o número de estados globais e ao mesmo tempo verificar completamente os erros de progresso, a análise com progresso justo foi aplicada na mesma rede $[M, N]$. O número total de estados gerados é 10 e o número total de estados distintos é 7. Claramente, esta exploração de estados é mais eficiente. Com base nas definições de erro de progresso, note que a rede é livre de deadlocks, recepções não-especificadas, overflow e interações não-executadas. Mas a mesma apresenta livelocks entre os estados $(3,2,2,4)-(2,3, \mathrm{E}, \mathrm{E})$ e $(2,2, \mathrm{E}, \mathrm{E})-(3,3, \mathrm{E}, \mathrm{E})$.

\footnotetext{
13 A Figura 4.2c foi retirada de [M.G. Gouda, Y. T. Yu, Protocol Validation by Maximal Progress State Exploration, IEEE Transactions on Communications, vol-com- 32, no 1, January 1984]. A figura original apresenta alguns erros que foram corrigidos na Figura 4.2c.
} 


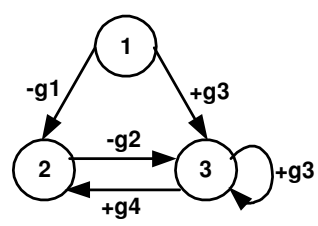

(a)

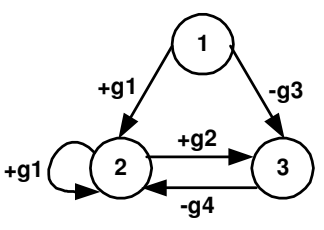

(b)

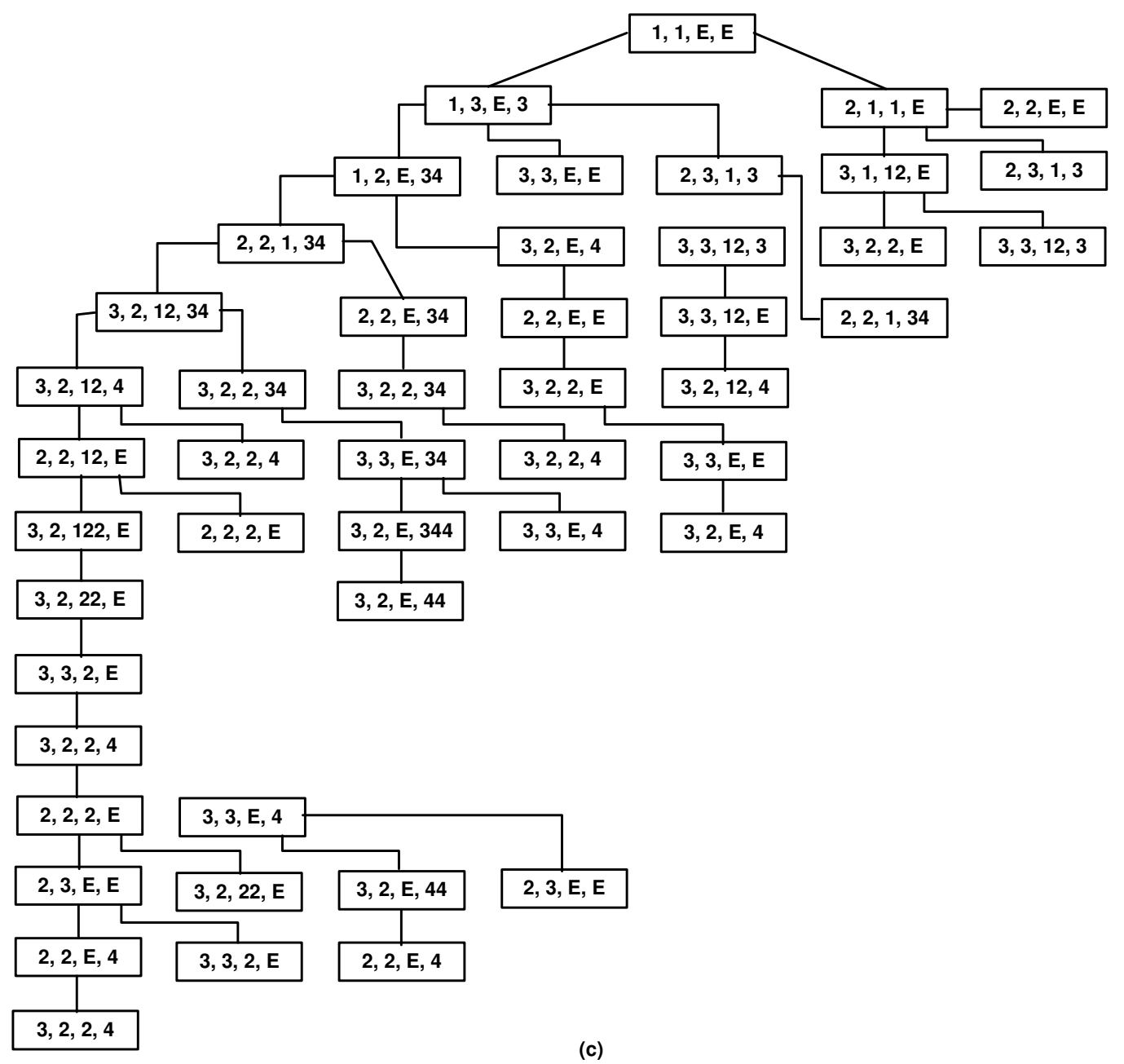




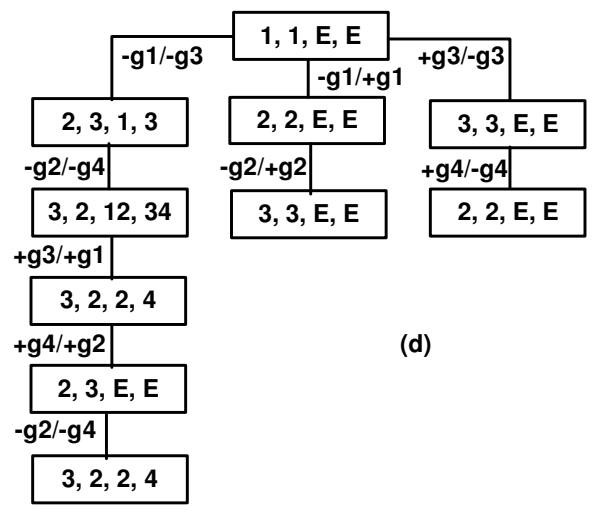

Figura 4.2 - (a) e (b) especificação de dois processos que formam um protocolo através de máquinas de estado finitas; (c) análise de alcançabilidade exaustiva do protocolo; (d) análise de alcançabilidade justa do protocolo especificado.

\subsection{CONCLUSÕES}

Uma rede de máquinas de estado finitas de comunicação é um conjunto de MEF que se comunicam através de um canal FIFO unidirecional enviando e recebendo mensagens. MEFCs tem sido amplamente utilizadas para especificar e principalmente validar protocolos de comunicação.

A técnica mais simples para validar uma dada rede de MEFCs é chamada análise de alcançabilidade exaustiva. Esta explora todas as possíveis interações das MEFCs gerando exaustivamente todos os estados globais alcançáveis a partir do estado inicial, formando assim, o grafo de alcançabilidade da rede. Cada novo estado global é então analisado para determinar se o mesmo representa algum erro de progresso. O processo é repetido para cada estado global gerado até que nenhum estado seja criado. A terminação deste processo é garantida se todos os canais forem limitados e o grafo de alcançabilidade finito.

A análise exaustiva possui uma desvantagem: explosão de estados. Explosão de estados foi e continuará sendo o principal problema das técnicas de verificação de protocolos via análise de 
alcançabilidade. Estratégias de reparação para o problema citado, baseadas em diferentes abordagens, foram pesquisadas e avaliadas.

A análise com progresso justo, uma das técnicas de reparação, foi a técnica escolhida para verificação dos protocolos GMPLS. Nesta técnica, o grafo de alcançabilidade justa de uma rede específica é construído forçando as duas máquinas a avançarem na mesma velocidade. Mostrou-se que a análise justa pode aliviar o problema de explosão, reduzindo à metade ou menos o número de estados globais alcançáveis que devem ser atingidos.

Mesmo com uma exploração não exaustiva dos estados globais, erros de progresso tais como deadlocks, recepções não-especificadas, interações não-executadas, livelocks e overflows, podem ainda ser completamente detectados. A desvantagem desta estratégia é que a mesma é somente aplicada em protocolos de duas entidades, o que não é um limite aos protocolos GMPLS que se encaixam nesta classe de protocolos. 


\section{CAPÍTULO V}

\section{ANÁLISE LÓGICA, PROPOSTA DE CORREÇÕES E MELHORIAS DO PROTOCOLO DE GERENCIAMENTO DE ENLACE}

\subsection{INTRODUÇÃO}

Para permitir a comunicação entre nós vizinhos, garantindo assim o gerenciamento de enlace, roteamento e sinalização, deve existir um par de interfaces IP (Internet Protocol) que são mutuamente alcançáveis. Tal par de interfaces é denominado canal de controle. Note que o termo "mutuamente alcançável" não implica que estas duas interfaces estão diretamente conectadas por um enlace IP; pode existir uma rede IP entre elas. Além disso, a interface sobre a qual as mensagens de controle são enviadas/recebidas pode não ser a mesma interface sobre a qual os dados são transportados.

No GMPLS, não se requer que os canais de controle entre dois nós adjacentes usem o mesmo meio físico que os enlaces de dados entre esses dois nós. Uma das conseqüências em se permitir que o canal de controle entre dois nós seja lógica ou fisicamente diferente e separado 
dos enlaces de dados associados é que a "saúde" do canal de controle não necessariamente correlacione com a "saúde" dos enlaces de dados, e vice-versa.

LMP (Link Management Protocol, no português, protocolo de gerenciamento de enlace) pode comunicar as propriedades dos enlaces ao módulo IGP (Interior Gateway Protocol) que pode assim anunciá-las para outros nós na rede. LMP pode também passar informação do mapeamento entre enlaces TE e canais de controle ao módulo de sinalização. Portanto, LMP realiza uma valiosa função de ligação no plano de controle.

Um protocolo de grande importância, como o LMP, não pode apresentar erros de progresso. Uma análise lógica do protocolo é então realizada através da abordagem de alcançabilidade justa, detalhada no Capítulo IV. Esta análise prova que o LMP está livre de erros de progresso como deadlock, recepção não-especificada, interação não-executada, livelock e overflow.

\subsection{LMP - VISÃO GERAL}

Uma adjacência LMP é formada entre dois nós quando pelo menos um canal de controle bidirecional está estabelecido entre eles. Múltiplos canais de controle podem estar ativos simultaneamente para cada adjacência; no entanto, parâmetros de canais de controle devem ser negociados individualmente para cada canal de controle.

Mensagens LMP são transmitidas de forma confiável usando Message_IDs (identificadores de mensagens) e retransmissões. Somente um Message_ID deve ser incluído em uma mensagem LMP. Para mensagens específicas de canal de controle, o Message_ID está no escopo do canal de controle sobre o qual a mensagem é enviada. Para mensagens específicas 
do enlace TE, o Message_ID está no escopo de uma adjacência LMP. O valor do Message_ID aumenta de forma invariável e automaticamente é zerado quando o valor máximo é alcançado.

Os dois principais procedimentos do LMP são gerenciamento de canal de controle e correlação de propriedades de enlace.

Procedimentos LMP adicionais são definidos: verificação de conectividade de enlace e gerenciamento de falhas. Estes procedimentos são particularmente úteis quando os canais de controle estão fisicamente separados dos canais de dados [ABOUL-MAGD, 2003], [EVERDINGEN, 2002].

\subsection{GERENCIAMENTO DE CANAL DE CONTROLE}

Os canais de controle podem ser usados para troca de informação tais como, provisionamento de enlace e informação de gerenciamento de falhas (implementado usando um protocolo de troca de mensagens como o LMP), gerenciamento de caminho e informação de distribuição de rótulo (implementado utilizando um protocolo de sinalização) e, topologia de rede e informação de distribuição de estado (implementado usando extensões da engenharia de tráfego de protocolos de roteamento) [LANG, 2003].

O gerenciamento de canal de controle (CC) é feito utilizando a troca de mensagem Config e um mecanismo Hello entre os nós. O mecanismo Hello é requerido se os mecanismos de nível mais baixo não estiverem disponíveis para detecção de falhas do canal de controle. Se o mecanismo Hello é usado, mensagens Hello devem ser trocadas sobre o canal de controle. 
Cada nó atribui um identificador de canal de controle (CC_ID) único de 32 bits e diferente de zero a cada direção do canal de controle.

Canais de controle existem independentemente dos enlaces de dados. Múltiplos canais de controle podem estar ativos simultaneamente entre um par de nós e, cada um pode ser implementado de forma diferente. Assim, parâmetros devem ser negociados sobre cada canal de controle individualmente. No caso onde múltiplos canais de controle usam a mesma interface física, a troca de negociação de parâmetros também é realizada para cada canal de controle. As várias mensagens de negociação de parâmetros LMP são associadas com seus canais de controle correspondentes através de seus identificadores únicos (CC-IDs).

Existem quatro mensagens LMP que são usadas para individualmente gerenciar canais de controle. Estas mensagens são: Config, ConfigAck, ConfigNack, e Hello. Elas devem ser transmitidas através dos canais as quais elas se referem. Todas as demais mensagens LMP podem ser transmitidas sobre qualquer canal de controle ativo entre um par de nós adjacentes.

\subsubsection{Negociação de Parâmetros}

A ativação do canal de controle começa com a negociação de parâmetros usando mensagens Config, ConfigAck e ConfigNack. O conteúdo destas mensagens pode ser negociável ou nãonegociável (identificado por um bit $N$ no cabeçalho do objeto). Parâmetros negociáveis podem ser usados para que LMP peers concordem com certos valores. Parâmetros não-negociáveis são usados para anunciar valores específicos que não precisam ou não permitem negociação. 
Para ativar um canal de controle, uma mensagem Config deve ser transmitida a um nó remoto, e como resposta, uma mensagem ConfigAck deve ser recebida pelo nó local.

É possível que ambos, o nó remoto e o nó local, iniciem o procedimento de configuração ao mesmo tempo. Para evitar ambigüidades, o nó com o maior Node_ID (identificador de nó) vence a contenção; o nó com o Node_ID menor deve parar a transmissão da mensagem Config e responder a mensagem Config recebida. Se os Node_IDs são iguais, então um dos nós ou ambos não foram configurados corretamente e, o problema pode ser solucionado por um operador.

A mensagem ConfigAck é usada para reconhecer o recebimento da mensagem Config e expressa a concordância com todos parâmetros configurados (parâmetros negociáveis e nãonegociáveis).

A mensagem ConfigNack é usada para reconhecer o recebimento da mensagem Config e, indicar (caso houver) os parâmetros não-negociáveis que não foram aceitos e propor valores alternativos para os parâmetros negociáveis. Se um nó recebe uma mensagem ConfigNack com valores alternativos não aceitáveis, o nó pode continuar a retransmitir mensagens Config com a configuração considerada correta. O problema pode ser resolvido por um operador mudando os parâmetros em um nó ou em ambos os nós [LANG, 2003].

\subsubsection{Protocolo Hello}

Uma vez que um canal de controle é ativado entre nós adjacentes, o protocolo Hello pode ser usado para manter a conectividade do canal de controle entre os nós e para detectar falhas no 
canal de controle. O protocolo Hello é como um mecanismo keep-alive mais leve que irá reagir rapidamente às falhas do canal de controle de forma que as mensagens Hello não sejam perdidas e as adjacências de estado de enlace associadas não sejam removidas desnecessariamente.

\section{Negociação dos Parâmetros Hello}

Antes de enviar as mensagens Hello, os parâmetros HelloInterval e HelloDeadInterval devem ser determinados e concordados pelos nós local e remoto. Estes parâmetros são trocados na mensagem Config. O HelloInterval indica a freqüência com que as mensagens Hello devem ser enviadas e é medido em milisegundos (ms). O HelloDeadInterval indica quanto tempo um dispositivo deve esperar para receber uma mensagem Hello antes de declarar o canal de controle como desativado e também é medido em milisegundos (ms).

O HelloDeadInterval deve ser maior que o HelloInterval e deve ser pelo menos três vezes o valor de HelloInterval. Se o mecanismo Hello do LMP não for usado, deve-se atribuir o valor zero aos parâmetros HelloInterval e HelloDeadInterval.

Os valores de HelloInterval e HelloDeadInterval devem ser selecionados cuidadosamente para fornecer um rápido tempo de resposta às falhas do canal de controle sem causar congestionamento. Assim, diferentes valores serão configurados para diferentes implementações de canal de controle. Os valores padrões sugeridos para HelloInterval é 150ms e para HelloDeadInterval é 500ms.

Quando um nó transmitir ou receber uma mensagem Config, ele pode começar a enviar mensagens Hello. Uma vez que uma mensagem Hello foi enviada e uma mensagem Hello 
válida foi recebida (i.e., com o número de seqüência esperado), o canal de controle muda para o estado up.

Cada mensagem Hello contém dois números de seqüência: o primeiro número de seqüência (TxSeqNum) é o número de seqüência para a mensagem Hello sendo enviada e o segundo número de seqüência (RcvSeqNum) é o número de seqüência para a última mensagem Hello recebida do nó adjacente sobre este canal de controle. Os números de seqüências nas mensagens Hello permitem que cada nó verifique se o seu peer está recebendo suas mensagens Hello. Através da inclusão do RcvSeqNum nos pacotes Hello, o nó local sabe quais pacotes Hello que o nó remoto recebeu [LANG, 2003].

\subsubsection{Canal de Controle Desativado}

Quando enlaces de dados ainda estão em uso entre um par de nós, um canal de controle pode ser desativado administrativamente se existem outros canais de controle ativos que podem ser usados para gerenciar esses enlaces de dados. Para permitir que um canal de controle seja desativado para finalidades administrativas, a flag ControlChannelDown no cabeçalho comum dos pacotes LMP é utilizada.

Durante este procedimento, um nó deve marcar a flag ControlChannelDown em todas as mensagens LMP enviadas sobre o canal de controle. O nó que inicia o procedimento de desativação do canal de controle pode parar o envio de mensagens Hello depois que HelloDeadInterval milisegundos se passarem, ou se ele receber uma mensagem LMP sobre o mesmo canal de controle com a flag ControlChannelDown marcada. 
Quando um nó recebe um pacote LMP com a flag ControlChannelDown marcada, ele deve enviar uma mensagem Hello com a flag ControlChannelDown marcada e mover o canal de controle para o estado down e o mesmo passa a não ser um canal de controle ativo [LANG, 2003].

\subsubsection{Estado Degradado}

Como conseqüência de se permitir que canais de controle sejam fisicamente separados dos enlaces de dados associados, pode não existir qualquer canal de controle ativo disponível enquanto os enlaces de dados ainda estão em uso. Para muitas aplicações é inaceitável desativar um enlace que está transportando tráfego simplesmente porque o canal de controle não está disponível, no entanto, ao tráfego que está usando os enlaces de dados não será garantido o mesmo nível de serviço. Assim, o enlace TE está no estado degradado e protocolos de roteamento e sinalização devem ser notificados de forma que novas conexões não sejam aceitas [LANG, 2003].

\subsection{CORRELAÇÃO DE PROPRIEDADES DE ENLACE}

A correlação de propriedade de enlace é definida para agregar múltiplos enlaces de dados em um enlace TE e sincronizar as propriedades envolvidas ao enlace TE. Para esta finalidade utiliza-se as mensagens LinkSummary, LinkSummaryAck, e LinkSummaryNack. O conteúdo dessas mensagens é construído usando parâmetros que podem ser negociáveis ou nãonegociáveis. Este procedimento deve ser realizado antes do enlace TE ser estabelecido e colocado em funcionamento bem como, a qualquer momento que o enlace TE esteja ativo, mas não durante o processo de verificação. 
Cada enlace TE tem um identificador (Link_ID) que é atribuído para cada terminação de um enlace. Similarmente, é atribuído um identificador (Interface_ID) para cada enlace de dados em cada terminação.

A mensagem LinkSummary é usada para verificar a consistência das informações dos enlaces de dados e enlace TE em ambos os lados como também, para agregar múltiplos enlaces de dados em um enlace TE e, para sinalizar concordância no mapeamento de Interface_IDs e definições de propriedades de enlace. Logo, a mensagem LinkSummary inclui Link_IDs remoto/local do enlace TE e indica suporte aos procedimentos de gerenciamento de falhas e verificação de enlace para o enlace TE, bem como inclui Interface_IDs local/remoto de todos os enlaces de dados que compõem o enlace TE e, propriedades de enlace.

Por outro lado, uma mensagem LinkSummaryNack deve ser transmitida indicando qual mapeamento de interface não está correto e/ou quais propriedades de enlace não são aceitas. Se uma mensagem LinkSummaryNack é recebida e inclui parâmetros negociáveis, então o iniciador da mensagem LinkSummary deve enviar uma nova mensagem LinkSummary que deve incluir novos valores para os parâmetros negociáveis. Deve-se considerar os valores aceitos que foram recebidos na mensagem LinkSummaryNack [LANG, 2003].

O Internet Draft [draft-ietf-ccamp-lmp-10.txt] menciona que a função de correlação de propriedade de enlace deve ser acionada antes do enlace TE ser estabelecido e colocado em funcionamento e pode ser feita a qualquer momento que o enlace TE esteja ativo, mas não durante processo de verificação. Considerando o primeiro caso, se o enlace TE ainda não foi estabelecido então o nó local tem conhecimento do Link_ID local (identificador de enlace TE local) mas não tem conhecimento do Link_ID remoto. Note que de acordo com a descrição da 
mensagem LinkSummary, esta possui o valor do Link_ID remoto como um dos parâmetros que a compõe, mas nenhuma citação no Internet Draft é feita com relação ao valor que se deve atribuir ao Link_ID remoto caso este não for conhecido. Portanto, deve-se permitir que o Link_ID remoto não seja incluído na mensagem LinkSummary ou que seja incluído com o valor zero e o mesmo seja então aprendido dinamicamente quando o nó local receber a mensagem LinkSummaryAck/LinkSummaryNack como resposta do nó remoto contendo o Link_ID remoto.

Para que isso aconteça com sucesso, já deve ser de conhecimento do nó local todos os Interface-IDs local/remoto dos enlaces de dados que compõe o enlace TE para que não haja erros quanto ao enlace TE sendo utilizado. O conhecimento dos Interface_IDs somente é possível através de uma configuração manual ou, dinamicamente através da verificação de conectividade de enlace que deve portanto, ser obrigatoriamente realizada antes da correlação de propriedade de enlace e assim, deixa de ser um procedimento opcional.

Agregar múltiplos enlaces de dados em um enlace TE é parte das responsabilidades do procedimento de correlação de propriedades de enlace. Os enlaces de dados a serem agregados devem ser indicados em uma lista presente na mensagem LinkSummary que inclui dentre outros parâmetros, os Interface_IDs local e remoto. Estes identificadores, portanto, devem ser conhecidos pelo nó local e isso só é possível através de uma configuração manual ou através do procedimento de verificação de conectividade de enlace. Conseqüentemente, este último procedimento citado, deve ser obrigatoriamente realizado (e não opcional como mencionado por [LANG, 2003]) e antes mesmo do procedimento de correlação de propriedade de enlace. 


\subsection{VERIFICAÇÃO DE CONECTIVIDADE DE ENLACE}

A verificação de conectividade de enlace pode ser usada para examinar a conectividade física de todos os enlace de dados, individualmente para cada enlace de dados, e dinamicamente descobrir as associações dos Interface_IDs local e remoto e de enlace TE.

O procedimento é efetivado somente quando há pelo menos um canal de controle ativo entre os dois nós envolvidos e, deve ser realizado primeiramente para os enlaces de dados que compõem o(s) enlace(s) TE (com pelo menos um enlace de dados ativo) já estabelecido(s), e subseqüentemente, de forma periódica para todos os enlaces de dados não alocados a um enlace TE. Suporte a este procedimento é indicado marcando a flag de suporte à verificação de enlace na mensagem LinkSummary.

Uma característica única de dispositivos transparentes é que os dados não são modificados ou examinados em operação normal. Esta característica é um desafio para validação da conectividade dos enlaces de dados e estabelecimento de mapeamentos de rótulos. No entanto, para garantir uma correta verificação de conectividade de enlace de dados, requer-se que até que os enlaces de dados sejam alocados para tráfego de usuário, eles sejam opacos. Para suportar vários níveis de opacidade e, conseqüentemente diferentes mecanismos para transportar as mensagens Test, um campo de verificação de mecanismo de transporte é incluído nas mensagens BeginVerify e BeginVerifyAck. Como a troca de mensagem BeginVerify coordena o procedimento de Test, ela também naturalmente coordena a transição dos enlaces de dados pra dentro ou fora do modo transparente [LANG, 2003]. 
Quando o canal de controle entre dois nós é estabelecido, o procedimento de verificação de conectividade de enlace pode ser iniciado. Para começar o procedimento de verificação de enlace, o nó local deve enviar uma mensagem BeginVerify através do canal de controle. Se o nó remoto receber uma mensagem BeginVerify e estiver pronto para processar mensagens Test, ele deve enviar uma mensagem BeginVerifyAck com resposta ao nó local especificando o mecanismo de transporte desejado para as mensagens Test. Quando o nó local recebe uma mensagem BeginVerifyAck do nó remoto, ela pode começar a testar os enlaces de dados transmitindo periodicamente mensagens Test.

Uma mensagem Test é transmitida sobre os enlaces de dados sendo verificados e mensagens TestStatus são recebidas como resposta sobre o canal de controle. Note que a mensagem Test é a única mensagem LMP que dever ser transmitida utilizando o enlace de dados. Enlaces de dados são testados na direção de transmissão, pois os mesmos são unidirecionais, e desta forma, pode ser possível para ambos os nós trocarem mensagens Test independente e simultaneamente.

Quando a mensagem Test é recebida, uma mensagem TestSatusSuccess deve ser enviada como resposta. O recebimento de uma mensagem TestStatusSuccess indica que a mensagem Test foi detectada no nó remoto e a conectividade física do enlace de dados foi verificada. Assim, o nó local deve marcar o enlace de dados como up e enviar uma mensagem TestStatusAck ao nó remoto. Se, no entanto, a mensagem Test não for detectada no nó remoto durante um período de observação (especificado pelo VerifyDeadInterval), o nó remoto deve enviar uma mensagem TestStatusFailure sobre o canal de controle indicando que a verificação da conectividade física do enlace de dados falhou. Quando o nó local recebe uma 
mensagem TestStatusFailure, ele deve marcar o enlace de dados como failed e enviar uma mensagem TestStatusAck ao nó remoto.

Assim que todos os enlaces de dados contidos na lista transportada na mensagem BeginVerify forem testados, o nó local envia uma mensagem EndVerify para indicar que o teste está completo entre os dois nós. É também permitido que o transmissor termine o procedimento Test a qualquer momento após o envio da mensagem BeginVerify. A mensagem EndVerify é utilizada para esta finalidade.

\subsection{GERENCIAMENTO DE FALHAS}

Nesta seção, um procedimento LMP opcional é descrito. Este é usado para gerenciar falhas através da rápida notificação do status de um ou mais canais de dados em um enlace TE. O escopo deste procedimento é de um enlace TE, e como tal, o seu uso é negociado como parte da troca da mensagem LinkSummary.

O gerenciamento de falhas utiliza as seguintes mensagens: ChannelStatus, ChannelStatusAck, ChannelStatusRequest,e ChannelStatusResponse. A mensagem ChannelStatus é enviada sem ser solicitada e é usada para notificar um vizinho LMP sobre o status de um ou mais canais de dados de um enlace TE. A mensagem ChannelStatusAck é usada para reconhecer a chegada da mensagem ChannelStatus. A mensagem ChannelStatusRequest é usada para consultar um vizinho LMP sobre o status de um ou mais canais de dados de um enlace TE. A mensagem ChannelStatusResponse é usada para reconhecer a chegada de uma mensagem ChannelStatusRequest e indicar os estados dos enlaces de dados consultados. 
Lembre-se que um enlace TE conectando dois nós pode ser composto por um número de enlaces de dados. Se um ou mais enlaces de dados falham entre os dois nós, um mecanismo deve ser usado para rapidamente notificar a falha para que mecanismos de proteção/restauração apropriados possam ser iniciados. Se a falha é subseqüentemente tratada, então um mecanismo é usado para notificar que a falha já foi tratada e o status do canal é $O K$.

\section{Detecção de Falhas}

Detecção de falhas deve ser manipulada pela camada mais próxima da falha; para redes ópticas, a camada seria a camada física (óptica). Uma medida de detecção de falha na camada física é detectar perda de luz (LoL - Loss of Light). Outras técnicas para monitoramento de sinal óptico estão ainda sendo desenvolvidas. No entanto, deve estar claro que o mecanismo usado para notificação de falhas no LMP é independente do mecanismo usado para detecção de falhas, e simplesmente se baseia no fato de que uma falha é detectada [BRADFORD, 2003].

\section{Procedimento de Localização de Falhas}

Em algumas situações, uma falha no enlace de dados entre dois nós é propagada downstream assim, todos os nós downstream detectam a falha sem localizar esta. Para evitar múltiplos alarmes a partir da mesma falha, LMP fornece a função de notificação de falhas através da mensagem ChannelStatus. Esta mensagem pode ser usada para indicar que um único canal de dados falhou, que múltiplos canais de dados falharam, ou que um enlace TE inteiro falhou.

A correlação de falhas é feita localmente em cada nó a partir do recebimento de uma notificação de falha. 
Para localizar uma falha em um enlace particular entre nós adjacentes, um nó downstream (downstream em termos do fluxo de dados) que detecta a falha no enlace de dados envia uma mensagem ChannelStatus ao seu vizinho upstream indicando que a falha foi detectada (associando a notificação de todos os enlaces de dados com falha). Um nó upstream que recebe a mensagem ChannelStatus deve enviar uma mensagem ChannelStatusAck ao nó downstream indicando o recebimento da mensagem ChannelStatus. O nó upstream deve então, correlacionar a falha para verificar se a falha foi também detectada localmente para o(s) LSP(s) correspondente(s). Se, por exemplo, a falha não existe na entrada do nó upstream ou internamente, então o nó upstream localizou a falha. Assim que a falha é localizada, o nó upstream deve enviar uma mensagem ChannelStatus ao nó downstream indicando se o canal está com uma falha ou se está ok. Se a mensagem ChannelStatus não for recebida pelo nó downstream, ele deve enviar uma mensagem ChannelStatusRequest para o canal em questão. Logo que a falha for localizada, os protocolos de sinalização podem ser usados para iniciar procedimentos de proteção e restauração [PAPADIMITRIOU, 2002], [VASSEUR, 2001].

\subsection{PROCEDIMENTOS DE BACK-OFF EXPONENCIAL}

Esta seção é baseada na RFC2961 e fornece um procedimento de back-off exponencial para retransmissão de mensagens.

A seguinte operação é um dos possíveis mecanismos para retransmissão back-off exponencial de mensagens LMP não reconhecidas. O nó transmissor retransmite a mensagem até que uma mensagem reconhecida seja recebida ou até que um limite de tentativas seja alcançado. Quando o nó transmissor recebe o reconhecimento, a retransmissão da mensagem é cessada. O intervalo entre as retransmissões de mensagens é ditado pelo temporizador de 
retransmissão. O temporizador de retransmissão inicia com um intervalo pequeno e aumenta exponencialmente este intervalo até atingir um limite.

Os seguintes parâmetros de tempo são úteis para caracterizar os procedimentos:

- Intervalo de retransmissão $\left(\mathrm{R}_{\mathrm{i}}\right)$

$\mathrm{R}_{\mathrm{i}}$ é o intervalo de retransmissão inicial para mensagens não reconhecidas. Depois de enviar a mensagem pela primeira vez, o nó transmissor programa uma retransmissão depois de $\mathrm{R}_{\mathrm{i}}$ milisegundos.

- Limite de tentativas $\left(\mathrm{R}_{1}\right)$

$\mathrm{R}_{1}$ é o número máximo de vezes que uma mensagem será transmitida sem ser reconhecida.

- Valor de incremento Delta

Delta determina a velocidade com a qual o transmissor aumenta o intervalo de retransmissão. A proporção de dois intervalos de retransmissão sucessivos é $(1+$ Delta).

Valor default sugerido para o intervalo de retransmissão inicial (Ri) é de 500ms, de Delta $=1$ (exponencial de dois, $1+$ Delta) e um limite de tentativas de valor 3 .

\footnotetext{
Algoritmo de Retransmissão

Depois que um nó transmite uma mensagem requerendo reconhecimento, ele deve imediatamente programar a retransmissão depois de $\mathrm{R}_{\mathrm{i}}$ milisegundos. Se a mensagem de reconhecimento correspondente é recebida antes dos $\mathrm{R}_{\mathrm{i}}$ milisegundos, então a retransmissão da mensagem deve ser cancelada. Caso contrário, ele retransmite a mensagem depois de $(1+$
} 
Delta) $* \mathrm{R}_{\mathrm{i}}$ milisegundos. A retransmissão continua até que a mensagem de reconhecimento apropriada seja recebida ou até que o limite de retransmissão, $\mathrm{R}_{1}$, seja atingido.

Um nó transmissor pode usar o seguinte algoritmo quando for transmitir uma mensagem que requer reconhecimento:

Antes da transmissão inicial, inicializar $\mathrm{R}_{\mathrm{k}}=\mathrm{R}_{\mathrm{i}}$ e $\mathrm{R}_{\mathrm{n}}=0$

Enquanto $\left(\mathrm{R}_{\mathrm{n}}++<\mathrm{R}_{\mathrm{l}}\right)\{$

Transmitir a mensagem;

Acordar depois de $\mathrm{R}_{\mathrm{k}}$ milisegundos;

$\mathrm{R}_{\mathrm{k}}=\mathrm{R}_{\mathrm{k}} *(1+$ Delta $)$

\}

I* mensagem de reconhecimento ou nenhuma resposta do receptor e $\mathrm{R}_{1}$ foi atingido*/

Fazer qualquer limpeza necessária;

Sair;

De forma assíncrona, quando um nó transmissor recebe uma mensagem de reconhecimento correspondente, ele muda o contador de tentativas, $R_{n}$ para $R_{l}$.

\subsection{MÁQUINAS DE ESTADO FINITAS}

\subsubsection{MEF de Canal de Controle}

A MEF de canal de controle define os estados e lógicas de operação de um canal de controle LMP [LANG, 2003]. 


\section{Estados de Canal de Controle}

Um canal de controle pode estar em um dos estados descritos abaixo. Cada estado corresponde a uma certa condição do canal de controle e é normalmente associado com um tipo específico de mensagem que é periodicamente transmitida até a terminação.

- Down

Este é o estado inicial de um canal de controle. Neste estado, nenhuma tentativa está sendo realizada para ativar o canal de controle e nenhuma mensagem LMP é transmitida. Aos parâmetros do canal de controle deve-se atribuir os valores iniciais.

- ConfSnd

O canal de controle está no estado de negociação de parâmetros. Neste estado o nó periodicamente envia uma mensagem Config, e espera que o outro lado responda com uma mensagem ConfigAck ou ConfigNack. A MEF não faz a transição ao estado Active até que o lado remoto reconheça positivamente os parâmetros.

- ConfRcv

O canal de controle está no estado de negociação. Neste estado, o nó está esperando por parâmetros de configuração aceitáveis do lado remoto. Assim que tais parâmetros são recebidos e reconhecidos, a MEF pode realizar a transição ao estado Active.

- Active

Neste estado, o nó periodicamente envia uma mensagem Hello e espera receber uma mensagem Hello válida. Assim que uma mensagem Hello válida é recebida, o nó pode fazer a transição ao estado $U p$.

- $U p$

O canal de controle está no estado operacional. O nó recebe mensagens Hello válidas e envia mensagens Hello.

- GoingDown 
Um canal de controle pode atingir este estado devido a ações administrativas. Enquanto um canal de controle está neste estado, o nó marca o bit ControlChannelDown em todas as mensagens que ele envia.

\section{Eventos de Canal de Controle}

Operações de um canal de controle são descritas em termos de estados e eventos de uma MEF. Eventos de canal de controle são gerados pelos protocolos e módulos de software, como também pelas rotinas de processamento de pacotes e MEFs de enlaces TE associados. Cada evento tem seu número e um nome simbólico. A descrição de possíveis eventos de canal de controle é dada a seguir:

- EvBringUp (1)

Este evento é acionado externamente indicando que a negociação do canal de controle deve ser iniciada. Este pode ser acionado por um comando de operador, ou pelo sucesso no procedimento de bootstrap do canal de controle ou pela configuração. Dependendo da configuração este evento acionará:

a. O envio de uma mensagem Config;

b. Um período de espera para receber uma mensagem Config do nó remoto.

- $\operatorname{Ev} C C D n(2)$

Este evento é gerado quando existe indicação que o canal de controle não está mais disponível

- EvConfDone (3)

Este evento indica que uma mensagem ConfigAck foi recebida reconhecendo os parâmetros de Config. 
- EvConfErr (4)

Este evento indica que uma mensagem ConfigNack foi recebida rejeitando os parâmetros de Config.

- $\quad \operatorname{EvNewConfOK(5)}$

Uma nova mensagem Config foi recebida de um vizinho e positivamente reconhecida.

- $\quad$ EvNewConfErr (6)

Uma nova mensagem Config foi recebida de um vizinho e rejeitada com uma mensagem ConfigNack.

- EvContenWin (7)

Uma nova mensagem Config foi recebida de um vizinho e ao mesmo tempo uma mensagem Config foi enviada ao vizinho. O nó local vence a contenção. Como resultado, a mensagem Config recebida é ignorada.

- EvContenLost (8)

Uma nova mensagem Config foi recebida de um vizinho e ao mesmo tempo uma mensagem Config foi enviada ao vizinho. O nó local perde a contenção.

(a) A mensagem Config é positivamente reconhecida;

(b) A mensagem Config é negativamente reconhecida.

- EvAdminDown (9)

O administrador requer que o canal de controle seja, administrativamente, colocado no estado Down.

- EvNbrGoesDn (10)

Um pacote com a flag ControlChannelDown é recebida de um vizinho.

- EvHelloRcvd (11)

Um pacote Hello com o SeqSum esperado é recebido 
- EvHoldTimer (12)

O temporizador HelloDeadInterval expirou indicando que nenhum pacote Hello foi recebido. Isso faz com que o canal de controle volte ao estado de negociação, e dependendo da configuração local este procedimento iniciará:

(a) O envio de mensagens Config periodicamente;

(b) Um período de espera para receber mensagens Config do nó remoto.

- EvSeqNumErr (13)

Uma mensagem Hello com o SeqNum não esperado é recebida e descartada.

- EvReconfig (14)

Os parâmetros do canal de controle foram reconfigurados e requerem uma nova negociação.

- EvConfRet (15)

O temporizador de retransmissão expirou e uma mensagem Config é retransmitida.

- EvHelloRet (16)

O temporizador HelloInterval expirou e um pacote Hello é enviado.

- EvDownTimer (17)

O temporizador expirou e nenhuma mensagem foi recebida com a flag ControlChannelDown marcada.

\section{Descrição da MEF de Canal de Controle}

A Figura 5.1 ilustra as operações da MEF de canal de controle na forma de um diagrama de transição de estados.

O evento EvCCDn sempre força a MEF ao estado Down. Os eventos EvHoldTimer e EvReconfig sempre forçam a MEF ao estado de negociação (ConfSnd ou ConfRcv). 


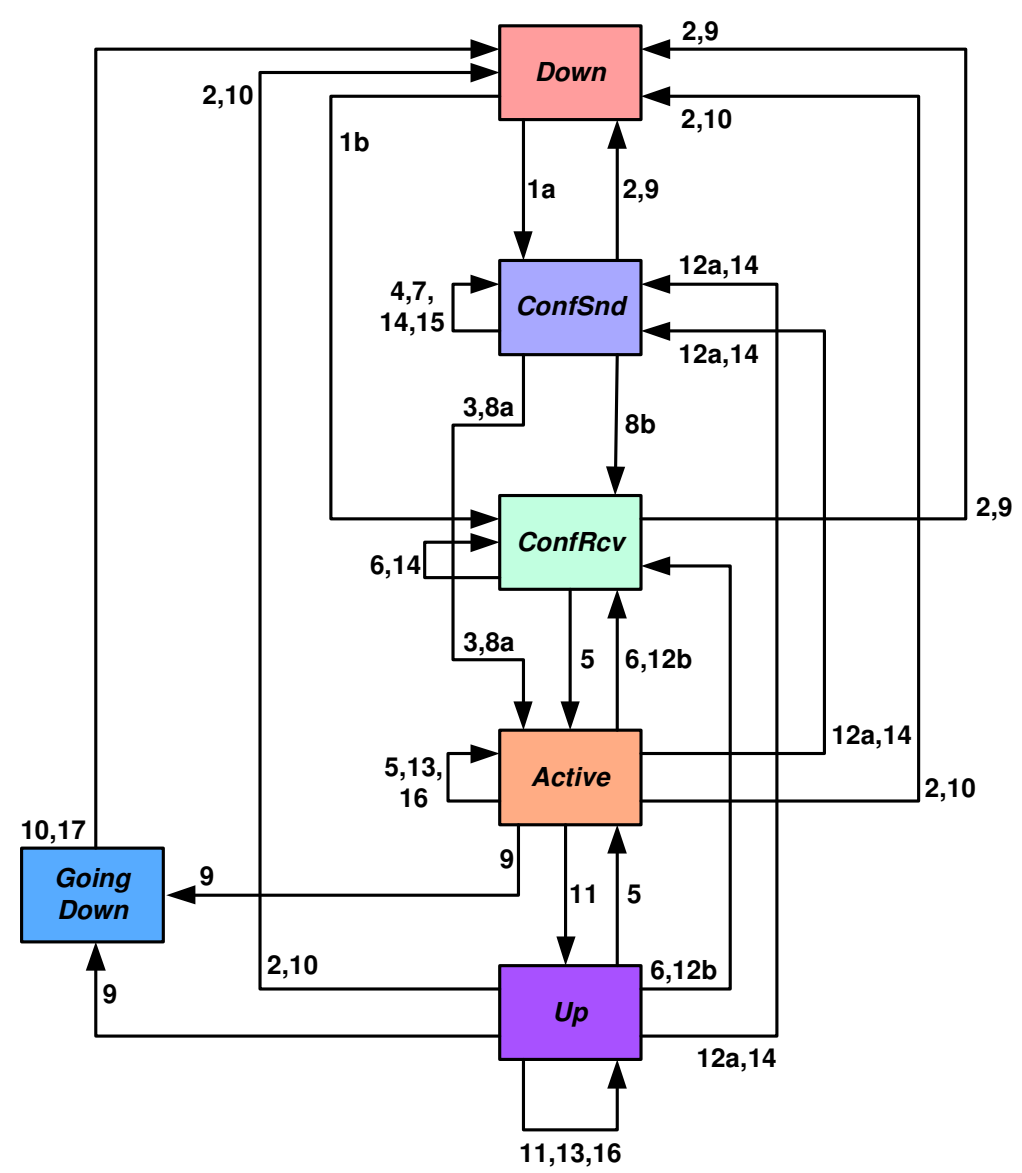

Figura 5.1 - MEF de Canal de Controle

\section{Análise Lógica}

A máquina de estado finita de canal de controle apresentada na Figura 5.1 é baseada em eventos. A partir desta, duas máquinas de estado finitas de comunicação, $M$ e $N$, podem ser geradas e utilizadas para análise lógica do LMP relacionado ao canal de controle.

A listagem dos eventos utilizados durante a comunicação na rede de $\operatorname{MEFCs}[M, N]$ é apresentada na parte inferior da Figura 5.2. As mensagens citadas já foram mencionadas e definidas anteriormente e representam agora apenas uma ação ao invés de duas ou mais ações como na Figura 5.1. 

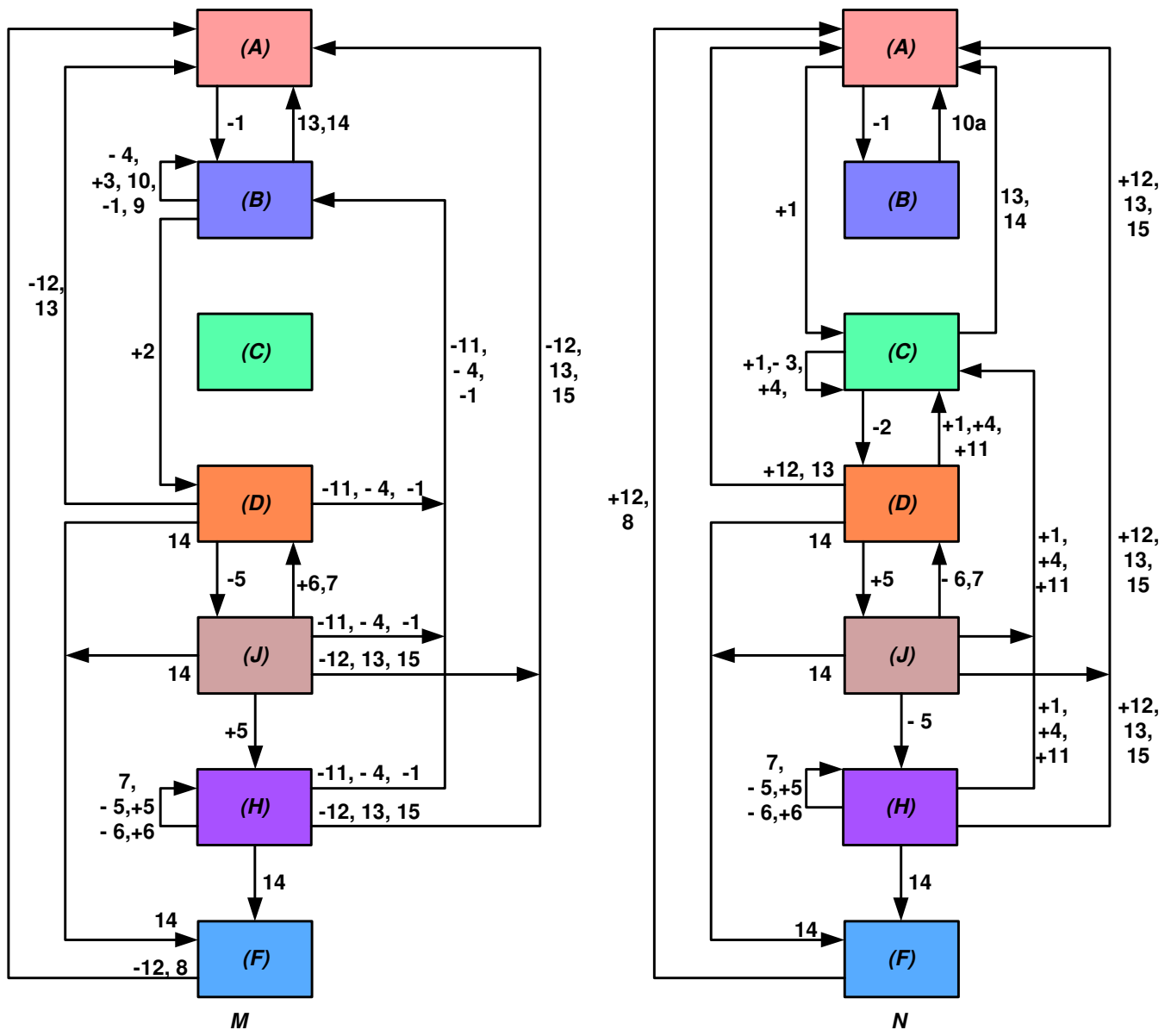
(1) Config
(3) ConfigNack
(5) Hello
(7) Temporizador Hello expirou
(9) Temporizador de retransmissão expirou
(10a) Nó local perde contenção

(12) Pacote com flag ControlChannelDown é recebido

(14) Canal de controle colocado em down administrativamente

(15) HelloDeadInterval expirou

(2) ConfigAck

(4) NewConfig

(6) Hello (com número de sequência errado)

(8) Temporizador expirou

(10) Nó local vence contenção

(11) Negociação

(13) Canal de controle não está disponível

Figura 5.2 - Rede de MEFCs de canal de controle formada a partir da MEF de canal de controle $^{14}$

Com base na rede de MEFCs apresentada na Figura 5.2, é possível aplicar a técnica de análise lógica denominada análise com progresso justo e verificar se a parte do protocolo LMP relacionado com o canal de controle possui ou não erros de progresso.

\footnotetext{
${ }^{14} \mathrm{O}$ estado E é propositalmente evitado pois E significa canal de comunicação vazio como mencionado no capítulo IV
} 
O resultado desta análise é a construção do grafo de alcançabilidade justa, como mostra a Figura 5.3. As mensagens e seus números correspondentes utilizados no grafo são os mesmos utilizados na rede de MEFCs, apresentada na Figura 5.2. De acordo com a grafo e com base nas definições de erros de progresso apresentadas anteriormente, nota-se que o LMP relacionado ao canal de controle não apresenta deadlocks, recepções não especificadas nem mesmo, interações não-executadas, livelocks e overflow.

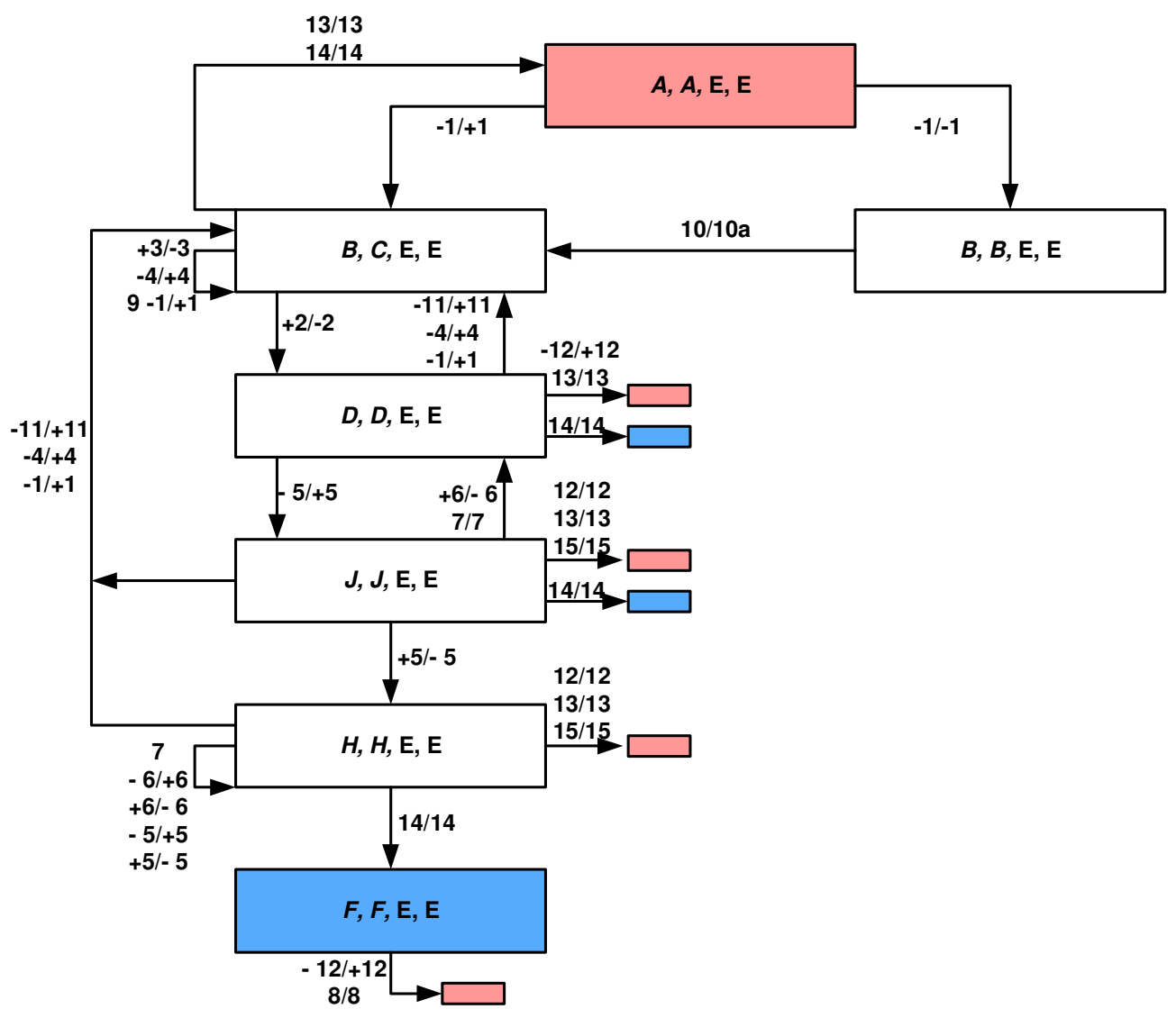

Figura 5.3 - Grafo de Alcançabilidade Justa resultante da rede de MEFCs do LMP relacionado ao canal de controle.

\subsubsection{MEF de Enlace TE}

A MEF de enlace TE define os estados e lógicas de operação do enlace TE. 


\section{Estados de Enlace TE}

Um enlace TE pode estar em um dos estados descritos a seguir. Cada estado corresponde a uma certa condição do enlace TE e é normalmente associado com um tipo específico de mensagem LMP que é periodicamente transmitida à terminação associada ao canal de controle.

- Down

Não existem enlaces de dados alocados ao enlace TE.

- Init

Enlaces de dados foram alocados ao enlace TE, mas a configuração ainda não foi sincronizada com o vizinho LMP. A mensagem LinkSummary é periodicamente transmitida ao vizinho LMP.

- $U p$

Este é o estado de operação normal do enlace TE. Pelo menos um canal de controle LMP deve estar operando entre os nós que compartilham o enlace TE. Como parte da operação normal, a mensagem LinkSummary pode ser periodicamente transmitida ao vizinho LMP ou gerada por um pedido externo.

- Degraded

Neste estado, todos os canais de controle LMP estão Down, mas o enlace TE ainda inclui alguns enlaces de dados que estão alocados para tráfego de usuário.

\section{Eventos de Enlace TE}

Operações de um enlace TE são descritas em termos de estados de MEF e eventos. Eventos de enlace TE são gerados por rotinas de processamento de pacotes e por MEFs do(s) canal(is) de controle e enlaces de dados associados. Cada evento tem seu número e um nome simbólico. A descrição de possíveis eventos é dada a seguir: 
- $\operatorname{EvDCUp}(1)$

Um ou mais canais de dados foram liberados e atribuídos a um enlace TE.

- EvSumAck (2)

Mensagem LinkSummary foi recebida e positivamente reconhecida.

- EvSumNack (3)

A mensagem LinkSummary é recebida e negativamente reconhecida.

- $\operatorname{EvRcvAck}(4)$

A mensagem LinkSummaryAck é recebida reconhecendo a configuração do enlace TE.

- $\operatorname{EvRcvNack(5)}$

A mensagem LinkSummaryNack foi recebida.

- EvSumRet (6)

O temporizador de retransmissão expirou e a mensagem LinkSummary é reenviada.

- $\operatorname{Ev} C C U p(7)$

Primeiro canal de controle ativo fica $u p$.

- $\quad$ EvCCDown (8)

Último canal de controle ativo fica down.

- $\operatorname{EvDCDown}(9)$

Último canal de dados do enlace TE foi removido.

\section{Descrição da MEF de Enlace TE}

A Figura 5.4 ilustra as operações de um MEF de enlace TE na forma de um diagrama de transição de estados.

$\mathrm{Na} \mathrm{MEF}$ recém mencionada, os sub-estados que poderiam ser implementados quando o procedimento de verificação de enlace é usado foram omitidos. 


\section{Análise Lógica}

A máquina de estado finita de enlace TE apresentada na Figura 5.4 é baseada em eventos. A partir desta, duas máquinas de estado finitas de comunicação, $M$ e $N$, podem ser geradas e utilizadas para análise lógica do LMP relacionado a enlace TE. Na rede $[M, N]$ os eventos definidos representam apenas uma ação, ao contrário dos eventos da Figura 5.4 que representam duas ou mais ações.

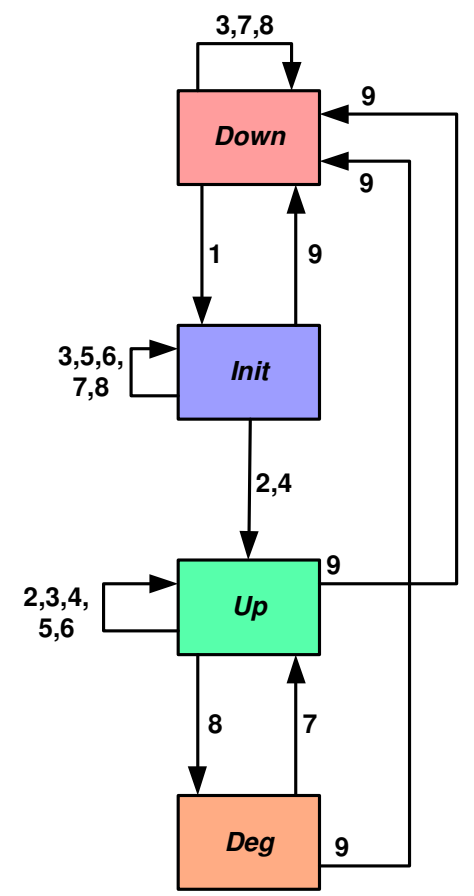

Figura 5.4 - MEF de enlace TE

A listagem dos eventos utilizados na comunicação na rede de MEFCs $[M, N]$ são apresentados na base da Figura 5.5. As mensagens citadas já foram mencionadas e definidas anteriormente.

Com base na rede de MEFCs apresentada na Figura 5.5, é possível aplicar a análise com progresso justo e verificar se o LMP relacionado com ao enlace TE possui ou não erros de progresso. 


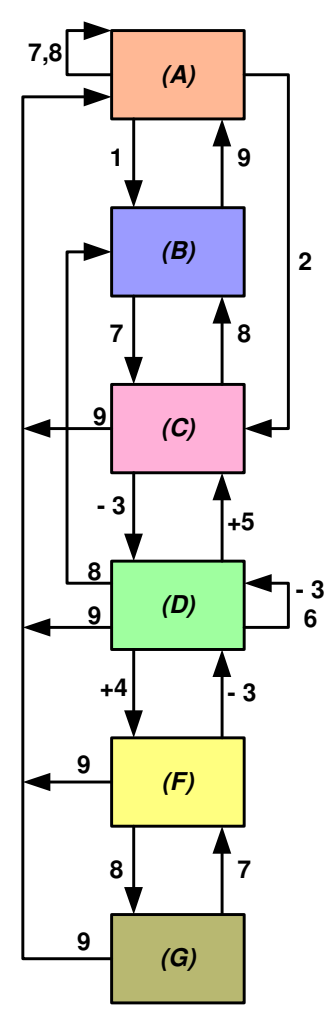

$M$

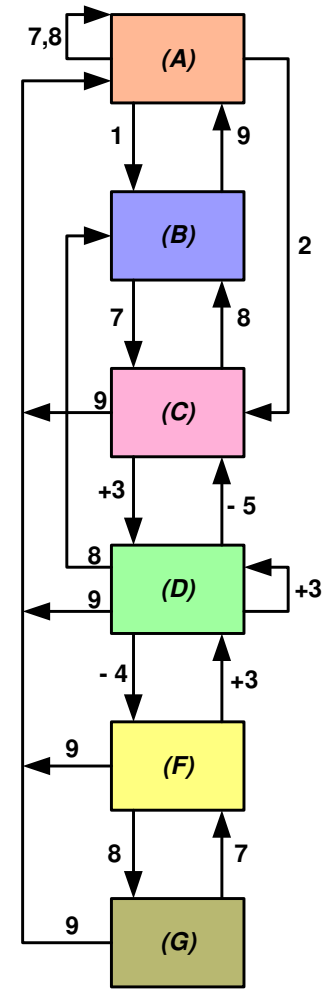

N

(1) Enlace de dados atribuídos ao enlace TE, mas não há canal de controle up

(2) Enlace de dados atribuídos ao enlace TE e existe canal de controle up

(3) LinkSummary

(4) LinkSummaryAck

(5) LinkSummaryNack

(6) Temprizador expirou

(7) Primeiro canal de controle fica up

(8) Último canal de controle fica down

(9) Último enlace de dados é removido

Figura 5.5 - Rede de MEFCs de enlace TE formada a partir da MEF de enlace TE

O resultado desta análise é a construção do grafo de alcançabilidade justa, como mostra a Figura 5.6. As mensagens e suas correspondentes numerações utilizadas no grafo de alcançabilidades são as mesmas utilizadas na Figura 5.5. De acordo com a grafo e com base nas definições de erros de progresso apresentadas anteriormente, nota-se que o LMP relacionado ao enlace TE não apresenta nenhum dos cinco erros de progresso. 


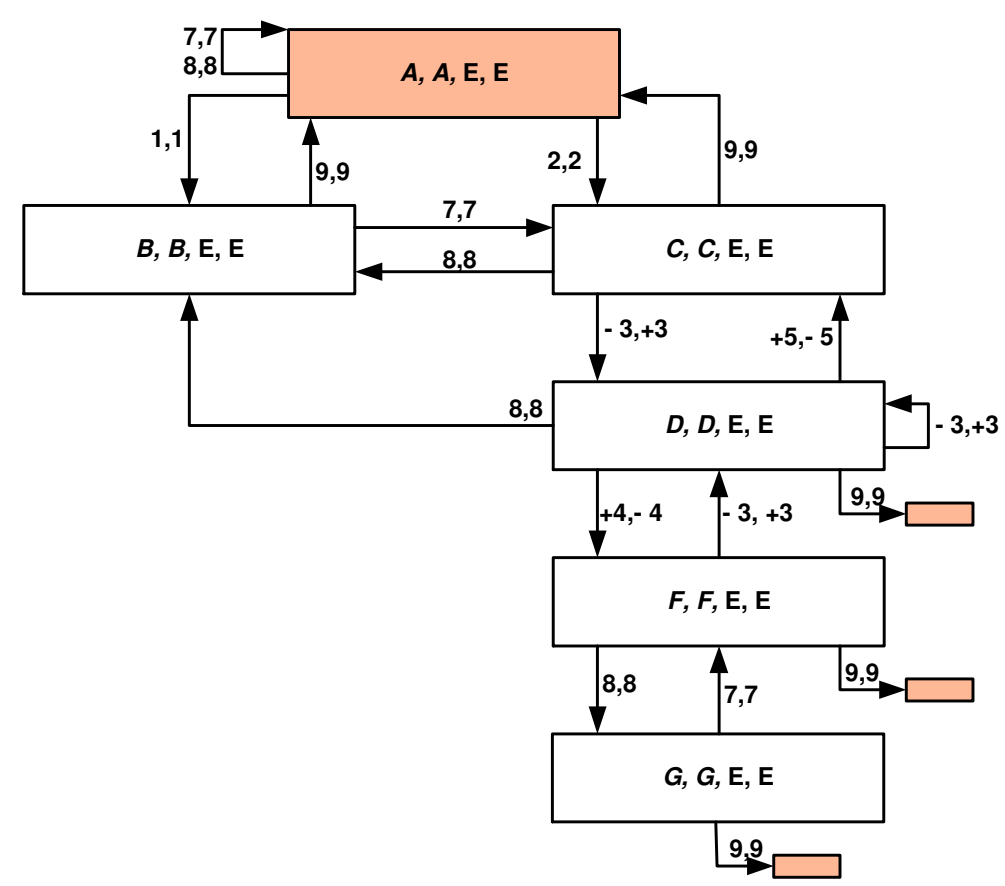

Figura 5.6 - Grafo de Alcançabilidade Justa resultante da rede de MEFCs do LMP relacionado ao enlace TE.

\subsubsection{MEF de Enlace de Dados}

A MEF de enlace de dados define estados e lógicas de operação de um enlace de dados no enlace TE. Operações de um enlace de dados são descritas em termos de estados e eventos de MEF. Enlaces de dados podem estar no modo ativo, onde mensagens Test são transmitidas, ou no modo passivo, onde mensagens Test são recebidas. Para facilitar a compreensão, MEFs separadas são definidas para enlaces de dados ativos/passivos, no entanto, um único conjunto de estados e eventos de enlaces de dados são definidos.

\section{Estados de Enlace de Dados}

Qualquer enlace de dados pode estar em qualquer um dos estados descritos a seguir. Cada estado corresponde a uma certa condição do enlace de dados. 
- Down

O enlace de dados não foi colocado no reservatório de recursos (i.e., o enlace não está em serviço).

- Test

O enlace de dados está sendo testado. Uma mensagem Test é periodicamente enviada através do enlace.

- PasvTest

O enlace de dados está sendo verificado para mensagens Test de entrada.

- Up/Free

O enlace foi testado com sucesso e é agora colocado no reservatório de reservas (em serviço). O enlace ainda não foi alocado para tráfego de dados.

- Up/Alloc

O enlace está up e foi alocado para tráfego de dados.

\section{Eventos de Enlace de Dados}

Eventos de enlaces de dados são gerados por rotinas de processamento de pacotes e pelas MEFs dos enlaces TE e canais de controle associados.

Cada evento tem seu número e um nome simbólico. Descrição de possíveis eventos de enlaces de dados é fornecida a seguir:

- $\operatorname{EvCCUp}(1)$

Primeiro canal de controle está up.

- EvCCDown (2)

A conectividade com o vizinho LMP foi perdida. Isto indica que o último canal de controle entre os nós vizinhos falhou. 
- $\quad$ EvStartTst (3)

Este é um evento externo que aciona o envio de mensagens Test sobre o enlace de dados.

- EvStartPsv (4)

Este é um evento externo que aciona a escuta por mensagens Test no enlace de dados.

- $\operatorname{EvTestOK}(5)$

Verificação de enlace obteve sucesso e o enlace pode ser usado para estabelecimento de caminho.

(a) Este evento indica que o procedimento de verificação de enlace obteve sucesso para este enlace de dados e uma mensagem TestStatusSuccess foi recebida sobre o canal de controle.

(b) Este evento indica que o enlace está pronto para o estabelecimento de caminho, mas o procedimento de verificação de enlace não foi usado. Para sinalização inband do canal de controle, o estabelecimento do canal de controle pode ser suficiente para verificar o enlace.

- $\operatorname{EvTestRcv(6)}$

A mensagem Test foi recebida na porta de dados e uma mensagem TestStatusSuccess foi transmitida sobre o canal de controle.

- EvTestFail (7)

Verificação de enlace retornou resultados negativos. Isto pode ser devido (a) ao recebimento de uma mensagem TestStatusFailure, ou (b) a finalização do processo de verificação sem o recebimento de uma mensagem TestStatusSuccess ou TestStatusFailure para o enlace de dados. 
- EvPsvTestFail (8)

A verificação de enlace retornou resultados negativos. Isto indica que uma mensagem Test não foi detectada e, ou (a) o VerifyDeadInterval expirou, ou (b) o procedimento de verificação finalizou e o VerifyDeadInterval ainda não expirou.

- EvLnkAlloc (9)

O enlace de dados foi alocado.

- EvLnkDeadloc (10)

O enlace de dados foi desalocado.

- EvTestRet (11)

O temporizador de retransmissão expirou e a mensagem Test foi re-enviada.

- EvSummaryFail (12)

Não foi verificada igualdade entre a mensagem LinkSummary e a porta de dados.

- EvLocalizeFail (13)

Uma falha foi localizada no enlace de dados.

- EcDCDown (14)

O canal de dados não está disponível.

\section{Descrição da MEF de Enlace de Dados Ativo}

A Figura 5.7 ilustra a operação de uma MEF de enlace de dados ativo na forma de um diagrama de transição de estado.

\section{Descrição da MEF de Enlace de Dados Passivo}

A Figura 5.8 ilustra a operação de um MEF de enlace de dados passivo na forma de um diagrama de transição de estados. 
Com base nas definições dos eventos utilizados nas MEFs de enlace de dados (ativo e passivo) e nas Figuras 5.7 e 5.8 é fácil notar que os eventos (1) e (2) foram definidos mas não usados. Conseqüentemente, antes mesmo da análise lógica ser aplicada, nota-se a presença do erro de progresso denominado interação não-executada.

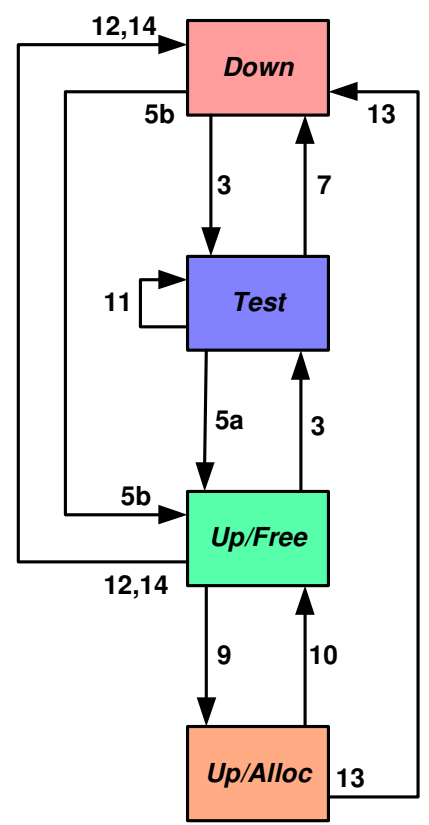

Figura 5.7 - MEF de Enlace de Dados Ativo

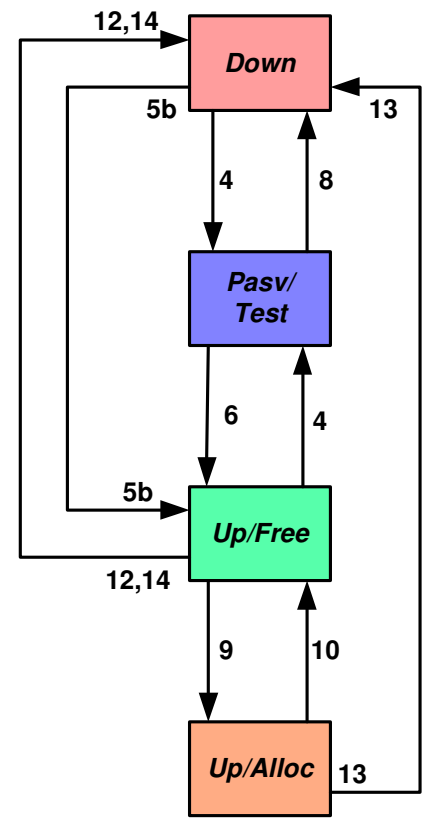

Figura 5.8 - MEF de Enlace de Dados Passivo 
O Internet Draft [ccamp-lmp-10] que apresenta estes MEFs falha por não apresentar, como parte das MEFs, o início do procedimento de verificação de enlace que inclui a troca de mensagens BeginVerify, BeginVerifyAck e BeginVerifyNack. Esta parte do procedimento foi corretamente incluída nesta tese quando a rede de MEFCs foi construída para que a análise lógica deste procedimento pudesse ser desempenhada.

\section{Análise Lógica}

As máquinas de estado finita de enlaces de dados, ativo e passivo, apresentadas nas Figura 5.7 e 5.8 respectivamente, são baseadas em eventos que representam, em sua maioria, duas ou mais ações. A partir destas MEFs, duas máquinas de estado finitas de comunicação, $M$ e $N$, podem ser geradas e utilizadas para análise lógica do LMP relacionado ao enlace de dados passivo e enlace de dados ativo. A rede de MEFCs, que une os eventos dos enlaces de dados ativos e passivos, é construída para que os eventos possam representar apenas uma ação e tornar possível a aplicação da técnica de análise justa.

A Figura 5.9, na parte inferior da mesma, apresenta a listagem dos eventos utilizados na comunicação na rede de MEFCs $[M, N]$. As mensagens citadas já foram mencionadas e definidas anteriormente.

Com base na rede de MEFCs apresentada na Figura 5.9, é possível aplicar a técnica de análise com progresso justo e verificar se o LMP relacionado com enlace de dados (ativo e passivo) possui ou não um dos cinco erros de progresso. 


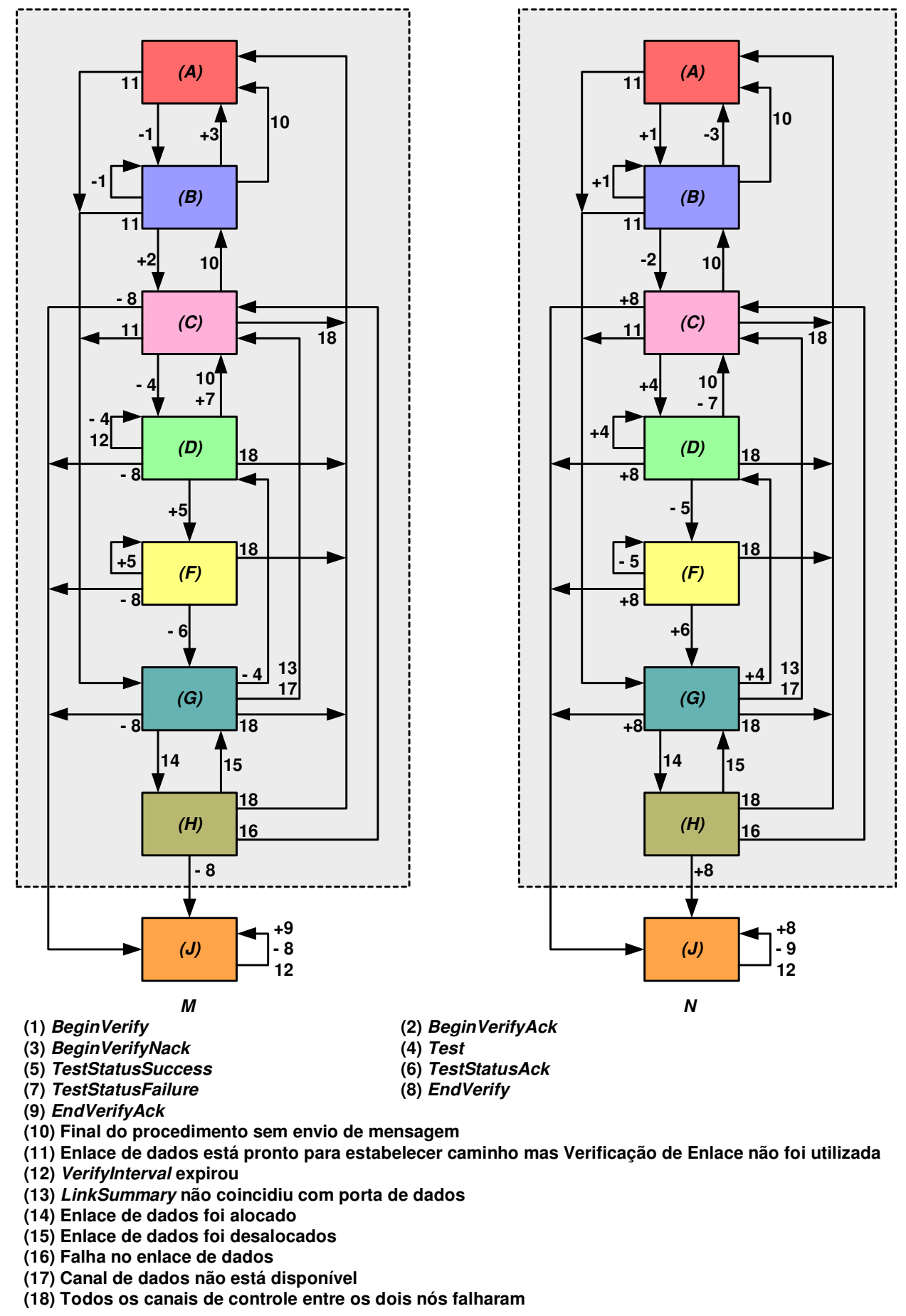

Figura 5.9 - Rede de MEFCs de enlace de dados (ativo e passivo) formada a partir das MEFs de enlaces de dados ativo e passivo

O grafo de alcançabilidade justa, como mostra a Figura 5.10, é o resultado da análise justa. As mensagens utilizadas no grafo de alcançabilidade são as mesmas mensagens apresentadas na rede de MEFCs para o LMP relacionado ao enlace de dados. De acordo com a grafo e com 
base nas definições de erros de progresso apresentadas anteriormente, nota-se que o LMP relacionado ao enlace de dados (ativo e passivo) não apresenta nenhum dos cinco erros de progresso.

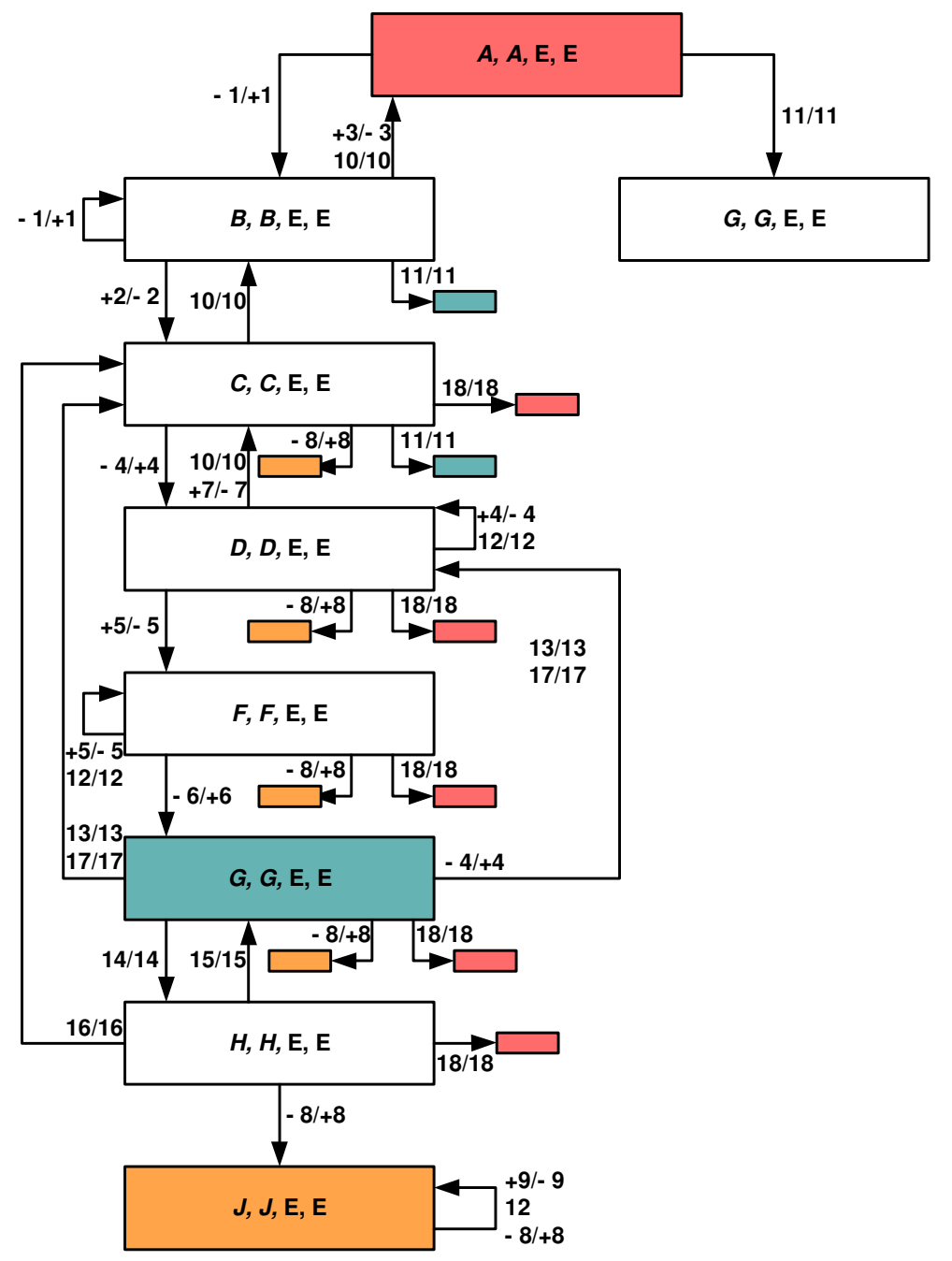

Figura 5.10 - Grafo de Alcançabilidade Justa resultante da rede de MEFCs do LMP relacionado ao enlace de dados passivo e ativo

\subsubsection{MEF de Gerenciamento de Falhas}

A MEF de falhas define estados e lógicas de operação do procedimento de gerenciamento de falhas. Operações de gerenciamento de falhas são descritas em termos de estados e eventos de MEF. 


\section{Análise Lógica}

As máquinas de estado finita de gerenciamento de enlace apresentadas na Figura 5.11 formam a rede de MEFCs que é baseada em eventos que representam apenas uma ação, o que torna possível a aplicação da técnica de análise justa.

As mensagens e eventos utilizados na rede de MEFCs são mencionadas na própria Figura 5.11 e os mesmos já foram mencionados e definidos anteriormente.

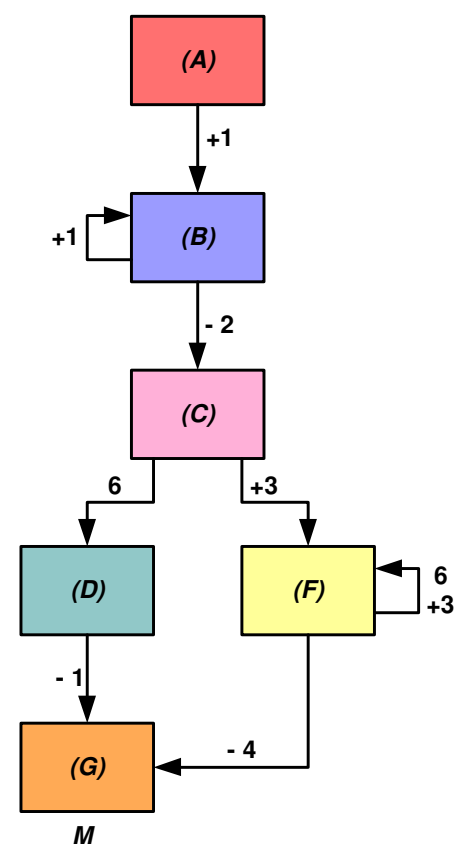

$$
\begin{aligned}
& \text { (1) ChannelStatus } \\
& \text { (3) ChannelStatusRequest } \\
& \text { (5) Temporizador expirou } \\
& \text { (7) Espera de resposta }
\end{aligned}
$$

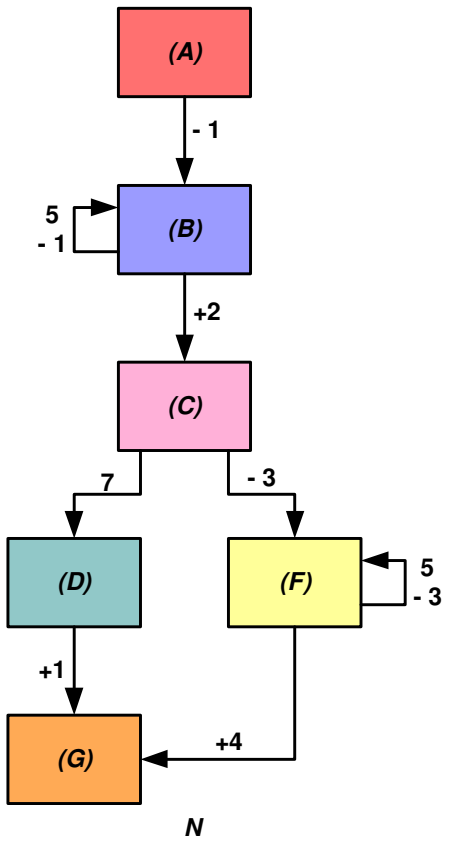

(2) ChannelStatusAck
(4) ChannelStatusResponse
(6) Correlação de falhas

Figura 5.11 - Rede de MEFCs de gerenciamento de falhas

Com base na rede de MEFCs apresentada na Figura 5.11, é possível aplicar a técnica de análise com progresso justo e verificar se o LMP relacionado com o gerenciamento de falhas possui ou não erros de progresso. 
O resultado desta análise é a construção do grafo de alcançabilidade justa, como mostra a Figura 5.12. As mensagens utilizadas no grafo de alcançabilidade são as mesmas mensagens apresentadas na rede de MEFCs para o LMP relacionado ao gerenciamento de falhas. De acordo com a grafo e com base nas definições de erros de progresso apresentadas anteriormente, nota-se que o LMP relacionado ao gerenciamento de falhas não apresenta nenhum dos cinco erros de progresso.

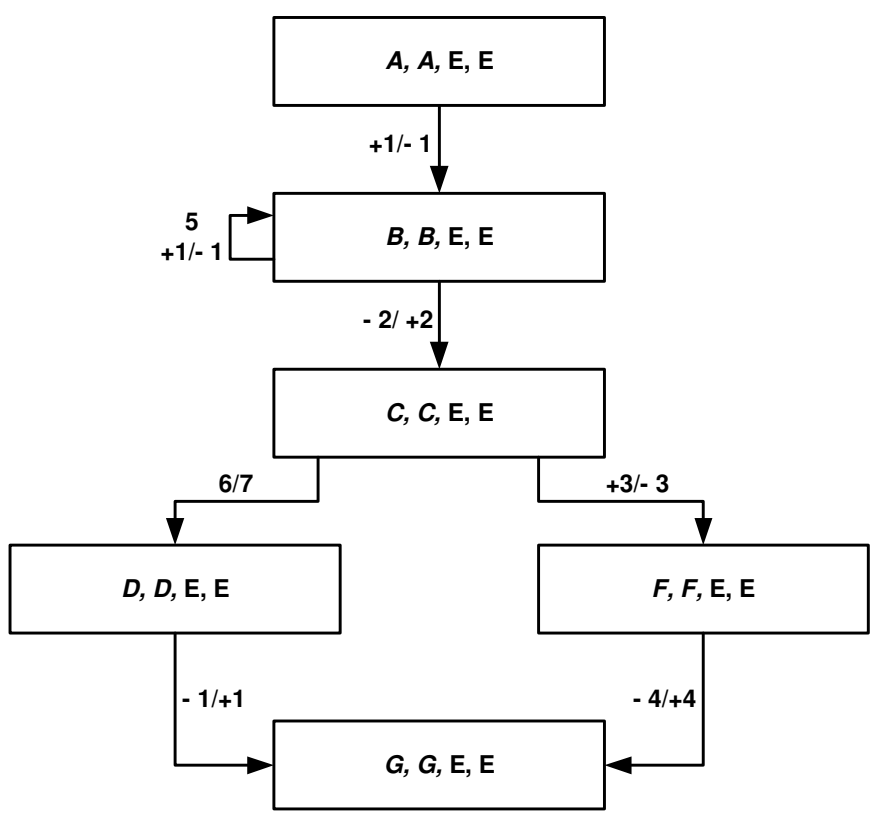

Figura 5.12 - Grafo de Alcançabilidade Justa resultante da rede de MEFCs do LMP relacionado ao gerenciamento de falhas

\subsection{CONCLUSÃO}

O protocolo de gerenciamento de enlace (LMP - Link Management Protocol) é executado entre um par de nós vizinhos e é usado para gerenciar enlaces TE e verificar a alcançabilidade do canal de controle. De forma mais detalhada, suas funções são: manter a conectividade do canal de controle, verificar a conectividade física dos enlaces de dados, correlacionar as 
informações de propriedades de enlace, suprimir alarmes downstream desnecessários e, localizar falhas de enlace para finalidade de proteção/restauração em múltiplos tipos de redes.

Com base nas definições e especificações de cada procedimento LMP, propõe-se uma ordem de execução destes procedimentos:

(i) Gerenciamento de canal de controle

- Ativação do canal de controle

- Manutenção do canal de controle

(ii) Verificação de conectividade de enlace

- Pelo menos um canal de controle deve estar ativo

- Descobrimento dinâmico das associações entre Interface_IDs local e remoto de um mesmo enlace de dados

- Verificação de conectividade de enlaces de dados

- Descobrimento dinâmico de associações de enlace TE

(iii) Correlação de Propriedade de enlace

- Mapeamento de Interface_IDs deve ser um conhecimento já adquirido pelo nó local

- Agregação de múltiplos enlaces de dados a um enlace TE

- Sincronização de propriedades de enlace

(iv) Gerenciamento de Falhas

- Notificação do status de enlaces

- Localização de falhas

Esta ordem deve ser seguida quando a rede for colocada em funcionamento pois nesse caso, a maior parte das informações serão geradas e descobertas dinamicamente. No decorrer do 
funcionamento da rede, essa ordem de execução dos procedimentos LMP não necessariamente precisa ser seguida na maioria dos possíveis cenários.

O funcionamento do LMP é especificado através de máquinas de estado finitas de comunicação para cada um dos procedimentos do protocolo de gerenciamento de enlace. As MEFC são utilizadas para verificar e analisar logicamente o protocolo com relação aos erros de progresso (deadlock, recepção não-especificada, interação não-executada, livelock e overflow). Para esta finalidade, a técnica de análise justa é eficientemente utilizada.

Com base nos grafos de alcançabilidade justa gerados pela análise com progresso justo, o protocolo de gerenciamento de enlace utilizado em um ambiente GMPLS está livre dos erros de progresso.

Note que para tornar o LMP livre de erros de progresso e fazer com que este tenha um melhor sucesso na ordem de execução e lógica de execução, alguns pequenos detalhes de especificação foram corrigidos e outros sugeridos. Essas possíveis correções e sugestões foram apresentadas no decorrer do capítulo. 


\section{CAPÍTULO VI}

\section{ATRIBUIÇÃO DE RÓTULOS COM BASE NA INFORMAÇÃO SRLG EM UM AMBIENTE GMPLS-WDM}

\subsection{INTRODUÇÃO}

Com o uso de conversores de comprimento de onda, em um ambiente GMPLS baseado em WDM, tem-se a vantagem de uma melhor utilização dos comprimentos de onda e conseqüentemente há minimização da probabilidade de bloqueio de conexões, pois se aperfeiçoa a eficiência da rede ao resolver os conflitos de comprimentos de onda durante o estabelecimento de conexões. Porém, a tecnologia de conversores de comprimento de onda ainda não está amadurecida e competitiva e os mesmos possuem um custo muito alto, como mencionado anteriormente. Por esta razão, o foco deste estudo é redes GMPLS baseadas em WDM sem capacidade de conversão de comprimento de onda.

Como o encaminhamento de tráfego deve ser feito sem conversão de comprimento de onda, a propriedade de restrição de continuidade de comprimento de onda deve ser considerada. Logo, um caminho óptico tem que usar o mesmo comprimento de onda em todos os enlaces 
ópticos que o compõem, da origem ao destino. Múltiplos caminhos podem usar o mesmo comprimento de onda (o mesmo rótulo, pois o rótulo representa um comprimento de onda) desde que eles não se sobreponham em uma mesma fibra, evitando assim colisões. O desempenho deste tipo de rede, com relação a probabilidade de bloqueio de conexão, é menor se comparado com o desempenho das redes que possuem capacidade de conversão de comprimento de onda, pois mesmo uma requisição sendo válida, ela pode ser bloqueada caso não encontre um comprimento de onda que esteja disponível simultaneamente em todos os enlaces requisitados, mesmo existindo, em todos eles, comprimentos de onda disponíveis.

A escolha de um bom algoritmo de atribuição de rota e comprimento de onda pode reduzir a freqüência dessas ocorrências, ocasionando um melhor desempenho da rede que assim passa a apresentar uma menor taxa de probabilidade de bloqueio de conexão.

Com o uso do SRLG é fatível selecionar uma rota considerando a separação de estrutura lógica e de recursos. O SRLG pode ser usado para determinar quais caminhos, a serem estabelecidos, são disjuntos com relação a uma entidade de risco específica e ainda considera o problema de traps (Capítulo II, seção 2.7).

Na tentativa de melhorar o desempenho das redes GMPLS baseadas em WDM com restrição de continuidade de comprimento de onda, pode-se expandir o conceito SRLG que passa a ser utilizado para o estabelecimento de todos os caminhos, especificamente na atribuição de comprimento de onda, juntamente com o conceito de conjunto de rótulos. 


\subsection{PROPOSTA}

A idéia para alocação de comprimentos de onda é que para conexões cujos caminhos ópticos compartilham pelo menos um enlace na rede, deve-se atribuir diferentes comprimentos de onda para cada conexão, pois a propriedade de restrição de continuidade de comprimento de onda é considerada. Esta é a mesma idéia proposta pelo conceito SRLG para o estabelecimento de caminhos de proteção e que agora será usado para alocação de comprimento de onda, como proposta deste estudo. Com base na informação SRLG do caminho a ser estabelecido e dos caminhos já existentes tem como saber se estes compartilham ou não alguma entidade de risco (recurso) e assim decidir qual comprimento de onda deve ser atribuído.

Conexões que possuem o mesmo conjunto SRLG, ou pelo menos um dos enlaces desses caminhos ópticos dessas conexões possuem o mesmo SRLG-ID, significa que essas conexões compartilham entidades de risco. Caso a entidade de risco considerada seja apenas um segmento de fibra óptica, durante o estabelecimento de um caminho óptico, se o mesmo cruzar com caminhos já existentes, ou seja, a interseção do conjunto SRLG desses caminhos é diferente de vazio, conseqüentemente um comprimento de onda diferente do comprimento de onda dos caminhos cuja interseção do conjunto SRLG é diferente de vazio, deve ser atribuído ao novo caminho sendo estabelecido.

Assim, caminhos que compartilham enlaces (caminhos com pelo menos um SRLG-ID em comum) recebem diferentes comprimentos de onda e atendem a propriedade de restrição de continuidade de comprimento de onda. 
O conceito conjunto de rótulos, quando aplicado sozinho, produz o mesmo resultado do conceito SRLG, em alguns cenários. No entanto, o conceito de conjunto de rótulos usado isoladamente não consegue atender o problema de trap e por outro lado, SRLG mais conjunto de rótulos juntos conseguem produzir resultados corretos de possíveis comprimentos de ondas a serem escolhidos para atribuição, mesmo quando o problema de trap existe. Desta forma, consegue-se diminuir a probabilidade de bloqueio de conexão das redes GMPLS-WDM sem capacidade de conversão de comprimento de onda.

\subsubsection{Algoritmo Proposto}

Segue o algoritmo proposto que utiliza informações SRLG juntamente com o conjunto de rótulos para atribuição de comprimento de onda.

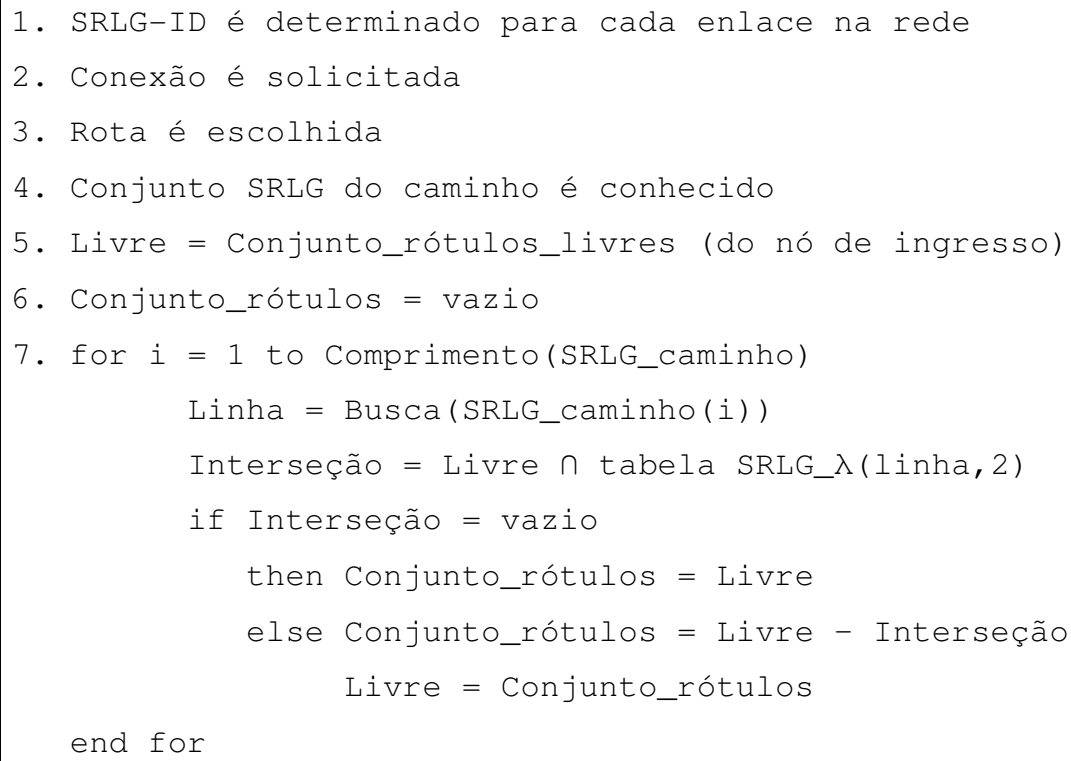




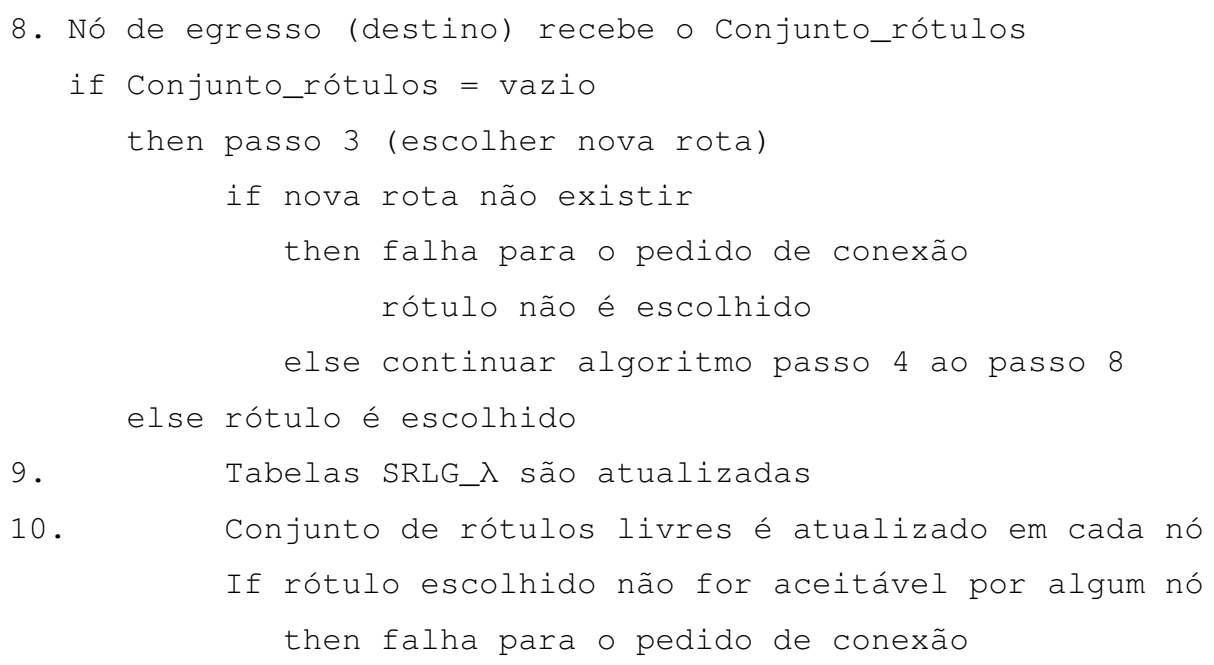

Onde:

- Conjunto_rótulos - conjunto de comprimentos de onda selecionados ao longo do caminho a ser estabelecido para possível escolha durante a atribuição de comprimento de onda

- Conjunto_rótulos_livres - conjunto de comprimentos de onda disponíveis para uso em um determinado nó da rede

- Livre - variável auxiliar

- Linha - contém o número de uma linha da tabela SRLG_ $\lambda$

- Interseção - contém o resultado da interseção entre os comprimentos de onda contidos na variável Livre e os comprimentos de onda utilizados por uma entidade de risco específica representada por um SRLG-ID na tabela SRLG_ $\lambda$

- SRLG_caminho - conjunto de SRLG-IDs de todos os enlaces que compõem um caminho

- SRLG_ $\lambda$ - tabela que contém cada SRLG-ID e os comprimentos de onda utilizados nas entidades de risco representadas pelo SRLG-ID. Esta tabela está presente em cada nó da rede e contém a informação relacionada com um nó específico, ou seja, contém apenas o SRLG-ID e respectivos comprimentos de onda utilizados nas entidades de 
risco que passam por aquele nó e não de todos os nós da rede. Desta forma, a proposta de atribuição de comprimento de onda se aproxima mais do funcionamento do conjunto de rótulos, isoladamente. A Tabela 6.1 apresenta um esboço da tabela SRLG_ $\lambda$.

Tabela 6.1 - Esboço da tabela SRLG_ $\lambda$

\begin{tabular}{|c|c|}
\hline \multicolumn{2}{|c|}{ Tabela SRLG_ $\boldsymbol{\lambda}$} \\
\hline SRLG-ID & $\lambda$ \\
\hline 1 & $\bar{\lambda}$ \\
\hline 2 & $\lambda_{1} \lambda_{2}$ \\
\hline 3 & $\lambda_{1}$ \\
\hline
\end{tabular}

- Comprimento - função que determina o número de elementos de uma variável que representa um conjunto

- Busca - função que retorna a linha na tabela SRLG_ $\lambda$ onde se encontra um SRLG-ID particular

Segue o detalhamento do algoritmo:

Passo 1 - O SRLG-ID é estabelecido para cada enlace (segmento óptico) na rede antes desta ser colocada em funcionamento. Este valor pode ser alterado dinamicamente de acordo com mudanças na rede, caso contrário, permanece inalterado.

Passo 2 - Um pedido de caminho é solicitado, ou seja, uma requisição de conexão chega ao nó de ingresso da rede.

Passo 3 - Caminho de roteamento é determinado usando roteamento fixo alternativo ou roteamento adaptativo. Este caminho é apenas escolhido, ainda não é estabelecido, pois pode não haver rótulo disponível em todo o caminho e por conseqüência a rota não será estabelecida.

Passo 4 - SRLG-IDs dos enlaces do caminho a ser estabelecido são conhecidos e assim o SRLG-caminho é formado.

Passo 5 - Atribui-se todos os rótulos disponíveis do nó de ingresso à variável Livre. 
Passo 6 - O Conjunto_rótulos é inicializado como vazio.

Passo 7 - De acordo com o algoritmo, em cada nó do caminho a ser estabelecido, é necessário fazer a interseção entre o Conjunto_rótulos_livres e possíveis de serem utilizados e os comprimentos de onda em uso naquele enlace particular, representado por um SRLG-ID. Esta informação é mantida na tabela SRLG_ $\lambda$ e é obtida através do SRLG-ID do enlace em questão. Depois de feita a interseção, um novo conjunto de rótulos é construído retirando os comprimentos de onda que não mais podem ser utilizados para o caminho sendo estabelecido. Este procedimento é realizado em cada nó da rota (cada repetição do comando for) ao longo do ambiente GMPLS-WDM.

Passo 8 - Quando o procedimento é finalizado (nó de egresso recebe o Conjunto_rótulos), o nó de destino escolhe um dos rótulos (comprimentos de onda) já previamente selecionados no Conjunto_rótulos. Utiliza-se uma das heurísticas de atribuição de rótulos, First-fit, apresentada anteriormente. Se o Conjunto_rótulos estiver vazio, tenta-se encontrar outra rota. Se não existe outra rota, então a falha ocorre.

Passo 9 e 10 - Com base no rótulo escolhido, as tabelas SRLG_ $\lambda$ de cada nó pertencente ao caminho são atualizadas através do envio da mensagem Resv que simultaneamente faz a atribuição do comprimento onda para aquele caminho específico. Ao mesmo tempo, o conjunto de rótulos livres (disponíveis) e o conjunto de rótulos em uso mantido por cada nó da rede são atualizados nos nós que compõem o caminho estabelecido.

\subsubsection{Análise Lógica}

É necessário verificar a corretude lógica do protocolo de sinalização e LMP em um ambiente GMPLS com o uso do conceito SRLG para auxílio na atribuição de comprimento de onda na tentativa de diminuir a probabilidade de bloqueio de conexão na atribuição de rótulos. 
O SRLG, depois de determinado para cada enlace na rede, precisa apenas de uma tabela com informações sobre o SRLG-ID e o comprimento de onda já usado na entidade de risco por este SRLG identificado. Portanto, não há modificação nas trocas de mensagens, apenas um incremento das mensagens já existentes pois quando as mensagens de manutenção e atualização forem enviadas pelo LMP, estas mesmas mensagens farão a manutenção e a atualização da tabela SRLG_ $\lambda$ em cada nó.

O conjunto de rótulos é inserido na mensagem Path (mensagem de pedido de rótulo) e assim não altera a sinalização e o funcionamento do LMP, apenas estende as mensagens destes. A mensagem Resv (mensagem de atribuição de rótulos) permanece sem alterações. No entanto, a mensagem Path e a mensagem Resv possuem suas próprias máquinas de estado finito de comunicação.

Para melhor entender a máquina de estado finito de comunicação, segue a especificação mais detalhada do protocolo de sinalização durante a atribuição de comprimento de onda (Figura 6.1). A definição dos estados definidos na especificação é como segue:

1. SRLG é estabelecido para todos os enlaces da rede antes da rede ser colocada em funcionamento

2. Conexão é solicitada ao nó de ingresso

3. Rota da conexão é escolhida de acordo com nó de origem e destino

4. O SRLG-caminho é conhecido

5. Mensagem Path (pedido de rótulo) é enviada pelo nó upstream para o nó downstream

6. Nó downstream verifica se a codificação LSP utilizada e tipo de comutação são suportados

7. Cada nó gera um conjunto de rótulos de saída com base na informação SRLG 
8. Verifica-se se o conjunto de rótulos de saída gerado é vazio ou não

9. Mensagem Path que transporta o conjunto de rótulos é recebida pelo nó de egresso

10. Verifica-se se o conjunto de rótulos final é vazio ou não

11. Tenta-se selecionar um rótulo (comprimento de onda) a partir do conjunto de rótulos

12. Mensagem Resv (atribuição de rótulos) é enviada do nó downstream para o nó upstream, até o nó de ingresso

13. Verifica-se se o valor do rótulo transportado pela mensagem Resv é aceitável ou não

14. Atribuição do comprimento de onda é realizada com sucesso e as tabelas de SRLG em cada nó são atualizadas

15. Alguma falha ocorreu e a conexão não foi estabelecida

Definição das mensagens utilizadas na especificação:

A. Todos os possíveis caminhos foram utilizados sem sucesso

B. A atribuição de rótulos para o caminho atual fracassou. Um novo caminho é estabelecido na tentativa de atender ao pedido de conexão

C. Mensagem PathErr é enviada com a indicação "problema de roteamento / codificação não suportada" ou "problema de roteamento / tipo de comutação"

D. Mensagem PathErr é enviada com a indicação "problema de roteamento / conjunto de rótulos"

E. Se todas as opções de caminho foram testadas sem sucesso e o conjunto de rótulos continua vazio, uma mensagem PathErr é enviada com a indicação "problema de roteamento / conjunto de rótulos"

F. Nó é incapaz de escolher um rótulo ou existe um problema em analisar o conjunto de rótulos. Mensagem PathErr é enviada com a indicação "problema de roteamento conjunto de rótulos" 
G. Se o valor do comprimento de onda não for aceitável por algum nó, uma mensagem ResvErr é enviada com a indicação "problema de roteamento / falha na alocação de rótulos".

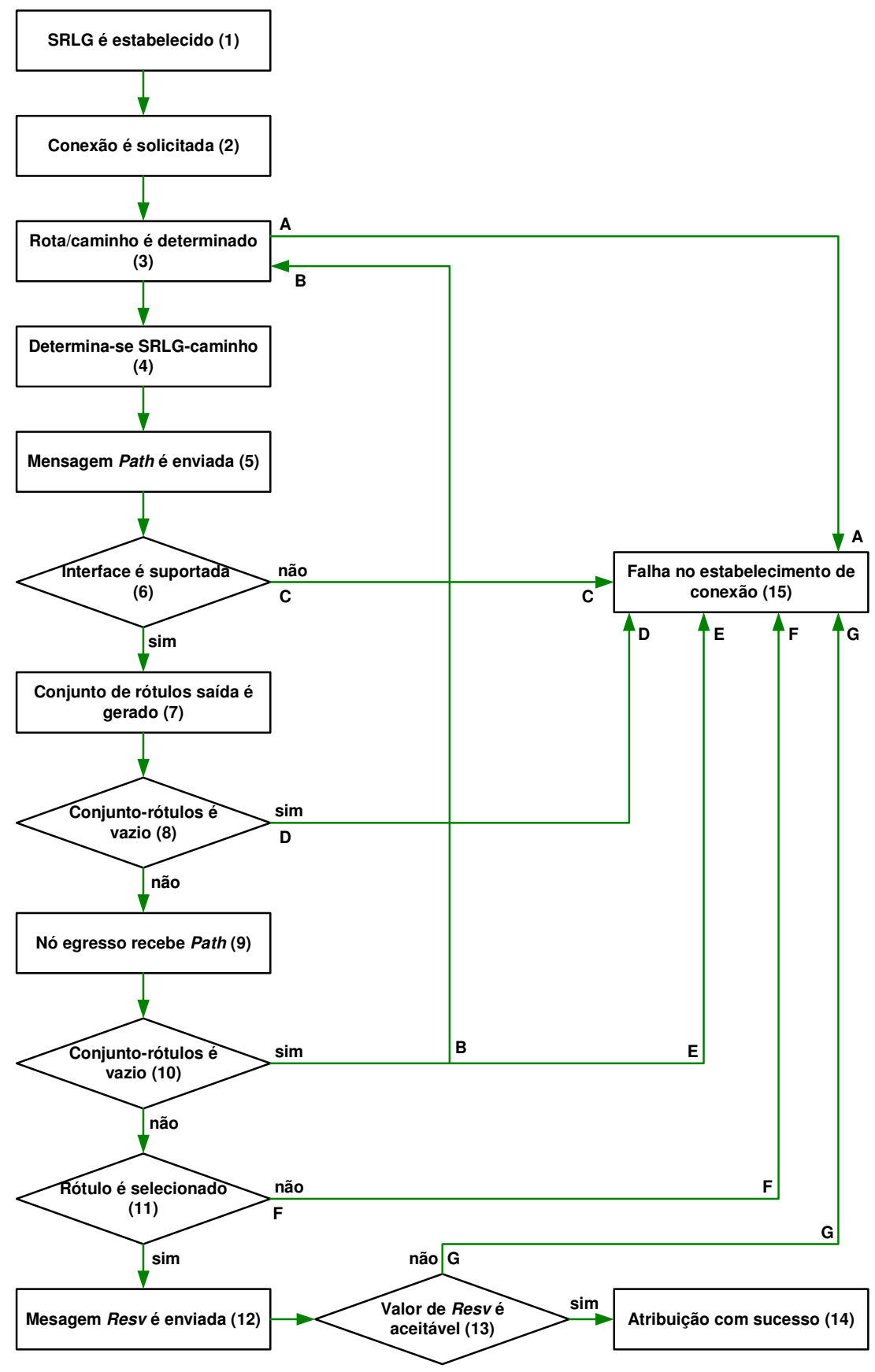

Figura 6.1 - Definição do protocolo de sinalização para atribuição de comprimento de onda. 
Com base na especificação apresentada na Figura 6.1, determina-se a máquina de estado finito de comunicação do protocolo de sinalização para atribuição de rótulos. A Figura 6.2 apresenta a máquina de estado finita de comunicação (MEFC) entre um nó upstream (a esquerda na figura) e um nó downstream (a direita na figura) situado no núcleo da rede. A Figura 6.4 apresenta a máquina de estado finita de comunicação (MEFC) entre um nó upstream (a esquerda na figura) e um nó downstream (a direita na figura) considerado como nó de egresso. Essas duas situações foram consideradas separadamente para uma melhor compreensão da troca de mensagens em cada uma das situações.
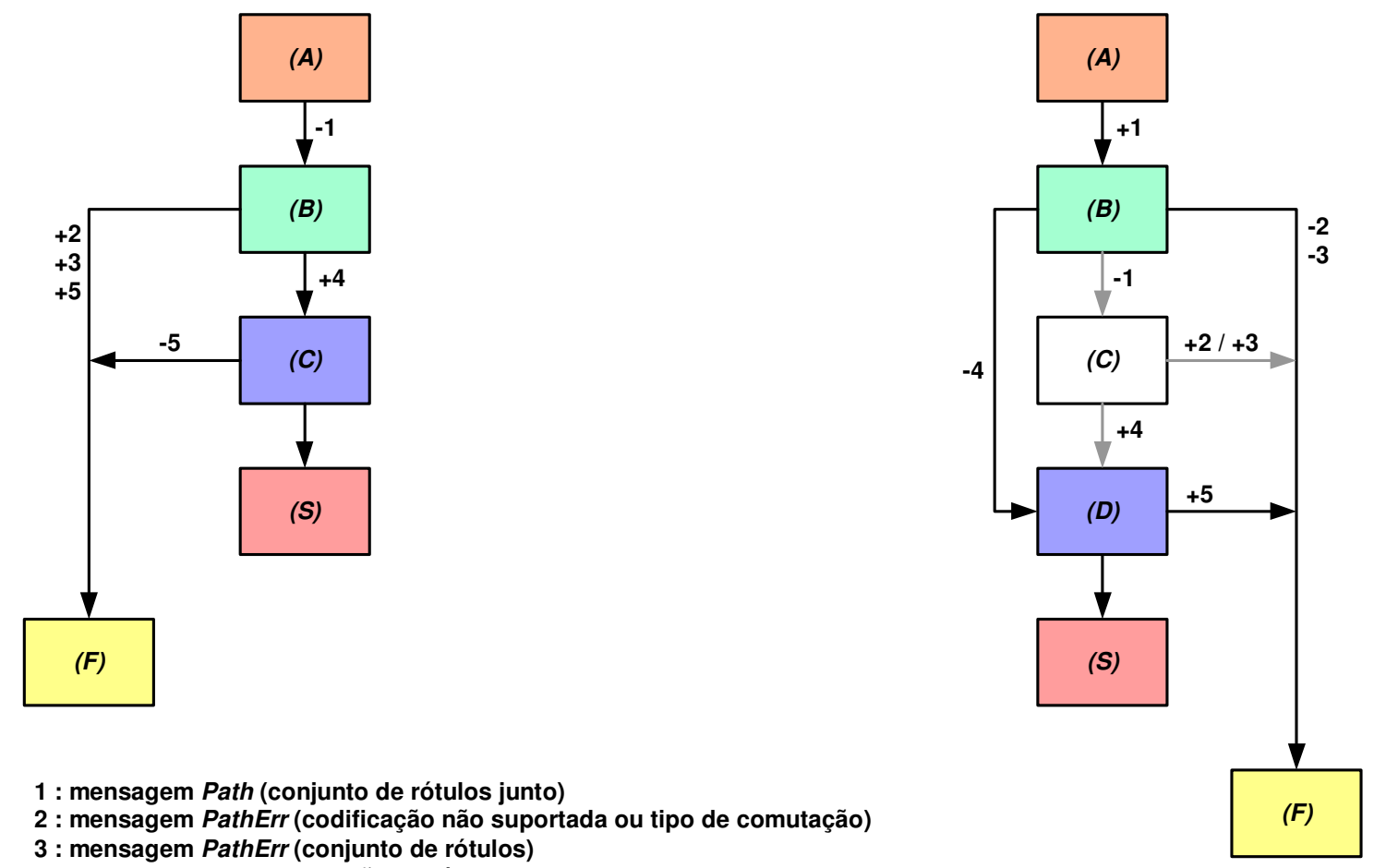

1 : mensagem Path (conjunto de rótulos junto)

2 : mensagem PathErr (codificação não suportada ou tipo de comutação)

3 : mensagem PathErr (conjunto de rótulos)

4 : mensagem Resv (atribuição de rótulos)

5 : mensagem ResvErr

- comunicação entre upstream e downstream

comunicação do downstream com seu downstream

Figura 6.2 - Máquina de estado finito de comunicação entre nó upstream e um nó downstream em um ponto intermediário da rede. 
Com base na máquina de estado finita de comunicação apresentada na Figura 6.2, é possível aplicar a técnica de análise lógica denominada análise com progresso justo e verificar se a parte do protocolo de sinalização relacionada com atribuição de rótulos possui ou não erros de progresso.

O resultado desta análise é a construção do grafo de alcançabilidade justa, como mostra a Figura 6.3. De acordo com a grafo e com base nas definições de erros de progresso apresentadas anteriormente, nota-se que a sinalização relacionada a atribuição de rótulos não apresenta deadlocks, recepções não especificadas nem mesmo, interações não-executadas, livelocks e overflow.

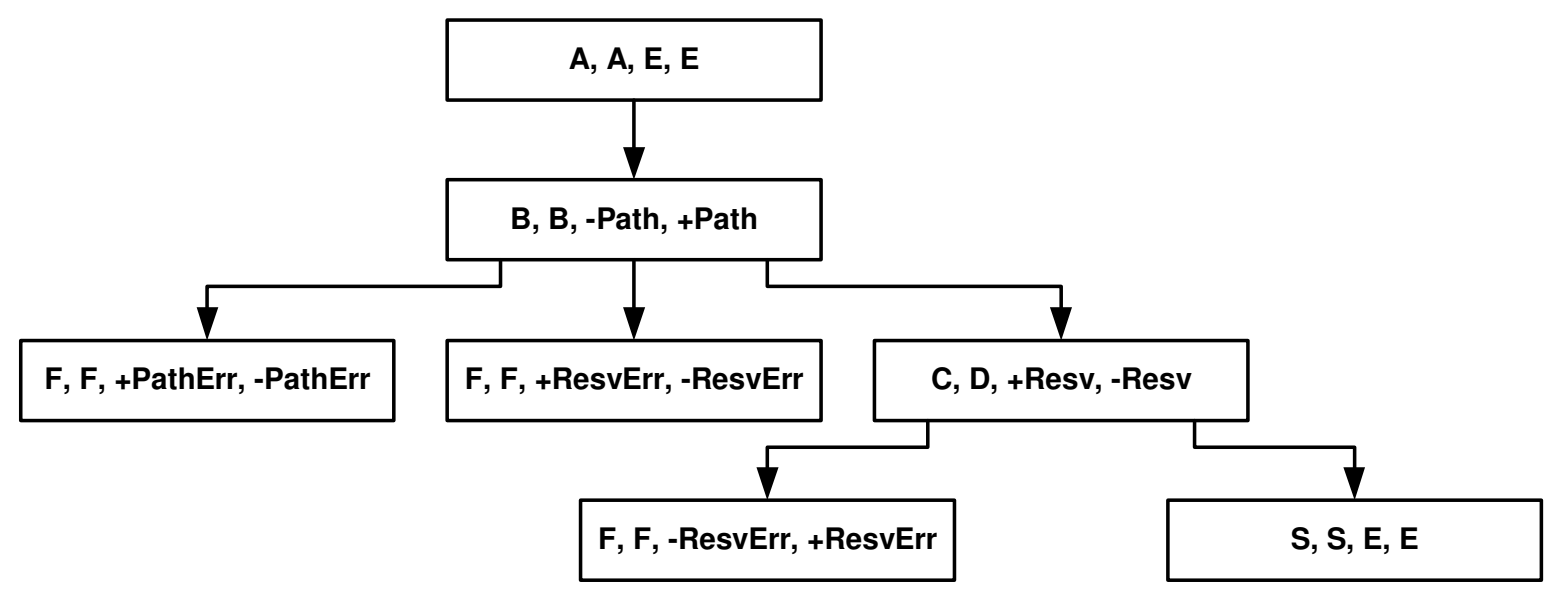

Figura 6.3 - Análise justa da MEFC da sinalização de atribuição de rótulos em um ponto intermediário da rede.

Com base na máquina de estado finita de comunicação apresentada na Figura 6.4, é possível aplicar a técnica de análise lógica com progresso justo e verificar se a parte do protocolo de sinalização relacionada com atribuição de rótulos possui ou não erros de progresso.

O resultado desta análise é a construção do grafo de alcançabilidade justa, como mostra a Figura 6.5. De acordo com a grafo e com base nas definições de erros de progresso 
apresentadas anteriormente, nota-se que a sinalização relacionada a atribuição de rótulos não apresenta erros de progresso.
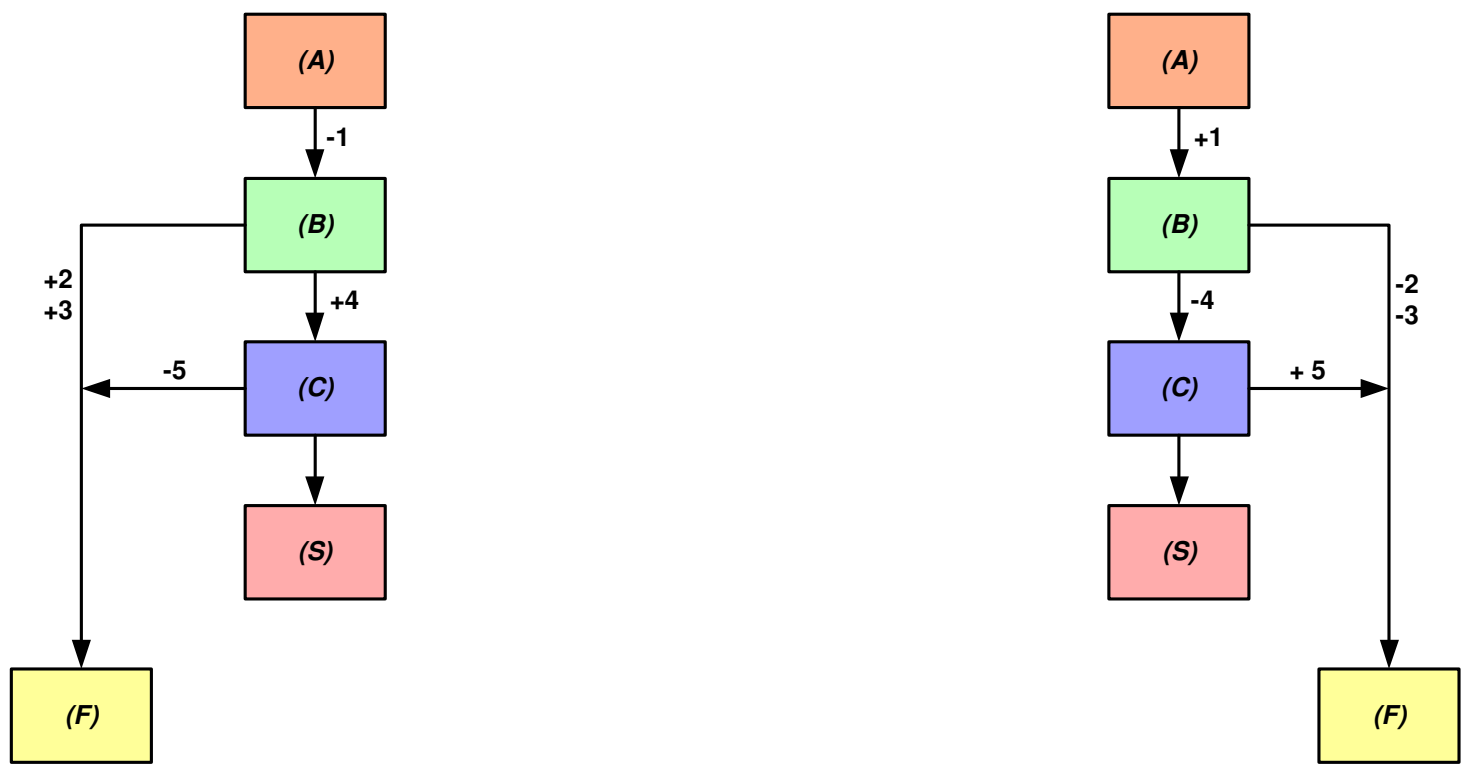

1 : mensagem Path (conjunto de rótulos junto)

2 : mensagem PathErr (codificação não suportada ou tipo de comutação)

3 : mensagem PathErr (conjunto de rótulos)

4 : mensagem Resv (atribuição de rótulos)

5 : mensagem ResvErr

comunicação entre upstream e downstream

Figura 6.4 - Máquina de estado finito de comunicação entre nó upstream e um nó downstream considerado nó de egresso da rede.

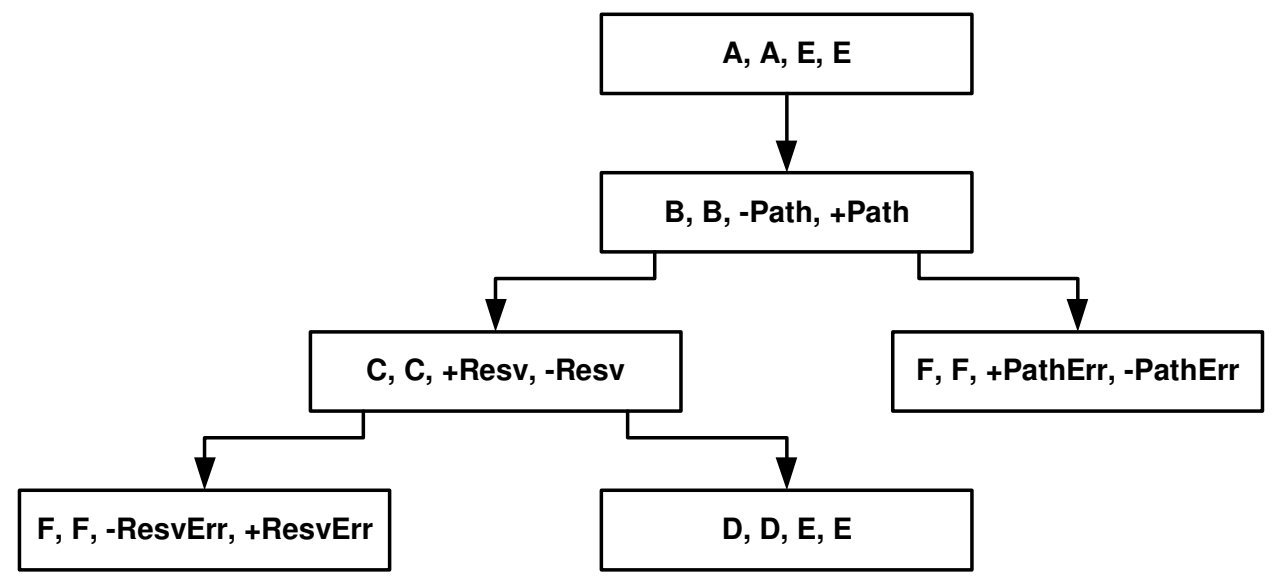

Figura 6.5 - Análise justa da MEFC da sinalização de atribuição de rótulos no ponto de egresso da rede. 


\subsection{SIMULAÇÃO}

A análise lógica não é suficiente para provar a eficiência do algoritmo de atribuição de comprimentos de onda proposto. Para esta finalidade é necessário fazer uso de um simulador para verificar se a proposta realmente consegue atender o problema RWA e diminuir a taxa de probabilidade de bloqueio de conexão em um ambiente GMPLS-WDM.

Em uma simulação, as avaliações de desempenho são realizadas com modelos do sistema e não com o sistema. Dessa forma, não há a necessidade de construção de uma rede real baseada nas tecnologias GMPLS e WDM para avaliar as heurísticas de alocação de comprimento de onda apresentadas anteriormente. Neste caso é também possível exercitar diferentes cenários e analisar a questão de escalabilidade o que é impraticável em sistemas reais.

As simulações são realizadas utilizando cenários GMPLS-WDM que apresentam o problema de trap e cenários que não apresentam este problema. Estes cenários de simulação também devem ser diferenciados pela quantidade de comprimentos de onda, ou seja, quantidade de rótulos disponibilizados e que podem ser utilizados em cada enlace óptico.

A eficiência do algoritmo de atribuição de rótulos proposto é analisada através dessas simulações. Os resultados são comparados entre as redes GMPLS-WDM que utilizam as heurísticas de atribuição de comprimento de onda apresentadas pela literatura e as redes GMPLS-WDM com SRLG para atribuição de rótulos, com o objetivo de comprovar a maior eficiência do algoritmo proposto. 
Todas as avaliações foram feitas com base no simulador NS [NS] e Matlab. O núcleo do simulador NS e os toolkits do Matlab foram mantidos sendo que adaptações necessárias foram desenvolvidas a fim de reproduzir o funcionamento de cada um dos algoritmos de atribuição de comprimento de onda a serem avaliados.

\subsubsection{Descrição dos Simuladores}

Para implementar os cenários GMPLS-WDM, foram utilizados dois programas simuladores.

O primeiro foi o simulador de eventos discretos da Universidade de Berkeley denominado UCB/LBNL Network Simulator, versão NS-2.1b6 [NS]. Este programa simulador é intensamente utilizado para estudos de protocolos de rede e também para fins educativos. $\mathrm{O}$ NS2 suporta uma linguagem de script bem definida para a implementação de topologias de redes e outros parâmetros de simulação. Além disso, o NS2 gera saídas na forma de arquivos trace, bem como saídas no terminal e oferece suporte a uma ferramenta de animação denominada Network Animator - NAM. O NAM utiliza os arquivos trace gerados pelo NS2 para apresentar a visualização da simulação.

O NS2 é de domínio público e seu código fonte está disponível livremente. O código é escrito na linguagem de programação $\mathrm{C}++$ e muitas extensões do programa simulador são feitas em uma linguagem denominada OTcl.

Juntamente com o NS2 foi utilizada outra ferramenta de simulação denominada Optical WDM Network Simulator - OWNS [OWNS]. Este programa simulador foi implementado como uma extensão do NS e desenvolvido em sistema Red Hat Linux, com base no próprio NS2.1b6. O 
principal propósito do Dawn Networking Research Lab, da University of Maryland, foi desenvolver um programa simulador que permitisse a simulação de várias características e aplicações ópticas sem ter a necessidade de construir uma rede real baseada na tecnologia WDM.

Além do simulador NS, foi utilizado o Matlab, ferramenta matemática juntamente com o toolkit WDM desenvolvido por Przemyslaw Pawelczak.

Ambos os simuladores, NS e Matlab, apresentam resultados semelhantes durante as simulações e por essa razão, na apresentação dos resultados não é mencionado o simulador específico.

A intenção ao se implementar um simulador para a rede GMPLS-WDM é permitir uma simplificação da análise deste estudo de avaliação de desempenho. A simulação proporciona análises rápidas e reduz os detalhes de implementação a serem manipulados.

\subsubsection{Topologias Simuladas, Avaliação de Desempenho, Apresentação e Análise dos Resultados}

Com o objetivo de avaliar e comparar os principais algoritmos de atribuição de comprimento de onda com relação a probabilidade de bloqueio de conexão, construiu-se diferentes modelos de simulação baseados em backbones já implementados e/ou conhecidos (de forma que os resultados pudessem ajudar na validação dos simuladores e dos resultados obtidos). Estes backbones sofreram algumas modificações de forma que pudessem ser simulados dentro dos limites permitidos pelos programas simuladores utilizados, i.e., um dos simuladores não 
oferece suporte a um número elevado de nós (simulador NS). Estas modificações foram realizadas de forma a manter as características da rede nos modelos simulados.

Nesta seção, é também apresentada a probabilidade de bloqueio de conexão de cada cenário simulado quando as heurísticas de atribuição de comprimento de onda são implementadas.

As redes GMPLS-WDM consideradas para a realização deste estudo são apresentadas nas Figuras 6.6, 6.13, 6.18 e 6.23. O núcleo dos backbones simulados são formados por comutadores ópticos interligados que utilizam a tecnologia WDM e GMPLS originando assim uma rede GMPLS-WDM. Como o fluxo de dados é bidirecional em todos os enlaces, todos os nós podem se comportar como comutadores de origem e destino, dependendo da direção deste fluxo.

Abaixo, apresenta-se uma descrição de alguns parâmetros e considerações em comum, relacionados a todos os cenários simulados:

- Pedidos de conexão: distribuição de Poisson

- Duração do serviço: distribuição exponencial

- Tempo de retransmissão: $1,5 \mathrm{~ms}$

- Protocolo de roteamento: roteamento adaptativo

- Carga de tráfego representada em Erlangs ${ }^{15}$

- RWA dinâmico

- Parâmetro de medida de desempenho: probabilidade de bloqueio de conexão

- Situação ótima: situação ideal utilizada para comparações e representa uma rede com conversão total de comprimento de onda em um ambiente RWA dinâmico ${ }^{16}$

\footnotetext{
${ }^{15}$ Erlang é uma unidade de medida de tráfego em telecomunicação. Precisamente um Erlang representa o uso contínuo de um caminho. Erlang = 3 representa 3 chamadas simultâneas e cada uma com duração de uma hora, ou 6 chamadas, cada uma com duração de 30 minutos, ou 180 chamadas cada uma com duração de 1 minuto.
} 
O interesse está em avaliar o desempenho de cada algoritmo de atribuição de comprimento de onda, mencionados no Capítulo III, aplicados em um ambiente GMPLS-WDM, em termos da probabilidade de bloqueio de conexões durante o encaminhamento de dados. Sendo que a probabilidade de bloqueio de conexão é definida como o número de pedidos de conexão bloqueados durante todo o tempo de simulação dividido pelo número total de pedidos de conexão.

O cenário ótimo considerado é das redes com conversão total de comprimentos de onda, pois utilizam de forma eficiente os comprimentos de onda resolvendo conflitos durante o estabelecimento de conexões. Portanto, o mais próximo que se conseguir chegar dos resultados apresentados pelo cenário ótimo, melhor será a eficiência do algoritmo de atribuição de comprimento de onda para as redes com restrição de continuidade de comprimento de onda.

\subsubsection{Topologia com 6 Nós}

Apresenta-se abaixo as descrições específicas da topologia de 6-nós (Figura 6.6):

- Número de nós: 6

- Número de enlaces: 8

- Número de comprimentos de onda por enlace (W): 4 - 16

- Número de fibras (M): 1 - 4

- Número de enlaces com trap: 4

\footnotetext{
${ }^{16}$ Situação ideal considera RWA dinâmico, pois o RWA estático apresenta resultados melhores em termos de desempenho, porém são resultados que não representam a realidade (que utiliza RWA dinâmico).
} 


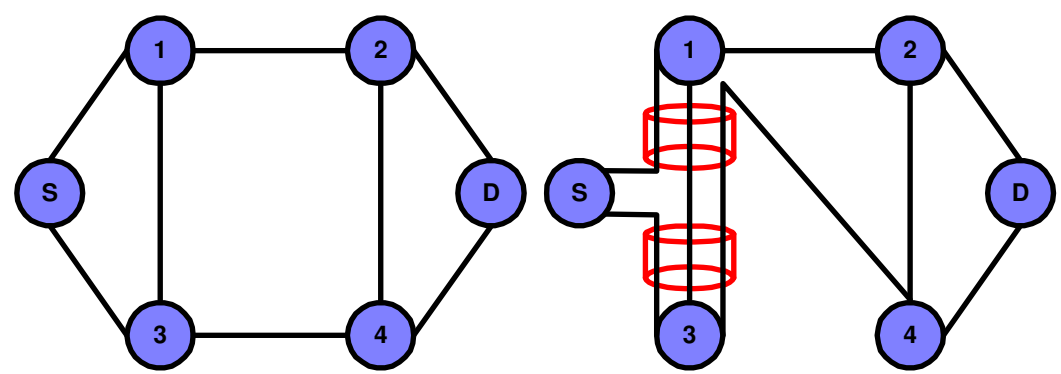

Figura 6.6 - Topologia com 6 nós.

Com base nos resultados apresentados pela simulação na Topologia de 6 nós, é possível comparar as heurísticas propostas pela literatura e a heurística proposta neste estudo. Variações no número de fibras e número de comprimentos de onda disponíveis são realizadas para que seja possível analisar a interferência do número de fibras e número de comprimentos de onda no desempenho de cada algoritmo de atribuição de comprimento de onda.

De acordo com a Figura 6.7(a) pode-se afirmar que as heurísticas de atribuição de comprimento de onda apresentam desempenho semelhante na rede com 6-nós sendo que cada enlace possui 1 fibra (M) e 16 comprimentos de onda disponíveis entre os nós (W). Verificase que o algoritmo DRCL possui desempenho superior de aproximadamente $10 \%$ se comparado ao MU, que apresenta o segundo melhor resultado, e 15\% se comparado ao LU, que apresenta a maior probabilidade de bloqueio. Este melhor desempenho do DRCL é mais significativo quando a carga de tráfego é maior.

Os algoritmos MU e FF possuem resultados praticamente iguais em termos de probabilidade de bloqueio de conexão, como apresenta a Figura 6.7(a). O algoritmo RD possui probabilidade de bloqueio de conexão aproximadamente $10 \%$ superior se comparado ao algoritmo FF. 
Observando a Figura 6.7(b), pode-se afirmar que para este cenário, $M=1 \mathrm{e} \mathrm{W}=16$ sem problema de trap, o algoritmo proposto possui desempenho equivalente ao algoritmo DRCL, como era esperado.

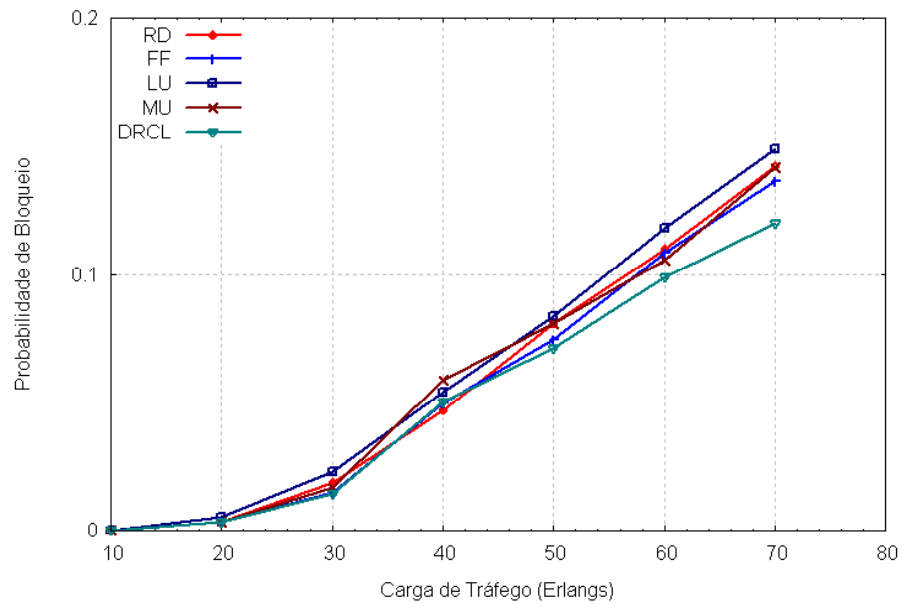

(a)

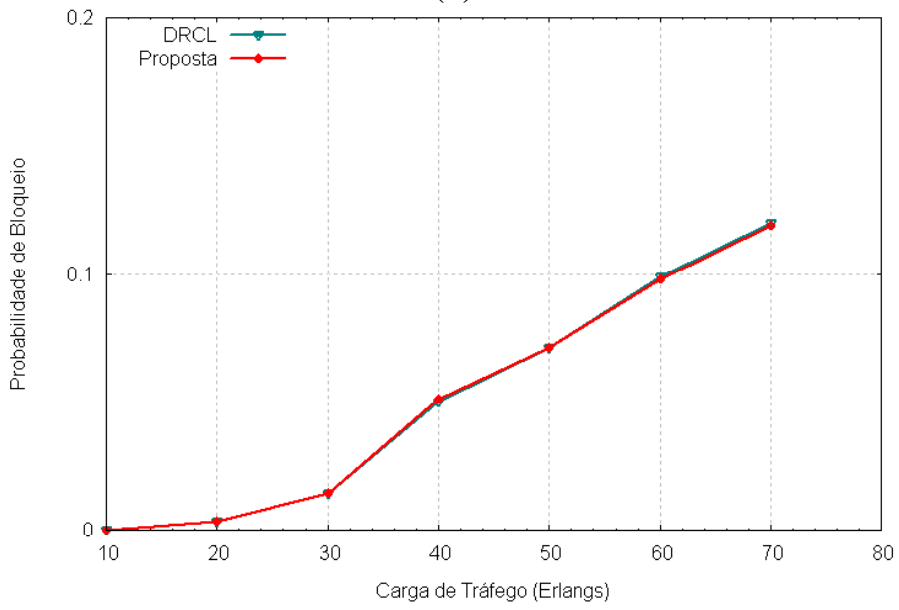

(b)

Figura 6.7 - Topologia de 6 nós com $M=1$ e $W=16$ comprimentos de onda sem problema de Traps.

Analisando a Figura 6.8(a), nota-se que as heurísticas de alocação de comprimento de onda possuem resultados próximos. A diferença, não significativa, entre cada desempenho é verificado de forma mais clara quando a carga de tráfego é maior (valor acima de 50 Erlangs). 
A Figura 6.8(b) mostra a equivalência entre os algoritmos DRCL e proposto, como verificado no cenário anterior.

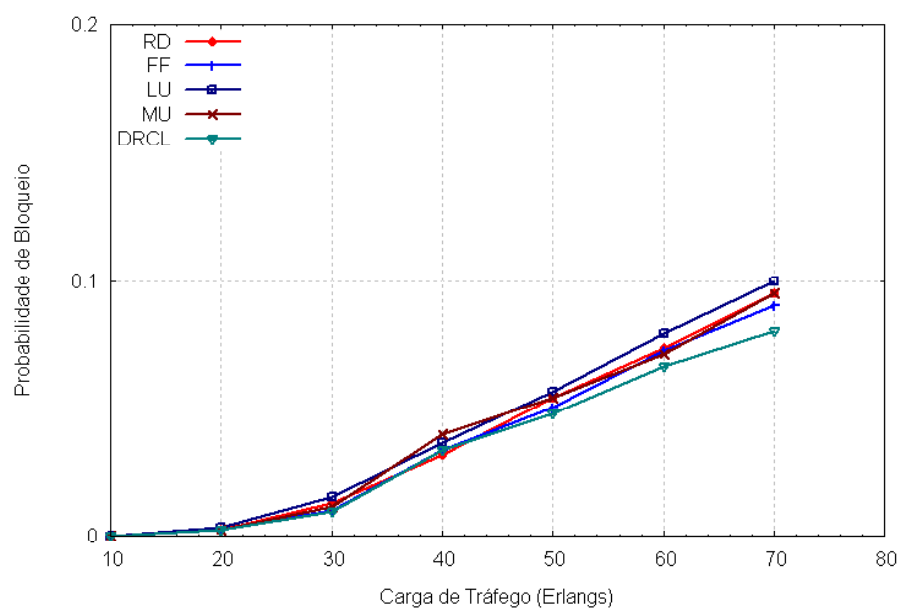

(a)

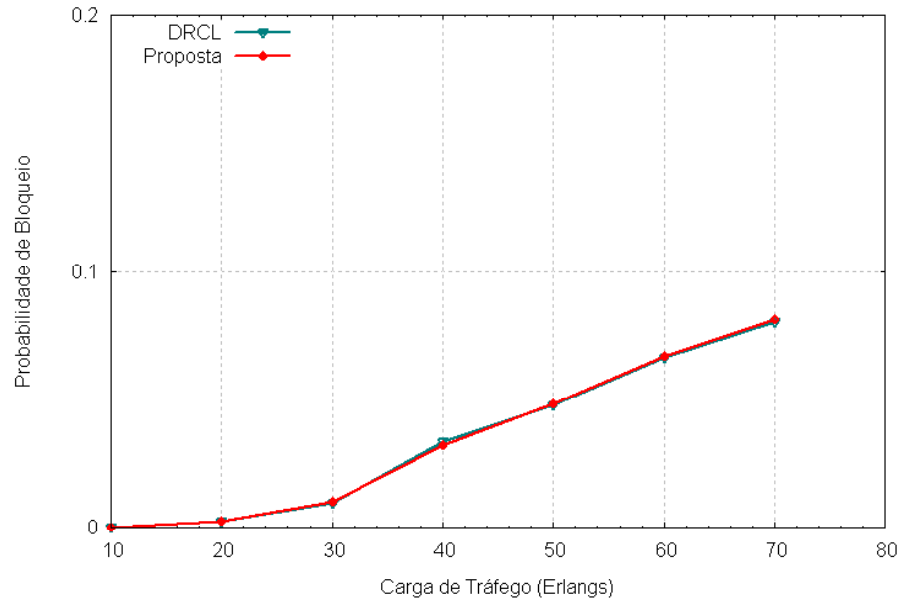

(b)

Figura 6.8 - Topologia de 6 nós com $\mathrm{M}=2$ e $\mathrm{W}=8$ comprimentos de onda sem problema de Traps.

O cenário que possui um número maior de fibras entre os nós (Figura 6.8) apresenta resultado aproximadamente $45 \%$ melhor se comparado ao cenário com $\mathrm{M}=1 \mathrm{e} \mathrm{W}=16$ (Figura 6.7). Assim, mesmo o número de combinações entre fibras e comprimentos de onda sendo iguais para ambos os cenários, a rede com $\mathrm{M}=2$ apresenta melhor desempenho se comparado a rede com $\mathrm{M}=1$. Pode-se concluir que o número de fibras em cada enlace, para este cenário, 
interfere no desempenho da rede, mas há também a dependência pelo número de comprimentos de onda disponíveis.

De acordo com a Figura 6.9(a), a semelhança entre as heurísticas é mantida como observado nos cenários anteriores.

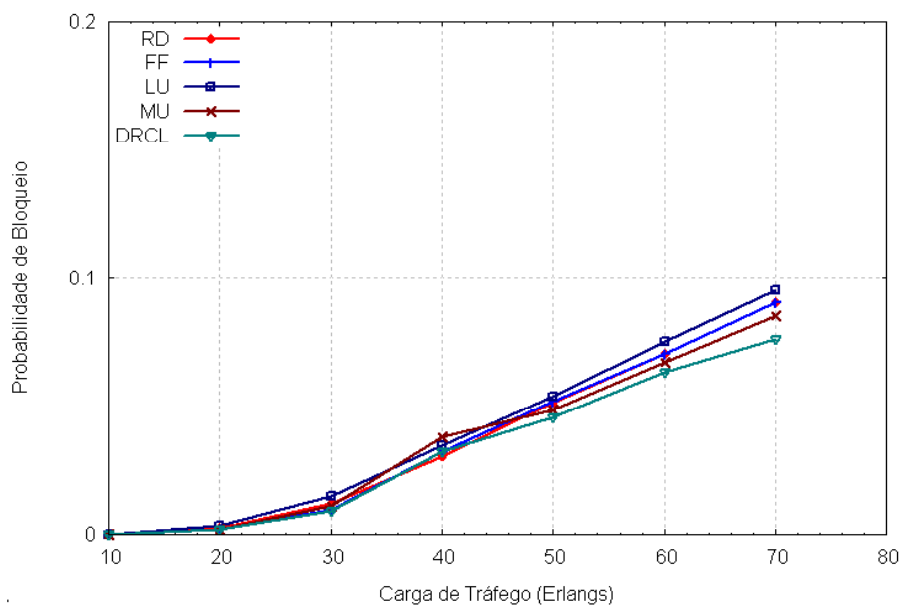

(a)

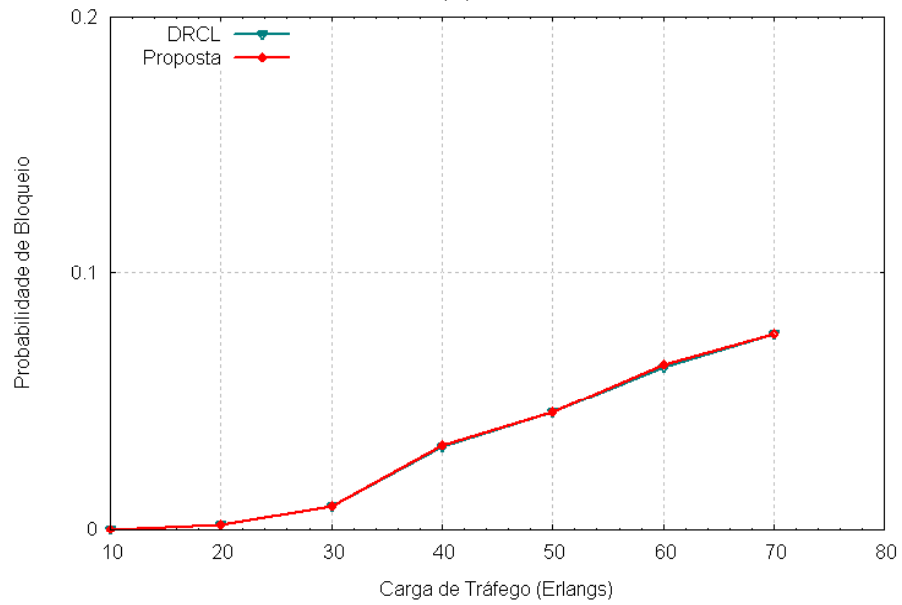

(b)

Figura 6.9 - Topologia de 6 nós com $\mathrm{M}=4$ e $\mathrm{W}=4$ comprimentos de onda sem problema de Traps.

Nota-se que com o aumento no número de fibras em cada enlace $(\mathrm{M}=4)$ o desempenho melhorou em aproximadamente 5\% se comparado ao cenário com 2 fibras em cada enlace (Figura 6.8). Verifica-se que o desempenho não apresenta uma diferença significativa se 
comparado ao desempenho do cenário anterior $(\mathrm{M}=2 \mathrm{~W}=8)$. Logo, o número de fibras entre os enlaces interfere na probabilidade de bloqueio, mas esse aumento no número de fibras pode chegar a um limite onde a sua alteração não causa interferência brusca na probabilidade de bloqueio.

Comparando o cenário $\mathrm{M}=1$ e $\mathrm{W}=16$ sem o problema de trap (Figura 6.7) e o cenário $\mathrm{M}=$ $1 \mathrm{e} \mathrm{W}=16$ com problema de traps (Figura 6.10), nota-se que o desempenho das heurísticas de atribuição de comprimento de onda é menor no cenário com traps pois as heurísticas apresentadas na literatura não lidam com o problema de traps gerando assim um número maior de bloqueio de conexão. O desempenho é aproximadamente $48 \%$ menor se comparado ao desempenho no cenário que não apresenta problema de trap. De qualquer forma, as heurísticas, comparadas entre si, apresentam desempenho semelhante como nos cenários já analisados.

A Figura 6.10(b) mostra o desempenho superior do algoritmo proposto se comparado aos demais principalmente quando a carga de tráfego é superior a 40 Erlangs. Isso se deve ao fato do algoritmo proposto reconhecer e lidar com o problema de traps, o que gera um menor bloqueio de conexões. O algoritmo proposto possui desempenho superior em aproximadamente $35 \%$ se comparado ao algoritmo DRCL que é o algoritmo apresentado pela literatura com melhor desempenho.

Observando a Figura 6.11(a), percebe-se que as heurísticas RD, FF, LU, MU e DRCL apresentam uma visível diferença quando a carga de tráfego é maior, ou seja, acima de 50 Erlangs. Comparando a Figura 6.8 e 6.11, nota-se que o desempenho dos algoritmos de 
atribuição de comprimento de onda no cenário que apresenta traps é aproximadamente 50$55 \%$ menor que do cenário sem o problema de traps.

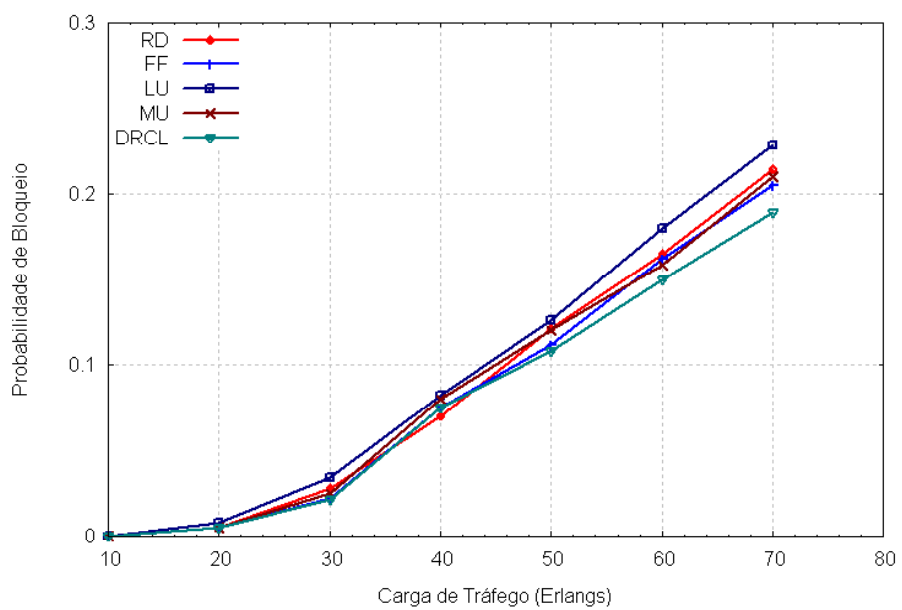

(a)

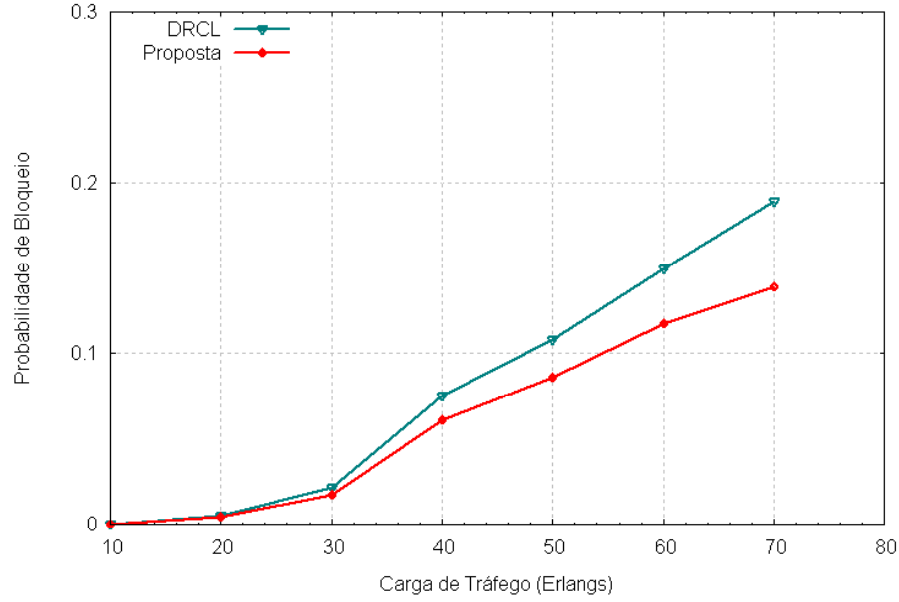

(b)

Figura 6.10 - Topologia de 6 nós com $\mathrm{M}=1$ e $\mathrm{W}=16$ comprimentos de onda com problema de Traps.

A figura 6.11(b) mostra a diferença entre o algoritmo proposto e o algoritmo DRCL. O algoritmo proposto apresenta uma menor probabilidade de bloqueio de conexão principalmente quando a carga de tráfego está acima de 40 Erlangs. O desempenho da proposta é maior, pois lida com o problema de traps através do uso do conceito SRLG. 


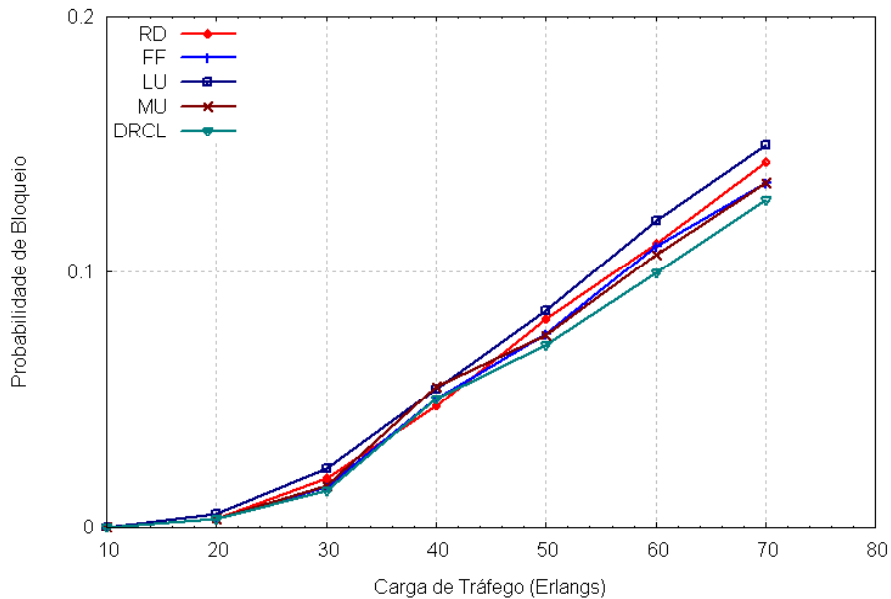

(a)

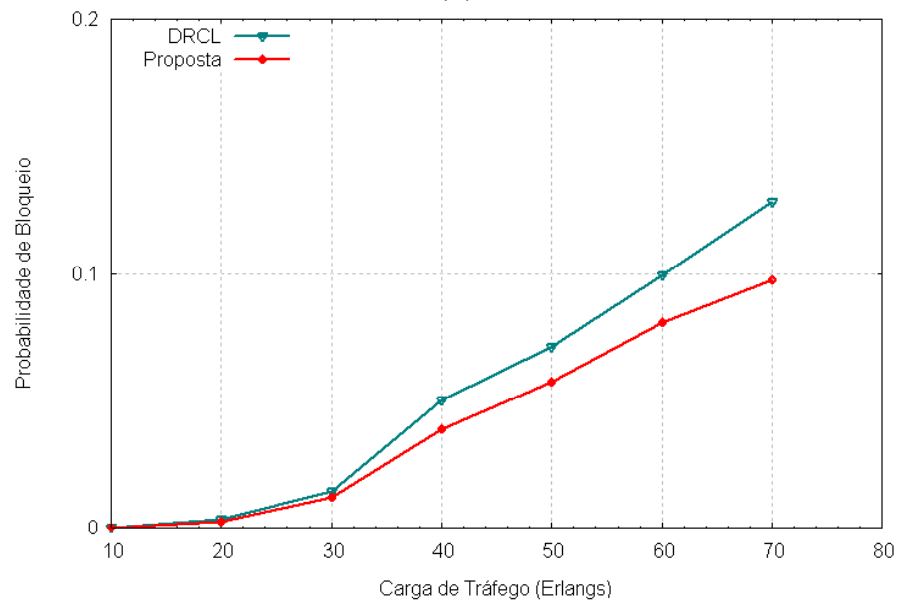

(b)

Figura 6.11 - Topologia de 6 nós com $\mathrm{M}=2$ e $\mathrm{W}=8$ comprimentos de onda com problema de Traps.

Analisando a Figura 6.12, percebe-se que o cenário $\mathrm{M}=4$ e $\mathrm{W}=4$, mesmo com o problema de traps, continua a manter o desempenho superior de aproximadamente $5 \%$ se comparado ao cenário $\mathrm{M}=2 \mathrm{e} \mathrm{W}=8$ com problema de traps. $\mathrm{O}$ mesmo acontece com estes cenários quando não apresentam o problema de traps, pois a diferença no resultado de desempenho se mantém em $5 \%$.

De acordo com a Figura 6.12(b), o algoritmo proposto possui uma menor probabilidade de bloqueio quando a carga de tráfego está acima de 40 Erlangs. Isso se deve ao fato da 
eficiência do algoritmo ser verificada quando o número de pedidos de recurso é maior e assim, com a melhor eficiência do algoritmo proposto, o desempenho é melhor.

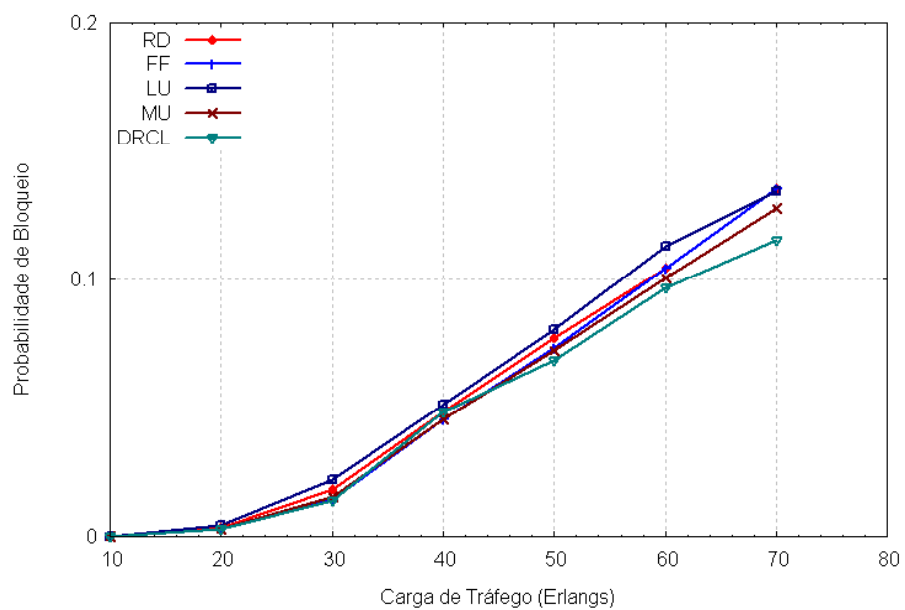

(a)

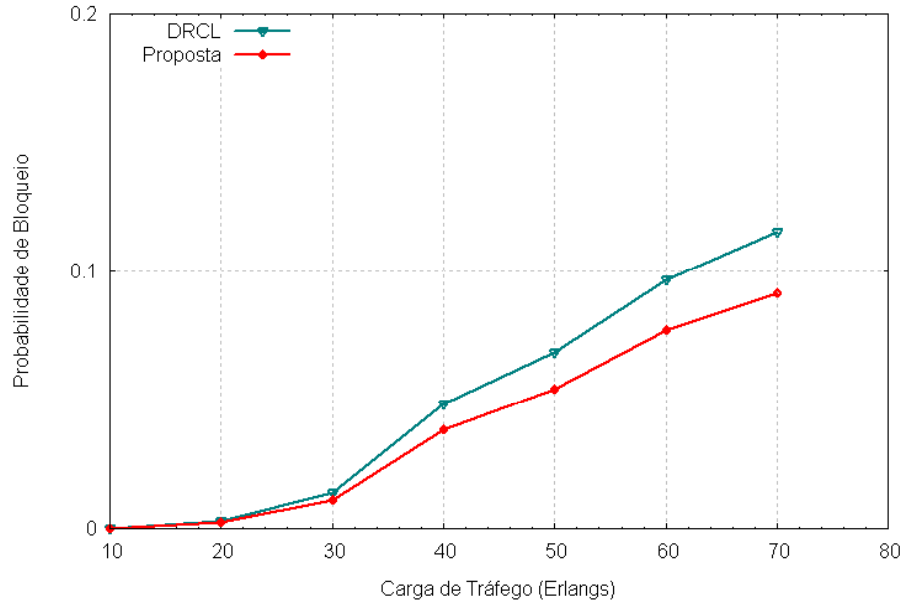

(b)

Figura 6.12 - Topologia de 6 nós com $\mathrm{M}=4$ e $\mathrm{W}=4$ comprimentos de onda com problema de Traps.

\subsubsection{Topologia NSFNET}

Apresenta-se abaixo as descrições específicas da topologia NSFNET (National Science Foundation Network) (Figura 6.13):

- Número de nós: 14

- Número de enlaces: 21 
- Número de comprimentos de onda por enlace (W): 32 e 40

- Número de fibras (M): 1

- Número de enlaces com trap: 8

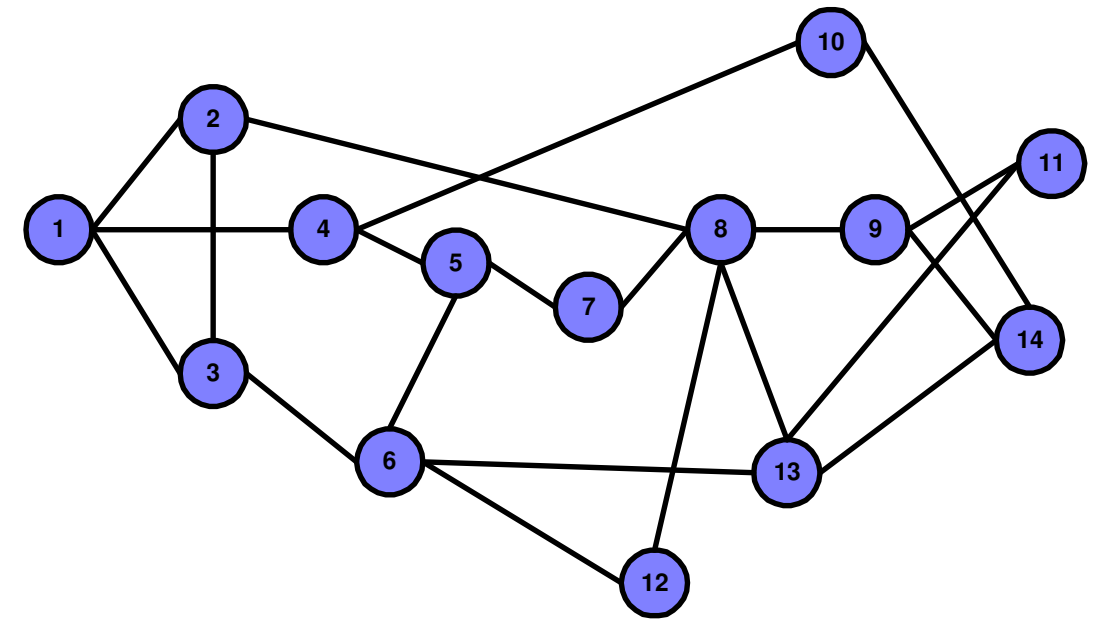

Figura 6.13 - Topologia NSFNET.

Através da simulação realizada com o cenário NSFNET é possível comparar as heurísticas de atribuição de comprimento de onda propostas pela literatura, bem como comparar o algoritmo proposto com essas heurísticas e também com a situação ideal.

Observando a Figura 6.14(a), pode-se afirmar que as heurísticas de atribuição de comprimento de onda apresentam desempenho semelhante na rede com 32 comprimentos de onda. Verifica-se também, de forma mais detalhada, que o algoritmo DRCL na Figura 6.14(a) possui desempenho superior em aproximadamente $20 \%$ quando comparado ao algoritmo MU que apresenta o segundo melhor desempenho. Os algoritmos MU e FF apresentam resultados praticamente idênticos em termos de probabilidade de bloqueio, verificado e avaliado na Figura 6.14(a) em referência. O algoritmo RD apresenta um desempenho inferior ao FF em aproximadamente 10\%. O algoritmo LU apresenta uma probabilidade de bloqueio maior, principalmente quando a rede apresenta uma carga de tráfego mais elevada. 


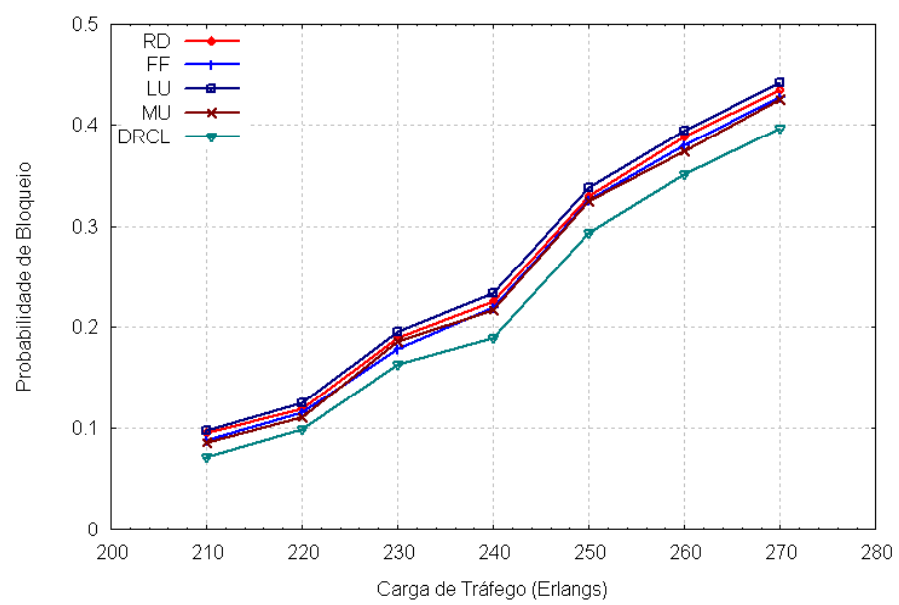

(a)

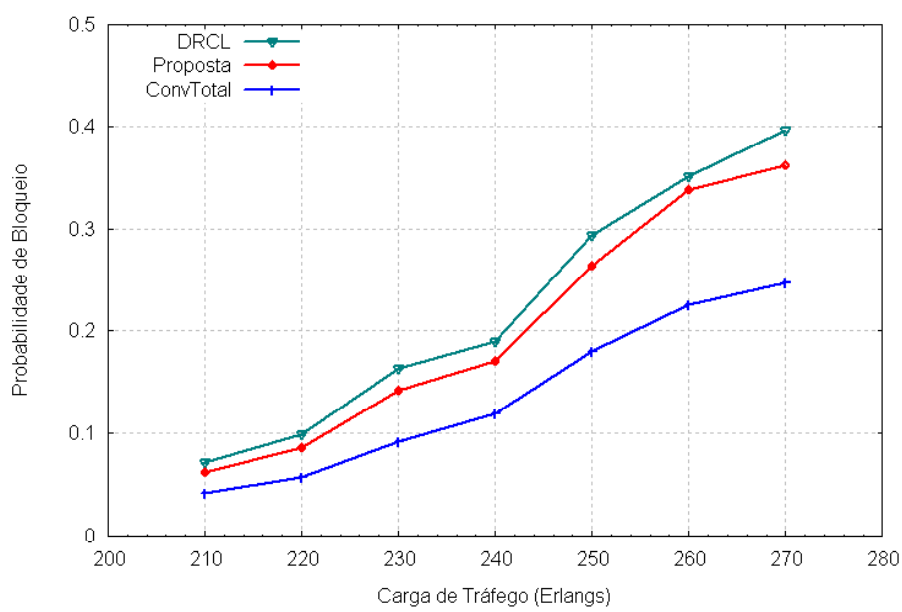

(b)

Figura 6.14 - Topologia NSFNET com 32 comprimentos de onda sem problema de Traps.

Avaliando por comparação o algoritmo DRCL (algoritmo que apresentou o melhor desempenho proposto na leitura), o algoritmo proposto e a situação ótima (quando a rede apresenta conversão total de comprimento de onda), nota-se que tanto o DRCL quanto o algoritmo proposto demonstraram desempenhos semelhantes neste cenário e próximos à situação ideal, sendo que o algoritmo proposto, dependendo da carga de tráfego da rede, apresenta um desempenho aproximadamente $15 \%$ melhor que DRCL, conforme se observa na Figura 6.14(b). 
A Figura 6.15(a) confirma os resultados do cenário anterior (Figura 6.14(a)), pois as heurísticas apresentadas na literatura possuem desempenhos muito próximos, porém aproximadamente $25 \%$ superior que no cenário com 32 comprimentos de onda devido ao maior número de comprimentos de onda disponíveis na rede, pois assim um número maior de conexões pode ser atendido. O mesmo acontece com os resultados da Figura 6.15(b), onde verifica-se que o algoritmo proposto e o DRCL têm resultados próximos, quando se trata de probabilidade de bloqueio, porém com melhora significativa ao ser comparado com o cenário de 32 comprimentos de onda.

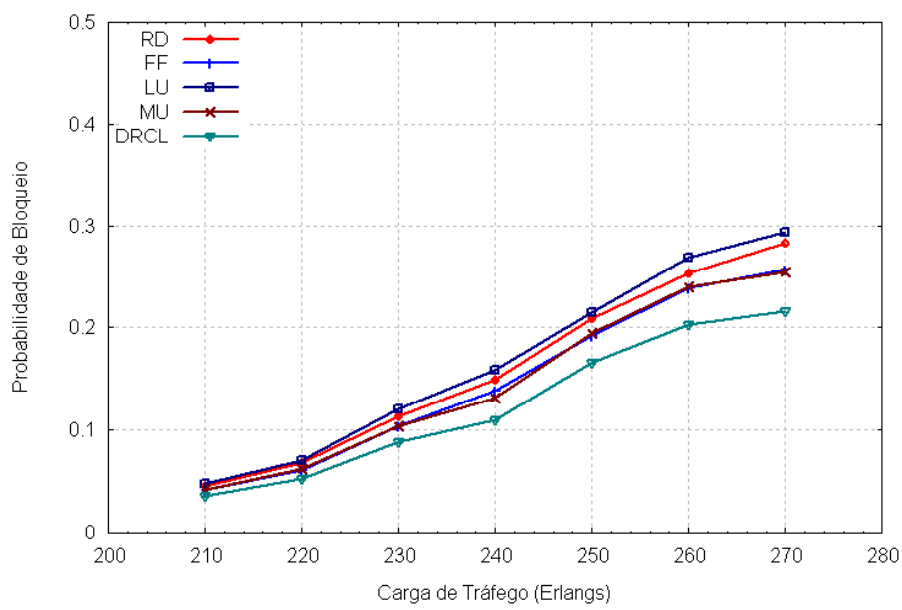

(a)

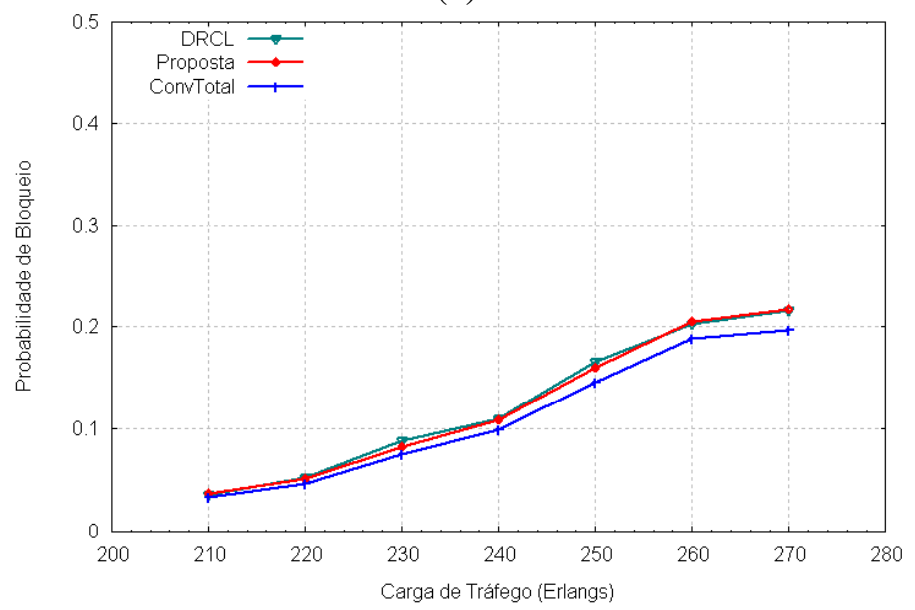

(b)

Figura 6.15 - Topologia NSFNET com 40 comprimentos de onda sem problema de Traps. 
Nota-se também que com o aumento do número de comprimentos de onda disponíveis em cada enlace (aumento de 28\%), o desempenho dos algoritmos DRCL e do proposto se aproximam da situação ótima, promovendo uma diferença máxima de aproximadamente $10 \%$ no desempenho. Desta forma, conclui-se que é mais vantajoso aumentar o número de comprimentos de onda de uma rede, com um custo menor, com o objetivo de melhorar o desempenho ao invés de usar conversores de comprimento de onda que acarretam um custo mais elevado e ainda não apresentam uma tecnologia competitiva.

Nos cenários que apresentam o problema de trap a diferença entre os resultados de desempenho passa a ser maior se os algoritmos propostos pela literatura forem comparados com o algoritmo proposto. A Figura 6.16(a) mostra que as heurísticas continuam apresentando resultados semelhantes, porém com um desempenho inferior em até $38 \%$ se comparado ao mesmo cenário sem o problema de trap.

O algoritmo proposto (Figura 6.16(b)) apresenta desempenho muito superior ao do DRCL, e bem próximo da situação ideal, pois o algoritmo proposto utiliza o conceito SRLG que reconhece o problema de traps fazendo com que o algoritmo não diminua o seu desempenho. Os algoritmos propostos pela literatura não suportam o problema de traps promovendo assim perdas no desempenho. 


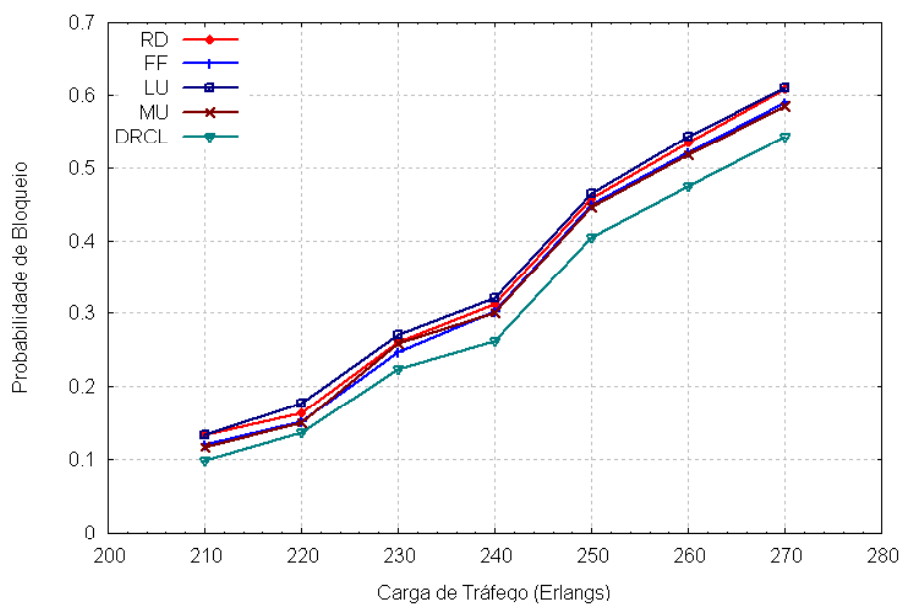

(a)

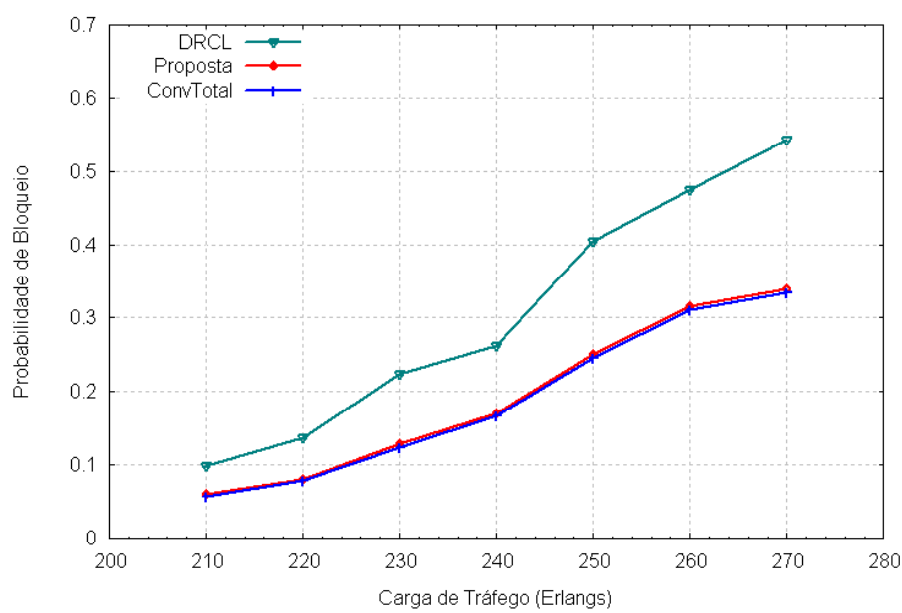

(b)

Figura 6.16 - Topologia NSFNET com 32 comprimentos de onda com problema de Traps.

A Figura 6.17(b) confirma a explanação anterior sobre o desempenho do algoritmo proposto e a proximidade com a situação ideal, pois considera o problema de traps e o número de comprimentos de onda disponível em cada enlace é 40. Assim, pode-se concluir também que quanto maior o número de comprimentos de onda disponíveis menor a probabilidade de bloqueio. 


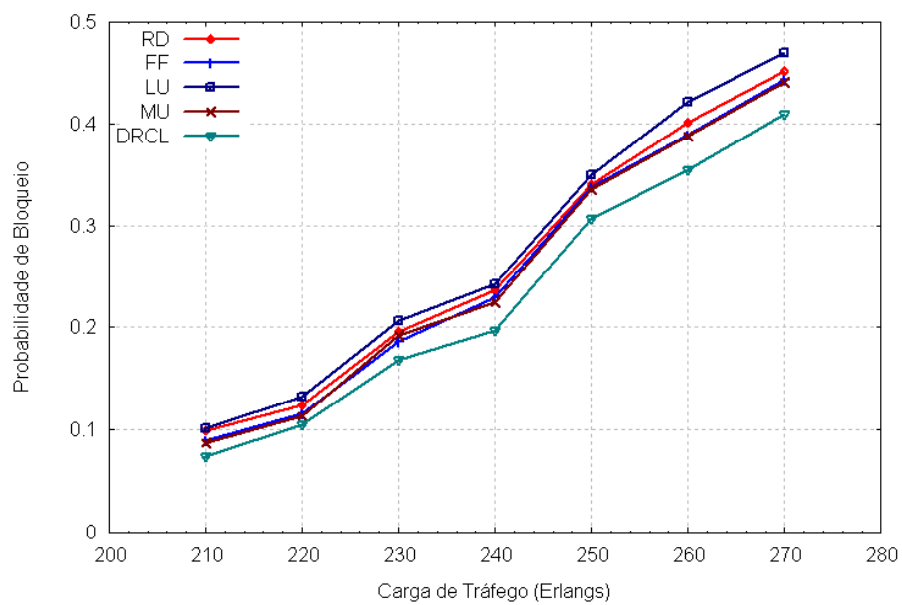

(a)

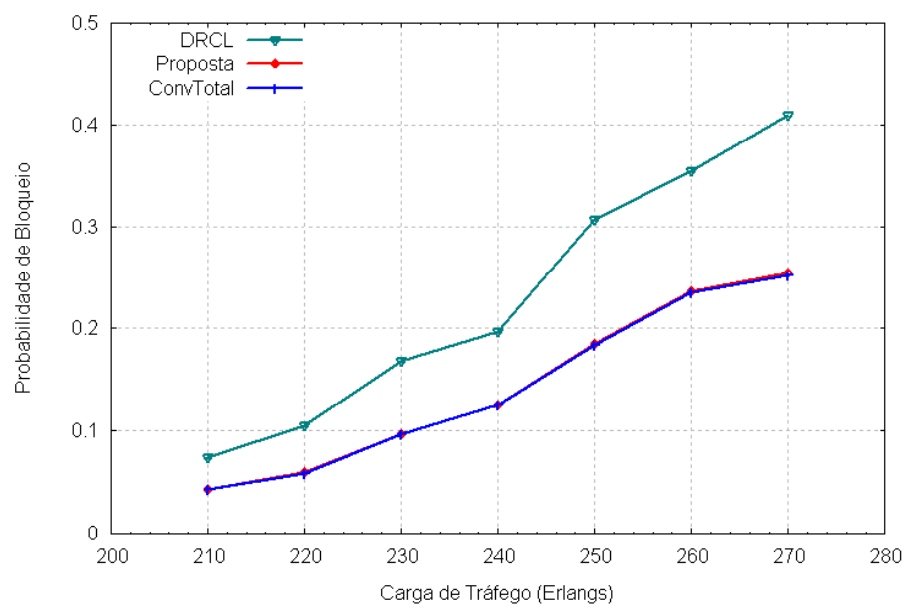

(b)

Figura 6.17 - Topologia NSFNET com 40 comprimentos de onda com problema de Traps.

\subsubsection{Topologia Mesh}

Apresenta-se abaixo as descrições específicas da topologia Mesh 5-5 (Figura 6.18):

- Número de nós: 25

- Número de enlaces: 50

- Número de comprimentos de onda por enlace (W): 32 e 40

- Número de fibras (M): 1

- Número de enlaces com trap: 17 (aproximadamente 35\% do número total de enlaces) 


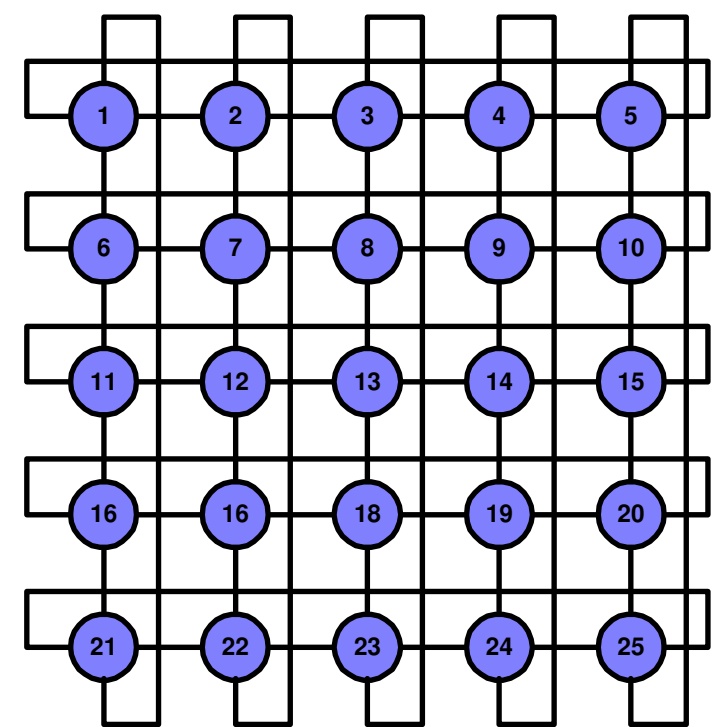

Figura 6.18 - Topologia Mesh com 25 nós.

A topologia Mesh com 32 comprimentos de onda disponíveis em cada enlace (Figura 6.19) confirma as conclusões anteriores a respeitos dos algoritmos RD, FF, LU, MU e DRCL. Todos estes algoritmos propostos pela literatura apresentam diferentes resultados de probabilidade de bloqueio de conexão, mas essa diferença não é significativa (Figura 6.19(a)).

O desempenho apresentado pela topologia Mesh com 32 comprimentos de onda (Figura 6.19) é aproximadamente 5-10\% superior ao desempenho apresentado pela topologia NSFNET com 32 comprimentos de onda em cada enlace. Isso se deve ao fato da probabilidade de bloqueio de conexão ser menor para redes que apresentam uma distribuição uniforme para o comprimento dos enlaces, o que acontece com a topologia Mesh e não acontece com a topologia NSFNET, como mostra as Figuras 6.18 e 6.13, respectivamente. 


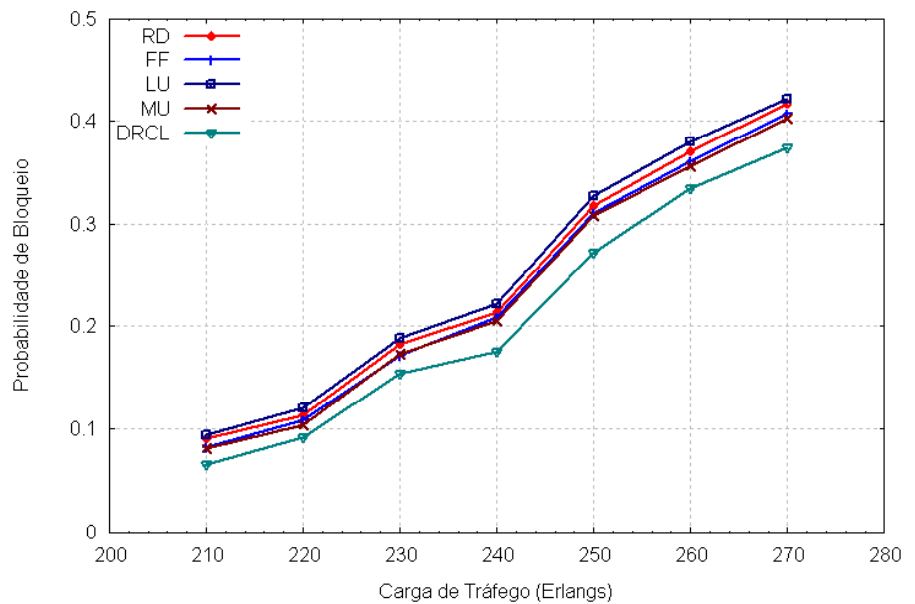

(a)

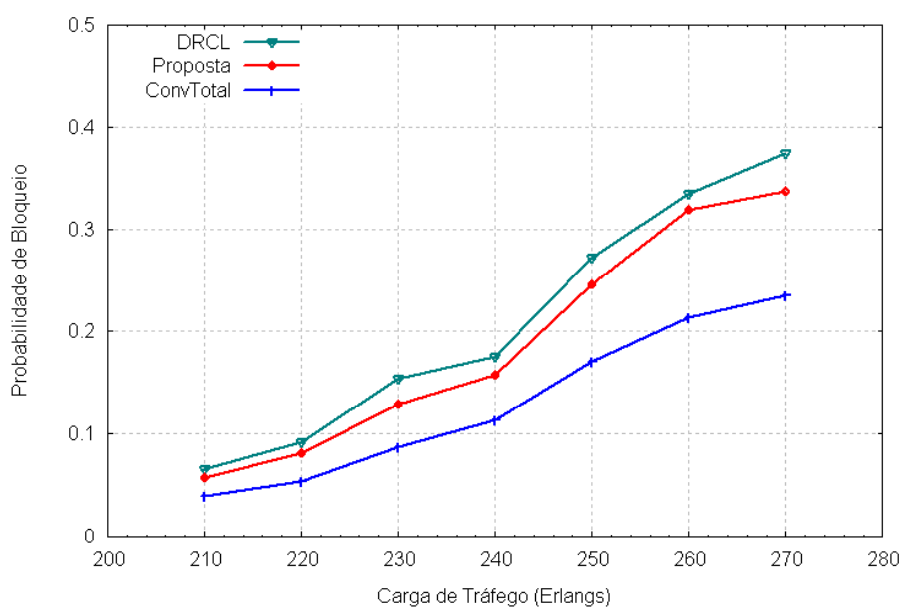

(b)

Figura 6.19 - Topologia Mesh com 32 comprimentos de onda sem problema de Traps.

A figura 6.19(b) apresenta o desempenho do algoritmo proposto, DRCL e da situação ideal (rede com conversão total de comprimento de onda). Nota-se que a probabilidade de bloqueio de conexão da proposta está próxima da situação ideal apresentando valores aproximadamente 45\% maiores quando a carga de tráfego está acima de 240 Erlangs. O resultado da probabilidade de bloqueio de conexão do algoritmo proposto é inferior ao resultado apresentado pelo algoritmo DRCL em aproximadamente 5-10\%. 
Com o aumento no número de comprimentos de onda disponíveis em cada enlace na topologia Mesh, houve um aumento no desempenho da rede, como pode ser observado nas Figuras 6.19 e 6.20. Com o aumento do número de comprimentos de onda em $28 \%$, houve uma melhora no desempenho de aproximadamente $30 \%$, o que é bastante considerável. Como nas demais topologias, com o aumento no número de comprimentos de onda, tanto o algoritmo DRCL como algoritmo proposto se aproximam bastante dos resultados apresentados pela situação ideal, o que garante, mais uma vez, que o aumento no número de comprimentos de onda é mais vantajoso nas redes GMPLS-WDM do que a adoção de conversores de comprimento de onda.

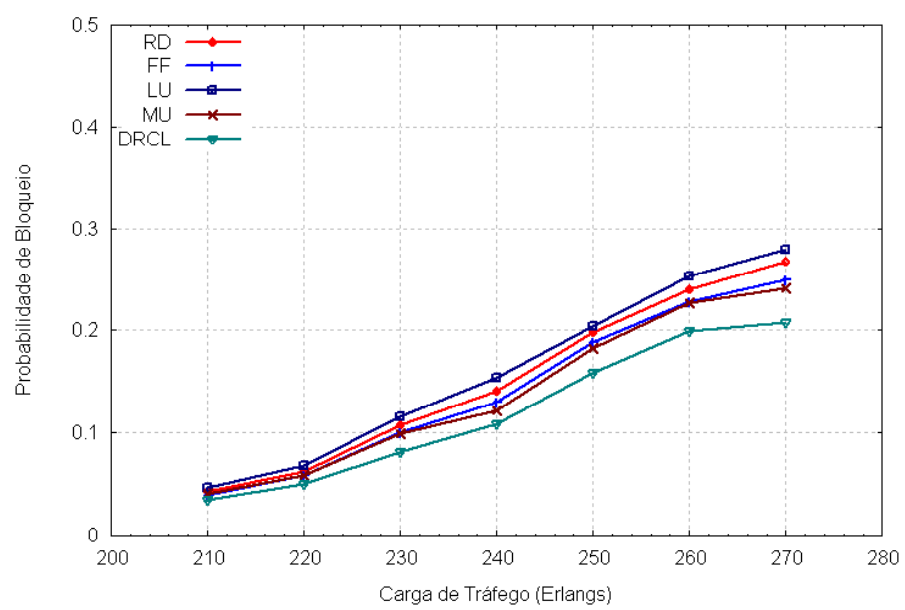

(a)

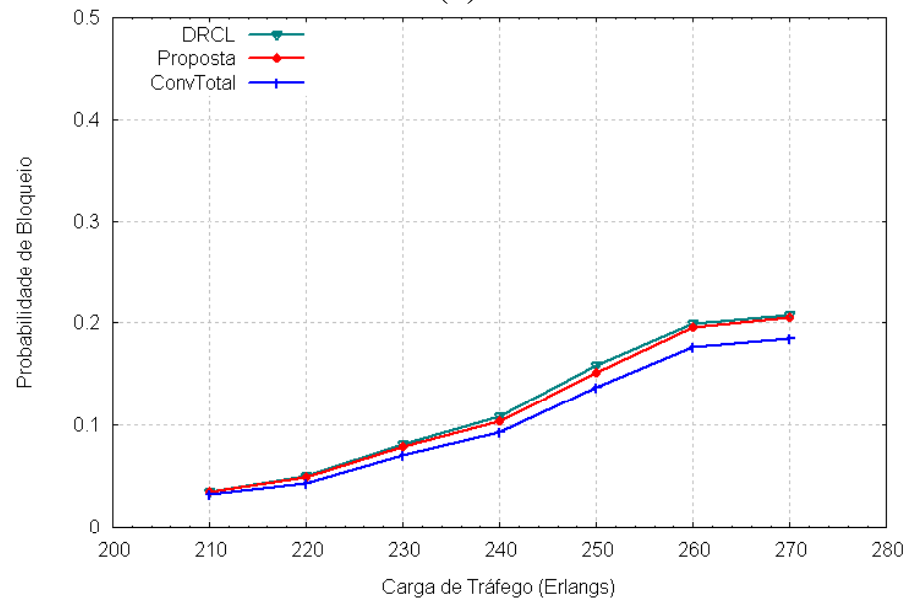

(b)

Figura 6.20 - Topologia Mesh com 40 comprimentos de onda sem problema de Traps. 
De acordo com as Figuras 6.21 e 6.19, ou seja, comparando os cenários de topologia Mesh com 32 comprimentos de onda disponíveis em cada enlace com e sem o problema de traps, verifica-se que os algoritmos de atribuição de comprimento de onda possuem desempenho menor, de aproximadamente $35 \%$, quando são aplicados no cenário com problema de traps. Como mencionado anteriormente, isso se deve ao fato dos algoritmos propostos pela literatura não considerarem o problema de traps no protocolo de sinalização.

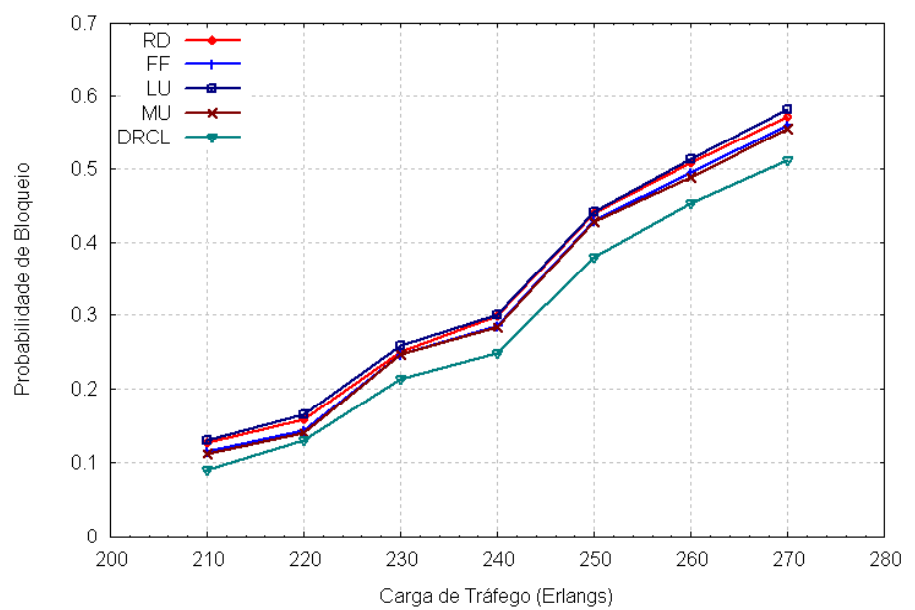

(a)

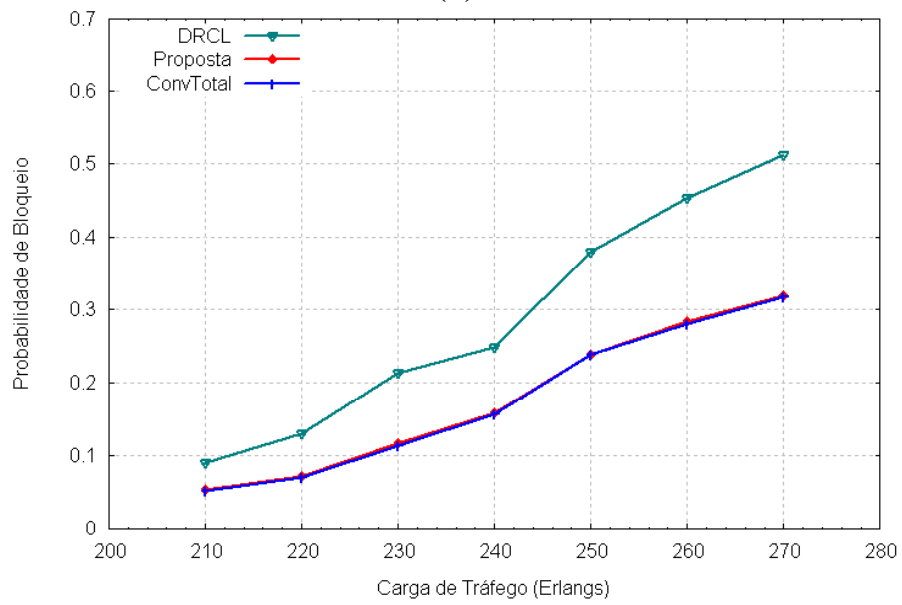

(b)

Figura 6.21 - Topologia Mesh com 32 comprimentos de onda com problema de Traps.

Por outro lado, na topologia Mesh com problema de traps, o algoritmo proposto possui um desempenho próximo do ideal como mostra a Figura 6.21(b). A diferença entre os resultados 
é insignificante, podendo atingir no máximo 4\%. Como o algoritmo DRCL não consegue lidar com o problema de traps, os resultados de probabilidade de bloqueio de conexão apresentados por este algoritmo atingem valores $60 \%$ superiores se comparados aos resultados do algoritmo proposto, principalmente quando a carga de tráfego é maior (acima de 240 Erlangs).

De acordo com a Figura 6.22(a), Topologia Mesh com 40 comprimentos de onda, nota-se que os algoritmos de atribuição de comprimento de onda apresentam um desempenho superior quando os resultados são comparados com os valores de probabilidade de bloqueio no mesmo cenário com 32 comprimentos de onda disponíveis. A melhoria no desempenho foi mantida, pois com o aumento no número de comprimentos de onda em aproximadamente $28 \%$, houve um aumento no desempenho de todos os algoritmos propostos pela literatura em aproximadamente $33 \%$.

Confrontando os cenários, topologia Mesh com 40 comprimentos de onda e problema de traps e topologia NSFNET com 40 comprimentos de onda e problema de traps, verifica-se que na topologia Mesh os resultados apresentam valores aproximadamente 5\% menores se comparados aos resultados de probabilidade de bloqueio de conexão apresentados na topologia NSFNET. Como mencionado anteriormente, isso se deve ao fato da topologia Mesh apresentar uma distribuição mais uniforme do comprimento de enlaces do que a topologia NSFNET. 


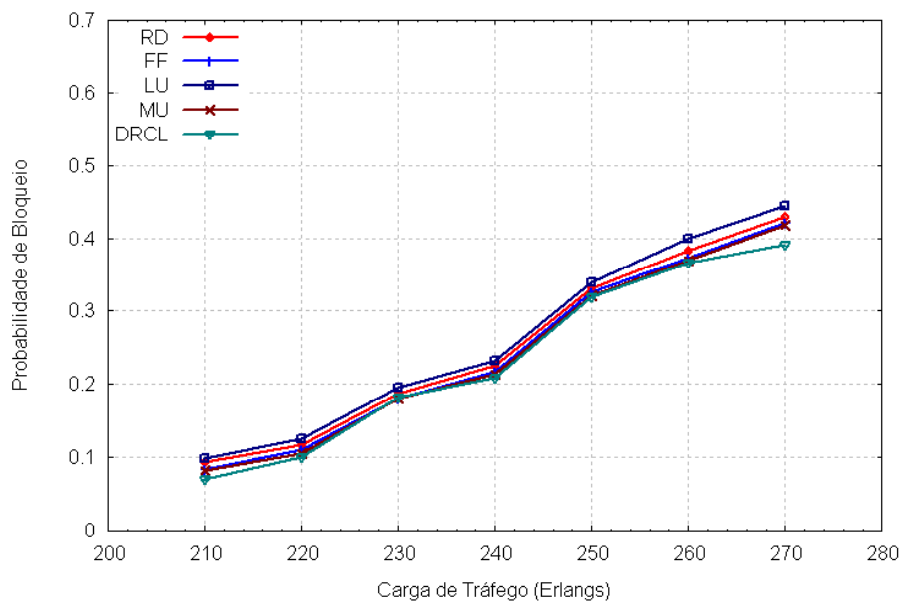

(a)

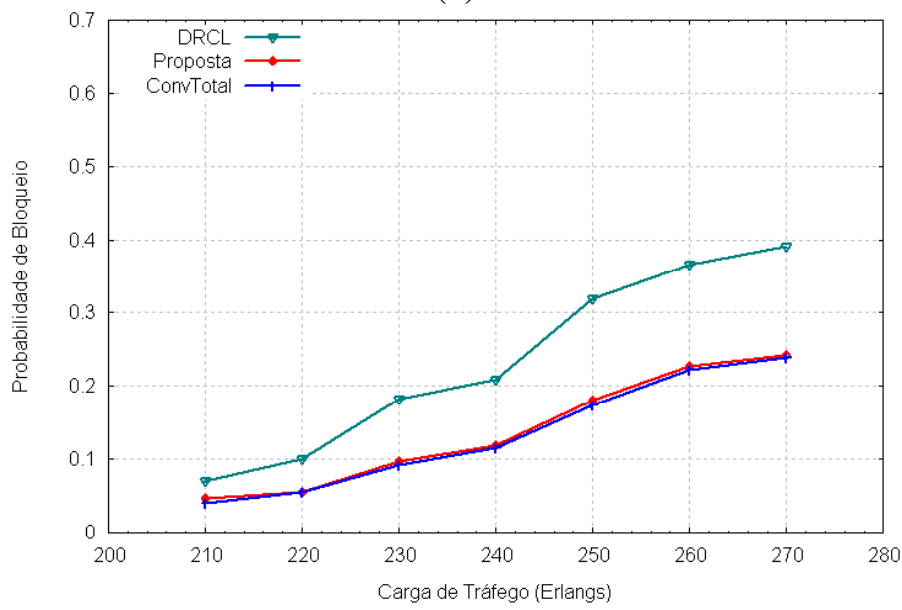

(b)

Figura 6.22 - Topologia Mesh com 40 comprimentos de onda com problema de Traps.

\subsubsection{Topologia EON}

Apresenta-se abaixo as descrições específicas da topologia EON (European Optical Network) (Figura 6.23):

- Número de nós: 19

- Número de enlaces: 39

- Número de comprimentos de onda por enlace (W): 32 e 40

- Número de fibras $(\mathrm{M}): 1$

- Número de enlaces com trap: 14 


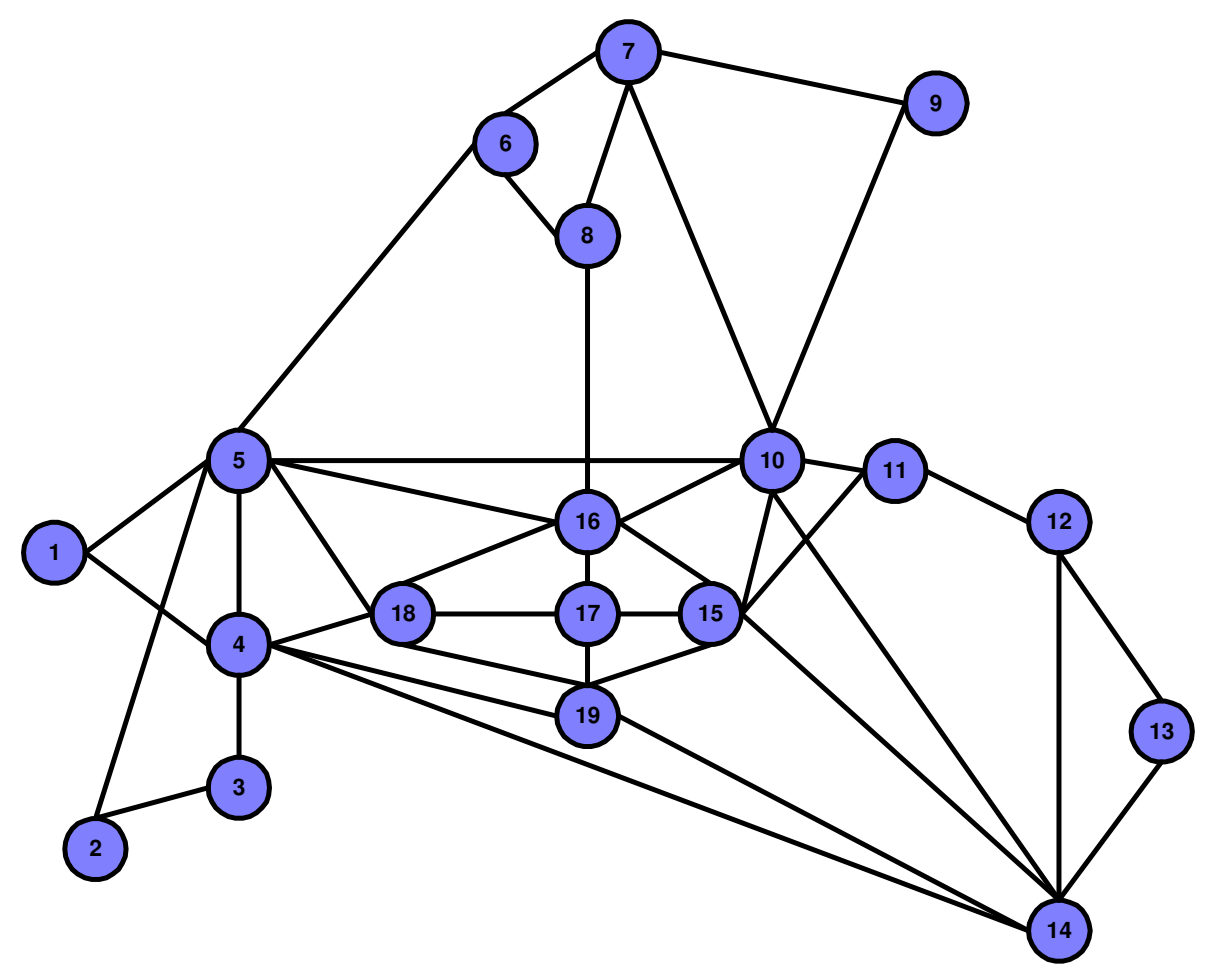

Figura 6.23 - Topologia EON.

Aprovando os resultados obtidos nos cenários anteriores, na topologia EON o algoritmo de atribuição de comprimento de onda DRCL apresenta o melhor desempenho se comparado aos demais algoritmos propostos pela literatura, atingindo resultados aproximadamente $15 \%$ superiores se comparados aos resultados obtidos pelo algoritmo MU, que possui o segundo melhor desempenho.

A figura 6.24(b) mostra a proximidade entre o desempenho obtido pelo algoritmo proposto e o algoritmo DRCL. Ambos os algoritmos possuem resultados próximos da situação ideal que é quando a rede apresenta conversão total de comprimentos de onda. Mas se distanciam da situação ideal (diferença de aproximadamente 45\%) quando a carga de tráfego é superior a 240 Erlangs. 


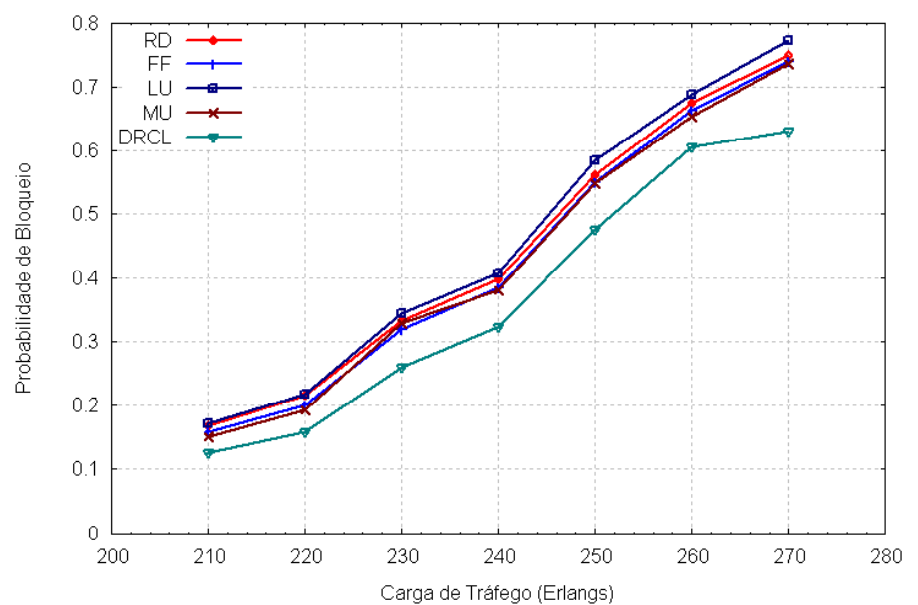

(a)

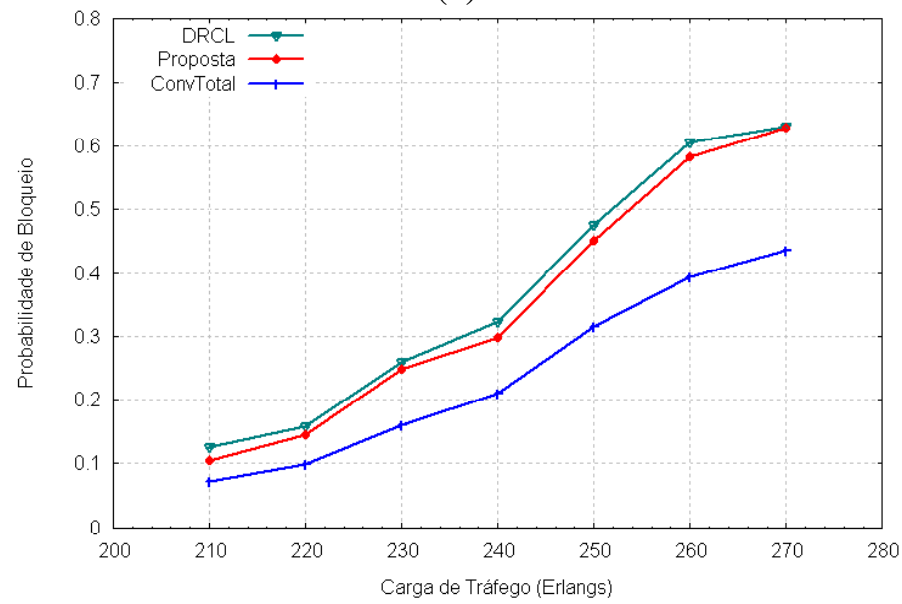

(b)

Figura 6.24 - Topologia EON com 32 comprimentos de onda sem problema de Traps.

Analisando a Figura 6.25, percebe-se que com o aumento de comprimentos de onda disponíveis em cada enlace, o desempenho de todos os algoritmos simulados melhoram, mas as diferenças em porcentagem entre os resultados permanecem praticamente inalterados quando comparados ao cenário anterior (Figura 6.24).

Com o problema de traps na topologia EON, o desempenho diminui em aproximadamente $35 \%$ tanto para a topologia com 32 comprimentos de onda (Figura 6.26) quanto para a topologia com 40 comprimentos de onda (Figura 6.27). 


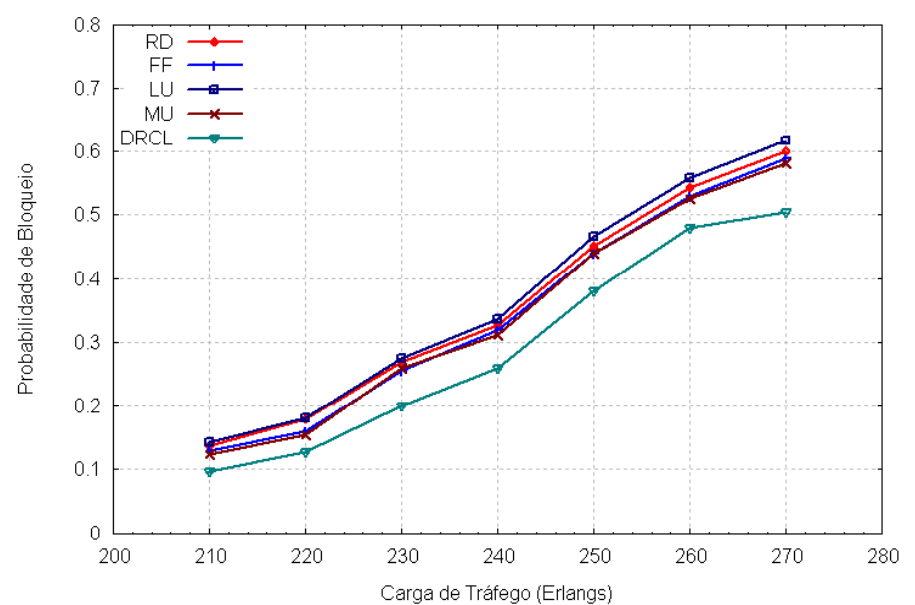

(a)

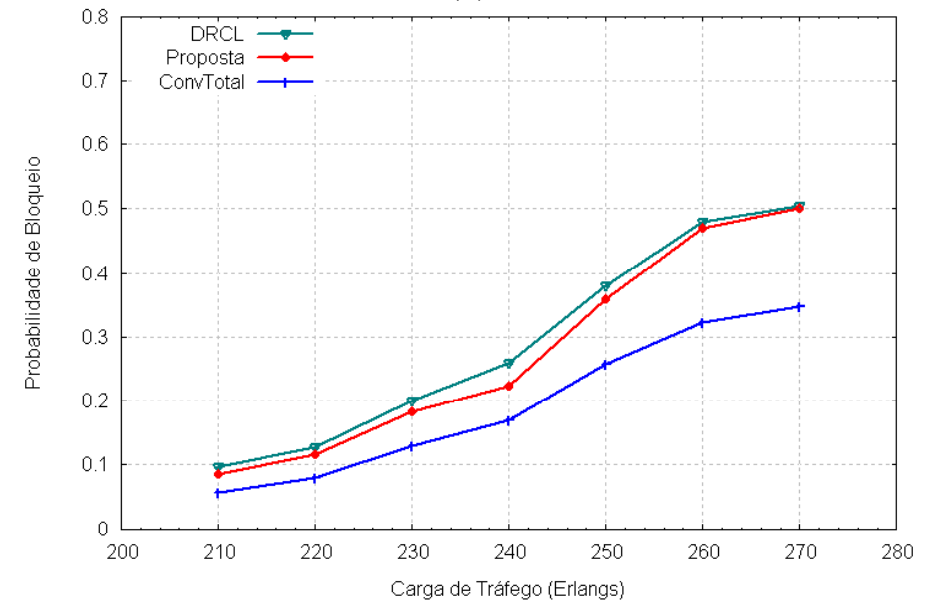

(b)

Figura 6.25 - Topologia EON com 40 comprimentos de onda sem problema de Traps.

Verifica-se que quando o problema de traps é considerado, o algoritmo proposto apresenta um excelente desempenho, próximo do ideal, o que garante que um número razoável de comprimentos de onda (dependendo do tamanho da rede), e um correto algoritmo de atribuição de comprimento de onda, garantem o eficiente uso de recursos da rede durante a sinalização evitando assim o uso de conversores de comprimento de onda, o que tornaria o custo de construção da rede muito maior (Figuras 6.26(b) e 6.27(b)). 


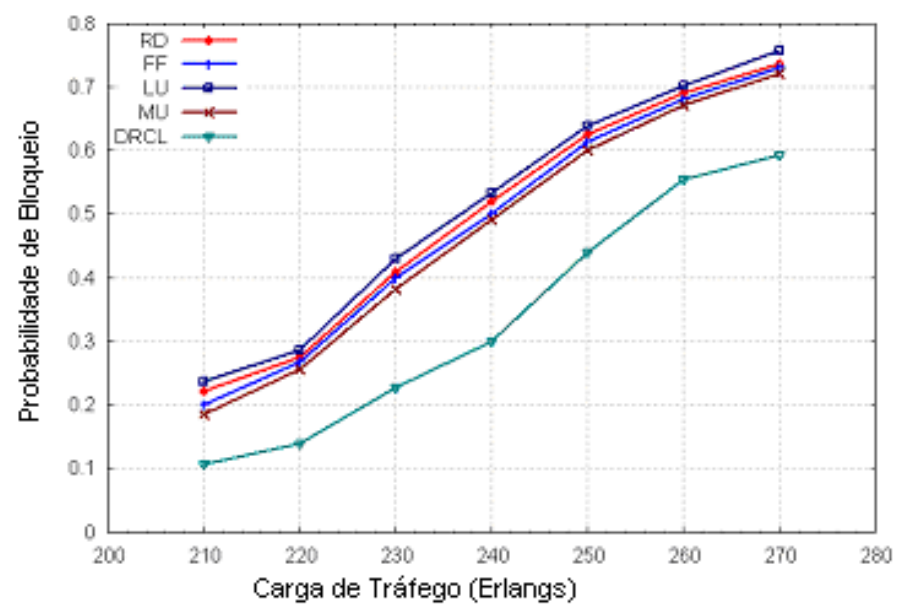

(a)

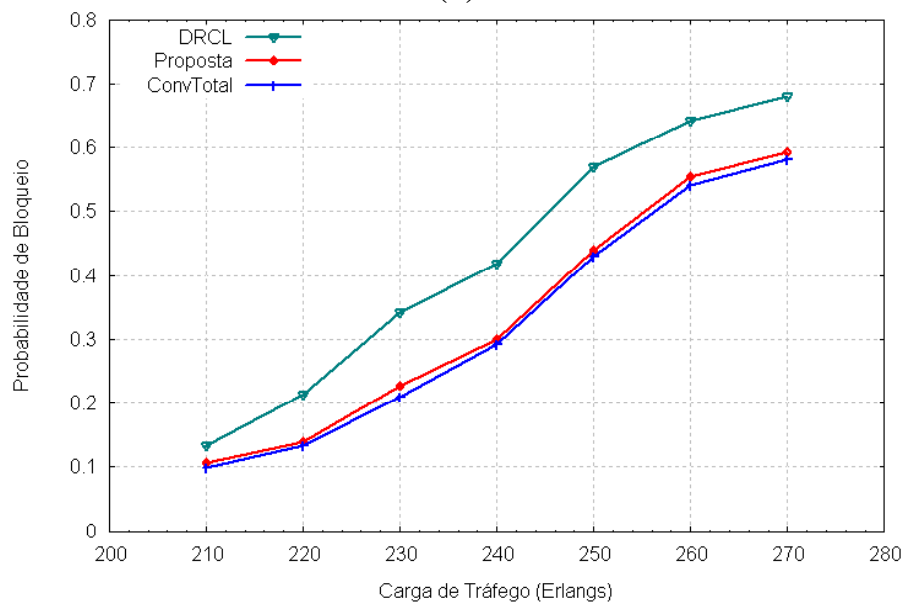

(b)

Figura 6.26 - Topologia EON com 32 comprimentos de onda com problema de Traps.

Comparando as topologias EON e NSFNET quando ambas possuem o mesmo número de comprimentos de onda nos enlaces (32 e 40) e apresentam o problema de traps (Figuras 6.22, 6.23, 6.18 e 6.19), nota-se que o desempenho dos algoritmos na topologia NSFNET é maior em aproximadamente $35 \%$. Isso se deve ao fato, como mencionado em outras comparações, da topologia NSFNET apresentar uma distribuição mais uniforme do comprimento dos enlaces quando comparado a topologia EON. A distribuição uniforme dos comprimentos de onda contribui para uma menor probabilidade de bloqueio dos algoritmos de atribuição de comprimento de onda. 


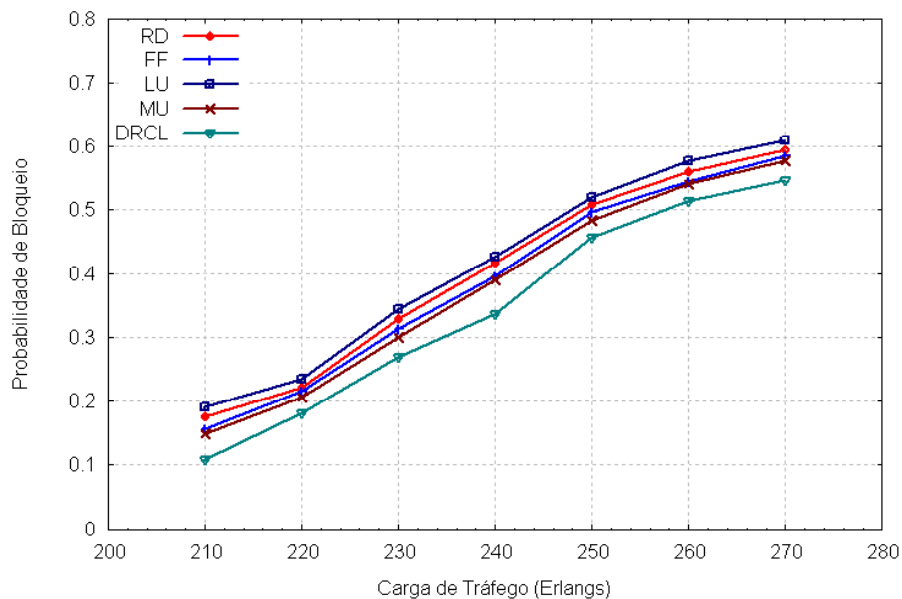

(a)

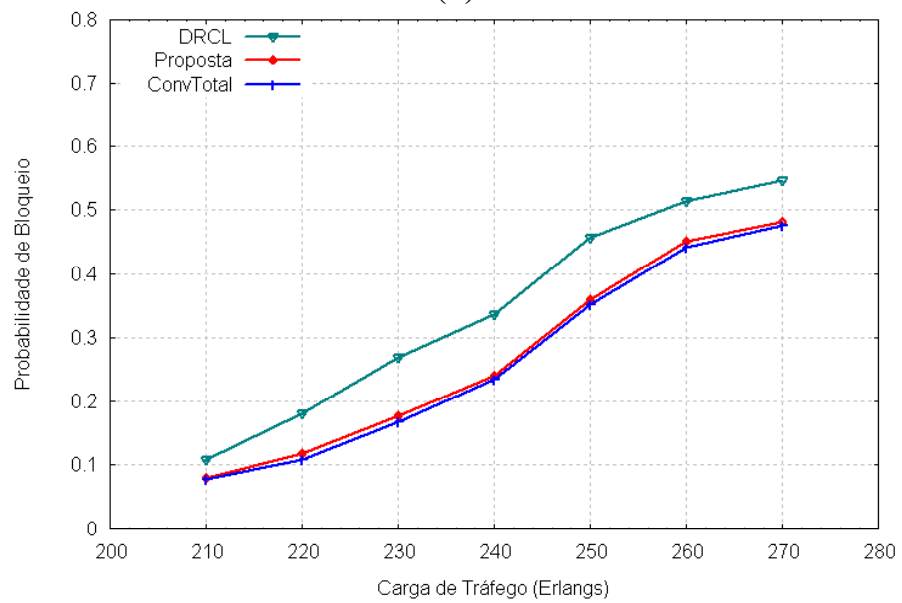

(b)

Figura 6.27 - Topologia EON com 40 comprimentos de onda com problema de Traps.

\subsubsection{Validação do Simulador}

Para garantir a validade de um simulador que avalia o desempenho de um sistema, é necessário fazer a correspondência entre os resultados obtidos através da simulação dos diversos modelos e os dados já existentes sobre sistemas comparáveis ou semelhantes ao sendo modelado. Neste trabalho, a validação dos resultados obtidos por meio dos simuladores utilizados foi realizada através da convergência dos resultados da simulação com os parâmetros e os resultados apresentados em [ZANG, 2000]. 
O parâmetro utilizado na avaliação de desempenho foi a probabilidade de bloqueio de conexão. Logo, é este o parâmetro utilizado na validação dos resultados.

Independente do número de nós nas redes comparadas, os valores da métrica utilizada devem convergir com os valores apresentados na literatura por serem valores em porcentagem.

\subsection{ESCALABILIDADE}

O termo escalabilidade possui diferentes significados, entre eles:

- Propriedade de um sistema qualquer que lhe confere a capacidade de aumentar seu desempenho quando recursos são acrescentados a esse sistema

- Capacidade do hardware e/ou software das redes de alterar o tamanho de acordo com o número de usuários que acomodam. Na maioria dos casos, refere-se à capacidade de expandir em vez de reduzir

- Capacidade de ser alterado para atender um problema específico

Com base nas definições apresentadas, segue uma análise de escalabilidade do algoritmo de rótulos proposto.

A largura de banda necessária para atender as mensagens de controle (sinalização) no algoritmo proposto apresenta um aumento de aproximadamente $10 \%$ se comparado ao procedimento de conjunto de rótulos utilizado isoladamente. Esse aumento é devido a informação SRLG associada a cada comprimento de onda selecionado durante a atribuição de comprimento de onda (como mencionado na seção 6.2.2). No entanto, a largura de banda requerida pelo algoritmo proposto é similar a quantidade requerida pelo algoritmo DRCL. 
Logo, pode-se concluir que o algoritmo proposto não gera um overhead significativo, pois mensagens adicionais não são geradas e o mesmo não interfere no tempo de encaminhamento dos dados bem como na quantidade de dados encaminhados. Esse pequeno overhead gerado pode ser justificado também pelo bom desempenho do algoritmo proposto, como observado na avaliação dos resultados (seção 6.3).

A quantidade de informação sendo encaminhada ao longo da rede para atualização e manutenção dos comprimentos de onda e SRLG-IDs aumenta como também a quantidade de informação armazenada em cada nó intermediário e de borda. Logo, os nós devem ter uma capacidade de armazenamento maior que é tolerável, visto que não interfere no tempo de processamento das mensagens em cada nó (conclusão confirmada através dos resultados da Figura 6.28 onde o algoritmo proposto apresenta desempenho semelhante ou melhor que o desempenho do algoritmo DRCL em termos de tempo de atribuição de comprimento de onda). Essa informação armazenada é proporcional a quantidade de SRLG-IDs utilizados por um nó específico, na ordem de \#SRLG_ID*W (número de SRLG-IDs vezes o número de comprimentos de onda disponível para uso), pois ele armazena apenas as informações referentes as suas interfaces.

Em termos de número de comprimentos de onda, pode-se afirmar que o algoritmo apresenta excelente escalabilidade. O desempenho do algoritmo proposto é melhor com o aumento do número de comprimentos de onda disponíveis em cada enlace, pois o nó de destino terá uma flexibilidade maior para atribuir diferentes comprimentos de onda para tráfegos que utilizam enlaces em comum (o número de opções de possíveis rótulos será maior, o que diminui a probabilidade de bloqueio de conexão). 
O tempo de estabelecimento de uma conexão é definido como o tempo requerido para que a conexão seja estabelecida, o que inclui roteamento e atribuição de comprimento de onda para o pedido de conexão específico. Considerando apenas o tempo para atribuição de comprimento de onda, os fatores que podem afetar este são: atraso de propagação da origem ao destino e vice-versa, atraso no processamento de mensagem, tempo para transmitir ou comutar uma mensagem (considerado como zero) e o tempo de configuração do comutador óptico (caso necessário). Com o aumento da carga de tráfego na rede, ou seja, com o aumento do número de pedidos de conexão em um curto período de tempo, há um aumento no tempo de atribuição de comprimento para uma rota específica que atende um pedido de conexão que varia de 5-9\% do tempo gasto quando a carga de tráfego é menor. Esse aumento é devido ao número maior de mensagens em cada nó esperando por processamento e consulta à tabela SRLG- $\lambda$. No entanto, se a carga de tráfego for muito alta, ultrapassando os limites que a rede suporta, o tempo de atribuição de comprimento diminui, pois as conexões passam a ser bloqueadas devido a não existência de recursos disponíveis.

Como mostra a Figura 6.28 e 6.29, o algoritmo proposto apresenta o tempo de atribuição de comprimento de onda similar ao algoritmo DRCL. Note que com o aumento da carga de tráfego, o tempo de atribuição de comprimento de onda sobe de aproxidamente 1,23ms para 1,32ms (aumento de 6,5\%) para a topologia com 6 nós e há um aumento de 1,34 para 1,39 (aumento de aproximadamente $8,5 \%$ ) para a topologia NSFNET. 


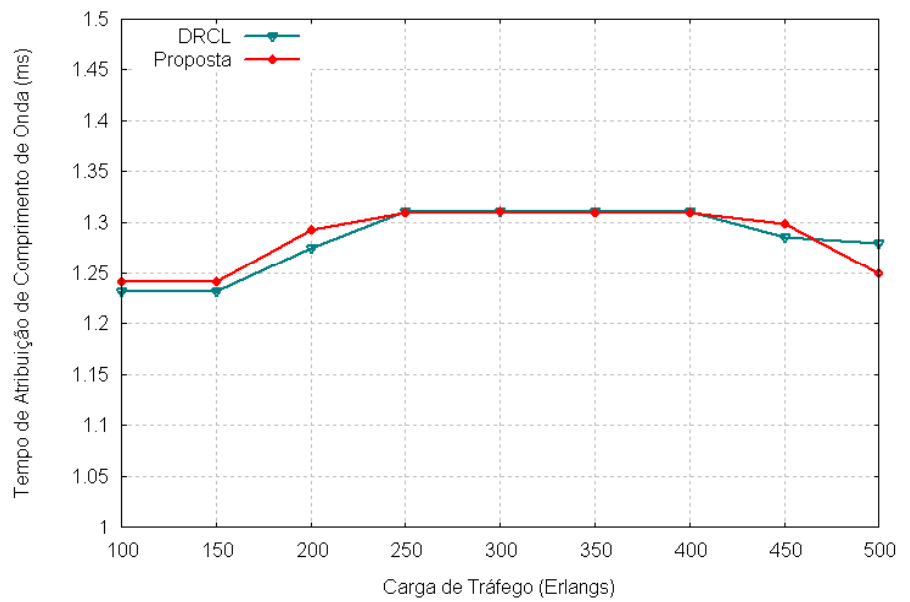

Figura 6.28 - Tempo de Atribuição de Comprimento de Onda na Topologia de 6 nós.

Observe também que com o aumento no número de nós da rede há um pequeno aumento no tempo de atribuição de comprimento de onda devido principalmente ao aumento no tempo de propagação de mensagens entre a origem e destino e, vice-versa. Esta diferença no tempo de atribuição de comprimento de onda para topologias com menor e maior número de nós pode ser verificada nas Figuras 6.28 e 6.29, topologia de 6 nós e topologia de 14 nós, respectivamente.

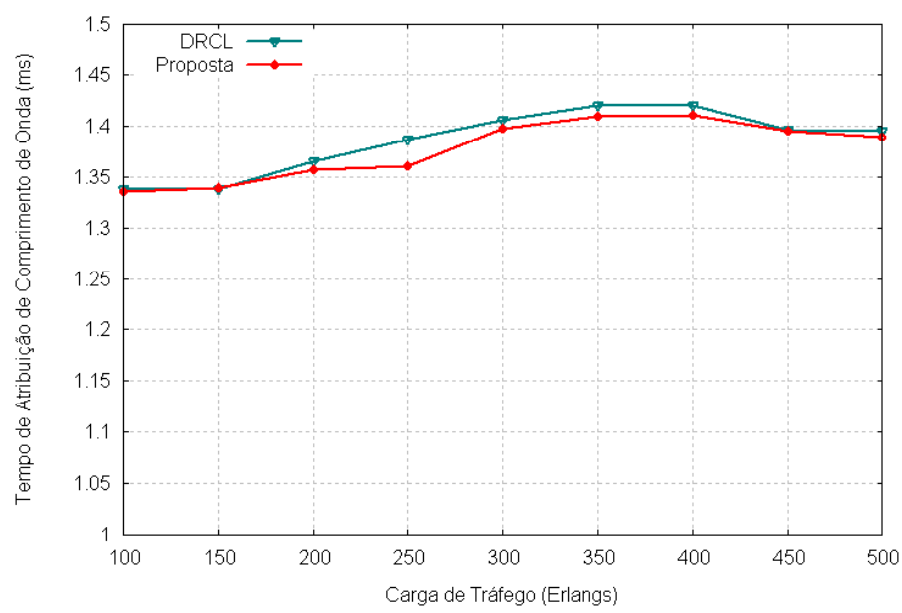

Figura 6.29 - Tempo de Atribuição de Comprimento de Onda na Topologia NSFNET. 


\subsection{CONCLUSÃO}

Com o objetivo de diminuir a probabilidade de bloqueio de conexão foi proposto um algoritmo de atribuição de comprimento de onda que utiliza o conceito de conjunto de rótulos, já implementado pelo GMPLS, e o conceito SRLG, antes usado apenas para o estabelecimento de caminhos de proteção disjuntos.

O número SRLG ou SRLG-ID é estabelecido para cada enlace na rede indicando a entidade de risco a qual o enlace pertence. Um caminho óptico é representado por um conjunto de SRLG-IDs, denominado SRLG_caminho, que contém as entidades de risco de todos os enlaces que compõem o caminho. Caminhos ópticos diferentes que possuem pelo menos um SRLG-ID em comum, certamente, compartilham uma entidade de risco, ou seja, disputam o mesmo recurso, o mesmo enlace e por essa razão deve-se atribuir diferentes comprimentos de onda para esses caminhos ópticos.

Isso se deve ao fato da rede considerada neste estudo ser uma rede GMPLS-WDM parcialmente óptica e sem conversão de comprimento de onda. Logo não há conversores nos nós intermediários da rede e a propriedade de restrição de continuidade de comprimento de onda deve ser considerada e respeitada.

O algoritmo proposto, SRLG mais conjunto de rótulos, funciona basicamente da mesma forma como o objeto conjunto de rótulos já usado em um ambiente GMPLS. No entanto, o conceito de conjunto de rótulos quando usado isoladamente não consegue atender o problema de traps na alocação de recursos. Por outro lado, SRLG mais conjunto de rótulos, juntos, 
conseguem produzir resultados corretos de possíveis comprimentos de ondas a serem escolhidos para atribuição, mesmo quando o problema de trap existe.

Portanto, em ambientes na presença de traps, o algoritmo de atribuição de comprimento de onda proposto consegue diminuir a probabilidade de bloqueio de conexão das redes GMPLSWDM com restrição de continuidade de comprimento de onda.

Foi realizada a análise lógica da porção do protocolo de sinalização responsável pela atribuição de comprimentos de onda com o uso de conjunto de rótulos e SRLG e verificou-se que o mesmo é livre de qualquer erro de progresso. Apenas apresenta um aumento na quantidade de informação de controle sendo enviada na rede.

Para verificar a eficiência do algoritmo proposto, simulações com diferentes cenários foram realizadas utilizando os simuladores NS e Matlab.

De acordo com os resultados apresentados, o algoritmo proposto apresenta resultados próximos do algoritmo DRCL em cenários sem problema de traps, sendo que o DRCL é o algoritmo proposto pela literatura com melhor desempenho.

Nos cenários com problema de traps, o algoritmo proposto apresenta um excelente desempenho, pois possui resultados de probabilidade de bloqueio de conexão inferiores ao do algoritmo DRCL e bem próximos da situação ideal, garantindo assim, sua eficiência. Portanto, pode-se assegurar que o uso de um bom algoritmo de atribuição de comprimento de onda é mais vantajoso do que utilizar conversores de comprimento de onda a um custo elevado. 
Através das simulações, pode-se notar que o número de comprimentos de onda disponíveis nos enlaces interfere diretamente no desempenho dos algoritmos de atribuição de comprimento de onda e essa interferência é diretamente proporcional.

O valor da probabilidade de bloqueio de conexão também pode ser alterada de acordo com a distribuição uniforme ou não do comprimento dos enlaces. Quanto mais uniforme for essa distribuição, menor será a probabilidade de bloqueio de conexão.

Assim, através dos resultados obtidos pelas simulações pode-se garantir a eficiência do algoritmo proposto. 


\section{CAPÍTULO VII}

\section{CONCLUSÕES FINAIS}

Para satisfazer, de forma mais eficiente e a um custo menor, o explosivo aumento na demanda de tráfego de voz e de dados, as redes ópticas baseadas em WDM e GMPLS estão sendo desenvolvidas. O desenvolvimento das redes ópticas provê aumento da capacidade de transporte e da escalabilidade da rede e, o desenvolvimento de uma grande quantidade de novas e sofisticadas aplicações.

As redes ópticas podem ser classificadas em opacas ou transparentes. As redes consideradas neste estudo são as redes parcialmente transparentes. Transparentes, pois as funções de roteamento, tal como comutação de comprimentos de onda, são executadas no domínio óptico eliminando os conversores OEO e suas limitações. Parcialmente, pois a sinalização utiliza canais opacos para que informações sejam armazenadas em nós intermediários.

O encaminhamento de tráfego nas redes ópticas pode ser feito com conversão de comprimento de onda e sem conversão de comprimento de onda. Neste estudo, foram utilizados cenários ópticos que não realizam a conversão de comprimento de onda, ou seja, apresentam restrição de continuidade de comprimento de onda, pois os conversores de 
comprimento de onda ainda possuem um alto custo e a tecnologia não está amadurecida e competitiva.

Inicialmente as redes ópticas utilizavam o TDM como técnica de multiplexação subutilizando assim, a enorme capacidade de transmissão das fibras ópticas. Com o objetivo de melhorar o aproveitamento da capacidade de transmissão das fibras ópticas, as redes ópticas passaram a usar o WDM que é uma técnica promissora por satisfazer altas demandas de largura de banda.

O GMPLS, com suporte do IETF e OIF, é atualmente considerado como um plano de controle para as redes ópticas e suporta não somente comutação de pacotes (PSC), mas também comutação no domínio do tempo (TSC), comprimento de onda (LSC) e fibra (FSC).

O conjunto de protocolos GMPLS é composto por protocolos de sinalização (RSVP-TE) e de roteamento (OSPF-TE), como também do protocolo de gerenciamento de enlace (LMP). As principais funcionalidades do LMP são: gerenciamento de canal de controle, correlação de propriedade de enlace, gerenciamento de falhas e verificação de conectividade de enlace. Este protocolo apresenta grande importância em um ambiente GMPLS podendo também interferir na atribuição de rótulos (comprimentos de onda) e assim, torna-se necessário realizar a análise lógica do LMP para verificar se o mesmo está livre de erros de progresso. O protocolo de gerenciamento de enlace foi então modelado por uma rede formada por duas máquinas de estado finitas de comunicação (MEFC) e, a análise foi feita utilizando a técnica de alcançabilidade com progresso justo podendo assim verificar se o mesmo está ou não livre de erros tais como deadlocks, recepções não-especificadas, interações não-executadas, livelocks e overflows. 
De acordo com os grafos de alcançabilidade justa gerados pela análise com progresso justo, o protocolo de gerenciamento de enlace utilizado em um ambiente GMPLS está livre, parcialmente, dos erros de progresso. No entanto, para tornar o LMP totalmente livre de erros de progresso e fazer com que este tenha um melhor sucesso na ordem de execução e lógica de execução, alguns pequenos detalhes de especificação foram corrigidos e outros sugeridos. Assim, com base nas definições e especificações de cada procedimento LMP, foi proposta uma ordem de execução destes procedimentos:

(v) Gerenciamento de canal de controle

- Ativação do canal de controle

- Manutenção do canal de controle

(vi) Verificação de conectividade de enlace

- Pelo menos um canal de controle deve estar ativo

- Descobrimento dinâmico das associações entre Interface_IDs local e remoto de um mesmo enlace de dados

- Verificação de conectividade de enlaces de dados

- Descobrimento dinâmico de associações de enlace TE

(vii) Correlação de Propriedade de enlace

- Mapeamento de Interface_IDs deve ser um conhecimento já adquirido pelo nó local

- Agregação de múltiplos enlaces de dados a um enlace TE

- Sincronização de propriedades de enlace

(viii) Gerenciamento de Falhas

- Notificação do status de enlaces

- Localização de falhas 
Esta ordem deve ser seguida quando a rede for colocada em funcionamento, pois nesse caso, a maior parte das informações serão geradas e descobertas dinamicamente. No decorrer do funcionamento da rede, essa ordem de execução dos procedimentos LMP não necessariamente precisa ser seguida na maioria dos possíveis cenários.

Verificada a corretude do LMP, o estudo foca o subproblema de atribuição de comprimento de onda do RWA nas redes GMPLS-WDM por ser um dos principais problemas que causam o baixo desempenho destas redes.

O problema RWA é a configuração de caminhos ópticos para um dado conjunto de pedidos de conexões através de roteamento e atribuição de comprimentos de onda para cada uma dessas conexões, dada uma restrição no número de comprimentos de onda disponíveis. O objetivo, portanto, é selecionar a melhor combinação de rotas e comprimentos de onda para cada conexão de forma a otimizar o atendimento das demandas dentro da rede, maximizando o número de conexões estabelecidas.

O RWA pode ser classificado em estático e dinâmico. Neste estudo o RWA dinâmico foi considerado por se aproximar mais da realidade onde um caminho óptico é estabelecido à medida que as requisições de conexões chegam no nó de acesso, devendo cada qual ser atendida no instante da solicitação, sem esperar que futuras requisições se tornem conhecidas.

O problema RWA pode ser dividido em subproblema de roteamento e subproblema de atribuição de comprimento de onda (considerado neste estudo). No subproblema de atribuição de comprimento de onda, deve-se alocar um comprimento de onda para cada caminho óptico de tal forma que dois caminhos ópticos não compartilhem um mesmo comprimento de onda 
em uma mesma fibra e considerando o cenário utilizado que apresenta restrição de continuidade de comprimento de onda. A atribuição de comprimento de onda deve ser feita de forma a maximizar o número de conexões possíveis de serem estabelecidas minimizando assim a probabilidade de bloqueio de novas conexões. Os principais algoritmos de atribuição de comprimento de onda propostos pela literatura são: First-Fit (FF), Random Wavelength Assignment (RD), Most-Used (MU), Least-Used (LU), Max-Sum (MS), Relative Capacity Loss (RCL) e Distributed Relative Capacity Loss (DRCL).

Com o objetivo de melhorar o desempenho das redes GMPLS-WDM com restrição de continuidade de comprimento de onda, diminuindo a probabilidade de bloqueio de conexão, propõe-se um algoritmo de atribuição de rótulos (comprimentos de onda) que utiliza os conceitos conjunto de rótulos e SRLG já implementados pelo GMPLS.

A idéia para alocação de comprimentos de onda é que para conexões cujos caminhos ópticos compartilham pelo menos um enlace em comum na rede, deve-se atribuir diferentes comprimentos de onda para cada conexão, pois a propriedade de restrição de continuidade de comprimento de onda é considerada, como mencionado anteriormente. Esta é a mesma idéia proposta pelo conceito SRLG para o estabelecimento de caminhos de proteção e que agora é usado para alocação de comprimento de onda, como proposta deste estudo. Com base na informação SRLG do caminho a ser estabelecido e dos caminhos já existentes é possível saber se estes compartilham ou não alguma entidade de risco (recurso) e assim decidir qual comprimento de onda deve ser atribuído.

O conceito de conjunto de rótulos quando usado isoladamente não consegue atender o problema de armadilhas na alocação de recursos, denominado de problema de trap. Por outro 
lado, SRLG mais conjunto de rótulos, juntos, conseguem produzir resultados corretos de possíveis comprimentos de ondas a serem escolhidos para atribuição, mesmo quando o problema de trap existe.

A proposta de atribuição de comprimento de onda também foi analisada de acordo com sua corretude, ou seja, se está livre ou não dos erros de progresso e, a técnica de alcançabilidade justa foi usada para esta finalidade. A análise realizada utilizou a porção do protocolo de sinalização responsável pela atribuição de comprimentos de onda com o uso de conjunto de rótulos e SRLG e verificou-se que o mesmo é livre de qualquer erro de progresso.

A análise lógica não é suficiente para comprovar a eficiência ou não eficiência da proposta de atribuição de rótulos. Para esta finalidade, é necessário utilizar um simulador para verificar se a proposta realmente consegue atender ao subproblema de atribuição de comprimento de onda do RWA e comprovar a queda na taxa de probabilidade de bloqueio de conexão para diferentes topologias de rede em um ambiente GMPLS-WDM.

Todas as simulações foram realizadas utilizando o simulador NS com o módulo OWNS e o simulador Matlab com o módulo WDM.

De acordo com os resultados apresentados em todos os cenários simulados, o algoritmo proposto apresenta resultados próximos do algoritmo DRCL em cenários sem problema de traps, sendo que o DRCL é o algoritmo proposto pela literatura com melhor desempenho em qualquer um dos cenários simulados.

O desempenho das heurísticas de atribuição de comprimento de onda apresentadas na literatura é menor nos cenários com problema de trap, pois estas não lidam com problema de 
trap gerando assim um número maior de bloqueio de conexão. O desempenho é aproximadamente $36 \%$ - 55\% menor, dependendo do cenário, se comparado ao desempenho nos cenários que não apresentam o problema de trap. Mas quando a comparação é entre os resultados das heurísticas no mesmo cenário em termos de diferença de desempenhos entre elas, o desempenho é semelhante nos cenários com e sem problema de trap. Os algoritmos MU e FF possuem resultados praticamente idênticos. O algoritmo RD apresenta desempenho inferior se comparado ao algoritmo FF. LU é o algoritmo com maior probabilidade de bloqueio de conexão. No entanto, as diferenças nos resultados apresentados não são significativas. Note que os algoritmos MS e RCL não fazem parte das simulações, pois só podem ser usados em ambientes com RWA estático e as simulações foram realizadas em ambientes com RWA dinâmico.

Nos cenários com problema de traps, o algoritmo proposto apresenta um excelente desempenho, principalmente quando a carga de tráfego é maior, pois o algoritmo proposto reconhece e lida com o problema de trap e conseqüentemente apresenta resultados de probabilidade de bloqueio de conexão inferiores ao do algoritmo DRCL, aproximadamente $35 \%$ - 40\% dependendo do cenário e, bem próximos aos resultados apresentados pela situação ideal (conversão total de comprimento de onda), garantindo assim, sua eficiência.

Portanto, pode-se assegurar que o uso de um bom algoritmo de atribuição de comprimento de onda é mais vantajoso do que utilizar conversores de comprimento de onda a um custo elevado.

Através das simulações, pode-se notar que o número de comprimentos de onda disponíveis nos enlaces interfere diretamente no desempenho dos algoritmos de atribuição de 
comprimento de onda e essa interferência é diretamente proporcional como pode ser visualizado nos gráficos com resultados das simulações na topologia de 6 nós com variação no número de fibras e no número de comprimentos de onda disponíveis em cada enlace, bem como nos demais cenários onde o número de comprimentos de onda foi variado $(32$ e 40 comprimentos de onda).

O valor da probabilidade de bloqueio de conexão também pode ser alterada de acordo com a distribuição uniforme ou não do comprimento dos enlaces. Quanto mais uniforme for essa distribuição, menor será a probabilidade de bloqueio de conexão. Conclusão obtida de acordo com os resultados apresentados pela simulação nas topologias Mesh, NSFNET e EON.

Logo, através dos resultados obtidos pelas simulações pode-se garantir a eficiência do algoritmo proposto nos cenários simulados. O desempenho é verificado através da métrica de probabilidade de bloqueio de conexão, desempenho este próximo do ótimo e demonstrado através das simulações.

Para continuidade deste trabalho sugere-se um estudo de avaliação dos protocolos de roteamento, pois este interfere diretamente no bom desempenho das heurísticas de atribuição de comprimento de onda. Existe também a necessidade de avaliar os diferentes tipos de tráfego de dados uma vez que há possibilidade de cada heurística de atribuição de comprimento de onda apresentar um melhor desempenho de acordo com o tipo de tráfego, ou mesmo propor alterações nestas heurísticas com base nas características de tráfego. 


\section{REFERÊNCIAS BIBLIOGRÁFICAS}

ABOUL-MAGD, O., et. al., A Framework for Generalized Multiprotocol Label Switching (GMPLS), Internet Draft, draft-many-ccamp-gmpls-framework-00.txt, Março 2001, data de expiração Dezembro 2001.

A Transport Network View to LMP, Internet Draft, draft-aboulmagd-ccamptransport-lmp-00.txt, Agosto 2002, data de expiração Fevereiro 2003.

AGILENT Technologies, MPLS and GMPLS: The Key to Network Manageability, Operational Efficiency, and Revenue Generation, 2002.

AKHTAR, S., SOOD, A. K., Performance Evaluation of CSMA/CD Networks Using Markovian Approach, Proceedings International Global Communications Conference, GLOBECOM 88, November 1988.

ANSART, J. P., Issues and Tools for Protocol Specification, The advanced Course on Distributed Systems - Methods and Tools for Specification, Springer-Verlag, pp 481-538, 1985.

ASSI, C., SHAMI, A., ALI, M. A., Optical Networking and Real-Time Provisioning: An Integrated Vision for the Next-Generation Internet, IEEE Network, Agosto, 2001.

BABICH, F., DEOTTO, L., Formal Methods for Specification and Analysis of Communication Protocols, IEEE Communications Surveys. Disponível em: Www.comsoc.org/pubs/surveys.

BANERJEE, A., et. al., Generalized Multiprotocol Switching: an Overview of Routing and Management Enhancements, IEEE Communications Magazine, Janeiro 2001.

et. al., Generalized Multiprotocol Label Switching: An Overview of Signaling

Enhancements and Recovery Techniques, IEEE Communications Magazine, Julho 2001. 
BARRY, R. A., SUBRAMANIAN, S., The Max-sum Wavelength Assignment Algorithm for WDM Ring Networks, OFC'97, 1997.

, HUMBLET, P. A., Models of Blocking Probability in all-Optical Networks with and without Wavelength Changers, IEEE J. Select. Areas Commun, vol 14, pp 858-867, Junho, 1996.

BERGER, L., Generalized Multiprotocol Label Switching (GMPLS) Signaling Functional Description, RFC3471, Janeiro 2003.

Generalized Multiprotocol Label Switching (GMPLS) Signaling Resource Reservation Protocol Traffic Engineering (RSVP-TE) Extensions, RFC3473, Janeiro 2003.

BLUMER, T. P., SIDHU, D. P., Mechanical Verification and Automatic Implementation of Communication Protocols, IEEE Trans. On Software Engineering SE-12(8):827-843, Agosto 1986.

BRADFORD, R., PAPADIMITRIOU, D., TAPPAN, D., Link Management Protocol Extensions for Link Discovery Using Loss of Light, Internet Draft, draft-rbradfor-ccamplmp-lol-01.txt, Outubro 2002, data de expiração Abril 2003.

BRAND, D., ZAFIROPULO, P., On Communicating Finite-State Machines, Journal of the Association for Computing Machinery, vol. 30, no. 2, Abril 1983, pp. 323-342.

CHAUdhuri, S., HJAlMTySSON, G., YATES, J., Control of Lightpaths in an Optical Network, Internet Draft, draft-chaudhuri-ip-olxc-control-00.txt, Fevereiro, 2000.

CHLAmTAC, I., GANZ, A., KARMI, G., Purely Optical Networks for Terabit Communication, IEEE INFOCOM'89, páginas 887-896, 1989.

CHOI, J., et. al., Framework for GMPLS Label Encoding, Internet Draft, draft-choi-gmplslabel-framework-00.txt, Outubro 2002, data de expiração Abril 2003. 
CHOI, T. Y., MILLER, R. E., A Decomposition Method for the Analysis and Design of Finite State Protocols, Proceedings Data Communication Symposium, pp 167-176, ACM SIGCOMM, 1983.

MILlER, R. E., Protocol Analysis and Synthesis by Structured Partitions, Elsevier Science Publishers, 1986.

CHOW, C., GOUDA, M. G., LAM, S. S., A Discipline for Constructing Multiphase Communication Protocols, ACM Transm. On Computer Systems 3(4):315-343, Novembro, 1985.

CHU, X., LI, B., CHLAMTAC, I., Wavelength Converter Placement Under Different RWA Algorithms in Wavelength-Routed All-Optical Networks, IEEE Transactions on Communications, vol 51, no4, Abril, 2003.

COMELLAS, J., et. al., Integrated IP/WDM Routing in GMPLS-Based Optical Networks, IEEE Network , Março / Abril 2003.

CONTE, G., et. al., A Traffic Engineering Solution for GMPLS Networks: A Hybrid Aprroach Based on Off-line and On-line Routing Methods, Ericsson Lab Italy and Coritel, 2003.

CUNHA, D. V., Análise Lógica do Protocolo LMP em um ambiente GMPLS, XXI Simpósio Brasileiro de Telecomunicações, Belém - Pará - Brasil, Setembro, 2004.

CUNHA, D. V., Generalized MPLS - An Overview, ConTel2003, 7th International conference on Telecommunications, Croácia, Junho, 2003.

CUNHA, D. V., Proposta e Avaliação de desempenho de uma Política de Mapeamento de Rótulos Orientado a Controle com Características Soft-State em uma Rede Label Switching, dissertação de mestrado, Universidade Federal de Uberlândia, Maio, 2001.

DATA CONNECTION, Portable LMP. Disponível em: www.dataconnection.com/lmp. 
DEO, G., PRINZ, R., Generalized Multiprotocol Label Switching (GMPLS) Multilayer, Seminar on Topics in Communications Engineering, Institute for Communications Engineering, Institute for Communication Networks, Munich University of Technology, Maio 2003.

EVERdingen, M. V., BERnSteIN, G., Link Management Protocol Update, Internet Draft, draft-everdingen-ccamp-lmp-update-00.txt, Junho 2002, data de expiração Dezembro 2002.

GALLAHER, R., Introduction to Multi-Protocol Lambda Switching (MP $\lambda S$ ) and Generalized Multiprotocol Label Switching (GMPLS), Converge Network Digest, Março 2002.

GIRARD, A., Routing and Dimensioning in Circuit Switched Networks, Addison-Wesley, 1990.

GOUDA, M. G., CHANG, C. K., A Technique for Proving Liveness of Communicating Finite State Machines With Examples, ACM 0-89791-143-1/84/008/0038, 1984, pp/ 38 49.

HAN, J. Y., Protocol Validation by Fair Progress State Exploration, Elsevier Science Publishers, 1985.

, KUO, C. K., CHANG, C. K., Proving Liveness for networks of communicating Finite State Machines, ACM Transactions on Programming Languages and Systems, Vol. 8, No. 1, January 1986, pp. 154-182.

, YU, Y. T., Protocol Validation by Maximal Progress State Exploration, IEEE Transactions on Communications, Vol Com 32, No 1, January 1984.

YU, Y. T., Synthesis of Communicating Finite-Sate Machines with Guaranteed Progress, IEEE Transactions on Communications, Vol Com 32, No 7, July 1984. 
HARAI, H., MURATA, M., MIYAHARA, H., Performance of Alternate Routing Methods in All Optical Switching Networks, IEEE INFOCOM'97, vol 2, páginas 516-524, 1997.

HOLZMAnN, G. J., An Improved Protocol Reachability Analysis Technique, Software, Practice and Experience, Vol 18, No 2, pp. 137 - 161, 1988.

Automatic Protocol Validation in Argos: Assertion Proving and Scatter Searching, IEEE Trans. On Software Engineering, SE-13(6):683-696, Junho 1987.

On Limits and Possibilities of Automated Protocol Analysis, Invited paper to 7th IFIP WG6.1 International Conference on Protocols Specification, Testing, and Verification, Zurich, Switzerland, 1987, pp. 137-161.

, Tracing Protocol, AT\&T Technical Journal, 64(10):2413-2433, Dezembro 1985.

HUANG, C. M., HUANG, D. T., A Backward Protocol Verification Method, IEEE TENCON'93, pp 515 - 518, 1993.

IEC, International Engineering Consortium, Generalized Multiprotocol Label Switching (GMPLS). Disponível em: www.iec.org.

IETF, Internet Engineering Task Force, www.ietf.org.

IOVAnnA, P., SABElla, R., STTEMBRE, M., A Traffic Engineering System for Multilayer Networks Based on the GMPLS Paradigm, IEEE Network, Março /Abril 2003.

ITOH, M., ICHIKAWA, H., Protocol Verification Algorithm Using Reduced Reachability Analysis, The Trans on the IECE of Japan E66(2):88-93, Fevereiro, 1983.

JERRAN, N., FARREL, A., MPLS in Optical Networks, Data Connection Limited, Versão 2,2001 .

JUE, J. P., XIAO, G., An Adaptative Lightpath Establishment Scheme for Wavelengthrouted Optical Networks, IEEE ICCCN, 2000. 
KAKUDA, Y., WAKAharA, Y., NORIGOE, M., A New Algorithm for Fast Protocol Validation, Proceedings COMPSAC, pp 228-236, IEEE, 1986.

KAO, L.C., TSAI, Z., Performance analysis of A Generic GMPLS Switching Architecture with Flush Capability, IEEE ICC 2002, New York City, Abril 2002.

KARASAN, E., AYANOGLU, E., Effects of Wavelength Routing and Selection Algorithms on Wavelength Conversion Gain in WDM Optical Networks, IEEE/ACM Transactions on Networks, vol. 6, no. 2, Abril, 1998.

KIM, C. H., YANG, C. W., JEON, H., LEE, J. Y., Improved GMPLS Lightpath Setup in Wavelength-Routed Optical Networks, Joint Conference on Communications and Information, 2003.

KOMPELLA, K., REKHTER, Y., IS-IS Extensions in Support of Generalized MPLS, Internet Draft, draft-ietf-isis-gmpls-extensions-14.txt, Agosto 2002, data de expiração Fevereiro 2003.

, REKHTER, Y., OSPF Extensions in Support of Generalized MPLS, Internet Draft, draft-ietf-ccamp-ospf-gmpls-extensions-08.txt, Agosto 2002, data de expiração Fevereiro 2003.

REKHTER, Y., Routing Extensions in Support of Generalized MPLS, Internet Draft, draft-ietf-ccamp-gmpls-routing-05.txt, Agosto 2002, data de expiração Fevereiro 2003.

KRIEGER, U. R., CLOSTERMANN, B. M., SCZITTNICK, M., Modeling and Analysis of Communication Systems Based on Computational Methods for Markov Chains, IEEE Journal on Selected Areas in Communications, vol 8, no. 9, December 1990.

LAM, S. S., SHANKER, A. U., Protocol Verification via Projections, IEEE Trans. On Software Engineering SE-10(4):325-342, Julho, 1984.

LANDOLSI, T., XUE, Y., GMPLS and its Potential Applications: A Reality Check, WorldCom, Março 2002. 
LANG, J., Link Management Protocol (LMP), RFC4204, draft-ietf-ccamp-lmp-10.txt, Outubtro 2005.

LI, G., YATeS, J., WANG, D., KAlMANEK, C., Control Plane Design for Reliable Optical Networks, AT\&T Labs Research, 2002.

LI, L., SOMANI, A. K., Dynamic Wavelength Routing using Congestion and Neighborhood Information, IEEE/ACM Transactions on Networking, 1999.

LIN, F. J., CHU, P. M., LIU, M. T., Protocol Verification Using Reachability Analysis: The State Space Explosion Problem and Relief Strategies, ACM 0-89791-2454/88/0001/0126, 1998.

LUNDY, G. M., AKYILDIZ, I. F., Specification and analysis of the FDDI MAC Protocol Using Systems of Communicating Machines, 1992.

MILleR, R. E., Analyzing a CSMA/CD Protocol Through a Systems of Communicating Machines Specification, IEEE Transactions on Communications, Vol 41, No 3, March 1993.

, MILLER, R. E., Specification and analysis of a Data Transfer Protocol Using Systems of Communicating Machines, Distributed Computing, Vol 5, p 145-157, 1991.

MAIER, G., PATTAVINA, A., BARBATO, L., CECINI, F., MARTINELLI, M., Routing Algorithms in WDM Networks under Mixed Static and Dynamic Lambda-Traffic, Photonic Network Communications, vol. 8, no. 1, pp. 69-87, Julho, 2004.

MANDAL, S., SAHA, D., BERA, S., RAY, S., MAHANTI, A., An Efficient Technique for Dynamic Lightpath Establishment in Survivable Local/Metro WDM Optical Networks, Proc. $5^{\text {th }}$ International Workshops on Distributed Computing, pp. 350-360, Calcutta, Dezembro, 2003.

MANNIE, E., et. al., Generalized Multiprotocol Label Switching (GMPLS) Architecture, Internet Draft, RFC3945, Outubro, 2004. 
MIYAMURA, T., KURIMOTO, T., AOKI, M., MISAWA, A., An Inter-area SRLGdisjoint Routing Algorithm for Multi-segment Protection in GMPLS Networks, International Conference on Communication and Broadband Networking, Japão, Abril, 2004.

MOURA, J. A. B., et. al., Redes Locais de Computadores - Protocolos de Alto Nível e Avaliação de Desempenho, São Paulo, McGraw-Hill, 1986, 454 páginas.

MPLS FORUM. Disponível em: www.mplsforum.org.

MPLSRC, MPLS Resource Center. Disponível em: www.mplsrc.com.

NAGANO, S., et. al., Timed Reachability Analysis Method for EFSM-based Communication Protocols and its Experimental Evaluation, IEEE 1996.

NAM, Network Animator, http://www.isi.edu/nsnam/nam/

NEGUlESCU, R., BRZOZOWSKI, J. A., Relative Liveness: From Intuition to Automated Verification, 2nd Working Conference on Asynchronous Design Methodologies, England, May 1995.

NS, Network Simulator, http://www.isi.edu/nsnam/ns/

OKI, E., MATSUURA, N., SHIOMOTO, K., YAMANAKA, N., Bidirectional Path Setup Scheme Using on Upstream Label Set in Optical GMPLS Networks, IEICE Trans. Communication, vol. E87-B, no. 6, Junho 2004.

OTCL Toolkit, http://www.isi.edu/nsnam/otcl/

OWNS, Optical WDM Network Simulator, http://dawn.cs.umbc.edu/software/owns.html

OZDEMIR, K., URAL, H., Deadlock Detection in CFSM Models via Simultaneously Executable Sets, International Conference on Computing and Information, 1994. 
PAPADIMITRIOU, D., et. al., Analysis for GMPLS-based Recovery Mechanisms, Internet Draft, draft-design-team-gmpls-recovery-analysis-00.txt, Fevereiro 2002, data de expiração Agosto 2002.

, ROUSSEAU, B., Demystifying GMPLS - A Technical Perspective, Technology White Paper, Alcatel, 2003.

, POPPE, F., JONES, J., VENKATACHALAM, S., Shared Risk Link Groups Inference and Processing, Internet Draft, draft-papadimitriou-ccamp-srlg-processing-02.txt, Junho 2003, data de expiração Dezembro, 2003.

PARK, J. C., MILLER, R. E., A Proof Technique for Liveness Properties of Multifunction Composite Protocols, 6th IEEE International Conference on Network Protocols, Austin Texas, October 1998.

PENG, W., Deadlock Detection in Communicating Finite State Machines by Even Reachability Analysis, Mobile Networks and Applications 2, pp 251-257, 1997.

POLARIS NETWORKS, GMPLS Resorce Center. Disponível em: Www.polarisnetworks.com/gmpls.

RAMAMURTHY, S., MUKHERJEE, B., Fixed Alternate Routing and Wavelength Conversion in Wavelength-routed Optical Networks, IEEE GLOBECOM'98, vol 4, páginas 2295-2302, 1998.

RAMASWAMI, R., SIVARAJAN, K. S., Routing and Wavelength Assignment in AllOptical Networks, IEEE/ACM Transactions on Networks, vol. 3, no. 5, Outubro, 1995.

ROUSKAS, G. N., Routing and Wavelength Assignment in Optical WDM Networks, Wiley Encyclopedia of Telecommunications, John Wiley and Sons, 2001.

ROUSSEAU, B., PAPADIMITRIOU, D., Generalized Multiprotocol Label Switching, Strategy White Paper, Alcatel, 2003. 
RUBIN, J., WEST, C. H., An Improved Protocol Validation Technique, Computer Networks, 6:65-73, 1982.

SABNANI, K., An Algorithmic Technique for Protocol Verification, IEEE Transactions on Communications, Vol 36, No 8, August 1988.

SAHA, D., Coverging Optical and IP: Can GMPLS Take Control?, Communication Systems Design, Fevereiro 2002.

SCHOOT, H., URAL, H., On Improving Reachability Analysis for Verifying Progress of Networks of CFSMs, 18th International Conference on Distributed Computing Systems, Amsterdam, May 1998.

SEBOS, P., YATES, J., RUENSTEIN, D., GRENNBERG, A., Effectiveness of Shared Risk Link cGroup Auto-Discovery in Optical Networks, Optical Fiber Communication Conference (OFC2002), Anaheim, CA, 2002.

SHAMI, A., ASSI, C., HABIB, I., ALI, M. A., Performance Evaluation of Two GMPLSBased Distributed Control and Management Protocols for Dynamic Provisioning in Future IP Networks, Proc. Of IEEE, International Conference on Communications, Abril, 2002.

STRAND, J., CHIU, A. L., TKACH, R., Issues for Routing in the Optical Layer, IEEE Communications Magazine, Fevereiro, 2001.

SUBRAMANIAM, S., BARRY, R. A., Wavelength Assignment in Fixed Routing WDM Networks, IEEE ICC'97, páginas 406-410, 1997.

TACHIBAnA, T., KASAHARA, S., Performance Analysis of Dynamic Lightpath Configuration for WDM Tandem Networks, $10^{\text {th }}$ Internacional Conference on Telecommunication Systems: Modelling and Analysis, pp. 679-688, Califórnia - EUA, Outubro, 2002. 
UM, T. W., et. al., Signaling and Control Procedures Using Generalized MPLS Protocol for IP over an Optical Network, ETRI Journal, Volume 24, Number 2, Abril 2002.

VASSEUR, J.P., Protection / Restoration in GMPLS Networks, Cisco Systems, Abril 2001.

VIGOUREUX, M., et. al., GMPLS Architectural Considerations for (Hybrid) Photonic Networks, Internet Draft, draft-vigoureux-ccamp-gmpls-architecture-hpn-00.txt, Junho 2002, data de expiração Dezembro 2002.

VUONG, S. T., COWAN, D. D., A Decomposition Method for the Validation of Structured Protocols, Proceedings INFOCOM, pp 209-220, IEEE, 1982.

HUI, D. D., COWAN, D. D., Valira - A Tool for Protocol Validation Via Reachability Analysis, Protocol specification, Testing, and Verification, VI, North-Holland, pp 35-41, 1987.

WEST, C. H., Applications and Limitations of Automated Protocol Validation, Protocol Specification, Testing, and Verification, II, North-Holland, 1982, pp 361-371.

Protocol Validation by Random State Exploration, Protocol Specification, Testing, and Verification, VI, North-Holland, pp 233-242, 1987.

WHITE ROCK NETWORKS, GMPLS: A New Way of Optical Networking. Disponível em: www.whiterocknetworks.com. Último acesso: Janeiro, 2002.

WIDJAJA, I., ELWALID, A.,. Study of GMPLS Lightpath Setup over Lambda-Router Networks, IEEE International Conference on Communications, 2002.

YEH, W. J., YOUNG, M., Compositional Reachability Analysis Using Process Algebra, Symposium on Testing, Analysis, 1991. 
YEN, Y., Finding the lengths of all shortest paths in $N$-node nonnegative-distance complete networks using $\left(N^{\wedge} 3\right) / 2$ additions and $N^{\wedge} 3$ comparisons, Journal of the ACM, 19(3):423-424, Julho, 1972.

YUANG, M. C., Survey of Protocol Verification Techniques Based on Finite State Machine Models, CH2547-8/88/0000/0164, IEEE, 1988.

ZANG, H., JUE, J. P., MUKHERJEE, B., A Review of Routing and Wavelength Assignment Approaches for Wavelength-Routed Optical WDM Networks, Optical Networks Magazine, vol. 1, no. 1, pp 47-60, Janeiro, 2000.

ZANG, H., JUE, J. P., SAHASRABUDDHE, L., RAMAMURTHY, R., MUKHERJEE, B., Dynamic Lightpath Establishment in Wavelength-Routed WDM Networks, IEEE Communications Magazine, Setembro, 2001.

ZHANG, X., QIAO, C, Wavelength Assignment for dynamic Traffic in Multi-fiber WDM Networks, IEEE ICCCN, páginas 479-485, 1998.

ZHAO, J. R., BOCHMANN, G. V., Reduced Reachability Analysis of Communication Protocols: A New Approach, Protocol Specification, Testing, and verification, VI, NorthHolland, pp 243-254, 1987.

ZHU, Y., ROUSKAS, G. N., PERROS, H. G., A comparison of allocation policies in Wavelength Routing Networks, Photonic Networks Communication Journal, vol. 2, no. 3, pp 265-293, Agosto, 2000. 